\title{
FACTORS AFFECTING THE CARE OF ACUTELY UNWELL WARD PATIENTS: A MULTIPLE CASE STUDY
}

\author{
BY
}

\section{SARA JANE MICHAELA QUIRKE}

A thesis submitted to the Victoria University of Wellington in fulfillment of the requirements for the degree of Doctor of Philosophy in Nursing

Victoria University of Wellington

2011 


\section{ABSTRACT}

Suboptimal care of acutely unwell ward patients is of growing concern internationally. As empirical study of the problem is underdeveloped this thesis explores factors affecting suboptimal care of acutely unwell ward patients. In order to bring a theoretical understanding to this area, a concept analysis of the term suboptimal care was undertaken. The results of this concept analysis were then used to inform the design and analysis of this multiple case study.

The cases for this study were two general surgical and two general medical wards located in two hospitals in the North Island of New Zealand. Interviews and focus groups with nurses, doctors, and managerial staff were undertaken using a semi structured interview approach informed by the concept analysis. Organisational and ward documentation was also reviewed. Using categorical aggregation and pattern matching, an analytical framework emerged from the data. This framework was then used to conduct within, cross case, and hospital analyses.

Key findings of this research reveal that workload, teamwork, communication, leadership, skills and knowledge deficit, and organisational systems and processes are significant factors affecting care of acutely unwell ward patients and that these factors are not unique to specific contexts. Polarised views about workload were expressed by those who manage organisations and those who deliver care at ward level.

Current approaches to improving care of the acutely unwell ward patient have involved the introduction of service initiatives. However, this study demonstrates that service initiatives alone are insufficient to improve care for acutely unwell ward patients. Recommendations from this study are that strategies should be put in place to support and develop clinical shift leaders and that staffing resources should be reviewed in the context of contemporary acute care settings. These must be informed by the views of organisational managers and ward staff leading to an integrated hospital-wide understanding of factors affecting care of the acutely unwell ward patient.

Key words: Suboptimal care, acutely unwell ward patients, multiple case study 


\section{DEDICATION}

This thesis is dedicated to the memory of my dear friend Stacy Burnard ( $9^{\text {th }}$ May $1967-31^{\text {st }}$ August 2010). Stacy's courage and determination was inspirational. 


\section{ACKNOWLEDGEMENTS}

I have been very fortunate to have the support and guidance of some fantastic people over the last four years. It is a pleasure to thank the many people who made this research possible.

Firstly I would like to thank Dr Rose McEldowney, my initial Supervisor, who started me on this journey and introduced me to the world of qualitative research. More latterly I am deeply indebted to my current supervisors, without whom I would never have completed this work. To Dr Maureen Coombs: thank you for listening, for helping me craft my writing, for making me consider an alternative way of looking at things and most of all for believing in me. Your knowledge and expertise is amazing.

To Professor Rowena Cullen: I thank you for your support, thoughtful guidance and encouragement throughout the last 18 months. I will be eternally grateful for the genuine interest you have shown in this research and your tireless ability to keep me going in the right direction.

I wish to express my sincere appreciation to my Head of School, Professor Jo Walton, for giving me the time and space to finish this thesis. Further thanks are expressed to my colleagues at the Graduate School of Nursing, Midwifery and Health for their patient listening and words of advice.

To all the participants who took part in this study - I was truly moved by your willingness to share your experiences and views with me.

My final words of thanks must be dedicated to my husband Terry, and my children Eleanor, Joseph and Isaac. Without your love, support and encouragement I would never have made it to the end. You have all been incredible. 


\section{TABLE OF CONTENTS}

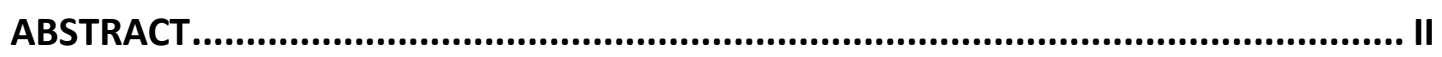

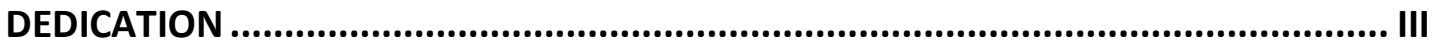

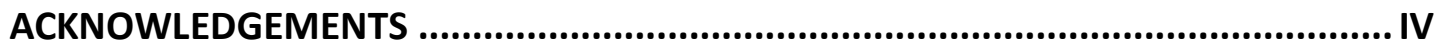

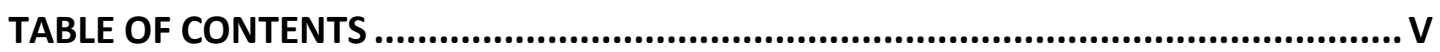

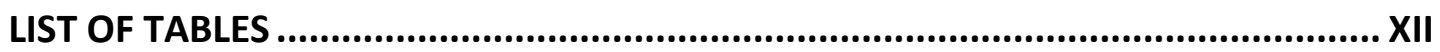

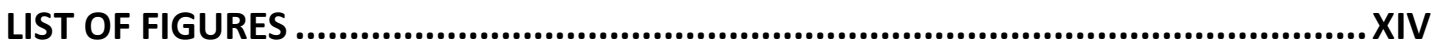

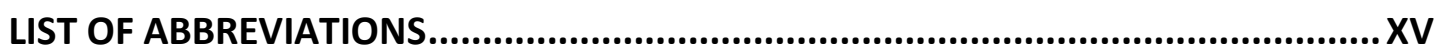

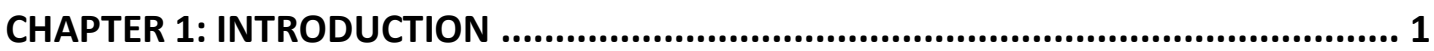

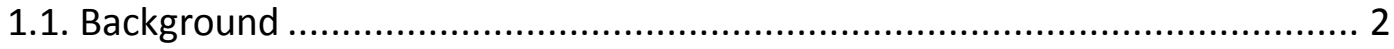

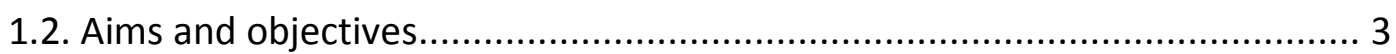

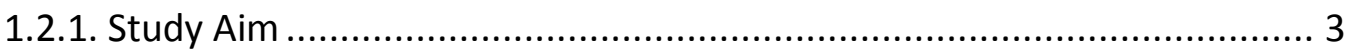

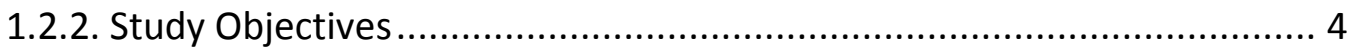

1.3. Suboptimal care as a theoretical lens ..................................................... 4

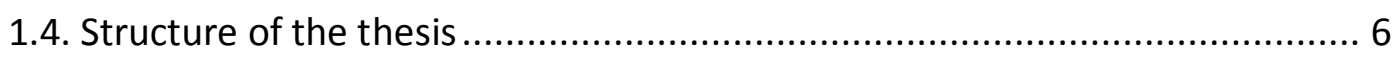

CHAPTER 2: CONCEPT ANALYSIS OF SUBOPTIMAL CARE..................................... 8

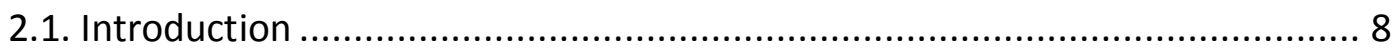

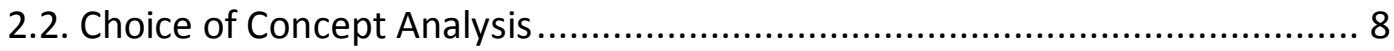

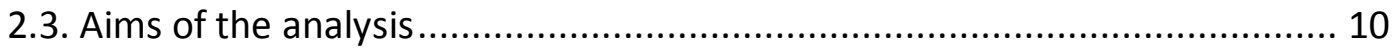

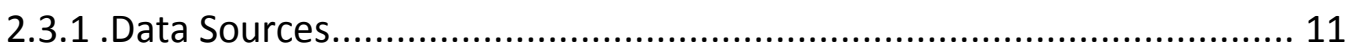

2.4. Definitions and uses of the concept.................................................... 13

2.4.1. Academic disciplines outside of nursing and medicine ....................... 14

2.4.2. The broader nursing and medical context........................................ 14

2.4.3. The care of acutely unwell patients ............................................... 15

2.4.5. Summary of uses of the concept..................................................... 19

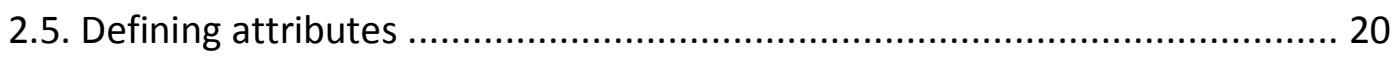

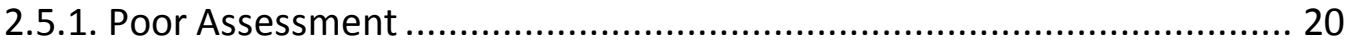

2.5.2. Delays in diagnosis, seeking advice, treatment or referral ................... 21

2.5.3. Inappropriate or inadequate patient management............................ 22 
2.6. Antecedents and Consequences of suboptimal care................................ 23

2.6.1. Patient Complexity .......................................................................... 24

2.6.2. Health Care Workforce ................................................................. 24

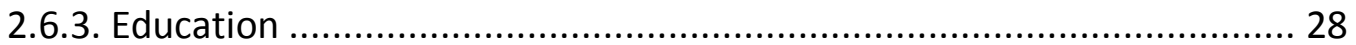

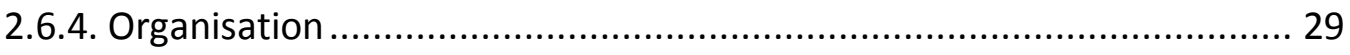

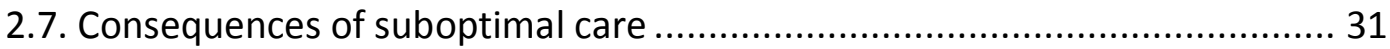

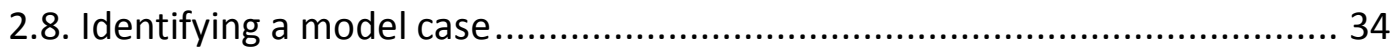

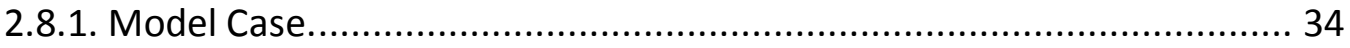

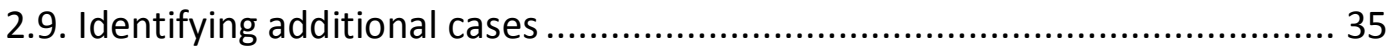

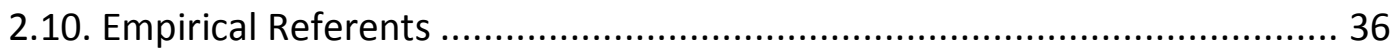

2.11. Methodological limitations of reviewed studies ..................................... 36

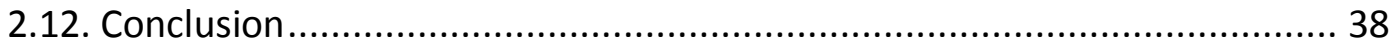

CHAPTER 3 - JUSTIFICATION OF CASE STUDY APPROACH AND RESEARCH DESIGN39

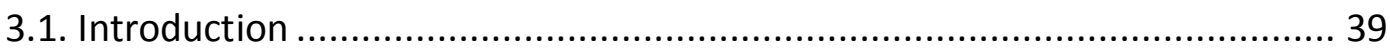

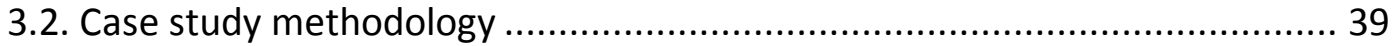

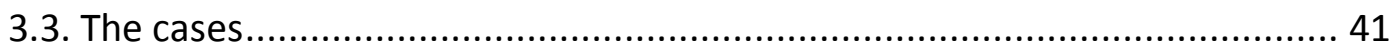

3.4. Determining the type of case study ..................................................... 42

3.5. Limitations of case study research ....................................................... 43

3.6. Developing the research design ............................................................. 43

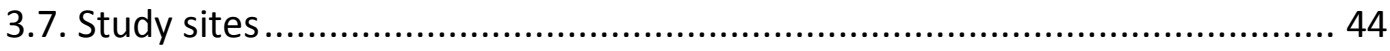

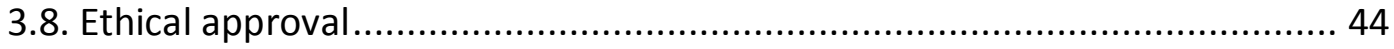

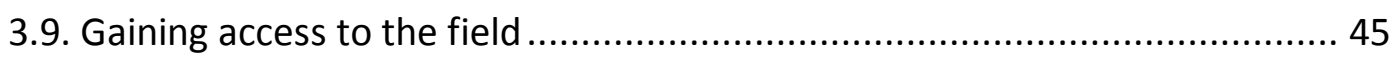

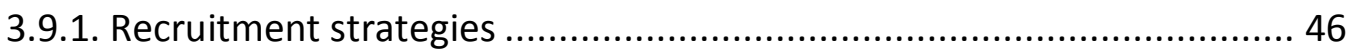

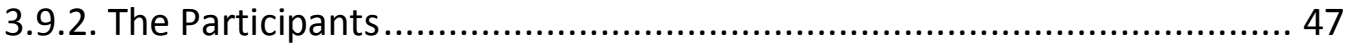

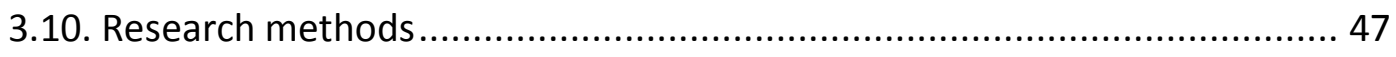

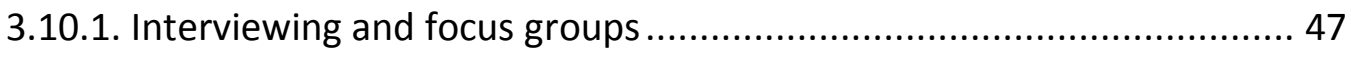

3.10.2. Using documented evidence .................................................... 50

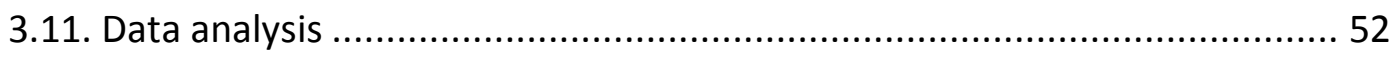

3.11.1. Analysis of the individual data sources - deriving the analytical

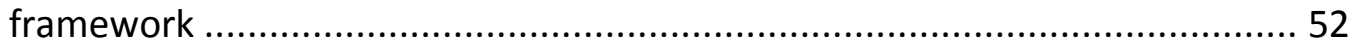

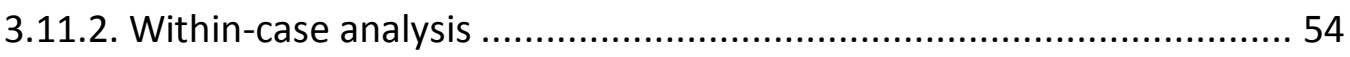




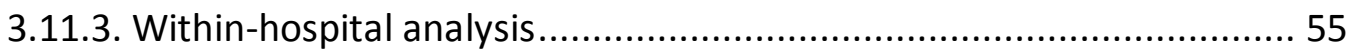

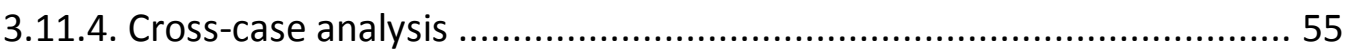

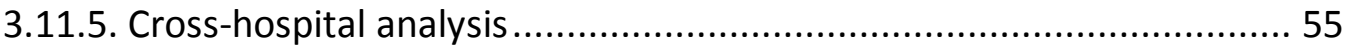

3.12. Ensuring methodological rigour............................................................ 56

3.12.1. Using multiple sources of evidence - methodological triangulation.... 56

3.12.2. Creation of a case study database ............................................. 57

3.12.3. Maintaining a chain of evidence ....................................................... 58

3.12.4. Respondent Validation ........................................................... 58

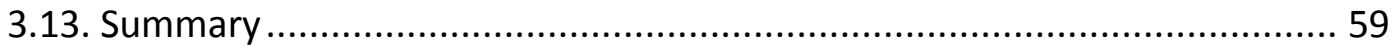

CHAPTER 4: INITIAL FINDINGS: DERIVING THE ANALYTICAL FRAMEWORK...........60

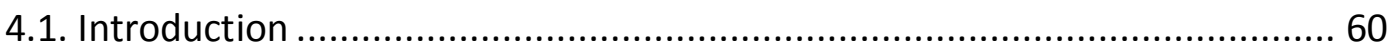

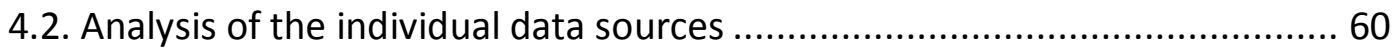

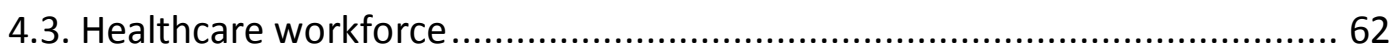

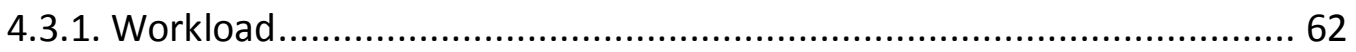

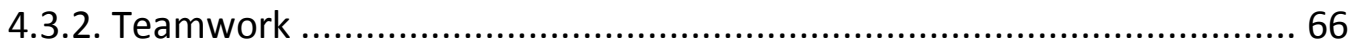

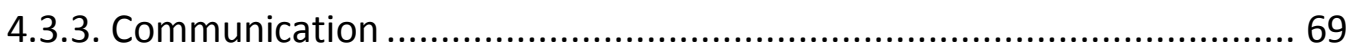

4.3.4. Leadership, accountability and roles.............................................. 72

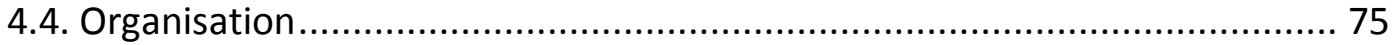

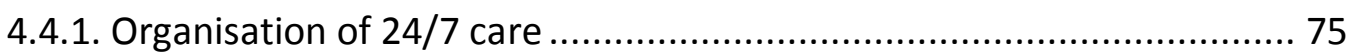

4.4.2. Organisational systems and processes of care .................................. 77

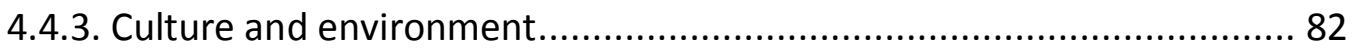

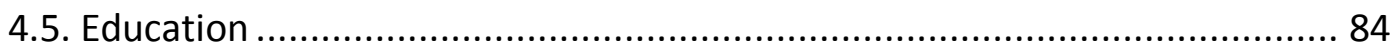

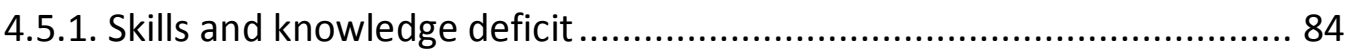

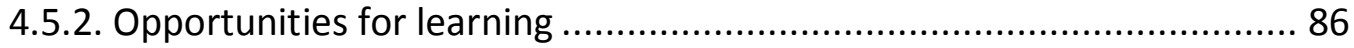

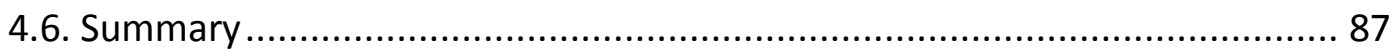

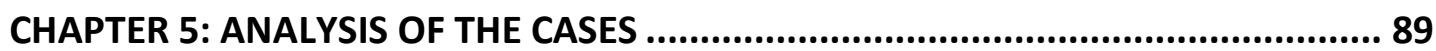

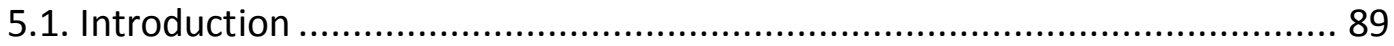

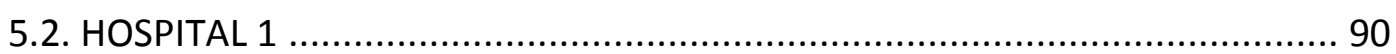

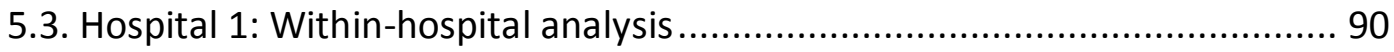

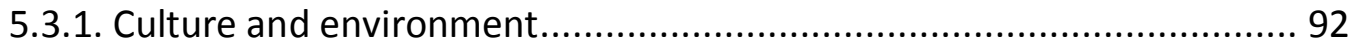

5.3.2. Organisational systems and processes of care ................................. 95 


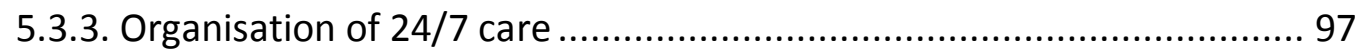

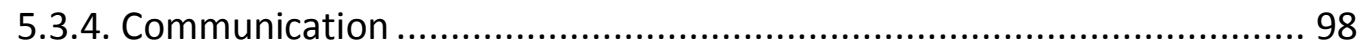

5.3.5. Leadership, accountability and roles.............................................100

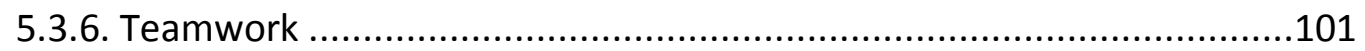

5.3.7. Skills and knowledge deficit ..........................................................102

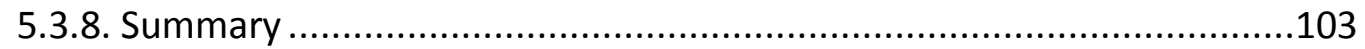

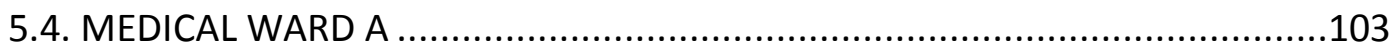

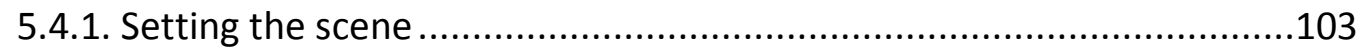

5.5. Medical ward A: Within-case analysis ...................................................105

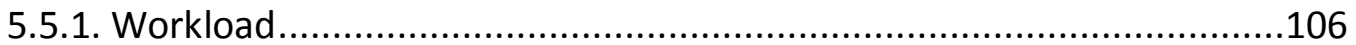

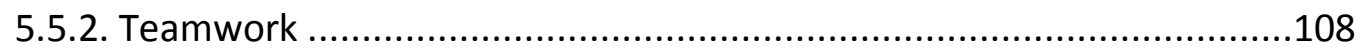

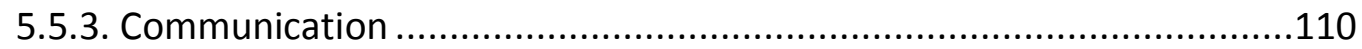

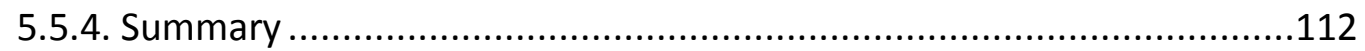

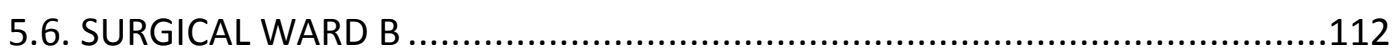

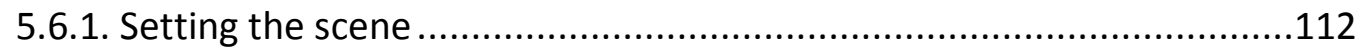

5.7. Surgical ward B: Within-case analysis ..................................................114

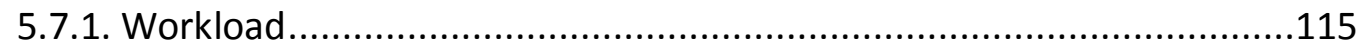

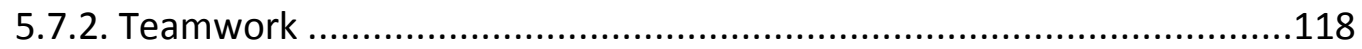

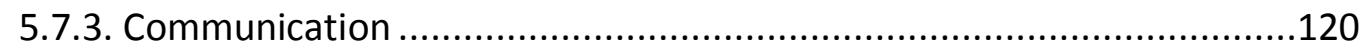

5.7.4. Leadership, roles and accountability.............................................120

5.7.5. Organisational systems and processes of care ................................122

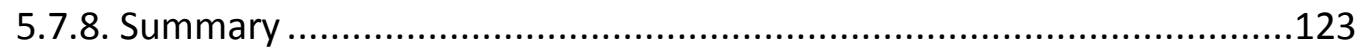

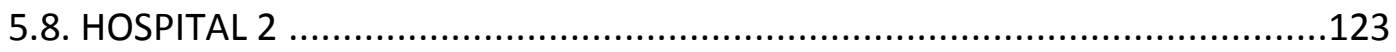

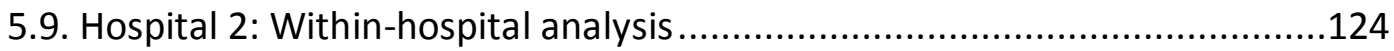

5.9.1. Culture and environment, and organisational systems and processes of

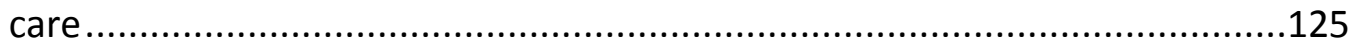

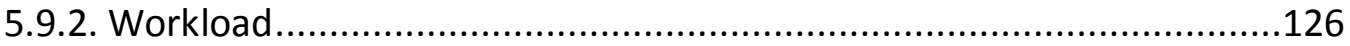

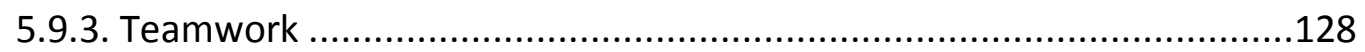

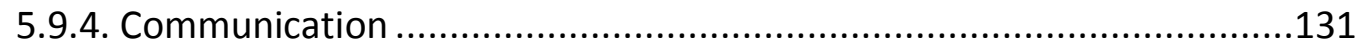

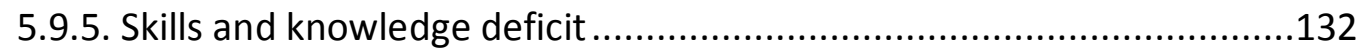

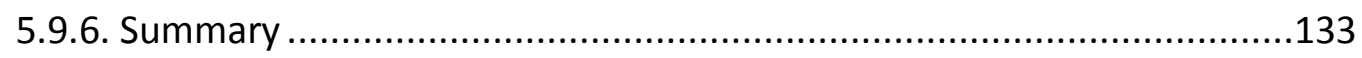




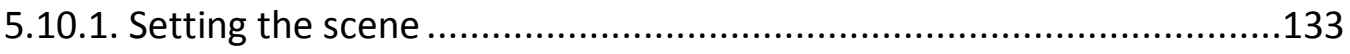

5.11. Medical ward C: Within-case analysis ................................................135

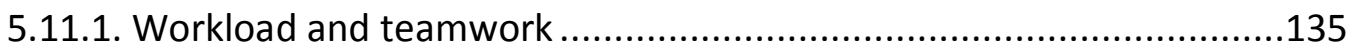

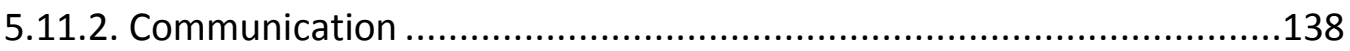

5.11.3. Leadership, roles and accountability.........................................140

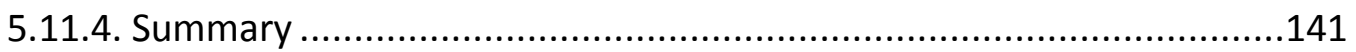

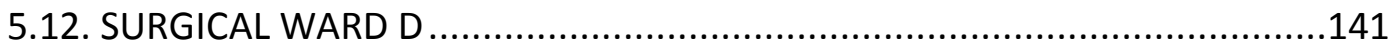

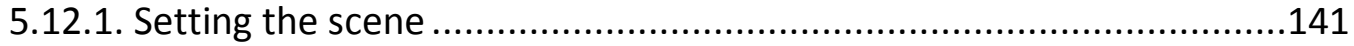

5.13. Surgical ward D: Within-case analysis ...................................................143

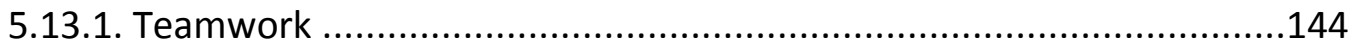

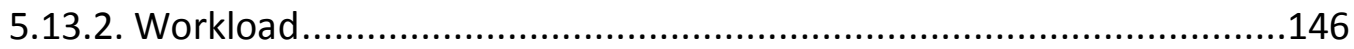

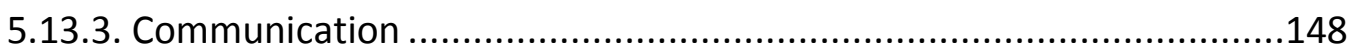

5.13.4. Leadership, accountability and roles...........................................149

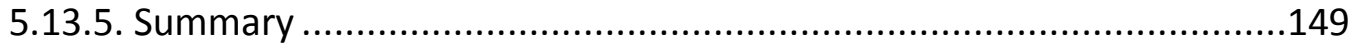

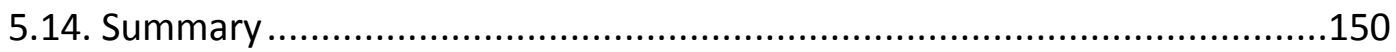

CHAPTER 6: CROSS-CASE AND CROSS-HOSPITAL ANALYSIS .............................151

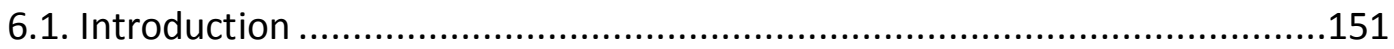

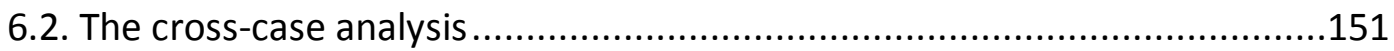

6.3. Analysis of similarities and differences between Medical ward A and Surgical

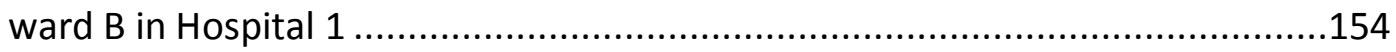

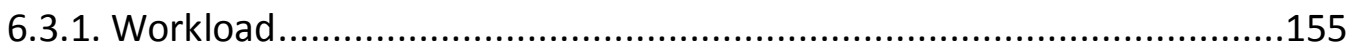

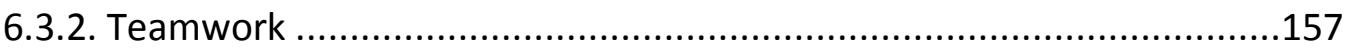

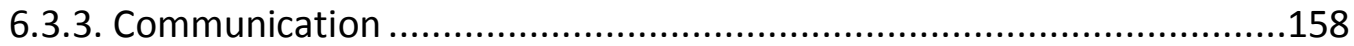

6.3.4. Leadership, accountability and roles............................................158

6.3.5. Organisational systems and processes of care ..................................159

6.4. Analysis of similarities and differences between Medical ward C and Surgical

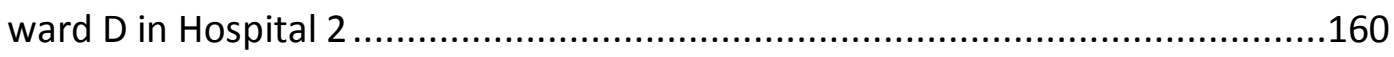

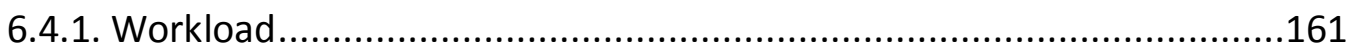

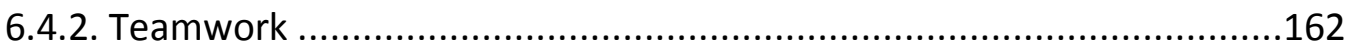

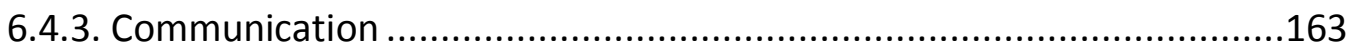


6.5. Analysis of similarities and differences between Medical ward A ..............163

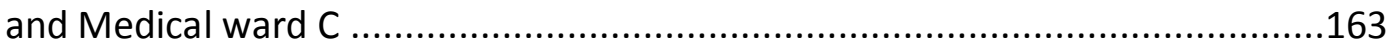

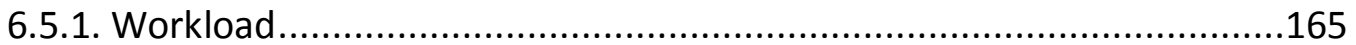

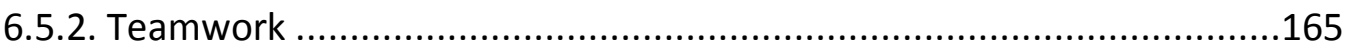

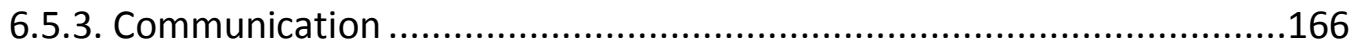

6.5.4. Leadership, accountability and roles................................................166

6.6. Analysis of similarities and differences between Surgical ward B and Surgical ward D

6.6.1. Workload 167

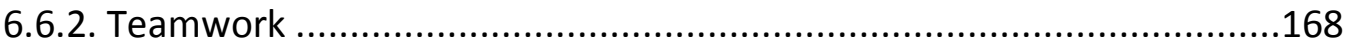

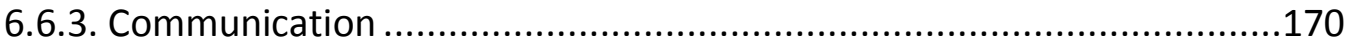

6.6.4. Organisational systems and processes of care ...............................170

6.7. Analysis of similarities and differences in factors between medical and surgical wards in Hospital 1 and Hospital 2 .................................................171

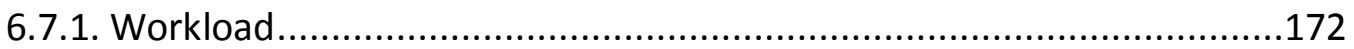

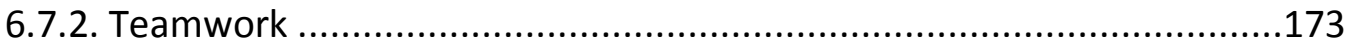

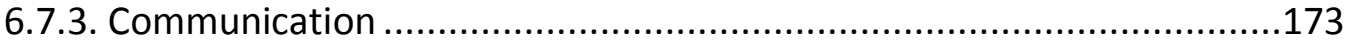

6.7.4. Leadership, accountability and roles...............................................174

6.7.5. Organisational systems and processes of care ..................................174

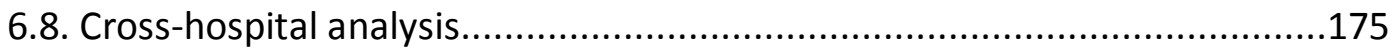

6.8.1. Organisational systems and processes of care .................................176

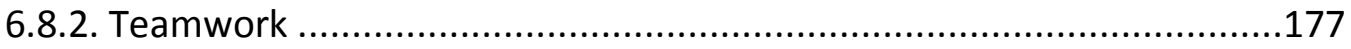

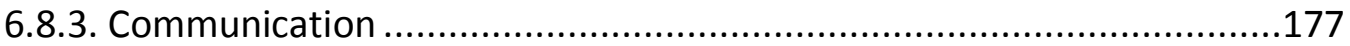

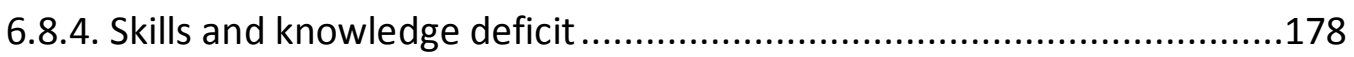

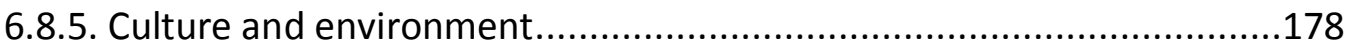

6.8.6. Leadership, accountability and roles............................................178

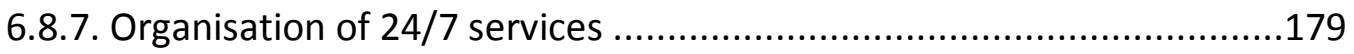

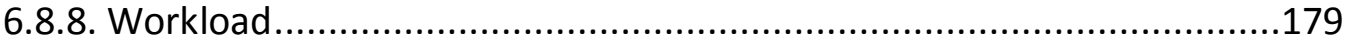

6.9. Similarities and differences between wards and organisational viewpoint regarding factors affecting care of acutely unwell ward patients 


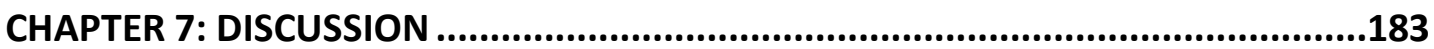

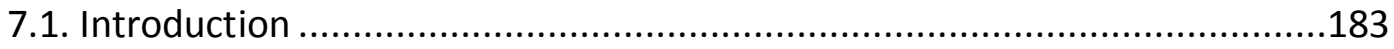

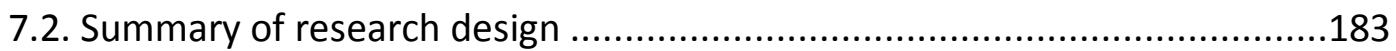

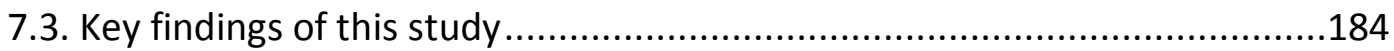

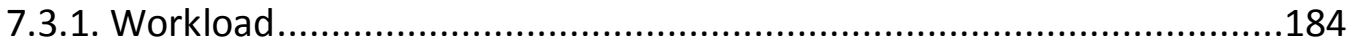

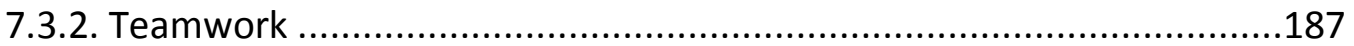

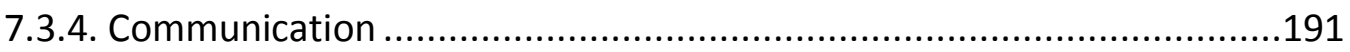

7.3.5. The role of the coordinator............................................................192

7.3.6. Organisational systems and processes of care, and culture and environment

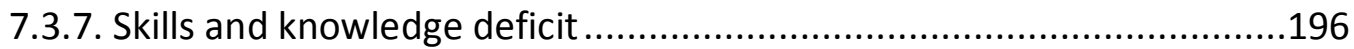

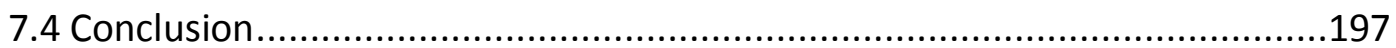

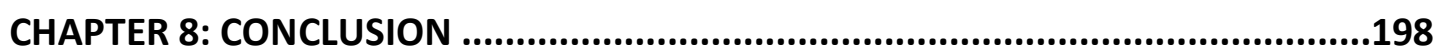

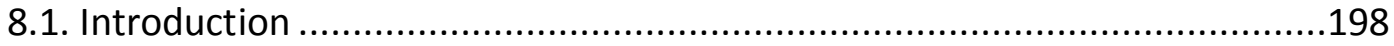

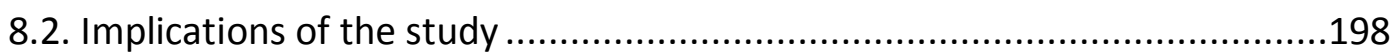

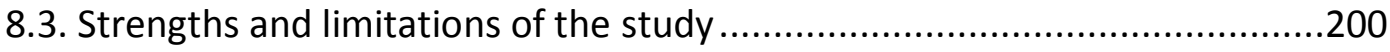

8.4. Recommendations for further research................................................202

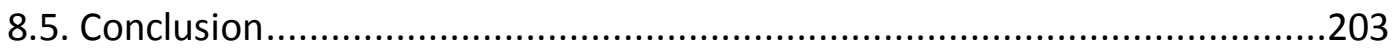

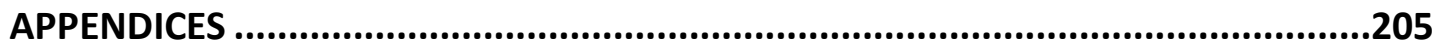

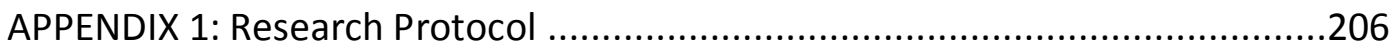

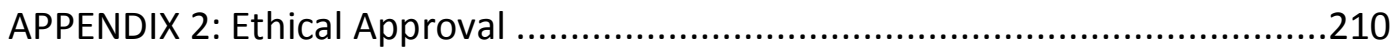

APPENDIX 3: Participant Information Sheet for Stakeholder Interviews ............212

APPENDIX 4: Participant Information Sheet for Stakeholder Focus Groups ......214

APPENDIX 5: Participant Consent Form: DHB Stakeholder Interviews ..............216

APPENDIX 6: Participant Consent Form: DHB Focus Group.............................218

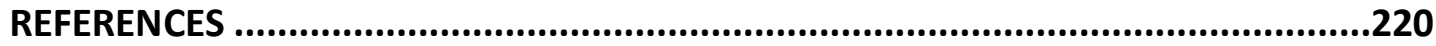




\section{LIST OF TABLES}

Table 1. Three different approaches to concept analysis................................... 9

Table 2. Results of keyword search by database ................................................. 13

Table 3. Articles and reports selected for review to inform the concept analysis ... 17

Table 4. Number of patients suffering consequences related to suboptimal care... 31

Table 5. Breakdown of participants by role and study site ................................. 50

Table 6. Details of all documents reviewed from both hospitals........................... 51

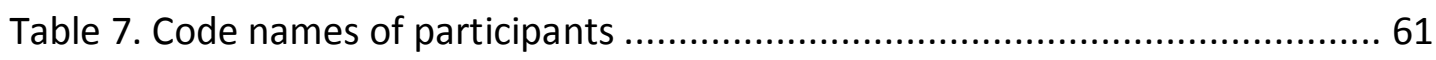

Table 8. Factors to emerge from the theme of workload ..................................... 66

Table 9. Factors to emerge from the theme of teamwork ...................................69

Table 10. Factors to emerge from the theme of communication......................... 72

Table 11. Factors to emerge from the theme of leadership, accountability and roles 75

Table 12. Factors to emerge from the theme of organisation of 24/7 care 77

Table 13. Factors to emerge from the theme of organisational systems and processes of care. 82

Table 14. Factors to emerge from the theme of culture and environment ............ 84

Table 15. Factors to emerge from the theme of skills and knowledge deficit ......... 86

Table 16. Factors to emerge from the theme of opportunities for learning ............ 87

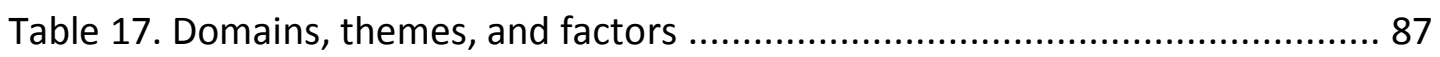

Table 18. Organisational participants - Hospital 1 ............................................. 91

Table 19. Documents reviewed for Hospital 1 ....................................................... 91

Table 20. Domains, themes, and factors Hospital 1 ...........................................103

Table 21. Participants from Medical ward A ......................................................105

Table 22. Documents reviewed for Medical ward A ..........................................106

Table 23. Domains, themes, and factors Medical ward A ..................................112

Table 24. Participants from Surgical ward B ..................................................114

Table 25. Documents reviewed for Surgical ward B..........................................115

Table 26. Domains, themes, and factors Surgical ward B...................................123

Table 27. Organisational participants Hospital 2 ............................................124 
Table 28. Documents reviewed for Hospital 2 ..................................................124

Table 29. Domains, themes, and factors Hospital 2 ........................................133

Table 30. Participants from Medical ward C ...................................................135

Table 31. Documents reviewed for Medical ward C ...........................................135

Table 32. Domains, themes, and factors Medical ward C ..................................141

Table 33. Participants from Surgical ward D .................................................143

Table 34. Documents reviewed for Surgical ward D .........................................143

Table 35. Domains, themes, and factors Surgical ward D .................................150

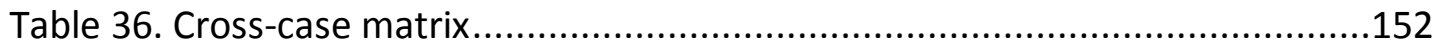

Table 37. Similarities and differences in factors between Wards A and B within Hospital 1

Table 38. Similarities and differences in factors between Wards $C$ and $D$ within Hospital 2 160

Table 39. Similarities and Differences between Medical ward A and Medical ward C

Table 40. Similarities and Differences and between Surgical wards B and Surgical ward D 166

Table 41. Similarities and differences in factors between medical and surgical wards in Hospital 1 and Hospital 2 171

Table 42. Cross-hospital analysis - similarities and differences 175 


\section{LIST OF FIGURES}

Figure 1. Antecedents and defining attributes of suboptimal care 


\section{LIST OF ABBREVIATIONS}

\begin{tabular}{ll} 
ACSQHC & Australian Commission on Safety and Quality in Health Care \\
ACNM & Associate Charge Nurse Manager \\
ALERT & Acute Life Events Recognition and Treatment \\
CCON & Critical Care Outreach Nurse \\
CCOS & Critical Care Outreach Service \\
CCS & Computerised Communication System \\
CMA & Chief Medical Advisor \\
COO & Chief Operating Officer \\
CNE & Clinical Nurse Educator \\
CNM & Clinical Nurse Manager \\
AHSN & After Hours Support Nurse \\
DHB & District Health Board \\
DoN & Director of Nursing \\
EWS & Early Warning Score \\
FTE & Full time equivalent \\
HDC & Health and Disability Commissioner \\
HDU & High Dependency Unit \\
ICU & Intensive Care Unit \\
MET & Medical Emergency Team \\
NCEPOD & National Confidential Enquiry into Patient Outcome and Death \\
NICE & National Institute of Health and Clinical Excellence \\
NPSA & National Patient Safety Agency \\
PDRP & Professional Development and Recognition Programme \\
PRHO & Pre-Ristration House Officer \\
RDA & Resident Doctors Association \\
SBR & Situation, Backround, Assessment, Response/Request \\
\hline
\end{tabular}




\section{CHAPTER 1: INTRODUCTION}

Care of acutely unwell ward patients in New Zealand is receiving increasing attention. Research has demonstrated that the hospital inpatient population is older, more complex, and has increasing numbers of co-morbidities (Resnick \& Marcantonio, 1997; United Nations, 2009; van Weel \& Michels, 1997; World Health Organisation, 2003) leading to a progressively sicker in-hospital patient population. Knowledge of the facts outlined here has precipitated an increasing awareness of the care of sick ward patients and what happens when patient management is not optimal.

A recent Quality Improvement Committee (QIC) report detailing serious and sentinel events from across all District Health Boards (DHB) of New Zealand (NZ) states there are:

many tragic and sad events that have happened to patients in our care. We owe it to them to take every possible step to learn from these events and limit the chance of the recurrence of any similar events. We must be spurred on to encourage open and frank discussion of how these may have happened and to develop even safer health systems that the people of New Zealand can trust. (QIC, 2009, p. 4).

This report demonstrates that the condition of acutely unwell ward patients may deteriorate, leading to catastrophic events including unexpected death or emergency admission to an intensive care unit (ICU). Deterioration that is not recognised or acted upon can lead to delays in diagnosis, treatment, and management (McGloin, Adam, \& Singer, 1999; McQuillan et al., 1998). With such delays having potential catastrophic impact on the management and outcome of acutely unwell ward patients it is clear that this area of study is important for patients, hospital clinicians, hospital managers, and health policy makers. 


\subsection{Background}

Sick ward patients who deteriorate and become acutely unwell ${ }^{1}$ and who experience delays in treatment, diagnosis and management may receive what is termed in the literature 'suboptimal care' (Australian Commission on Safety and Quality in Health Care [ACSQHC], 2010; National Confidential Enquiry into Patient Deaths [NCEPOD], 2005; National Institute for Health and Clinical Excellence [NICE], 2007; NCEPOD, 2009; National Patient Safety Agency [NPSA], 2007). Suboptimal care was first described as a phenomenon in the late 1990s (McGloin, Adam, \& Singer, 1999; McQuillan et al., 1998) and has been further explored through a series of studies from the United Kingdom (UK), Australia and the United States of America (USA). The focus of these studies was to illustrate that patients demonstrate physiological markers of clinical deterioration that were not recognised or that, if recognised, were not acted upon by staff (Franklin \& Mathew, 1994; Kause et al., 2004; McGloin, Adam, \& Singer, 1999; McQuillan et al., 1998; Schein et al., 1990). The organisational factors which may lead to suboptimal care were highlighted but not explored by these studies.

Only a limited number of exploratory studies provide possible explanations for the organisational factors which may lead to the occurrence of suboptimal care (Chellel, Higgs \& Scholes, 2006; Cox, James, \& Hunt, 2006; Cutler, 2002; NPSA, 2007). These UK-based studies suggest that poor communication, increased nursing and medical workloads, inadequate nurse-patient ratios, poor staff skill mix, ineffective teamwork, increased patient acuity, overreliance on machinery, and availability of resources are factors which may cause suboptimal care. To support and expand on these findings two UK reports suggest additional contributing factors such as inexperienced healthcare staff and inadequate assessment of

\footnotetext{
${ }^{1}$ For the purposes of this study an acutely unwell ward patient is defined as a patient whose condition deteriorates unexpectedly. According to the National Institute for Health and Clinical Excellence (2007) this deterioration "can occur at any stage of a patient's illness, although there will be certain periods during which a patient is more vulnerable, such as at the onset of illness, during surgical or medical intervention and during recovery from critical illness" (p. 19).
} 
patients (NCEPOD, 2005; NICE, 2007). Whilst these reports and studies provide an important background to understanding factors affecting care of acutely unwell ward patients, there has not been focussed empirical work exploring organisational factors affecting acutely unwell ward patients in New Zealand or internationally. The value of this study is therefore not dependent on its New Zealand context.

Furthermore there is no empirical work exploring multiple perspectives of clinicians and managers across the organisation from ward to executive level. Of the research focussing on factors affecting care of acutely unwell patients, nurses were the only participants in two studies (Cox et al., 2006; Cutler, 2002) whereas nurses, doctors and some managers were participants in one study (NPSA, 2007). There were no studies which deliberately explored the views of executive-level staff to provide an organisational perspective. This indicates a deficit in the knowledge base which seeks to understand why suboptimal care occurs.

It is clear that the factors affecting care of the acutely unwell patient have received minimal attention internationally. Furthermore, there has been no research with this focus carried out in New Zealand. The concern is that unless factors affecting care of acutely unwell ward patients are clear, it will be difficult to prevent suboptimal care from occurring. Given the potentially devastating consequences of suboptimal care for acutely unwell ward patients this is a serious omission both internationally and in the New Zealand context.

\subsection{Aims and objectives}

The previous section has outlined the limited research that has explored factors affecting the care of acutely unwell ward patients and presented the case for further study in this area. It also demonstrated that suboptimal care of acutely unwell ward patients is a complex and multifaceted issue.

\subsubsection{Study Aim}

The aim of this study was to determine factors affecting care of acutely unwell ward patients in New Zealand. This study also investigated whether factors affecting care of acutely unwell ward patients were unique to particular contexts, for example a particular ward within a hospital, or common across wards or 
hospitals. Furthermore, seeking the viewpoints of healthcare staff in different roles within an organisation gave a broader perspective.

Drawing on the experiences of ward nurses, doctors, and hospital managers, the aim of this study was to establish the organisational factors which impeded or supported care within a general surgical or medical ward of acutely unwell patients.

\subsubsection{Study Objectives}

Two research objectives were developed to explore these areas. These were:

- To determine whether factors affecting care of the acutely unwell ward patient were unique to specific contexts

- To identify whether multiple perspectives existed within an organisation regarding factors affecting the care of acutely unwell ward patients.

Because the context in which acutely unwell ward patients are cared for (both at a ward and wider organisational level) was central to the aim of this study, that context influenced the choice of methodological approach. Case study research is recognised as being appropriate when the researcher specifically wants to explore contextual conditions because the boundaries between the context and the phenomenon under investigation are not clear (Yin, 2003). Using a multiple case study approach for this study allowed factors affecting care of acutely unwell ward patients to be explored in the context in which they occurred across several different locations. This allowed for the identification of any recurrent themes across the study sites.

\subsection{Suboptimal care as a theoretical lens}

Healthcare research has demonstrated that in order to provide optimal care there needs to be an adequate number of well-educated, well organized health care professionals making the correct diagnosis, prescribing and providing the correct care and treatment whilst offering continuity across and within teams (Aiken, Clarke, Sloane, Sochalski,\& Silber, 2002; Ferlie \& Shortell, 2001;Tourangeau et al., 2006). Additionally using evidence based guidelines, ensuring positive nursedoctor relationships and commitment from hospital senior management teams to 
quality systems and processes and patient safety have all been demonstrated as key to the provision of optimal care (Clarke \& Aiken, 2006; Donabedian, 2004) However focus in recent years has been on what leads to suboptimal care as a way of defining and enhancing optimal care.

As described in 1.1, 'suboptimal' is a term frequently applied to the care of acutely unwell ward patients in the literature. The suboptimal care literature argues that caring for the acutely unwell ward patient is affected by the potential multiple factors affecting the patient and the complex interrelationships between those factors which in turn may be influenced by the organisation, health care workforce issues and a lack of education. Using the suboptimal care literature as a theoretical lens for this study will allow the multifaceted and complex nature of caring for the acutely unwell ward patient to be fully explored.

However while the term 'suboptimal care' is used frequently in research related to acutely unwell ward patients, the meaning is not often made explicit. Examining how suboptimal care has been applied as a concept within the literature is key to identifying factors affecting the care of acutely unwell ward patients. One way of clarifying a concept is through concept analysis. The purpose of a concept analysis is to provide the researcher with a basic understanding of the important attributes of a concept. By understanding these attributes the researcher can define the problem more clearly, which will aid construction of robust research designs and instruments for data collection (Walker \& Avant, 2005).

Clarifying attributes of a concept (in this instance through the concept analysis) is especially important when using case study methodology. Yin (1993) argues that "the term theory covers more than causal theories. Theory means the design of research steps according to some relationship to the literature, policy issues, or other substantive source" (Yin, 1993, p. 4). Within all types of case study, theory also informs the choice of cases to be studied when following a replication or multiple case design (Yin, 1993). Finally, the correct specification of a theory will provide the researcher with a predicted pattern of events. This pattern then becomes a series of bench marks against which the actual data can be compared when conducting data analysis (Yin, 1993). 
The concept analysis of suboptimal care (presented as Chapter 2) using Walker and Avant's (2005) approach will identify uses of the concept within the literature to determine the selection of the 'cases' for this research. Furthermore, the antecedents of suboptimal care will provide the initial overall coding categories for the analysis of the data.

\subsection{Structure of the thesis}

This thesis is presented in eight chapters. This chapter has outlined the background to the study, scope of the work, and set the scene for the multiple case study undertaken.

Chapter 2 demonstrates the lack of research identifying factors affecting care of acutely unwell ward patients. What is evident in the literature is that the term 'suboptimal care' is frequently used to describe the care of acutely unwell ward patients. In order to reveal current understanding of factors affecting care of the acutely unwell ward patients a concept analysis of suboptimal care is undertaken and presented in Chapter 2. Through this concept analysis, defining attributes and antecedents of suboptimal care are identified. In this way suboptimal care is the theoretical lens for the study.

In Chapter 3, the antecedents emerging from the concept analysis of suboptimal care more clearly define the research problem and are used to inform the research design and data collection instruments (Walker \& Avant, 2005). Chapter 3 then presents and justifies the case-study approach, the selection of cases and participants, and procedures used in the collection and analysis of data.

Consistent with case-study approach to data analysis and presentation of findings, the study findings are multi-level and presented as Chapters 4, 5 and 6. The data findings from initial analysis of the data from both organisations and all four cases are presented in Chapter 4. The analytical framework derived from this stage of analysis then informs analysis of subsequent data. This is presented in Chapter 5, where within-case and within-hospital case comparisons are presented. Chapter 6 presents the final level of findings to emerge from this study in cross-case and cross-hospital case comparisons, enabling exploration of similarities and differences between wards and hospitals. 
Chapter 7 provides a platform on which the findings from this study are discussed alongside the current evidence base. Through this the contribution of the study to our understanding of factors affecting acutely unwell ward patients is made explicit. This is further developed in Chapter 8, where, while recognising the limitations of this study, the implications of this research for clinical practice, national policy, theoretical development, and future research activities are presented. 


\section{CHAPTER 2: CONCEPT ANALYSIS OF SUBOPTIMAL CARE}

\subsection{Introduction}

Chapter 1 has demonstrated that, to date, the dominant focus within the literature has been quantifying the occurrence and consequences of suboptimal care, with minimal research about the organisational factors which cause it. Despite the term 'suboptimal' frequently being used to describe the care of acutely unwell ward patients, the term remains inadequately defined.

In order to address what factors cause suboptimal care, a clearer understanding of the term 'suboptimal care' is required. Defining suboptimal care is a first step to enabling health care professionals to communicate effectively about what causes it and how it may be prevented. A clearer understanding of the term will also inform the design and analytical framework of this study. One way of clarifying the term 'suboptimal care' is through the process of concept analysis.

This chapter has as its focus a concept analysis of suboptimal care of acutely unwell ward patients. Through identification and description of definitions, uses, attributes, antecedents, and consequences of suboptimal care, the meaning of this concept is examined. For this study Walker and Avant's (2005) structured approach was used to ensure a rigorous review of the literature.

\subsection{Choice of Concept Analysis}

There are several approaches that can be used to conduct a concept analysis (Rodgers, 1989; Walker and Avant, 2005; Wilson, 1963). Each of these has its own ontological $^{2}$ perspective, being either realist ${ }^{3}$ or relativist $^{4}$ in orientation. For example, Wilson's ontological perspective is relativist and context based. In contrast, Rodgers' and Walker and Avant's realist perspective minimizes the relationship between contexts and concepts. Table 1 illustrates the three main

\footnotetext{
${ }^{2}$ Ontology is defined as questioning the nature of being or reality (Denzin \& Lincoln, 1994).

${ }^{3}$ The realist perspective purports "that a definite world structure exists" (Polifroni \& Welch, 1999, p. 128).

${ }^{4}$ A relativist viewpoint states that there are multiple realities (Denzin \& Lincoln, 1994).
} 
approaches identified in the literature and demonstrates their similarities and differences.

Table 1. Three different approaches to concept analysis

\begin{tabular}{|c|c|c|c|}
\hline Criteria & $\begin{array}{l}\text { Wilson }(1963, \text { p. } 23- \\
26)\end{array}$ & Rodgers (1989, p.333) & $\begin{array}{l}\text { Walker and Avant } \\
(2005, \text { p. 65) }\end{array}$ \\
\hline $\begin{array}{c}\text { Number of } \\
\text { steps }\end{array}$ & 11 & 7 & 8 \\
\hline Process & $\begin{array}{l}\text { 1.Isolate questions } \\
\text { of concepts } \\
\text { 2.Write answers } \\
\text { 3.Model answers } \\
\text { 4.Related cases } \\
\text { 5.Contrary cases } \\
\text { 6.Borderline cases } \\
\text { 7.Invented cases } \\
\text { 8.Social context } \\
\text { 9.Underlying anxiety } \\
\text { 10.Practical results } \\
\text { 11.Results in } \\
\text { language }\end{array}$ & $\begin{array}{l}\text { 1.Identify and name } \\
\text { the concept of } \\
\text { interest } \\
\text { 2.Identify surrogate } \\
\text { terms and relevant } \\
\text { uses of the concept } \\
\text { 3.Identify and select } \\
\text { an appropriate realm } \\
\text { (sample) for data } \\
\text { collection } \\
\text { 4.Identify the } \\
\text { attributes of the } \\
\text { concept } \\
\text { 5.Identify the } \\
\text { references, } \\
\text { antecedents and } \\
\text { consequences of the } \\
\text { concept if possible } \\
\text { 6.Identify related } \\
\text { concepts } \\
\text { 7.Identify a model } \\
\text { case of the concept }\end{array}$ & $\begin{array}{l}\text { 1.Select a concept } \\
\text { 2.Determine the aims } \\
\text { and purposes of the } \\
\text { analysis } \\
\text { 3.Identify all the uses of } \\
\text { the concept that you } \\
\text { can discover } \\
\text { 4.Determine the } \\
\text { defining attributes } \\
\text { 5.Identify a model case } \\
\text { 6.Identify borderline, } \\
\text { related, contrary, } \\
\text { invented and } \\
\text { illegitimate cases } \\
\text { 7.Identify antecedents } \\
\text { and consequences } \\
\text { 8.Define empirical } \\
\text { referents }\end{array}$ \\
\hline $\begin{array}{l}\text { Ontological } \\
\text { orientation }\end{array}$ & Relativist perspective & Realist perspective & Realist perspective \\
\hline $\begin{array}{l}\text { Key } \\
\text { Differences }\end{array}$ & - Context based & 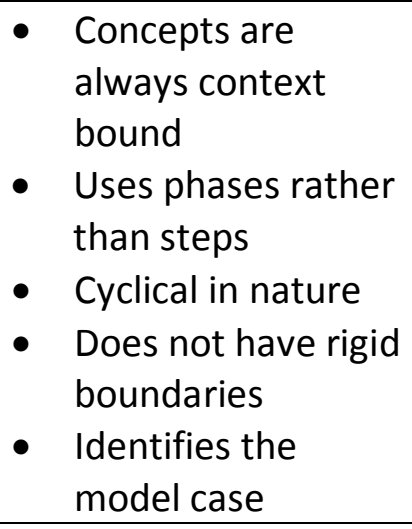 & $\begin{array}{l}\text { - Steps are iterative } \\
\text { - Constructs the } \\
\text { model case } \\
\text { - Uses terms concept } \\
\text { and variable } \\
\text { interchangeably }\end{array}$ \\
\hline
\end{tabular}


Of these three approaches, Walker and Avant's (2005) was chosen. This approach is the most frequently used type of concept analysis in nursing theory development (Duncan, Cloutier, \& Bailey, 2007; Hupcey \& Penrod, 2005; Weaver \& Mitcham, 2008). Walker and Avant state that the purpose of a concept analysis is to operationalise a phenomenon so that it can be used for theory development and/or research measurement and suggest that failure to have a systematic process for variable development jeopardises the validity of future research.

However several authors have criticized Walker and Avant's (2005) linear, positivist and reductionist approach, citing its inability to determine the level of a concept's maturity, insufficient attention to the theoretical context from which the concept is derived and difficulties with applicability of the findings (Baldwin, 2008; Hupcey \& Penrod, 2005; Paley, 1996; Rodgers, 1989; Weaver \& Mitcham, 2008). Risjord (2009) however provides an alternative perspective by proposing that concepts can be related to contexts "such as theories, discourses, or speech communities" (p. 685). After consideration of these comments, Walker and Avant's approach was identified as the best fit for this study.

Walker and Avant's (2005) approach, with its eight stages (Table 1) was chosen specifically because of its realist perspective and systematic approach (Walker \& Avant, 2005) and its ease of use by novice concept analysts (Brennan, 1997). Also it allows for identification of antecedents to the concept which are critical to the structure of this research.

\subsection{Aims of the analysis}

In order to direct the concept analysis a clear aim was determined. The aim of the concept analysis was to clarify the meaning of the concept 'suboptimal care'. Of specific interest to this study was a thorough exploration of antecedents to suboptimal care. These are the factors affecting care of acutely unwell ward patients that have previously been determined through empirical enquiry. In line with Walker and Avant's (2005) approach, a literature search was undertaken to identify all uses of the term 'suboptimal care'.

Walker and Avant's approach encourages authors to consider all uses of the term and not to be restricted to the nursing and medical literature. This, they 
believe, will prevent bias in understanding of the nature of the concept. Furthermore, by conducting a thorough review of the literature the choice of the defining attributes can be supported and validated (Walker \& Avant, 2005).

\subsubsection{Data Sources}

The literature search was conducted in three distinct phases. Firstly, databases from outside the field of medicine and nursing were searched using the search terms 'suboptimal' and 'sub-optimal' to see how the term was used in other academic disciplines. Databases searched were Proquest (computer sciences, economics, and bioinformatics), CSA Illumina (engineering and social sciences), and Mathscinet and lecture notes in mathematics (mathematics). A total of eight abstracts from these three databases were randomly ${ }^{5}$ selected for review to establish a general overview of how these disciplines used the term.

The second phase of the literature search involved searching the Cumulative Index Nursing and Allied Health (CINAHL), Cochrane and Medline databases using the terms 'suboptimal', 'sub-optimal', and 'suboptimal care'. As with the literature from academic disciplines outside of nursing and medicine, a random ${ }^{6}$ selection of 16 abstracts was reviewed to provide a general understanding of how the term was used in the broader nursing and medical literature.

The third and final phase of the search was to focus specifically on suboptimal care of the acutely unwell patient. CINAHL, Cochrane, and Medline databases were searched using the original search terms 'suboptimal care', 'sub-optimal', and 'sub optimal'. Supplementary search terms, 'acutely ill patients', 'acutely ill ward patients', 'physiological deterioration', 'inadequate care', 'substandard care', and 'poor quality care', were entered to ensure that all information related to suboptimal care of the acutely unwell patient was reviewed. Where initial search terms yielded a large number of hits search terms were combined; for example,

\footnotetext{
${ }^{5}$ Randomisation was done by selecting every $10^{\text {th }}$ abstract from the first 80 abstracts to be listed in the search results (30 from Proquest; 30 from CSA illumina; 20 from Mathscinet and lecture notes in mathematics).

${ }^{6}$ Randomisation was done by selecting every $10^{\text {th }}$ abstract from the first 160 articles to be listed in the search results.
} 
'suboptimal care' and 'acutely ill patients'. In addition, Boolean functions, truncation searches, and reference lists from relevant publications were reviewed. No date or language restrictions were imposed. Only articles relevant to suboptimal care of the acutely unwell adult ward patient were included. Papers had to meet one of the following conditions to be in the final concept analysis: suboptimal care of acutely unwell ward patients was an outcome measure of the study; the study of factors contributing to suboptimal care was the core content of the paper; or service initiatives to prevent suboptimal care were measured as part of the study. Articles included were qualitative and quantitative research papers, literature reviews and one editorial.

The initial search for this study was run in 2007 and repeated in 2010. The 2007 search yielded 39 articles which were included in the initial analysis. The 2010 search yielded 49 articles and the concept analysis was updated to reflect these ten additional articles.

Grey literature sources (patient-safety-related government agency websites) were searched and yielded five documents from government agencies (National Patient Safety Agency, Modernisation Agency, National Institute of Health and Clinical Excellence, Australian Commission on Safety and Quality in Health Care). This provided a further five sources for the concept analysis. No books or dissertations were included in the final analysis. The majority of the literature came from the United Kingdom (UK), Australia, and United States of America (USA). Furthermore, the context (when made explicit) for the majority of the research was located in general medical and surgical inpatient settings. All literature reviewed was in English and was published between 1990 and 2010. Table 2 demonstrates the number of hits per search term by database and the number of reviewed abstracts. 
Table 2. Results of keyword search by database

\begin{tabular}{|l|l|l|l|l|}
\hline \multicolumn{2}{|l|}{ Search Term } & Medline & Cochrane & $\begin{array}{l}\text { Total } \\
\text { number of } \\
\text { screened } \\
\text { abstracts }\end{array}$ \\
\hline $\begin{array}{l}\text { Suboptimal } \\
\text { care/suboptimal } \\
\text { ward care }\end{array}$ & 128 & 2951 & 4 & 13 \\
\hline $\begin{array}{l}\text { Critically ill ward } \\
\text { patients }\end{array}$ & 5 & & & \\
\hline Acutely ill patients & 307 & 169 & 25 & 13 \\
\hline $\begin{array}{l}\text { Acutely ill ward } \\
\text { patients }\end{array}$ & 4 & 2138 & 7 & 15 \\
\hline Inadequate care & 505 & 38 & 5 & 2 \\
\hline Substandard care & 108 & 12848 & 25 & 8 \\
\hline Poor quality care & 165 & 485 & 6 & 8 \\
\hline $\begin{array}{l}\text { Physiological } \\
\text { deterioration }\end{array}$ & 25 & 8858 & 155 & 16 \\
\hline Total & & 1494 & 5 & 9 \\
\hline
\end{tabular}

To correspond with the three phases of the literature search, uses of the concept are presented in the following sections; academic disciplines outside of nursing and medicine; the broader nursing and medical context; the care of acutely unwell patients. In line with Walker and Avant's (2005) approach, online dictionaries (AskOxford.com, 2009; Cambridge online dictionaries, 2009) were also searched for definitions of the term.

\subsection{Definitions and uses of the concept}

The search of online dictionaries provided only one definition which defined suboptimal as "of less than the highest standard or quality" (AskOxford.com, 2009). Search in medical dictionaries did not yield any further definitions (MedicineNet.com, 2009; MedlinePlus, 2009). There were no synonyms for suboptimal care. 'Suboptimal' is a compound word which may explain why there was no definition in three of the four dictionaries searched (Cambridge online dictionaries, 2009; MedicineNet.com, 2009; MedlinePlus, 2009). On reflection, using dictionaries to provide definitions may be useful in concepts where there is more than one known definition. 


\subsubsection{Academic disciplines outside of nursing and medicine}

Exploring literature from databases outside the field of medicine and nursing was not helpful in terms of overall understanding of the term. Within the fields of bioinformatics, computer sciences, mathematics and engineering the term 'suboptimal' is associated with a continuum whereby optimal solutions are the desired endpoint or sequence. Alignments between variables are mapped to discover how close to the optimal solution the suboptimal solution occurs. This allows the relationship between the optimal and suboptimal solutions to be determined (Byers \& Waterman, 1984; Costanza, 2007; Joo, Lee, Kim, Jong Lee, \& Lee, 2008; Sinclair, Prazenica \& Jeffcoat, 2008). In these disciplines accepting a suboptimal solution does not constitute a negative outcome, with suboptimal solutions often being pragmatic choices within the right context. This is at odds with healthcare, where a suboptimal solution is not an acceptable option as it may have a detrimental effect on patient outcome.

Finally within the economic literature the term suboptimal was used in conjunction with the Pareto principle. Pareto optimality is a relational concept in which "a given economic arrangement is efficient if there can be no arrangement which will leave someone better off without worsening the position of others " (Brownstein, 1980, p. 94). As a result any exchange or reallocation of resources is Pareto suboptimal if the exchange or reallocation will cause harm to others (Gibbard, 1984). This relational aspect of the term suboptimal is important to consider in healthcare given that the reallocation of resources from one area of care may impact on care delivery and ultimately patient outcome elsewhere.

\subsubsection{The broader nursing and medical context}

In the medical and nursing literature suboptimal is associated with poor quality of care. This can be due to lack of knowledge (Gershenson, 2001); lack of adherence to guidelines or best practice (Palmer, Appleton, \& Rodrigues, 2003; Sheilds, Comstock, \& Weiss, 2004) and disagreement between physicians in diagnostic approach (Konijnenberg et al., 2004). Thus the term suboptimal is associated with negative connotations in the broader healthcare context. 


\subsubsection{The care of acutely unwell patients}

In the context of the acutely unwell ward patient the concept of suboptimal care is relatively new. The concept of suboptimal care first emerged within the reviewed literature in 1998. The early research focussed on recognition, quantification, and subsequent management of clinical indicators of suboptimal care. McQuillan et al. (1998) carried out a prevalence study which examined the nature, attributes, and consequences of suboptimal care by evaluating the quality of care prior to admission to intensive care in the UK. Suboptimal care was determined by two assessors who rated quality of medical care and appropriateness and timeliness of admission to intensive care. Assessors were specifically asked to score the adequacy of management of oxygen therapy, airway, breathing, circulation, and monitoring using a linear visual analogue scale. Of 100 patients studied, the assessors agreed that 54 received suboptimal care. This study has since been criticized for its lack of definition of suboptimal care (McArthurRouse, 2001). Without a clear definition, it is argued, assessment of what was deemed to be suboptimal care was subjective and relied upon assessor opinion. As a result this study could have either over- or under-reported the extent of suboptimal care. Despite this criticism, McQuillan et al's study features regularly in the literature reviewed as evidence of the existence and nature of suboptimal care.

A second frequently cited study which focuses directly on quantifying suboptimal care is a UK audit carried out over six months to determine the incidence of unexpected deaths on the wards and whether they were potentially avoidable. This study differs from McQuillan et al. (1998) in two ways. Firstly the investigators presented a clear definition, defining suboptimal care as non-recognition of an abnormality clearly apparent from physiological recordings or laboratory data but which had either not been identified in the case records or not acted upon with any obvious therapeutic intervention (i.e. no entry on the drug chart) or clearly inappropriate or inadequate treatment, although the case records showed that the abnormality had been identified by nursing or medical staff (McGloin, Adam \& Singer, 1999, p. 256). 
Secondly, assessors were blinded to the outcome and initial diagnosis of patients, thus increasing the methodological rigour of the study. The study identified $36 \%(31 / 86)$ of patients as receiving suboptimal care.

In the reviewed literature suboptimal care is frequently associated with the terms 'avoidable' and 'preventable'. Studies which examine the incidence of abnormalities in vital signs preceding cardiac arrest have demonstrated physiological deterioration in patients within six to eight hours of cardiac arrest (Franklin \& Mathew, 1994; Schein, Hazday, Pena, Ruben, \& Sprung, 1990). Both of these retrospective chart reviews conducted in the USA conclude that cardiac arrests are not sudden events and note "that the problem is commonly not the absence of pertinent information, but the clinical response to that information" (Franklin \& Mathew, 1994, p.247). Key findings of such papers are that cardiac arrests are avoidable if deteriorating vital signs are acted upon.

These key findings are supported by three retrospective chart reviews of patients who suffered a cardiac arrest or unplanned admission to an intensive care unit (ICU) that investigated the nature and duration of clinical abnormalities in patients prior to the critical event (Buist et al., 1999; Goldhill, White, \& Sumner 1999; Hodgetts et al., 2002). All three studies recommend strategies to identify clinically unstable patients early so that any abnormalities in vital signs can be acted upon in a more timely manner such as using Medical Emergency Teams (MET). The focus of these studies was to demonstrate the avoidable and preventable nature of suboptimal care by identifying that physiological deterioration was not detected. These studies did not determine the reasons why such clinical instability was not recognised.

How the term suboptimal is used in the remainder of the reviewed literature draws significantly from either the definition given by McGloin et al. (1999) or the description presented by McQuillan et al. (1998). For example, the only literature review which seeks to identify the factors affecting suboptimal care in the acutely unwell ward patient states that suboptimal care implies "lack of knowledge relating to the significance of clinical findings relating to dysfunction of airway breathing and circulation or problems related to system failures that inhibits care delivery" 
(Massey, Aitken, \& Chaboyer, 2008, p. 128). This is similar to the description provided by McQuillan et al. (1998).

Table 3 lists the articles and reports reviewed for this concept analysis where suboptimal care of acutely unwell ward patients was an outcome measure of the study; factors contributing to suboptimal care were the core content of the paper; or service initiatives to prevent suboptimal care were measured as part of the study.

Table 3. Articles and reports selected for review to inform the concept analysis

\begin{tabular}{|c|c|}
\hline \multicolumn{2}{|c|}{ Research } \\
\hline Study Focus & Authors \\
\hline $\begin{array}{l}\text { The contribution and effectiveness of } \\
\text { outreach services or medical emergency } \\
\text { teams }\end{array}$ & $\begin{array}{l}\text { 1. Bagshaw et al., } 2010 \\
\text { 2. Ball, Kirby, \& Williams, } 2003 \\
\text { 3. Bell, Konrad, Granath, Ekbom, \& } \\
\text { Martling, } 2005 \\
\text { 4. Chellel, Higgs, \& Scholes, } 2006 \\
\text { 5. Hillman et al., } 2005 \\
\text { 6. Jones et al., } 2006 \\
\text { 7. Lee, Bishop, Hillman, \& Daffurn, } \\
\text { 1995 } \\
\text { 8. Pittard, } 2003 \\
\text { 9. Priestley et al., } 2004\end{array}$ \\
\hline $\begin{array}{l}\text { Recognition and incidence of clinical } \\
\text { instability prior to cardiac arrest/unplanned } \\
\text { intensive care admission }\end{array}$ & $\begin{array}{l}\text { 10. Buist, Jarmelowski, Burton, Bernard, } \\
\text { Waxman \& Anderson, } 1999 \\
\text { 11. Franklin \& Mathew, } 1994 \\
\text { 12. Goldhill, White \& Sumner, } 1999 \\
\text { 13. Hodgetts et al., } 2002 \\
\text { 14. Schein, Hazday, Pena, Ruben, \& } \\
\text { Sprung, } 1990\end{array}$ \\
\hline Shortfalls in care & $\begin{array}{l}\text { 15. McGloin, Adam \& Singer, } 1999 \\
\text { 16. McQuillan et al., } 1998 \\
\text { 17. Seward et al., } 2003\end{array}$ \\
\hline $\begin{array}{l}\text { The experiences of ward nurses caring for } \\
\text { acutely unwell ward patients }\end{array}$ & $\begin{array}{l}\text { 18. Cox, James, \& Hunt, } 2006 \\
\text { 19. Lawless, Wan \& Zeng, } 2010\end{array}$ \\
\hline $\begin{array}{l}\text { Identification of educational requirements } \\
\text { for ward staff }\end{array}$ & $\begin{array}{l}\text { 20. Cutler, } 2002 \\
\text { 21. Derham, } 2007 \\
\text { 22. Smith \& Poplett, } 2002 \\
\text { 23. Wood, Douglas, \& Priest, } 2004\end{array}$ \\
\hline $\begin{array}{l}\text { Causal and contributory factors to } \\
\text { increased mortality }\end{array}$ & $\begin{array}{l}\text { 24. Aiken, Clarke, Sloane, Sochalski \& } \\
\text { Silber } 2002 \\
\text { 25. Rafferty et al., } 2007 \\
\text { 26. Tourangeau et al., } 2006\end{array}$ \\
\hline $\begin{array}{l}\text { Causal and contributory factors to patient } \\
\text { deterioration }\end{array}$ & 27. NPSA, 2007 \\
\hline
\end{tabular}


Table 3 (continued). Articles and reports selected for review to inform the concept analysis

\begin{tabular}{|c|c|}
\hline \multicolumn{2}{|c|}{ Research } \\
\hline Study Focus & Authors \\
\hline $\begin{array}{l}\text { Identification of patients at risk of } \\
\text { deterioration }\end{array}$ & $\begin{array}{l}\text { 28. Buist, Bernard, Nguyen, Moore \& } \\
\text { Anderson, } 2004 \\
\text { 29. Chellel et al., } 2002 \\
\text { 30. Cioffi, } 2000 \\
\text { 31. Endacott, Kidd, Chaboyer \& } \\
\text { Edington, } 2007 \\
\text { 32. Morrice \& Simpson, } 2007 \\
\text { 33. Ryan, Cadman, \& Hann, } 2004 \\
\text { 34. Wheatley, } 2006\end{array}$ \\
\hline $\begin{array}{l}\text { Identification of acutely unwell ward } \\
\text { patients through early warning scores }\end{array}$ & $\begin{array}{l}\text { 35. Andrews \& Waterman, } 2005 \\
\text { 36. Cuthbertson, Boroujerdi, McKie, } \\
\text { Aucott, \& Prescott, } 2007\end{array}$ \\
\hline $\begin{array}{l}\text { Innovative roles to assist in the care of } \\
\text { acutely unwell ward patients }\end{array}$ & $\begin{array}{l}\text { 37. Murch \& Warren, } 2001 \\
\text { 38. Plowright, O'Riordan, \& Scott, } 2005\end{array}$ \\
\hline \multicolumn{2}{|c|}{ Literature reviews } \\
\hline Review Focus & Authors \\
\hline $\begin{array}{l}\text { Cochrane systematic review to determine } \\
\text { the impact of critical care outreach services } \\
\text { on mortality rates }\end{array}$ & 39. McGaughey et al., 2007 \\
\hline $\begin{array}{l}\text { The challenges in caring for acutely unwell } \\
\text { patients }\end{array}$ & $\begin{array}{l}\text { 40. Bion \& Heffner, } 2004 \\
\text { 41. Clarke \& Aiken, } 2003\end{array}$ \\
\hline $\begin{array}{l}\text { Factors influencing suboptimal ward care in } \\
\text { acutely ill patients }\end{array}$ & 42. Massey, Aitken \& Chaboyer, 2008 \\
\hline $\begin{array}{l}\text { Safe care of the acutely unwell ward } \\
\text { patient }\end{array}$ & 43. Bright, Walker, \& Bion, 2003 \\
\hline $\begin{array}{l}\text { Identification of patients at risk of an } \\
\text { adverse event }\end{array}$ & 44. Considine \& Botti, 2004 \\
\hline $\begin{array}{l}\text { The use and evaluation of critical care } \\
\text { outreach services and Medical Emergency } \\
\text { Teams (MET) }\end{array}$ & $\begin{array}{l}\text { 45. Jones, King \& Wilson, } 2009 \\
\text { 46. McArthur-Rouse, } 2001\end{array}$ \\
\hline $\begin{array}{l}\text { The effect of early intervention teams and } \\
\text { early warning scores }\end{array}$ & $\begin{array}{l}\text { 47. Gao et al., } 2007 \\
\text { 48. Reynolds, Cardinal, \& Baxter, } 2005 \\
\text { 49. Robson, } 2002\end{array}$ \\
\hline \multicolumn{2}{|c|}{ Editorials } \\
\hline Editorial Focus & Authors \\
\hline Nurse patient ratios & 50. Clarke \& Aiken, 2006 \\
\hline \multicolumn{2}{|c|}{ Grey Literature } \\
\hline Document Focus & Authors \\
\hline Report on critical incident data & $\begin{array}{l}\text { 51. Thomson, Leuttel, Healey \& Scobie, } \\
2007\end{array}$ \\
\hline Standards of care prior to admission to ICU & 52. NCEPOD, 2005 \\
\hline $\begin{array}{l}\text { Recognition of response to acute illness in } \\
\text { hospital }\end{array}$ & $\begin{array}{l}\text { 53. ACSQH, } 2010 \\
\text { 54. NICE, } 2007\end{array}$ \\
\hline
\end{tabular}


As illustrated in Table 3 the concept of suboptimal care is used frequently in the literature which supports, describes and evaluates specific service delivery initiatives such as Medical Emergency Teams (MET), Critical Care Outreach Services (CCOS), and Early Warning Scores (EWS). The aim of CCOS is to support nurses in the care of acutely unwell ward patients either through direct clinical assistance with patients or through education (Department of Health, 2000). With METs, specific criteria for notification are set based on abnormal patient observations (Lee, Bishop, Hillman, \& Daffum, 1995). By determining whether their patients meet these criteria nurses can decide whether to call the MET to assist in their patients' care.

Early Warning Scores use physiological measurements (usually pulse, blood pressure, respiratory rate, urine output and level of consciousness) to help identify ward patients who are, or who may become, critically ill. Each physiological variable has a points value so that a final score can be calculated. If the total score exceeds a predefined threshold this should trigger action, such as calling for senior clinical advice, critical care outreach or MET assessment (NICE, 2007). These initiatives are based on the assumption that early recognition of deterioration and earlier intervention will improve patient outcome (Gao et al., 2007). Therefore the aim of such service initiatives is to prevent suboptimal care from occurring by supporting ward staff in the recognition and management of acutely unwell ward patients.

\subsubsection{Summary of uses of the concept}

In academic disciplines outside of healthcare the term suboptimal is associated with a set of solutions which are affected by the context in which they occur. A suboptimal solution can be an acceptable pragmatic decision. In a broader healthcare context suboptimal care is care which is less than optimal. Over the past 13 years, the concept of suboptimal care has been frequently used in the context of the acutely unwell ward patient. In the literature, use of the term suboptimal care has usually been in relation to McQuillan et al's. (1998) work, leaving its use, and meaning, vague. What is clear however is that most healthcare authors agree that suboptimal care is either avoidable or preventable. 


\subsection{Defining attributes}

Having identified the uses of the concept of suboptimal care, further clarity and explanation of the concept is now undertaken following the stages in Table 1. The defining attributes of a concept can be described as a list of criteria that if present, differentiate the specific phenomenon from another or similar one (Walker \& Avant, 2005). Identifying the defining attributes is important to this study as these have been identified by investigators as key to the occurrence of suboptimal care. Explicating these attributes will identify contributing factors affecting care of acutely unwell ward patients to inform the design of this study. Within the literature reviewed, recurring themes emerged which can be summarised into three key defining attributes of suboptimal care: poor assessment; delays in diagnosis, treatment or referral; and inadequate or inappropriate patient management.

\subsubsection{Poor Assessment}

Patient assessment is the process of taking, recording, and interpreting clinical data (Wheatley, 2006). Poor assessment or the absence of patient assessment may lead to non recognition of the severity of the patient's condition (Thomson et al., 2007). Accepting that recording the assessment findings is part of the assessment process, the most frequently occurring issue regarding poor assessment includes failure to record patient observations (Endacott et al., 2007; Goldhill, White \& Sumner, 1999; Thomson et al., 2007). Several studies have demonstrated that documentation of physiological data is often missing, recorded intermittently or imprecisely.

A UK retrospective chart review of 76 patients admitted to ICU from the wards found that, despite the patient's severity of illness, some routine physiological observations were missing in the notes of all patients. For example six hours prior to admission to ICU $25 \%, 19 / 76)$ of patients did not have pulse or blood pressure (BP) recorded, 35\% (26/76) did not have respiratory rate recorded, and 51\% (39/76) did not have an assessment of their level of consciousness (Goldhill, White, \& Sumner, 1999). 
Several years later and despite the introduction of EWS, two studies found similar results. Endacott et al. (2007) audited 17 sets of charts and progress notes of patients unexpectedly admitted to ICU as part of an Australian case study which aimed to identify how nurses and doctors recognise and communicate patient deterioration. These authors noted that none of the observation charts contained complete documentation of patient vital signs. Vital signs most frequently omitted were respiratory rate $(9 / 17$ charts) and temperature (10/17). Although this audit was of only 17 sets of patient notes, similar results were illustrated in a report of 64 critical incidents. Similarly Thomson et al., (2007) demonstrated that in 14 (22\%) incidents no observations were recorded for a prolonged period prior to the patient's death. Furthermore in 30 incidents $(47 \%)$ observations were recorded but deterioration was not recognised. Why healthcare staff fail to recognise clinical deterioration is not addressed within these studies.

\subsubsection{Delays in diagnosis, seeking advice, treatment or referral}

Within this overall theme the most common issue is the presence of physiological deterioration which either is not recognised or is not acted upon. Two studies carried out in the USA used retrospective chart review by assessors to determine the frequency and characteristics of physiological deterioration prior to cardiac arrest (Franklin \& Mathew, 1994; Schein et al, 1990). As discussed in section 2.4.3 these studies identified that between 60\% (99/155) and 84\% (54/64) of patients had physiological deterioration which, although documented, was not acted upon within six to eight hours of cardiac arrest occurring. Although patient deterioration was documented by nursing staff there were delays in informing medical staff. When medical staff were informed by nurses of patient deterioration such information was perceived by doctors as either unimportant or unreliable (Franklin \& Mathew, 1994; Schein et al.,1990). Why medical staff had this perception is not explored.

These studies, although 20 years old, are frequently cited, yet delays in the management of acutely unwell patients are still frequently reported. One more recent UK retrospective chart review which studied 139 case notes of patients who had suffered a cardiac arrest found that in $35 \%(23 / 78)$ of patients there were 
delays by nursing staff in informing a doctor of the patient's deterioration; delays in doctors responding to nurses' request to attend and delays in diagnosis (Hodgetts et al., 2002).

Delays in seeking advice were also demonstrated in McQuillan et al's. (1998) work. In $15 \%$ of cases (8/54 patients) failure to seek advice was the cause of suboptimal care. Likewise delayed referral to specialty areas led to suboptimal care in $42 \%$ of cases (41/99 patients), when junior doctors failed to inform the ICU triage officer of acutely unwell patients (Franklin \& Mathew, 1994).

A further defining attribute of suboptimal care, that of delayed review by a senior doctor, has also been demonstrated (NCEPOD, 2005). In this UK National Confidential Enquiry, of the 439 case notes reviewed only $58 \%$ of patients saw a consultant physician within 24 hours of admission. Furthermore, although an acceptable patient history and examination had been conducted, initial treatment was often delayed. It could be hypothesised that junior doctors delayed initiating their treatment plan until it was endorsed by a senior member of the team, causing a delay which led to suboptimal care.

A recent UK-based report examined 64 critical incident reports which occurred on general surgical or medical wards where patient deterioration was either not recognised or not acted upon (Thomson et al., 2007). This report noted that whilst patient deterioration was recognised and medical attention sought, delays in the patient receiving medical attention occurred in 17 cases. This deterioration was often recognised by nursing staff who were unable to get medical help quickly (Thomson et al., 2007). Whilst these authors do not provide explanation for this, it could be argued that this is further demonstration of medical staff not perceiving information supplied by nursing staff to be reliable or important.

\subsubsection{Inappropriate or inadequate patient management}

In Seward et al's (2003) UK study inadequate patient management related to insufficient oxygen and fluid management, insufficient treatment of hyperkalemia, and failure to request blood glucose measurement in $86 \%(172 / 200)$ of patients. These findings are very similar to McQuillan et al's (1998) results in which "failure to appreciate that airway, breathing and circulation are the prerequisites of life and 
that their dysfunction are the common denominators of death" (p. 1859) were demonstrated. Finally, inadequate patient management includes inadequate patient stabilization prior to transfer to ICU (Franklin \& Mathew, 1994).

In summary, there are multiple defining attributes of suboptimal care of the acutely unwell ward patient. These are poor assessment of patients, delays in diagnosis, seeking advice, treatment or referrals, and inappropriate or inadequate patient management. Any one or a combination of these attributes may lead to suboptimal care. Exploring the defining attributes has been useful, as it has prompted further enquiry. Within these studies the defining attributes of suboptimal care are clear but there is limited exploration or explanation for why these attributes occur. Exploring the antecedents of suboptimal care should provide some clarification.

\subsection{Antecedents and Consequences of suboptimal care}

Antecedents are those events and incidents (pre-disposing factors) that must occur prior to the occurrence of the concept (Walker \& Avant, 2005). For this study a thorough exploration of the antecedents to suboptimal care is required as these are the factors affecting care delivery to acutely unwell patients. The literature revealed several predisposing factors which can be organised into four distinct domains: patient complexity; healthcare workforce; education; and organisation. Figure 1 demonstrates the antecedents of suboptimal care, the defining attributes and suboptimal care that arise from this concept analysis. 
Antecedents

Patient Complexity

Healthcare

Workforce

Education

Organisation

\section{Defining Attributes}

Poor Assessment

Delays in diagnosis, treatment or referral

Inappropriate or inadequate management
Suboptimal

Care

\section{Figure 1. Antecedents and defining attributes of suboptimal care}

\subsubsection{Patient Complexity}

There is no doubt that ward patient profiles have become more complex, leading to increased patient acuity and nurse workload. Explanations for this include an aging population, an increase in healthcare technology, differences in the way healthcare is provided (e.g. more day case surgery), and an increase in patients with multiple co-morbidities (Bion \& Heffner, 2004; Bright et al., 2004). It could be suggested that a 'straightforward' ward patient no longer exists. The potential for deterioration is therefore greater. Furthermore the numbers of critically ill patients nursed in ward areas are high. In one UK survey of 82 wards in four National Health Service Trusts (NHS), 12\% ( $n=1873$ ) of ward patients required more care than would normally be available at ward level (Chellel et al., 2002).

\subsubsection{Health Care Workforce}

Healthcare workforce was a second domain identified as a key antecedent in this concept analysis of suboptimal care. This domain is multi factorial with workload, nurse patient ratios and skill mix alterations, and changes in working practices impacting on team working. 


\subsubsection{Workload, nurse patient ratios and skill mix}

A key facet of the nurses' role has been described as surveillance. Surveillance involves frequent assessment of patients, picking up on cues, and then recognizing complications (Clarke \& Aitken, 2003). However, nurses are unable to apply their knowledge and skills to seriously unwell patients on the wards due to increased workload and inadequate nurse to patient ratios (Cutler, 2002). The need for adequate nurse to patient ratios is well described in the literature (Aiken et al., 2002; Clarke \& Aiken, 2006; Lawless, Wan, \& Zeng, 2010; Rafferty et al., 2007; Torangeau et al., 2007) with detrimental outcomes including increased mortality and increased length of stay demonstrated with inadequate ratios. Further, nurses are frequently interrupted by phone calls, doctors' requests, attending to the needs of other patients and relatives and accompanying patients to other departments (NPSA, 2007). Strategies employed by nurses to cope with heavy workloads include working beyond the end of their shift, going without breaks and 'rationing care' according to a recent New Zealand survey of nurses working in three District Health Boards (DHB) (Lawless et al., 2010). In this survey one aspect of patient care reported as rationed by respondents was monitoring the patient's condition. Therefore with too many patients to care for, nurses are unable to adequately provide this surveillance and/or subsequently process the information they have obtained. Further demands on nurses' time include having meal breaks, teaching and supporting newly qualified staff or attending doctors' ward rounds (NPSA, 2007). Inadequate nurse-patient ratios will have a negative impact on the overall quality of patient assessment, which may lead to suboptimal care.

However, nurse patient ratios should not be viewed in isolation. The collective skills, experience, and clinical knowledge of the nursing team should also be taken into account (Endacott et al., 2007). Nevertheless even the most experienced nurse will struggle to manage with a heavy patient load (Cioffi, 2000) as experienced nurses will also be used by more novice nurses for support and advice. Where there is a lack of experienced nurses and subsequent poor skill mix care can become task focused and patient deterioration may go unnoticed (Endacott et al., 2007; NPSA, 2007). 


\subsubsection{Changes in working practices}

Changes in the training and working practices of doctors may also contribute to the development of suboptimal care. Within the European Union (EU) the implementation of the Working Time Directive $(\mathrm{DOH}, 2009)$ has reduced the average working week for a trainee doctor to 48 hours per week in Europe. To provide adequate medical cover shift work has been introduced, leading to multiple patient handovers within teams, which in turn leads to discontinuities of care and communication failures (Bion \& Heffner, 2004). The combination of frequent rotations through different clinical areas and a reduction in junior doctors' hours may result in reduced exposure to clinical problems and clinical education (NCEPOD, 2005). The inability to recognise an acutely unwell patient may be attributable to a lack of clinical exposure to this patient group. Furthermore, heavy workloads are an issue for junior doctors, especially after hours when staffing is minimal. According to interview data from a mixed methods study carried out in the UK by the NPSA (2007), prioritisation of which calls to attend to first is an issue for junior medical staff at weekends and overnight. This is especially relevant when communication of the patient condition does not convey the urgency required.

Whilst none of the research reviewed for this section of the analysis was conducted in New Zealand it is important to note that medical staff in New Zealand experience similar working practices. According to the latest New Zealand Resident Doctors Association (RDA) agreement junior doctors can only work a maximum of 60 hours per week furthermore they should not be required to work more than 72 hours in any consecutive seven days nor more than 16 hours in any day (NZRDA, 2011). Shift work for New Zealand doctors is also the norm.

\subsubsection{Communication and Team working}

A key defining attribute of suboptimal care is delays in treatment, diagnosis, or referral. This has been attributed by one study to poor verbal and written communication (NCEPOD, 2005). This is supported by a UK exploratory, descriptive study of seven nurses on a medical ward which demonstrated that nurses who have concerns about patients have difficulty in 'getting action' for them, which may lead 
to delays (Cox et al., 2006). Furthermore two studies have demonstrated that the ability to communicate deterioration effectively is dependent upon knowledge, confidence and level of experience (Andrews \& Waterman, 2005; Wood et al., 2004). When these are lacking, patient care can be suboptimal.

According to a mixed methods study undertaken in the UK, patient documentation is often incomplete, missing, or difficult to read (NPSA, 2007). This study used interviews, focus groups, and root cause analysis (RCA) to identify the underlying causal and contributory factors to deterioration incidents. The findings from this study identified incomplete and inadequate written information as impacting on the care of deteriorating patients.

As a result of these studies, guidelines relating to explicit plans of care have been developed. The National Institute of Health and Clinical Excellence (NICE) guideline 50 (2007) recommends that all patients admitted to hospital should have a monitoring plan written in the notes on admission/initial assessment. This should specify the type and frequency of the physiological observations to be recorded. This is especially relevant when patients are being transferred between ward areas. Patients are at risk of suboptimal care when transferred back to the ward area from a critical care environment due to poor communication between the two areas (NICE, 2007). Verbal and written plans from both the nursing and medical staff have been recommended. Ensuring that this plan is discussed with the receiving ward to ensure that they have the necessary resources and ability to deliver the plan is recommended (NICE, 2007).

In the absence of an effective teamwork culture there is likely to be a higher number of problems related to suboptimal care (NPSA, 2007). Shift work, staff shortages, over reliance on casual, locum and agency staff, and poorly structured medical teams all have detrimental effects on teamwork (Endacott et al., 2007; NCEPOD, 2005). Frequent changes of staff lead to team members not getting to know each other or build professional trust. This can result in a lack of clarity of roles within the team, professional hierarchies and lack of support within the team (NPSA, 2007). When professional hierarchies exist, junior staff are reluctant to call for help when they are in difficulty for fear of 'losing face' or being admonished by 
senior team members. Furthermore, multiple specialty involvement in a patient's care is also complicated. Team working between specialties and determining who takes overall responsibility for that patient is difficult, leading to delays in patient management (NPSA, 2007).

\subsubsection{Education}

There has been increased emphasis on the educational needs of staff caring for acutely unwell patients (DoH, 2000; NCEPOD, 2005; NPSA, 2007). This has been a response to studies identifying knowledge and skill deficits that have led to suboptimal care (McGloin et al., 1999; McQuillan, et al., 1998). These knowledge and skill deficits are related to patient assessment skills as well as education and training about managing acutely unwell or deteriorating patients.

\subsubsection{Assessment skills}

Lack of recognition of deterioration is a defining attribute of suboptimal care. This has been blamed on poor assessment skills in both nursing and medical staff (McGloin et al., 1999; McQuillan et al., 1998). Failure to appreciate clinical urgency is also related to the ability to determine which vital signs should be acted upon immediately (Buist et al., 1999; Franklin \& Mathew, 1994; Hodgetts et al., 2002; Schein et al., 1990). Additional research by Smith and Poplett (2002) demonstrated significant knowledge gaps in preregistration house officers (PRHO) and senior house officers (SHO). In this study PRHOs and SHOs lacked knowledge and understanding in interpreting the signs of acute illness (pulse oximetry, capillary refill time, oxygen therapy and the management of an unconscious patient). Carrying out an appropriate assessment and determining the best course of action is related to both clinical exposure and appropriate education and training.

\subsubsection{Education and Training}

Much of the literature reviewed suggests that a lack of education and training are to blame for suboptimal care and recommends that staff are educated in the key elements of managing acutely unwell ward patients (for example Bright et al., 2004; Franklin \& Mathew, 1994; McGloin et al., 1999; McQuillan et al., 1998; 
NCEPOD, 2005; Wood et al., 2004). Recommendations range from the inclusion of assessment and management of acutely unwell ward patients in undergraduate nursing and medical curricula (Buist et al., 1999; Endacott et al., 2007; Wood et al., 2004), rotation of staff through critical care areas (Andrews \& Waterman, 2005; McQuillan et al., 1998), and interdisciplinary courses which are physiology-based such as ALERT ${ }^{\mathrm{TM}}$ (Andrews \& Waterman, 2005). This emphasis on education and training is based on the assumption that a better educated workforce leads to better cared for patients. However this relies upon what Cutler (2002) describes as 'practical applicability' (p. 283); this is, the ability to implement knowledge and skills within a context.

\subsubsection{Organisation}

Organisational factors have been demonstrated as antecedents to suboptimal care. These are related to the way in which patient care is organised and the availability of equipment. Firstly, how medical care is organised is a predisposing factor which contributes to these delays in seeking advice. Often the most junior doctors are seeing the sickest patients with little support from more senior doctors. McQuillan et al., (1998) noted lack of supervision for junior doctors in 1998. This was noted by NCEPOD, in 2005, and again by NPSA in 2007, demonstrating the ongoing nature of this issue. Organisational processes which continue to support the 'on call system' whereby patient care is reliant upon a junior doctor 'referring up' is problematic. In a UK review of case notes from $118(\mathrm{~N}=139)$ consecutive inhospital cardiac arrests, medical review was limited to a PRHO in $45 \%$ of cases (Hodgetts et al., 2002). Registrars are often busy in clinics or the emergency department and after hours, consultants are at home. Junior doctors are reluctant to increase the registrar's workload and fear judgment by consultants of their ability to cope (NPSA, 2007). The use of evidence-based guidelines to assist junior medical staff in the care of acutely unwell ward patients is one potential solution (McQuillan et al., 1998), although it has been shown guidelines or protocols are not readily adhered to in busy environments (NPSA, 2007).

How nursing care is organised has also changed. Fiscal constraints and the reality of a global nursing shortage have led to the greater utilisation of Health Care 
Assistants (HCA) within the UK and USA workforce. HCAs are support workers who work in clinical areas under the direct supervision of a registered nurse (RN). These support workers are now often the main source of direct patient care within some NHS Trusts in the UK (NPSA, 2007). This has been shown to be an issue as HCAs may receive training in some complex aspects of patient care such as taking observations, but may lack the knowledge to both recognise abnormality and act appropriately (NPSA, 2007). Registered nurses' capacity to supervise HCAs in addition to their other responsibilities discussed earlier increases an already heavy workload. Consequently, patient deterioration may go undetected.

The time and place of admission for patients may also lead to suboptimal care. According to one UK retrospective chart review, patients admitted between the hours of midnight and 9 am were more likely to die unexpectedly (Seward et al., 2003). Furthermore, infrequent observations (heart rate and blood pressure) during the night may be a compounding factor. Two studies (one Australian and one UK) carried out interviews with ward staff and found that staff rarely carry out observations overnight (Endacott et al., 2007; NPSA, 2007).

Admission to inappropriate wards (a medical patient nursed in a surgical area or vice versa) may also result in suboptimal care (Hodgetts et al., 2002). Hodgetts et al's retrospective chart review of 118 adult in-hospital cardiac arrests demonstrated that the odds of potentially avoidable cardiac arrest was 12.6 times greater for patients nursed in a clinical area believed to be inappropriate for their main complaint. According to these authors, $20(n=118)$ cardiac arrests occurred in patients who were nursed in an inappropriate area. This included patients being nursed on a general ward when they should have been in a critical care area. It also included six acute medical patients who were nursed on surgical wards. These "outliers" or "borders" as they are often termed, present a challenge to nursing staff who may not be familiar with either the specialty required care, or specific documentation for that specialty (NPSA, 2007). As a result, patients' specific needs may go unmet and the potential for deterioration increases. 


\subsubsection{Adequate resources/over reliance on equipment}

The availability, reliability and consistency in equipment between areas has been shown to contribute to the delay in care for some patients (Wood et al., 2004). Difficulties in obtaining equipment or unfamiliarity with equipment owing to the number of different devices within hospitals have been reported (Cox et al., 2006). Furthermore relying solely upon task-oriented vital signs assessment as recorded by machines has risk implications. Important clinical information ascertained by a full patient assessment utilising sensory skills is likely to be missed (Wheatley, 2006).

\subsection{Consequences of suboptimal care}

In the model of concept analysis proposed by Walker \& Avant (2005) consequences follow an occurrence of the concept. The consequences of suboptimal care have been identified as critical events such as unexpected death, unexpected admission to ICU and cardiac arrest. Investigators have used these specific end points as a means of identifying patients for inclusion in studies looking at the prevalence and incidence of suboptimal care (Buist et al., 1999; Franklin \& Mathew, 1994; Goldhill et al., 1999; Schein et al., 1990). Exploring the consequences of suboptimal care enables quantification of the number of patients affected to date and reveals the extent of the problem, thus providing further impetus for this study. Table 4 illustrates the number of patients suffering consequences related to suboptimal care.

Table 4. Number of patients suffering consequences related to suboptimal care

\begin{tabular}{|c|c|c|c|}
\hline Category & Authors & Study design and aim & $\begin{array}{l}\text { Proportion of } \\
\text { patients } \\
\text { suffering } \\
\text { consequences } \\
(\%)\end{array}$ \\
\hline \multirow[t]{2}{*}{ Cardiac arrest } & $\begin{array}{l}\text { Schein et } \\
\text { al. (1990) }\end{array}$ & $\begin{array}{l}\text { Retrospective chart review to } \\
\text { study physiologic abnormalities } \\
\text { prior to cardiac arrest }\end{array}$ & $54 / 64(84 \%)$ \\
\hline & $\begin{array}{l}\text { Franklin } \\
\text { and } \\
\text { Mathew, } \\
\text { (1994) }\end{array}$ & $\begin{array}{l}\text { Retrospective chart review to } \\
\text { determine the frequency of } \\
\text { premonitory signs and symptoms } \\
\text { prior to cardiac arrest in patients } \\
\text { on general medical wards }\end{array}$ & $99 / 150(66 \%)$ \\
\hline
\end{tabular}


Table 4 (continued). Number of patients suffering consequences related to suboptimal care

\begin{tabular}{|c|c|c|c|}
\hline Category & Authors & Study design and aim & $\begin{array}{l}\text { Proportion of } \\
\text { patients } \\
\text { suffering } \\
\text { consequences } \\
(\%)\end{array}$ \\
\hline & $\begin{array}{l}\text { Buist et al. } \\
\text { (1999) }\end{array}$ & $\begin{array}{l}\text { Retrospective chart review to } \\
\text { investigate the nature and } \\
\text { duration of clinical instability in } \\
\text { hospital patients before a critical } \\
\text { event (cardiac arrest/unplanned } \\
\text { admission to ICU) }\end{array}$ & $37 / 112(33 \%)$ \\
\hline & $\begin{array}{l}\text { Hodgetts et } \\
\text { al. (2002) }\end{array}$ & $\begin{array}{l}\text { Retrospective chart review to } \\
\text { determine the number of } \\
\text { avoidable cardiac arrests and } \\
\text { whether location, individual or } \\
\text { system factors may influence } \\
\text { these }\end{array}$ & $73 / 118(62 \%)$ \\
\hline \multirow[t]{3}{*}{$\begin{array}{l}\text { Unanticipated } \\
\text { admission to } \\
\text { ICU from the } \\
\text { wards }\end{array}$} & $\begin{array}{l}\text { Goldhill, } \\
\text { White, and } \\
\text { Sumner } \\
\text { (1999) }\end{array}$ & $\begin{array}{l}\text { Retrospective chart review to } \\
\text { describe the reasons for ICU } \\
\text { admission in in-hospital patients } \\
\text { and identify physiological values } \\
\text { and interventions associated with } \\
\text { patients at risk }\end{array}$ & $76 / 168$ (45\%) \\
\hline & $\begin{array}{l}\text { McGloin et } \\
\text { al. (1999) }\end{array}$ & $\begin{array}{l}\text { Retrospective chart audit to } \\
\text { explore unexpected deaths } \\
\text { occurring on wards and whether } \\
\text { they were preventable and } \\
\text { unexpected ICU admissions in } \\
\text { terms of quality of care }\end{array}$ & $31 / 86(36 \%)$ \\
\hline & $\begin{array}{l}\text { Buist et al. } \\
\text { (1999) }\end{array}$ & $\begin{array}{l}\text { Retrospective chart review to } \\
\text { investigate the nature and } \\
\text { duration of clinical instability in } \\
\text { hospital patients before a critical } \\
\text { event (cardiac arrest/unplanned } \\
\text { admission to ICU) }\end{array}$ & 79/112 (70\%) \\
\hline $\begin{array}{l}\text { Unexpected } \\
\text { deaths }\end{array}$ & $\begin{array}{l}\text { McGloin et } \\
\text { al. (1999) }\end{array}$ & $\begin{array}{l}\text { Retrospective chart audit to } \\
\text { explore unexpected deaths } \\
\text { occurring on wards and whether } \\
\text { they were preventable and } \\
\text { unexpected ICU admissions in } \\
\text { terms of quality of care }\end{array}$ & $13 / 317(4 \%)$ \\
\hline
\end{tabular}


Table 4 (continued). Number of patients suffering consequences related to suboptimal care

\begin{tabular}{|c|c|c|c|}
\hline Category & Authors & Study design and aim & $\begin{array}{l}\text { Proportion of } \\
\text { patients } \\
\text { suffering } \\
\text { consequences } \\
(\%)\end{array}$ \\
\hline & $\begin{array}{l}\text { Seward et } \\
\text { al. (2003) }\end{array}$ & $\begin{array}{l}\text { Retrospective chart review to } \\
\text { determine feasibility of } \\
\text { conducting a confidential enquiry } \\
\text { into why patients die after } \\
\text { emergency admission to hospital }\end{array}$ & $\begin{array}{l}14-25 / 200 \\
(7 \%-12 \%) \\
\text { * Range reflects } \\
\text { agreement } \\
\text { between } \\
\text { assessors }\end{array}$ \\
\hline & $\begin{array}{l}\text { Thomson } \\
\text { et al. } \\
\text { (2007) }\end{array}$ & $\begin{array}{l}\text { Retrospective review of clinical } \\
\text { incident data to examine the } \\
\text { deaths of inpatients in one year } \\
\text { reported to the National } \\
\text { Reporting and Learning System }\end{array}$ & $64 / 425$ (15\%) \\
\hline
\end{tabular}

Table 4 demonstrates that the percentage of patients suffering consequences of suboptimal care varies depending on the type of consequence. The range of patients who suffered a cardiac arrest in the studies reviewed ranged from $33 \%$ to $84 \%$. When patients suffer cardiac arrests within general wards these are not always sudden events. As discussed earlier, studies suggest that hospital cardiac arrests are preceded by at least one abnormal clinical observation which is either not recognised or acted upon (Franklin \& Mathew, 1994; Schein et al., 1990).

The percentage of patients whose admission to ICU was unexpected ranged from $36 \%$ to $70 \%$. In addition mortality rates in this group of patients were significantly higher than in the general hospital population. In one study of patients who were deemed to have had suboptimal care, mortality was $65 \%$ in comparison to $42 \%$ in the better-managed group ( $p<0.0001$ ), (McGloin, et al., 1999).

Finally the percentage ranged from $4 \%$ to $15 \%$ in patients whose death was unexpected, but believed to be a consequence of suboptimal care. In one study carried out over six months, preventing suboptimal care equated to "two potentially avoidable ward deaths each month" (McGloin, et al., 1999, p.258). 
Trying to eliminate suboptimal care entirely may be an unattainable goal but implementing strategies aimed at reducing the number of patients it affects should be possible. Identifying factors which lead to suboptimal care through conducting this study may inform strategy development.

\subsection{Identifying a model case}

The next stage of conducting a concept analysis according to Walker and Avant (2005) is to construct a model case. Walker and Avant state that a "model case is an example of the concept that demonstrates all the defining attributes of the concept" (p.69). Given that Walker and Avant's approach to concept analysis is iterative, the model case can be created at any stage of the concept analysis. They suggest that the model case's development can come first, may be developed simultaneously with the defining attributes, or may emerge after the development of the defining attributes. Walker and Avant also suggest that a process of 'internal dialogue' through creating cases is crucial to test the defining attributes to determine which 'fit' best with the concept. Whatever approach is taken, Walker and Avant advise that the model case should be set up in a way that is useful to the analysis.

For this concept analysis the model case was constructed after clarifying both the defining attributes and the antecedents of suboptimal care. This allowed all aspects of suboptimal care to be combined within one scenario, which then could demonstrate the relationship between the antecedents and the defining attributes. The following constructed model case clearly demonstrates the defining attributes of suboptimal care (delays in diagnosis, poor assessment, and inadequate patient management) and some antecedents (poor staffing levels, inadequate communication) detailed previously.

\subsubsection{Model Case.}

Mrs. Smith, a 67-year-old lady with type 2 diabetes, is admitted with suspected pneumonia on a Friday evening at 1900 to a surgical ward because there are no medical beds. The surgical ward is short of two members of staff due to sickness. There are 3 staff (2 nurses, one HCA) for 27 patients. The ward has also received two patients transferred from the Intensive Care Unit. The emergency 
department (ED) nurse on handover to the allocated ward nurse notes that Mrs. Smith is on 4 litres of Oxygen via a Hudson face mask, is tachypnoiec with a rate of 34 breaths per minute, oxygen saturations are $95 \%$, she is pyrexial $38.7^{\circ} \mathrm{C}$, hypotensive $89 / 55 \mathrm{MMhG}$, tachycardic $110 \mathrm{bpm}$, blood sugar is $7.3 \mathrm{mmol} / \mathrm{L}$ and she has not passed urine since entering the ED. She has had a Chest $X$-ray $(C X R)$ in ED but it has not yet been reviewed. She is receiving IV normal saline at $100 \mathrm{~m} / \mathrm{s} \mathrm{hr}$ which is due to run through within an hour. The admitting nurse contacts the house surgeon on call to tell him that Mrs. Smith has arrived on the ward and needs more fluids prescribing. She does not tell him about Mrs. Smith's abnormal vital signs. Three hours later the house surgeon comes to see Mrs. Smith. She has not had any observations taken since her admission. When the house surgeon examines her he notes that her respiratory rate is $48 \mathrm{bpm}$, saturations are $88 \%$ and that she appears drowsy. He tries to take an arterial blood gas but is unsuccessful. He asks the nurse to increase the oxygen to $61 / \mathrm{min}$ and leaves the ward to see another patient without reviewing the CXR. At 2315 when the night staff come on Mrs. Smith is noted to be unresponsive and a cardiac arrest call is made. Mrs. Smith is successfully resuscitated, transferred to ICU but dies two days later.

\subsection{Identifying additional cases}

Identifying additional cases is part of the process of internal dialogue referred to earlier (Walker \& Avant, 2005). Walker and Avant believe that examining similar or contrary cases to the concept of interest will enable the choice of the defining attributes which have the best fit. They suggest that borderline, related, invented, and contrary cases should be used. These can be constructed, real-life examples or can be taken from the literature (Walker \& Avant).

Walker and Avant (2005) state that "contrary cases are clear examples of not the concept" (p.71). In this constructed contrary case, there is clear evidence of prompt referral and treatment, accurate assessment and appropriate management.

Patient noted to be hypotensive, tachycardic and tachypnoeic by nurse. Doctor called and reviewed patient immediately. Patient thoroughly assessed by registrar. Fluid challenges given, oxygen therapy prescribed and hourly observations ordered. Patient reviewed by doctor within one hour to assess impact of therapy.

Borderline cases contain most of the defining attributes of the concept but not all of them, whereas related cases demonstrate ideas that are very similar to the concept under examination but which, when scrutinized, are different (Walker \& 
Avant, 2005). For this concept analysis creating borderline and related cases was problematic. Suboptimal care is a recent phenomenon in health care, and there is minimal research to inform this stage of concept analysis. It also seemed unnecessary at this stage to try to construct cases illustrating what was similar to or nearly suboptimal care, given the clarity which emerged during the construction of the model case.

\subsection{Empirical Referents}

The final step in Walker and Avant's (2005) model of concept analysis is to determine the empirical referents for the defining attributes. Empirical referents of a concept are "classes or categories of actual phenomena that by their existence or presence demonstrate the occurrence of the concept itself" (Walker \& Avant, 2005, p. 73). Delays in diagnosis, treatment, or referral, and poor assessment and inadequate or inappropriate patient management have been identified in this concept analysis as defining attributes of suboptimal care. An example of an empirical referent in this instance would be length of time taken by ward staff to notify the Critical Care Outreach team or Medical Emergency Team of a patient with a high early warning score. A retrospective research design would be required to capture these attributes, meaning that suboptimal care will have already occurred. However, continuing to investigate this aspect of suboptimal care informs us only of the extent of the problem. It does little to explain why suboptimal care has occurred. Rather than focus on the defining attributes of suboptimal care investigators should focus on the antecedents to suboptimal care in order to prevent the occurrence of suboptimal care. In summary, strictly adhering to Walker and Avant's model would lead investigators to concentrate on developing research measures which would provide evidence of the extent of suboptimal care. What are required are objective measures that capture which factors affect care in this context.

\subsection{Methodological limitations of reviewed studies}

The research reviewed for this concept analysis can be categorized into two main research design groups. These are quantitative descriptive designs and qualitative exploratory designs. A large proportion of the research reviewed for 
this concept analysis was quantitative in methodology, with retrospective chart review being the predominant research design (Buist et al., 1999; Franklin \& Mathew, 1994; Goldhill, White \& Sumner, 1999; Hodgetts et al., 2002; McGloin, Adam \& Singer, 1999; McQuillan et al., 1998; NCEPOD, 2005; Seward et al., 2003; Schein et al., 1990; Thomson et al., 2007). Results from retrospective chart reviews may suffer from observational bias ${ }^{7}$. Furthermore the quality of information recorded by healthcare professionals is variable and adds to the list of limitations of using documentation which was not originally intended for research purposes. Documentation is often missing, there may be disagreement in interpretation of information recorded in charts (including inconsistent use of jargon and acronyms) and finally authentication of information and difficulty establishing cause and effect is challenging (Gearing, Mian, Barber, \& Ickowicz, 2006; Hess, 2004).

Some authors of the studies reviewed which used retrospective chart review have attempted to reduce the effects of these limitations through restricting the type of data being collected to objective, easily identifiable data (Hodgetts et al, 2003; Seward et al, 1999) and by blinding reviewers to initial diagnosis and patient outcome (McGloin, et al., 1999). However it is important to note that given the aims of some of these studies (i.e. to determine the prevalence and incidence of suboptimal care), the use of prospective research designs is virtually impossible.

The second group of studies reviewed for this concept analysis use qualitative exploratory methodologies, with interviews and focus groups being the main research tools for data collection (Chellel, Higgs, \& Scholes, 2006; Cioffi, 2000; Cox, James, \& Hunt, 2006; Cutler, 2002). The nature of exploratory designs makes generalising any results to other health care settings problematic, therefore limiting the applicability of this type of research. Highlighting the limitations of each of these designs is important in order to demonstrate rigorous appraisal of the literature reviewed. Whilst acknowledging the limitations of these studies there remains strong evidence indicating that suboptimal care exists.

\footnotetext{
7 "An effect causing unwanted variations in data recorded which are produced because of the characteristics of the observer" (Coolican, 1999, p. 112).
} 


\subsection{Conclusion}

This chapter has explored suboptimal care and its association with the care of the acutely unwell ward patient. Using Walker and Avant's (2005) approach to concept analysis has provided a systematic method of critiquing literature which examines the care of acutely unwell ward patients who receive suboptimal care. A limitation to this concept analysis process was that suboptimal care is a relatively new and underexplored concept. As such, this 'newness' did not allow for construction of some the additional cases as suggested by Walker and Avant.

It is clear that suboptimal care, in the context of the acutely unwell ward patient, has received some attention. However performing this concept analysis has added to what is known about suboptimal care through identifying the complex and multifaceted factors that underpin care of acutely unwell ward patients. Whilst the definition of suboptimal care as offered by McGloin et al. (1999) is supported, this analysis has more importantly explicated suboptimal care in the acute setting. This concept analysis has demonstrated that until now the research lens has been mainly directed to quantification of and solutions to suboptimal care, as opposed to causative factors.

It would therefore appear logical to focus future research on the antecedents to suboptimal care, as these factors may directly affect the degree to which suboptimal care occurs. Through conducting this concept analysis four antecedents have emerged to help understanding and definition of this concept. These antecedents are patient complexity, healthcare workforce, education, and organisation and are the key factors associated with care of the acutely unwell ward patient within the empirical and grey literature. These can now underpin the design of this study and form the foundations of the analytical framework for data analysis. 


\section{CHAPTER 3 - JUSTIFICATION OF CASE STUDY APPROACH AND RESEARCH DESIGN}

\subsection{Introduction}

The concept analysis conducted in Chapter 2 has demonstrated that until now the research lens has been mainly directed to quantification of and solutions to suboptimal care, as opposed to causative factors. However the concept analysis has also identified multiple factors as antecedents to the suboptimal care of acutely unwell ward patients. In order to capture the complexity associated with organisational factors affecting care of acutely unwell ward patients and to determine whether these are influenced by context, a multiple case study approach was chosen for this study.

This chapter presents and justifies the case study methodology used for this study. That is followed by selection of cases and participants, and procedures used in the collection and analysis of data. Finally, strategies used to maintain methodological rigour are presented.

\subsection{Case study methodology}

The aim of this study was to determine which factors affect the care of acutely unwell ward patients from an organisational perspective. Two research objectives have been developed. These are:

- To determine whether factors affecting care of the acutely unwell ward patient are unique to specific contexts

- To identify whether multiple perspectives exist within an organisation regarding factors affecting the care of acutely unwell ward patients.

Caring for acutely unwell ward patients within a ward environment involves many different healthcare staff and care delivery is subject to organisational policies and processes devised and endorsed by managers at ward, senior, and executive level. These organisational policies and processes may affect the ability of nurses and doctors to deliver care. As a result, the context in which the care is delivered may assist or impede care delivery to acutely unwell ward patients. 
Examining the context consequently becomes an important part of the research process. Therefore selection of a research approach which allowed an in-depth exploration of the context and used multiple sources of information to provide a thorough examination of the organisational and ward factors was required.

A case study approach was chosen for three reasons. Firstly, case study research is an in-depth investigation of a "real life phenomenon which takes place within its own context" (Yin, 2003, p. 13). It is used when the researcher wants to deliberately explore the contextual conditions because they are relevant to the phenomenon being studied or because the boundaries between the context and the phenomenon under investigation are not clear (Baxter \& Jack, 2008; Yin, 2003) Unlike experimental research, where the phenomenon under investigation is deliberately removed from the context so that a limited number of variables can be controlled (Yin), case study research seeks to "appreciate its embeddedness and interaction with its context" (Stake, 1995, p.16).

Secondly, case study research promotes the use of multiple sources of data in order to achieve an in-depth examination of that context (Gangeness \& Yurkovich, 2006). Case studies allow the researcher to build a rich description of the cases being studied by using multiple sources of information. Yin (2003) identifies six sources of information: interviews, direct observations, archival records, participant observation and physical artefacts. Using quotations from key participants, excerpts from field notes and using reviewed documentation brings to life the complexity of the many variables inherent in the phenomenon being studied (Hancock \& Algozzine, 2006).

Thirdly, it allows the researcher to explore broad, complex research questions holistically, accepting that there may be many variables which may have an impact on the case being studied (Platt, 1992; Stoecker, 1991). The benefits of using a case study methodology for this study included exploring a range of participants' experiences (nurses, doctors and managers from ward to executive level) regarding the factors affecting the care of acutely unwell ward patients; the opportunity to study all the cases in relation to their context (the hospital the ward was located in); 
and the ability to use many sources of information (interviews, focus groups and document review) to address the research question.

The aim of a case study research is therefore to provide a three dimensional view of the situation where relationships, organisational issues and particular patterns of interest are illustrated (Yin, 2003). As such, it is particularly suitable for this study as it allows the identification of organisational and contextual enablers and barriers; and it describes the circumstances in which acutely unwell patients are cared for.

\subsection{The cases}

Miles and Huberman (1994) suggest that the case is the "heart of the study"; therefore the initial step in case study research is defining what constitutes 'the case'. According to Yin (2003) the selection of what is 'the case' should be influenced by the aims of the research. However Stake (1995) asserts that the main reason for selecting a case should be to maximise what can be learnt. The need for clear boundaries (geographical, time period, definition, and context) in case selection is also important in order to ensure that the study stays within a reasonable scope (Baxter \& Jack, 2008; Miles \& Huberman, 1994; Stake, 1995).

The focus of this study is factors affecting acutely unwell ward patients. It therefore seemed logical that the ward, where the patient was cared for, and which has clear contextual and geographical boundaries, became the "heart of the study". Stake (1999) warns that it can sometimes be difficult to distinguish between the case and the context. However within this study distinguishing between the case and the context was straightforward. The ward is embedded within the hospital and therefore the context in this study is the hospital.

The specific type of wards selected as cases was informed by the suboptimal care literature reviewed for the concept analysis. There is evidence to suggest that some general ward staff are unfamiliar with the full range of disturbances in physiology affecting the sick patient (NICE, 2007). Furthermore most of the evidence to date regarding suboptimal care has been generated from general surgical and medical wards. It therefore seemed logical to conduct this research within general surgical and general medical ward environments. 


\subsection{Determining the type of case study}

Case study approaches permit the study of single or multiple cases. The selection of the type of case depends upon the purpose of the research enquiry. According to Stake (1995), case studies can be intrinsic, instrumental, or collective. Intrinsic case studies are studied for a unique interest in the case itself and the results have limited transferability. In contrast, the aim of an instrumental case study is to gain insight or understanding of a particular phenomenon. In collective case studies each case is instrumental to learning about the effects of a particular phenomenon (Stake, 1995).

According to Baxter and Jack (2008) collective case studies are similar in nature and description to multiple case studies described by Yin (2003). Yin suggests that in a multiple case study design several cases are examined to understand the similarities and differences between the cases within different contexts. Yin recommends that multiple case study design should follow replication logic (Yin, 2003) whereby individual cases serve as multiple experiments. Each case must be chosen so they "can predict similar results or they can predict contrasting results but for predictable reasons" (Yin, 2003, p. 47). Yin (2003) believes that using multiple case studies adds strength to the final results as he asserts that the analytic conclusions arising from two cases will always be stronger than conclusions drawn from a single case. He also suggests that the contexts of two cases are likely to differ to some degree. If the researcher arrives at the same conclusions from both cases then the ability to generalise findings is strengthened. In contrast, Stake (1994) contends that having more than one case is not "the study of the collective but an instrumental study extended to several cases" (p. 237) in order to lead to a better understanding of the phenomenon under investigation.

A multiple case study approach was chosen for this study because the research question was broad and little is known about factors affecting care of acutely unwell ward patients. Therefore, the more cases studied, the wider the lens. However an additional aim of this study was to make cross site comparisons to determine if context was an overriding influence on factors affecting care of acutely unwell ward patients. In this study determining whether some factors were 
unique or common to either a ward or a hospital was facilitated by using different types of wards in different types of hospitals.

\subsection{Limitations of case study research}

There are two main criticisms levelled at case study research. Firstly, critics of case study research believe that it lacks generalisability (Stake, 1995). Protagonists of case study methodology (Simons, 2009; Stoecker, 1991; Yin, 2003) argue that the purpose of case study research is not to generalise empirically (using statistics or frequencies against a wider population) but to generalise to develop theories, or 'analytic generalisation'. Case studies are therefore a means by which "theoretical explanations of phenomena can be generated" (Sharp, 1998, p. 788). Furthermore, case studies are concerned with the study of the 'particular', to provide a rich description of a setting to inform practice, to establish the value of a case and/or to add knowledge of a specific topic (Bryar, 1999; Simons, 2009; Stake, 1995; Stoecker, 1991).

The second criticism levelled at case study research is the perceived lack of rigour. Proponents of case study research recommend the following strategies be utilised to minimise the problems associated with validity and reliability:

- Use of a protocol to guide data collection (Yin, 2003)

- Use of multiple sources of information (Simons, 2009; Stake, 1995; Yin, 2003)

- Establishing a chain of evidence (Bryar, 1999; Yin, 2003).

With these limitations in mind it was important that this case study design ensured that validity and reliability were attended to. The strategies employed within this research are described in more detail later in this chapter but focused on rigorous data collection techniques, the development of a clear 'audit trail' of the research process, peer consultation regarding data coding, and the development of an analytical framework following the data collection.

\subsection{Developing the research design}

Once the researcher has decided upon the type and number of cases to study the next step is to select research methods. The research methods should also 
match the purpose of the study. Typically in case study, multiple methods for data collection are used. However, careful justification of the methods chosen is required to ensure validity of the study (Jones \& Lyons, 2004).

Given that this was a multiple case study design the development of a study protocol (Appendix 1) ensured a systematic enquiry and a clear process to follow (Gangeness \& Yurkovich, 2006). The use of a protocol is essential in multiple case study designs and increases the reliability of the case study (Yin, 2003). Important inclusions in the protocol are selection of study sites and access arrangements, in addition to data collection procedures.

\subsection{Study sites}

Stake (1995) argues that time for fieldwork is always limited and suggests that cases should be chosen that are "easy to get to and hospitable to our enquiry" ( $p$. 4). The choice of hospital sites in this study was an entirely pragmatic one and was restricted by the limitations of time and funding available. The main condition for selection was that the hospitals needed to differ in size and location. This was in order see if different contexts illuminated different factors effectively.

The two study hospitals were a regional hospital (Hospital 1) and a metropolitan hospital (Hospital 2) in the North Island of New Zealand. The hospitals varied in size, location, management strategies, and support services available for healthcare staff working within the wards. The cases within the context of the hospitals were one general medical ward and one general surgical ward from Hospital 1 (Medical ward A and Surgical ward B) and one general medical ward and one general surgical ward (Medical ward C and Surgical ward D) from Hospital 2. Data was collected over three months from October 2009 until December 2009. Data collection commenced after ethical approval had been granted and when access to the field was negotiated.

\subsection{Ethical approval}

Ethical approval was sought from the New Zealand Health and Disability Multi Region Ethics Committee (Appendix 2). Key ethical principles such as nonmaleficence, beneficence, justice and respect are addressed within the ethics 
approval documentation for contemporary research involving human subjects in New Zealand. This allows the researcher to give appropriate consideration to their ethical stance in the conduct of the proposed study. Particular issues addressed in the ethics application to demonstrate how these key ethical principles were attended to included how the research would ensure the anonymity and confidentiality of participating hospitals and individual participants. Anonymity was assured through ensuring that any identifying features of either the hospital or the individual participants were removed. Confidentiality was assured through restricting access to data to the researcher and supervisors only during the analysis phase of the research. Furthermore, hard copy data were kept in a locked filing cabinet and digital and computer files were password protected. Finally participants were offered reports on the completed research and assured that all significant themes emerging from the data collection and analysis would be included in the final thesis.

\subsection{Gaining access to the field}

As discussed in Chapter 2, suboptimal care of acutely unwell ward patients is associated with shortfalls in care delivery. Both the hospitals and the wards approached to participate in this study had to be prepared for the possibility that perceived shortfalls in care processes might be exposed. The prospect of having shortfalls in care delivery revealed may not have been an attractive prospect to an organisation. Organisations are dynamic and complex places and perceived outsiders may not always be welcome. This is especially relevant if the questions asked are of a sensitive and awkward nature (Okumus, Altinay, \& Roper, 2006). Giving consideration to an 'access plan' for entry to the field and foreshadowing potential issues is advised (Okumus, Altinay, \& Roper, 2007; Woods \& Roberts, 2003).

An initial step within an access plan is to establish contacts with individuals from whom it is necessary to get permission to enter the organisation. Identifying a 'gatekeeper' who can act as a liaison between the researcher and the organisation is seen as very beneficial (Woods \& Roberts, 2003).The initial gatekeeper identified 
at both hospitals was the Director of Nursing (DoN). As the most senior nurse within a hospital each DoN could provide access to the ward areas and ward staff.

'Getting in' (Buchanan, Boddy, \& McCalman, 1988) refers to gaining the trust of the participants you wish to study. Therefore it is essential to be clear about the study purpose, design, and dissemination strategy (Morse \& Field, 1995). Initial contact with the DoNs was made by telephone to arrange a preliminary meeting. Prior to the meeting each DoN was sent a synopsis of the research project via email. Once the meeting was arranged a full research proposal, which detailed the background to the study, the study aim, and objectives, the research outcomes and the dissemination strategy for the research findings, was emailed to each DoN. In the arranged meetings any issues regarding the research proposal were clarified. From this point forward gaining access was unique to each hospital in relation to their locality assessment procedures and also in relation to the requirement for additional information and further discussion.

\subsubsection{Recruitment strategies}

Participants for the study were nursing, medical, and managerial staff at executive, senior and ward level. The DoNs identified potential general medical and surgical wards to approach within each hospital. The ward managers (known as Clinical Nurse Managers [CNMs]) were then approached to determine whether the ward would be interested in participating in the study. In both hospital sites the CNMs of the general surgical and medical wards offered to participate immediately. Meetings were arranged with the CNMs to discuss the study in detail and to determine the best way in which to recruit participants. Developing a relationship with these key stakeholders was vital to the success of the study. As gatekeepers to the wards and the staff that worked on them, their endorsement of the study added credibility and led to recruitment of both nurses and doctors. Specific recruitment strategies included using a poster advertising the study, sitting in at handovers between shifts and explaining the study, sitting in the staffroom at morning and afternoon tea breaks and lunchtimes, attending ward meetings, presenting at medical 'grand round' and lunchtime meetings, medical and surgical 'post take' meetings and House Surgeon (HS) lunchtime meetings. 


\subsubsection{The Participants}

In case study research the sampling strategy is most commonly purposive (Simons, 2009). Purposive sampling entails choosing participants who have a key role in the case. Participants are invited to take part in the study based on their experience and their ability to share their knowledge of that experience in the research context. Participants were identified as registered nurses, doctors, and management staff who came into contact with or had an association with the cases. Also included were the executive management staff (Chief Operating Officer [COO], Director of Nursing [DoN], and Chief Medical Advisor [CMA]) from each hospital. These were included because executive management has the ultimate decisionmaking power within an organisation and therefore has a direct influence on how the wards operate. They were also included to determine if they had a different perspective on which factors affect the care of acutely unwell ward patients.

\subsection{Research methods}

According to Stake (1994) the best way to collect data in a case study is to "place the best brains in the thick of what is going on" (p. 242). This involves spending considerable time on the study site observing and being "personally in contact with activities and operations of the case, reflecting and revising meanings of what is going on"(Stake, 1994, p. 242). In total, 12 weeks were spent in the field with 6 weeks in each hospital. Of the six weeks spent in each hospital, 3 weeks were spent on each ward. During this time detailed field notes were made describing the environment and any observations of how the wards operated. This data was supplemented by informal conversations with staff who worked on the wards. This allowed a detailed account of the wards to be constructed.

\subsubsection{Interviewing and focus groups}

Data which researchers "are unable to see for themselves" (Stake, 1994, p. 242) are collected through documentation review or interviews. Interviews are considered to be the "backbone of case study research" (Gangeness \& Yurkovich, 2006, p. 15) and are conducted to explain, describe, or elaborate on a phenomenon (Yin, 2003). Interviews were chosen to capture the different participants' 
experiences and generate the 'multiple realities' required to fully examine the case (Stake, 1995). In addition to interviews, focus groups were also conducted. The use of focus groups is growing in popularity in nursing research as it "allows the participants in the group to comment, explain, disagree, and share attitudes and experiences" (Curtis \& Redmond, 2006, p. 25). It was also assumed that some participants would feel more comfortable in a one-to-one interview situation and others more comfortable in a focus group. Furthermore ward commitments may preclude removing three or more key personnel from the 'floor' at one time and would be more difficult to facilitate. Allowing participants the choice to participate in either interviews or focus groups may have influenced their decision to participate in the study.

All focus groups were profession specific (nurses with nurses, doctors with doctors, managers with managers). This was in order to ensure participants' emotional safety, as focus groups which contained health staff of different status (particularly the presence of a manager) may have influenced the integrity of responses. A semi-structured approach to interviewing was used in this research. This type of interviewing allows the researcher to outline the main topic areas to be covered but allows flexibility within each interview depending upon the direction each individual interview is taking (Burnard, 2005).

Interview questions need to be clear, unambiguous, and non directive to ensure that participants understand what is being asked of them and to minimise bias (Simons, 2009). Given that factors affecting care of acutely unwell ward patients has not previously been explored, interview questions were deliberately broad, giving participants the opportunity to tell their stories and recount their experiences. In this study participants were asked to relate their experiences of caring for acutely unwell patients within their particular ward environment. The term 'suboptimal care' was deliberately avoided in the interview questions. The researcher had previously observed negative reactions from healthcare staff when this term had been used. Furthermore the aim of this research was not only to determine negative factors affecting acutely unwell ward patients. This research 
also aimed to establish the organisational factors which supported care within a general surgical or medical ward of acutely unwell patients.

There were two main interview questions.

"Can you tell me about caring for an acutely unwell patient which was a positive experience for you? What factors made that experience a positive one?"

And

"Can you tell me about another experience when you cared for an acutely unwell patient that did not go as well. What factors made that experience go less well? What do you think could be done to improve that?"

Participants were asked to discuss what factors had made their experiences positive or negative. As participants told their stories, which recounted incidents, follow up questions probed more deeply into the positive or negative factors highlighted by participants. Where participants required prompting, prompt questions were informed by the antecedents revealed by the concept analysis or by describing scenarios of patient care which were based on the model case constructed for the concept analysis (page 34).

Given that managers at ward and executive level do not give 'hands-on' care to patients, the interview questions were adapted to be more appropriate for these participants. For example "As the (e.g. ward manager, COO) within this organisation what systems and processes do you believe facilitate care of acutely unwell ward patients?"

All nurses, doctors, and managers who agreed to participate in interviews and focus groups were given information sheets outlining the study (Appendices 3 and 4) and were asked to sign consent forms (Appendices 5 and 6) in line with ethical requirements. All interviews and focus groups were recorded using a digital recorder and then transcribed by a hired transcriber who had signed a confidentiality agreement.

Data regarding the number of years nursing participants had been qualified was also collected. This was collected to demonstrate that nursing participants had varying levels of experience. In total eight focus groups and 30 one-to-one interviews were conducted. The collection of demographic data from medical 
participants was not required as their job title denotes their level of experience (e.g. house surgeon is a junior doctor with up to two years experience). The focus groups and interviews lasted from between 20 minutes to 56 minutes with the average time being 35 minutes. The total number of participants from both hospitals was 65 . Table 5 demonstrates the total number of participants by role and organisation in which they worked. Subsequent tables (Table 18, Table 21, Table 24, Table 27, Table 30 and Table 33) groups participants by the specific area in which they worked in each organisation.

Table 5. Breakdown of participants by role and study site

\begin{tabular}{|l|l|l|l|}
\hline \multicolumn{1}{|c|}{ Role } & \multicolumn{1}{|c|}{$\begin{array}{c}\text { Number of } \\
\text { participants }\end{array}$} & \multicolumn{1}{c|}{ Role } & \multicolumn{1}{c|}{$\begin{array}{c}\text { Number of } \\
\text { participants }\end{array}$} \\
\hline Nurses & 19 & Nurses & 14 \\
\hline Doctors & 12 & Doctors & 3 \\
\hline $\begin{array}{l}\text { Ward/Senior } \\
\text { Managers }\end{array}$ & 8 & $\begin{array}{l}\text { Ward/Senior } \\
\text { Managers }\end{array}$ & 4 \\
\hline $\begin{array}{l}\text { Executive } \\
\text { Managers }\end{array}$ & 2 & $\begin{array}{l}\text { Executive } \\
\text { Managers }\end{array}$ & 3 \\
\hline Total & 41 & Total & 24 \\
\hline
\end{tabular}

The mean number of years' experience for nurses working in Hospital 1 was 12.2 years (range 2 years -30 years, SD 8.2). The mean number of years of experience for nurses working in Hospital 2 was 15.1 years (range 3 years - 34 years, SD 10.8).

All participants were given code names associated with their profession, specialty, and hospital they worked at e.g. NursSurgH1 (denoting nurse from surgical ward in Hospital 1). These codes were used on any audiotapes which required transcription, in field notes or when being discussed with thesis supervisors. These code names are used within the thesis if a quotation is taken from a transcript.

\subsubsection{Using documented evidence}

According to Yin (2003) using documentary evidence is a key way of corroborating and augmenting evidence from other sources. Documentary evidence collected and reviewed included hospital policies in relation to patient safety, ward meeting minutes, patient safety documentation (to include new 
service initiatives), staffing rosters, organisational memos to staff and patient acuity measurement tools. The documents were reviewed to determine the authors and source, issues raised, dates of creation, any inconsistencies, and distribution lists. Details of all documentation reviewed are presented in Table 6 and in Chapter 5 . All documentary evidence was reviewed after the analysis of data from interviews and focus groups had occurred.

Table 6. Details of all documents reviewed from both hospitals

\begin{tabular}{|c|c|}
\hline Documents reviewed from Hospital 1 & Documents reviewed from Hospital 2 \\
\hline 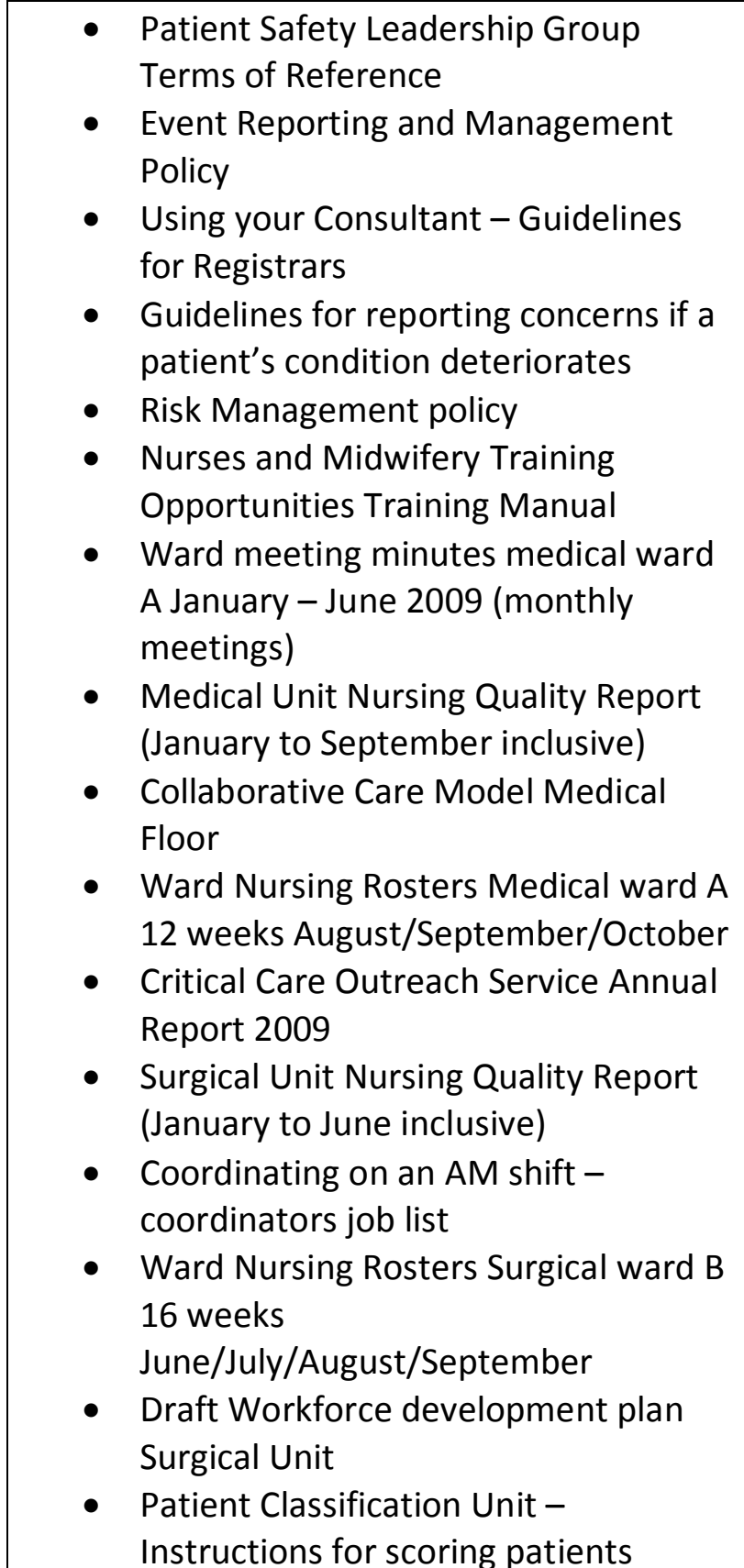 & 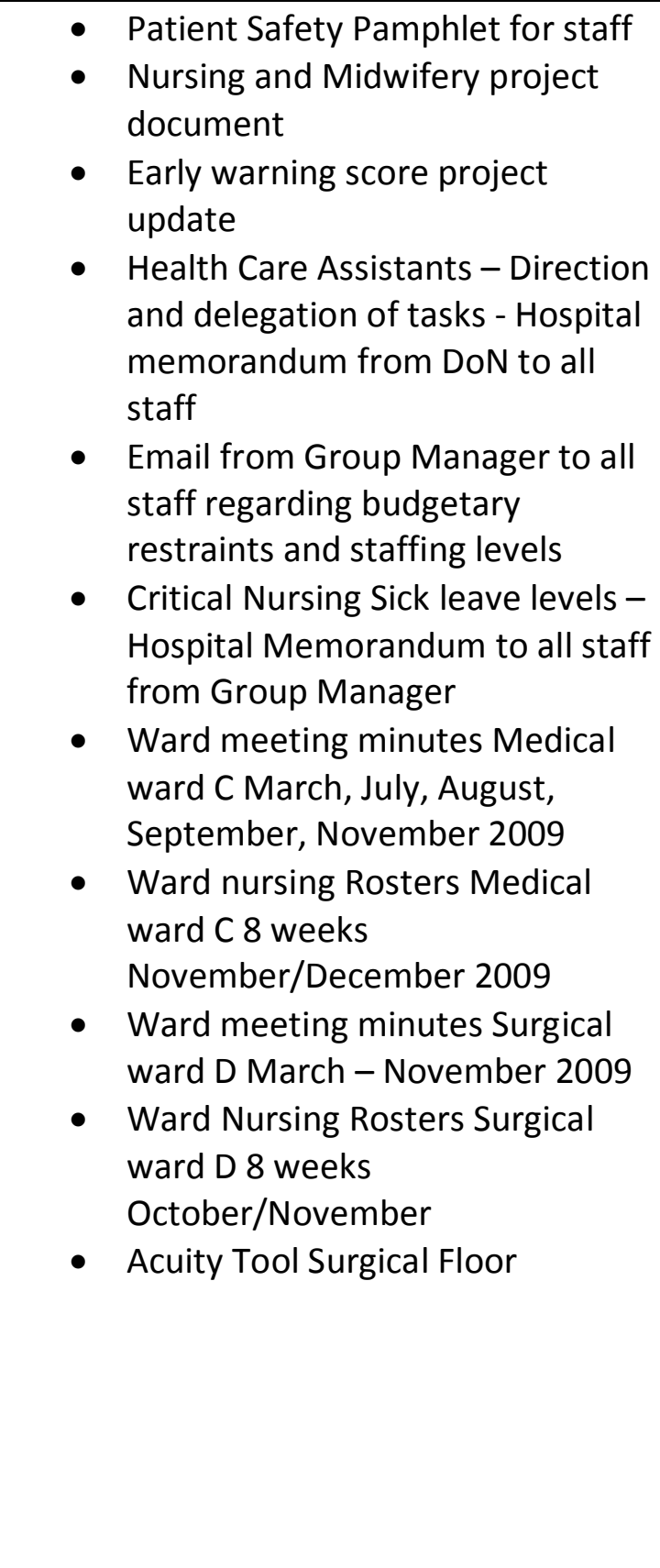 \\
\hline
\end{tabular}




\subsection{Data analysis}

Having a clear plan for analysis was important to the rigour of this study given the multiple sources of data and multiple levels of analysis required. The analysis in this study had five stages and occurred sequentially as follows:

1. All interview and focus group transcripts were coded and an analytical framework was developed.

2. Within-case analysis - individual cases were analysed using the framework derived from the initial coding. Documentation was reviewed to augment data from interviews and focus groups.

3. Within-hospital analysis - individual hospitals were analysed using the analytical framework and documentation used to augment the data as above.

4. Cross-case analysis - findings from the within-case analysis were compared to determine similarities and differences between cases.

5. Cross-hospital analysis - findings from the within-hospital analysis were compared to determine similarities and differences between hospitals.

\subsubsection{Analysis of the individual data sources - deriving the analytical framework}

NVivo 8 computer software was used to facilitate the coding process. Stake notes that "for most important data it will be useful to use pre-established codes but to go through the data separately looking for new ones" (p. 79). In this study four domains were identified from the concept analysis of suboptimal care (patient complexity, healthcare workforce, education, and organisation) to use as the 'preestablished codes'. However patient complexity was not included as a domain for coding in the analysis. This was because the aim of this study was to examine the organisational factors affecting care of acutely unwell ward patients. The degree of complexity patients present with is not a factor which can be changed by the organisation. Therefore the remaining three domains (healthcare workforce, education, and organisation) formed the initial data analysis matrix of codes which 
provided a starting point for the analysis of data. However it was not the intention of this study to test only the factors explicated through the concept analysis. It was also important to see what other factors (if any) emerged. Using a combination of categorical aggregation and pattern matching, the data were reduced (Stake, 1995; Yin, 2003).

Transcripts were repeatedly read and compared to reduce ${ }^{8}$ and collapse data. Data were then clustered under broader categories. Referred to as categorical aggregation, this process involves repeatedly scrutinizing data until categories surface (Stake, 1995). Furthermore Stake contends during analysis the researcher looks for patterns, consistency, and for consistency within certain conditions. Yin (2003) described pattern matching as an effective technique when analysing case study data. If patterns which emerge from the data match predicted patterns, this can strengthen a study's internal validity. Pattern matching occurred between the data from this study and the three remaining domains derived from the concept analysis (healthcare workforce, education, and organisation).

NVivo 8 software uses the term node "to refer to the place where the software stores a category" (Richards, 2009, p.98). In addition NVivo 8 uses a hierarchical system of nodes called tree, parent, and child nodes to represent the relationship between nodes. In this study, as recurrent patterns emerged from the transcripts additional codes were added and organised as child nodes underneath the most appropriate parent nodes. After every four transcripts had been coded, the nodes were reviewed. Hierarchies were further refined at this stage to provide themes (parent nodes) which linked with factors (child nodes) and to reveal how they were related (Charmaz, 2006). In addition some nodes were merged, renamed, or deleted.

As the coding progressed, so the number of nodes decreased, as some were very similar. At every review stage a new coding table was constructed as a Word document to illustrate the changes made to the codes in order to provide an audit

\footnotetext{
${ }^{8}$ The process of selecting, simplifying, focusing, abstracting and transforming the data (Richards, 2009).
} 
trail. In addition, any changes made to the names and descriptions of the codes were noted in NVivo and in a research journal.

Throughout the coding process, thoughts and ideas were recorded as either annotations associated with the individual transcript or as memos linked to a transcript. In addition, a research journal was kept which was added to during the coding process and potential questions were noted to 'ask' the data as the analysis progressed (Richards, 2009).

By the end of the coding process all transcripts had been read and coded using the nodes as reference points until saturation occurred (i.e. no new codes emerged). The next stage was to formulate the analytical framework. This was done by initially examining the theme for similarities which could be combined as one theme and renaming the theme to be more appropriate to the data coded within it. For example, within the domain of healthcare workforce seven themes (communication; teamwork; coping; leadership; roles of doctors and nurses; workload; accountability and responsibility) were reduced to four (workload; teamwork; communication; leadership, accountabilities and roles).The factors were then coded at the most appropriate theme. This process was repeated with each of the domains until the analytical framework was fully developed. Initial findings which were used to derive the analytical framework are presented as Chapter 4.

\subsubsection{Within-case analysis}

Miles and Huberman (1994) describe within-case analysis as the first step in the progression from description to explanation building. Once the analytical framework had been developed each case was analysed individually. Each individual case was reviewed against the analytical framework using only the transcripts of participants who worked on each of the individual cases. The framework was constructed as codes, parent nodes and child nodes within NVivo and all transcripts relevant to each case were re-coded. Once each case had been analysed individually, documentation related to each case was reviewed to augment and corroborate the findings. A description and analysis of each individual case is presented in Chapter 5. 


\subsubsection{Within-hospital analysis}

Once all four individual cases had been coded using the analytical framework, transcripts of participants who were not specifically attached to any of the cases but worked across the organisation (e.g. DoNH2, SenManSPManH1) were coded separately as either Hospital 1 or Hospital 2 using the analytical framework. Like the within-hospital analysis, documentation was reviewed to augment and corroborate the findings from the within-hospital analyses. As with the individual cases, a description and analysis of each case is also presented in Chapter 5.

\subsubsection{Cross-case analysis}

Key themes and factors which emerged from the within-case analysis were mapped against the analytical framework to form a cross-case matrix (see Table 36). Four additional questions guided the analysis at this stage to ensure that the analysis was exhaustive and similarities and differences between cases could emerge:

1. Do the factors that affect the care of acutely unwell ward patients vary within medical and surgical wards within Hospital 1 and Hospital 2?

2. Are there any similarities and differences in factors between Medical ward A and Medical ward C?

3. Are there any similarities and differences in factors between Surgical ward B and Surgical ward D?

4. Are there any similarities and differences in factors between medical and surgical wards across both organisations?

The findings of the cross-case analysis are presented in Chapter 6 .

\subsubsection{Cross-hospital analysis}

Key themes and factors which emerged from the within-hospital analysis were mapped against the analytical framework to form a cross-hospital matrix (see Table 42). The term' key theme' is used to describe themes which were discussed the most by participants. Minor themes reflect those themes least discussed. The 
symbols $(+,-)$ used in the matrix (see Tables 36 to Table 42 ) are a way of demonstrating the orientation of the discussion related to the key theme i.e. discussed in either a positive or negative way. Having a double plus or double negative symbol indicates sufficient discussion by participants to describe the theme as a key theme. A single plus or negative sign denotes a minor theme. This matrix was scrutinised to determine the similarities and differences in factors between Hospital 1 and Hospital 2 from a wider organisational perspective. The findings from this analysis are also presented in Chapter Six.

\subsection{Ensuring methodological rigour}

It is the researcher's responsibility to maintain the rigour of the research they have performed. The concepts of credibility, dependability, and trustworthiness are typically used in qualitative research to describe various aspects of rigour (Graneheim \& Lundman, 2004). In contrast, the terms validity and reliability are used in quantitative research. There are some researchers who suggest that the terms validity and reliability have the same meaning as credibility, dependability and transferability and that there is little to be gained by changing the names of these concepts (Long \& Johnson, 2000; Morse, Barrett, Mayan, Olson, \& Spiers, 2008). Both Yin (2003) and Stake (1995) use the terms validity and reliability associated with maintaining rigorous research processes. Therefore given that both these authors have guided the shape and direction of this multiple case study throughout, the terms validity and reliability will be used to demonstrate how rigour was maintained in this research.

There are three methods suggested by Yin (2003) which help to establish construct validity and reliability of case study evidence. These are using multiple sources of evidence, creating a case study data base and maintaining a chain of evidence.

\subsubsection{Using multiple sources of evidence - methodological triangulation}

As discussed earlier in this chapter, one of the key design aspects of case study research is the use of multiple sources of evidence. Yin (2003) cites the most important advantage of using multiple sources of evidence as the development of 
"converging lines of enquiry" (p. 98). Different sources of evidence (methodological triangulation) are used to corroborate events or facts highlighted by the study. In this study interviews, focus groups and documentation analysis were used to determine the factors that affect the care of acutely unwell ward patients. Using this corroboratory model of data triangulation, any findings or conclusions are likely to be more convincing and accurate (Stake, 1995; Yin, 2003). Furthermore the issue of construct validity is also attended to with the use of multiple sources of evidence (Yin, 2003).

As well as methodological triangulation, investigator triangulation was used to promote validity of the study findings. Investigator triangulation involves using multiple as opposed to singular analysts (Miles \& Huberman, 1994) to "independently analyse the same qualitative data set and then compare their findings" (Patton, 1999, p. 1195). The aim is to reduce the potential bias which may occur when a single investigator carries out all the data collection. Within this case study the consistency of the findings was enhanced by having both thesis supervisors check the coding and a colleague independently code two transcripts. These were then discussed and consensus was reached regarding emerging codes and domains with the aim of ameliorating any "selective perception and blind interpretative bias" (Patton, 1999, p. 1195).

\subsubsection{Creation of a case study database}

The use of multiple sources of evidence leads to the collection of multiple sets of data which must be organised efficiently and effectively. In order to increase reliability of the study Yin (2003) suggests that the case study researcher should produce a formal database in addition to a written report. The creation of the database is to allow other investigators to review the evidence directly without having to go through the entire case report. In this study the qualitative analysis computer software package NVivo 8 was used as the case study database. This software package allows the researcher to import and store documents relevant to the study. Imported and stored documents for this study included all interview and focus group transcriptions, all memos associated with analysis and annotations highlighted within transcripts, all coding categories, excel workbooks listing 
demographic details of participants, numbers, times and places of interviews and focus groups, and an excel worksheet (for each case) listing all documentation analysed for the study (including each document's usual location, authorship, date of issue, nature of the document and distribution list where relevant). The only aspect of the study not stored in NVivo was the handwritten field notes recorded in three notebooks. However these field notes did provide a record of coding, writing and theorising that can be made available for auditing (Tuckett \& Stewart, 2004).

\subsubsection{Maintaining a chain of evidence}

Yin (2003) suggests the process of the study should be so meticulous it can be assured that the evidence presented in the report is the same evidence that was collected in the field. In addition, all evidence should receive appropriate attention and should not be subject to exclusion bias. Several strategies were undertaken to ensure a chain of evidence was maintained within this study.

Firstly, NVivo was used to store all documentation associated with the study and therefore is an accessible repository available for scrutiny. Secondly, the study was conducted as set out in the study protocol. Finally, there is a clear link from the protocol to the collected data, and from the collected data to the analysis, which is then represented in the thesis. Any excerpts from interviews are identified through code names and can therefore be traced back to the transcript stored in NVivo.

\subsubsection{Respondent Validation}

Respondent validation or member checking is suggested as a way of ensuring authenticity of participants' responses and is an example of a strategy that can be applied to indicate a rigorous approach to data collection has been applied (Simons, 2009; Stake, 1995). It also builds in to the case study design some degree of reciprocity with participants (McDonnell, Lloyd Jones, \& Reid, 2000). There are some authors who question the benefit of member checking (Koch \& Harrington, 1998; Sandelowski, 2002). These researchers believe that "members are not always the correct judge" (Sandelowski, 2002, p. 108) of what constitutes valid research and highlight some feasibility issues (Koch \& Harrington, 1998). The feasibility of 
member checking was an issue in this study as there were 40 hours of recorded interviews and focus groups, involving 65 participants. It was not possible within the scope of this study to send back transcripts to this number of participants, some of whom may have left the organisation.

Furthermore it could be argued that the participant in hindsight may not feel the same way when they see what they had said written down, leading them to alter the transcript in some way. It could be argued that interviews or focus groups are "complex social performances" (Allen \& Cloyes, 2005, p. 100) and therefore they should be taken in context, occurring as they do in a particular snapshot of time.

Finally attending to processes which ensured familiarity with the data was important to ensure confidence during the data analysis (Tuckett \& Stewart, 2004). Although the interviews were audio taped and then transcribed by a hired transcriber, each audiotape was listened to on multiple occasions by the researcher and the transcripts corrected where terms were not captured accurately. This involved careful listening, reading, and re-reading of the transcripts.

\subsection{Summary}

Case studies are used when the researcher cannot manipulate the behaviour of those involved in the study, when little is known about the subject and where broad, complex questions have to be addressed in complex circumstances. Using a case study approach allowed a detailed examination of the organisational and ward factors that affect the care of acutely unwell ward patients in the context in which they occur. It also allowed the factors to be examined within individual cases as well as across different cases in order to determine similarities and differences.

Two surgical and two medical wards were the selected cases for this study. These four wards were located in two hospitals in the North Island of New Zealand. Nurses, doctors, and managerial staff from ward to executive level participated in interviews and focus groups. Data from the interviews and focus groups were analysed in five sequential stages. The findings from the five different levels of analysis are presented as Chapters 4, 5 and 6. 


\section{CHAPTER 4: INITIAL FINDINGS: DERIVING THE ANALYTICAL FRAMEWORK}

\subsection{Introduction}

The purpose of this research is to determine the organisational factors affecting care of acutely unwell ward patients in New Zealand (NZ). The concept analysis of suboptimal care presented in Chapter 2 identified four domains that grouped antecedents to suboptimal care of acutely unwell ward patients; these were patient complexity, healthcare workforce, organisation, and education. Accepting that increased patient complexity is not an organisational factor affecting care of acutely unwell ward patients, the three remaining domains guided the initial coding of data collected from interviews and focus groups conducted with nursing, medical, and managerial participants. That initial coding of transcripts from all participants from all four cases and across both organisations led to development of the analytical framework presented in this chapter.

This chapter commences with a summary of the interview questions posed, the data sources used to derive the analytical framework and a summary of the analysis process. The main focus of this chapter however are the findings derived from coding the interview and focus group data. This chapter concludes with presentation of the analytical framework derived from these findings.

\subsection{Analysis of the individual data sources}

This study had five levels of analysis. The analysis of the individual data sources from all participants across both study sites represents the first level of this multi-level analytical strategy. Data used for the analysis of the individual data sources was derived from individual participants' responses to the following questions:

"Can you tell me about caring for an acutely unwell patient which was a positive experience for you? What factors made that experience a positive one?"

And 
"Can you tell me about another experience when you cared for an acutely unwell patient that did not go as well. What factors made that experience go less well? What do you think could be done to improve that?"

As participants discussed their experiences, follow up questions probed more deeply into the positive or negative factors. Where participants required prompting, prompt questions were informed by the antecedents revealed by the concept analysis. These responses were taped, transcribed and then analysed using the techniques of categorical aggregation and pattern matching (Stake, 1995; Yin 2003) facilitated through using NVivo 8 software.

Throughout this chapter quotations taken from transcripts of interviews and focus groups are included. These are used to illustrate the themes and factors. Those participants who could be easily identifiable due to their role within the organization have been aggregated into groups. These groups have then been given a code name related to the professional focus of the group and the hospital that they worked in. Where participant anonymity could be assured each participant is identified by their role, the specialty they worked in (if applicable) and the hospital in which the participant worked. Table 7 lists the code names for participants who are quoted in this chapter.

Table 7. Code names of participants

\section{Participant title}

Code name on transcript

\begin{tabular}{|l|l|}
\hline After Hours Support Nurse 1 Hospital 2 & AHSNurs1H2 \\
\hline After Hours Support Nurse 2 Hospital 2 & AHSNurs2H2 \\
\hline Chief Operating Officer Hospital 1 & EMTH1 \\
\hline Chief Operating Officer Hospital 2 & EMTH2 \\
\hline Clinical Nurse Educator Medicine Hospital 1 & CNEMedH1 \\
\hline Clinical Nurse Educator Surgery Hospital 2 & CNESurgH2 \\
\hline Clinical Nurse Manager Medicine Hospital 2 & CNMMedH2 \\
\hline Clinical Nurse Manager Surgery Hospital 1 & CNMSurgH1 \\
\hline Clinical Nurse Manager Surgery Hospital 2 & CNMSurgH2 \\
\hline Chief Medical Advisor Hospital 2 & EMTH2 \\
\hline Director of Nursing Hospital 1 & EMTH1 \\
\hline Director of Nursing Hospital 2 & EMTH2 \\
\hline Focus Group House Surgeons Hospital 1 & FGHSX9H1 \\
\hline Focus Group Nurses Medicine x4 Hospital 2 & FGNursesMedx4H2 \\
\hline Focus Group Senior Nurses x2 Hospital 1 & FGSNx2H1 \\
\hline Focus Group Nurses Surgical x4 Hospital 1 & FGNursesSurgx4H1 \\
\hline
\end{tabular}


Table 7 (continued). Code names of participants

\begin{tabular}{|l|l|}
\hline Participant title & Code name on transcript \\
\hline Focus Group Nurses Surgical x2 Hospital 2 & FGNursesSurgx2H2 \\
\hline House Surgeon Medicine Hospital 2 & HSMedH2 \\
\hline House Surgeon Surgery Hospital 2 & HSSurgH2 \\
\hline Medical Registrar Hospital 2 & MedRegH2 \\
\hline Medical Registrar Hospital 1 & MedRegH1 \\
\hline Nurse B Medicine Hospital 1 & NursBMedH1 \\
\hline Nurse Surgical 1 Hospital 1 & NursSurg1H2 \\
\hline Senior Doctor Hospital 1 & SDH1 \\
\hline Senior Manager Medicine Hospital 1 & SenManMedH1 \\
\hline Senior Manager Medicine Hospital 2 & SenManMedH2 \\
\hline Senior Manager Specialist Service Hospital 1 & SenManSPH1 \\
\hline Senior Manager Surgery Hospital 2 & SenManSurgH2 \\
\hline Senior Registrar Medicine Hospital 1 & SenRegMedH1 \\
\hline Senior Registrar Surgical Hospital 1 & SenRegSurgH1 \\
\hline
\end{tabular}

When questions were posed, the researcher's initials appear after the text. Where participants have used names or acronyms these have been removed and [NAME/ACRONYM REMOVED] has been substituted.

\subsection{Healthcare workforce}

The main domain to emerge from the data was healthcare workforce. Within this domain four themes emerged: workload; teamwork; communication; and leadership, accountability and roles.

\subsubsection{Workload}

The major theme to emerge from this research was the issue of workload. Factors affecting workload were: having time and coping; task focus; allocation; nurse to patient ratio; and skill mix. Workload was affected by the complexity of the patient population and the number of nurses or medical staff on duty, which impacted on the amount of time available to care for patients.

"Well if you've got five patients and they've all got you know multiple co morbidities you know and they're needing a lot of things done to them so you really can't concentrate on one patient because you've got another four" (FGNursesMedx4H2)

And

"I was sitting on one ward on the phone to the other ward and there's like probably about six nurses lining up behind me with like charts and things I need to do" (HSSurgH2). 
This heavy workload was believed to impact on nurses' willingness and ability to increase observations or spend additional time with patients who were acutely unwell.

"They think their workload is very, very high and everything comes down to their workload I think. If you ask them to do something that's new or takes more time, especially if you ask them to increase their observations 99\% of the time they'll always throw back at you but my acuity is, have you seen my workload" (CNEMedH1)

Furthermore, supervising student nurses or other members of staff impacted on nurses' individual workloads, limiting the ability to spend adequate time with patients.

"So it's hard work having a student and if you've just got a couple of well not complicated but patients that just need that little bit more oomph of you know busyness where you don't muck around you get in and do what you have to do, because in the back of the mind we have always got to teach them and explain what I'm doing to them" (FGNursesSurgx6H1)

Because workload was perceived to be onerous some nursing staff resorted to a task focused approach, seeing their workload as a list of jobs that needed to be completed before the end of a shift. This was believed to be a barrier to caring for acutely unwell ward patients as nurses were more concerned about working through the list rather than looking for possible signs of patient deterioration.

"And often they're so sort of focused on trying to get their jobs done and their tasks done that they tend to sort of think more about tasks and they're more focused on getting all that done and that can definitely affect care because you know I've come along and I'll be worried about a patient and I'll go and investigate that patient and I'll pick up things that haven't been caught up with" (CNMMedH2)

A heavy workload was sometimes as a result of poor patient allocation practices. Some nursing staff were given overly heavy patient loads with limited ability for reallocation should their patient acuity levels change, for example if a patient were to become acutely unwell. Some management participants acknowledged these allocation practices as a factor which impacted on the care of 
acutely unwell ward patients and reported that steps had been taken to redress this issue.

"I've encouraged the nurses when they come on and they've assessed their patient after handover, if they're not happy with the allocation to talk to the coordinator who's been allocated and reallocate the load. Sometimes it is done at the end of the shift, sometimes that's done after handover, and I think nurses are getting better at saying hang on and to question because I think as I say there's a lot of things that just are done here and it's not questioned why" (SenManSPH1)

Coping with a heavy workload was also a factor discussed by participants at all levels. The ability of individual nurses to cope with workload challenges was viewed as a measure of professional competence and therefore some staff were reluctant to ask for help. For medical participants the inability to adequately manage their workload was viewed as possibly being detrimental to their career progression, therefore some were unwilling to ask for help.

"The house surgeons then don't feel comfortable all the time to call their registrar because it's seen as they're not coping" (EMTH2)

Junior doctors 'coping' and perceived reluctance to ask for help was viewed by the nursing participants as a factor which affected the care of acutely unwell ward patients. Junior nurses not asking for help was a source of frustration for more senior nurses when nurses continued to 'cope', didn't ask for help, and patients were potentially put at risk.

"Because nurses are exceptionally good at shooting themselves in the foot. Because they will do what they have to do to get it done you know" (SenManSurgH2)

Coping was also related to staffing levels.

"Oh well they'll manage, oh well they can manage can't they is the general response" (SenManMedH2)

When nursing participants felt that they could not cope with the ward workload and asked for additional resources, the response was sometimes unsupportive and they were expected to cope with the resources that they had. Nursing participants stated that shifts were often inadequately staffed. Getting replacement staff to increase the staffing numbers occurred infrequently. Some nursing participants 
believed inadequate staffing levels could lead to the possibility that acutely unwell patients may not be detected.

"We often have shifts there where they don't get covered and they are given a heavy load and they are busy. And that would happen more frequently than not actually. You're probably looking at, I would say $20 \%$ of the time, $20-30 \%$ of the time you would be staffing on sub [optimal] staffing numbers. So they're looking at picking up six patients which you would normally give them, they would normally get between four and five. So they, 30\% of the time they'd be looking at picking up a heavy load" (CNMMedH2)

Although unhappy that staffing shortfalls occurred, nursing participants were very pragmatic in their attitude towards finding replacement staff. Many expressed their understanding that nurses as a resource was very limited and that there may not be any replacements.

"I think they're short all round as well. This is the problem it's short all round. You know trying to get blood out of a stone" (FGNursesMedx4H2)

The view that there were simply not enough nursing staff to care for patients was very apparent when participants were asked what resource, system or process would help them care for acutely unwell ward patients. The resounding response from the nursing participants was more staff. Most wanted more nursing staff, however others requested more Health Care Assistants (HCAs) or more medical staff.

"I'd ask Santa for a doctor on the ward all the while, a consultant that could be easily accessible 24 hours a day and a one nurse to four [patient] ratio. One nurse on the floor and adequate seniors to juniors and a coordinator" (AHSNursH2)

Nursing participants expressed the view that with adequate resources acutely unwell ward patients could be managed appropriately. In contrast, some executive management participants stated that nurses' perceptions of their workload were not entirely realistic. One member of the EMT expressed the viewpoint that nurses had talked themselves in to a 'tyranny of busyness' which impacted on their ability to manage their workloads.

"We've got more nurses today than what we've ever had at any time in our history, we've also got more sick patients and the turnaround, 
the churn is far greater. I think we need to stop using busyness or almost relying on the tyranny of busyness as an excuse. This is our job" (EMTH2)

Furthermore the issue of skill mix was also viewed from different perspectives, depending upon the participants' role in the organisation.

"We've got a very high number of senior nurses in this organisation. Our ratio of senior to junior nurses is high, I think when [NAME REMOVED] last looked at it I think $80 \%$ of our nurses are level five or above on the pay scale. So that would indicate to you that our registered nurse workforce is an experienced nurse workforce" (EMTH2)

Nursing participants viewed skill mix as poor and detrimental to adequate care of acutely unwell ward patients, whereas executive management participants believed skill mix to be appropriate. Table 8 summarises the factors to emerge from the theme of workload.

Table 8. Factors to emerge from the theme of workload

\begin{tabular}{|l|l|}
\hline Theme & Factors \\
\hline Workload & Having time and coping \\
& Task focus \\
& Allocation \\
& Nurse to patient ratio \\
& Skill mix \\
\hline
\end{tabular}

\subsubsection{Teamwork}

Within the theme of teamwork five factors were identified: support; relationships; credibility and trust; clinical experience; and being confident. Nursing, medical and managerial participants believed that providing support within teams was essential in relation to caring for acutely unwell ward patients. When junior nursing participants felt 'out of their depth', the ability to call on more senior nurses to assist them in caring for these patients was seen as very important. When this support was not forthcoming, junior participants felt isolated and scared. Support was not always in the form of assisting with direct patient care. Support could also be removing some of the workload to give the nurse the necessary space to care for the patient. It also came in the form of teaching and debriefing regarding stressful situations. 
Within teams, relationships could become strained at busy times, and when other nurses such as the critical care outreach service helped ward nurses care for acutely unwell ward patients.

"we do have a culture of nurses that some of them if you ask them to do something or you ask them why they haven't done it they see it as a personal affront, you're having a go at them" (CNEMedH1)

Some ward nurses felt that the critical care outreach nurses undermined their own care of the patients and that this service took over. This led to defensive behaviour on both sides which was not conducive to good teamwork or to providing effective nursing care to acutely unwell ward patients. Both nursing and medical participants described the presence of distinct professional hierarchies between doctors as affecting relationships, with fear of being admonished affecting the ability of junior medical staff to refer patients to more senior members of the team.

"Doctors are very reluctant to ask for help to go up the line. House surgeons are out of their depth and are scared to ask a registrar and the registrars are scared to ask the consultant" (AHSNurs1H2)

Participants believed this was prevalent within doctors' own specialty and across different specialties. Both nursing and medical participants felt that teamwork between professions and specialties was often poor, which directly affected how acutely unwell ward patients were cared for. Fear of contacting specific specialties due to the personalities of personnel who worked in these areas led to poor working relationships and was detrimental to effective team working. Given that acutely unwell ward patients are most likely to require the specialty skills of intensivists, it was not surprising that the main area highlighted in relation to this issue in both hospitals was the intensive care unit (ICU).

"But because there's only so many beds and there are different personalities in the department access can be problematic. And I think because there's different personalities in the department some people are afraid to contact ICU (SenRegMedH1)

The exception to this was in emergency situations such as cardiac arrests, when team working was perceived by participants as being effective.

Problems in relationships between nurses and doctors were frequently highlighted by nursing participants as directly affecting their ability to work as a 
team caring for acutely unwell ward patients. Medical staff having a collegial attitude towards nursing staff, demonstrated through asking their opinion and seeking their advice, was viewed as an extremely important factor in building good working relationships.

"Some of it comes from, it sometimes depends on what the registrars are like. If the registrars sort of come to the nursing staff a lot and ask their opinion and things like that, and the consultant does that it makes a huge difference to the actual team" (CNMSurgH2)

The nursing participants believed it was important that the medical staff both acknowledge and value their nursing skills and expertise. When this occurred, nursing participants consulted medical staff more freely and trusted their decision making.

The length of a rotation and time spent on a particular ward was important for the nurses to become familiar with the house surgeons. Nursing participants felt it was also important to allow the house surgeons to get to know and appreciate the level of the nursing staff's experience. When nursing participants felt individuals did not value their views or opinions they avoided these individuals.

"after dealing with that house surgeon that night I didn't really deal with him a lot any more directly after that even when they were on the day shift I just normally went to one of the other colleagues or the med reg" (NursBMedH1)

Avoiding interactions with house surgeons specifically was problematic given that house surgeons are usually the first doctor that should be contacted in regard to problems related to the care of acutely unwell ward patients.

Both nursing and medical participants discussed the issue of clinical credibility and trust. Whether the nurse or the doctor knew 'what they were talking about' in relation to unwell patients affected the response they received when communicating problems and, therefore, affected team working. Nursing participants perceived that medical staff viewed senior nurses and critical care outreach nurses as more credible than ward nursing staff. This was because these nurses were seen to get a more immediate response from medical staff than ward nurses did, which frustrated both the ward nurses and the senior and critical care outreach nurses. 
"they'll say you ring him. But you've rung him. But he ignores me but as soon you say CCON's ringing he'll come. And he does and it's annoying and it infuriates me because I heard a nurse speak to a registrar. I said to her you ring him. And they spoke to the registrar and the registrar's ignored them and put the phone down"(CNEMedH1)

Being confident was associated with the level of clinical experience or clinical exposure of either the nurse or junior doctor. When nurses or doctors lacked confidence they were seen to avoid confronting a difficult situation. Lacking confidence also impacted on both nursing and medical staffs' ability to ask for help.

"Not having the confidence or the depth of knowledge that they need to make a decision so for them it's confidence. So they may have a patient that is unwell but then don't have the confidence to do anything more about that not to ask another nursing colleague or how to think about where to from here "(SenManSPH1)

And

"Junior doctors, the skill level coming out is less experienced in acute management and hasn't had as much exposure in medical school to hands on sort of day-to-day stuff in terms of like acute patient management. So people feel less confident or have never actually seen things" (SDH1)

Participants believed that this affected acutely unwell ward patients as individuals struggled on alone and could potentially make poor decisions regarding patient management. Table 9 summarises the factors to emerge from the theme of teamwork.

Table 9. Factors to emerge from the theme of teamwork

\begin{tabular}{|l|l|}
\hline Theme & Factors \\
\hline Teamwork & Relationships \\
& Support \\
& Credibility and Trust \\
& Clinical experience \\
& Being confident \\
\hline
\end{tabular}

\subsubsection{Communication}

A key theme that emerged from the data from participants in all roles was communication. The two main areas of concern were breakdown in 
communication and responding to requests for review. Both nursing and medical participants raised concerns regarding communication but from differing perspectives. There was considerable tension apparent between both professional groups when it came to communication regarding the care of acutely unwell ward patients. The participant doctors stated that they were often not told in a timely fashion that patients were deteriorating or were not told at all. The delayed or nonexistent referral by the nursing staff and subsequent delayed review by the doctors adversely affected the care acutely unwell patients received.

"I realised I had a septic patient that wasn't peeing sort of about six hours later than I could have realised, if I'd been told about it three hours earlier I would have been able to change the drip three hours earlier"(FGHSx9H1)

When nurses did refer in a timely fashion the amount of information that the doctors received regarding the patient was variable. This limited the doctors' ability to triage the patients into those who required urgent referral in comparison to those who could wait. Medical participants stated that their expectations regarding the level of detail required for an adequate handover were not unrealistic and was no more than was expected of them by their senior colleagues.

"And we're not asking anything that we wouldn't do ourselves. Like that's what we would expect as a baseline if we were ringing a registrar. Generally you wouldn't have the notes in front of you [only] if you knew the patient really well, because it's a gamble and so you have the obs chart and the drugs chart available. Otherwise they're going to keep asking questions you can't answer and you look like a dick" (FGHSx9H1)

This lack of information was often compounded by nursing staff not being available to accompany the doctors when they came to urgently review the patient as requested. Medical participants discussed the frustration of coming to review the patient and not being able to locate the appropriate information such as the patient's notes and observation charts.

"She was sitting having a cup of tea in the tearoom and the notes were nowhere to be found and the drug charts were nowhere to be found, or anything. And I was just, I was a bit agro and I was like you know if you expect me to come here urgently you need to be prepared to discuss the patient and have the stuff available" (FGHSx9H1) 
The doctors then had to spend their limited time looking for the nurse looking after the patient or the relevant documentation to help them adequately assess the patient and formulate a treatment plan.

Nursing participants' concerns regarding communication focused more on who to call and getting a response. Nursing participants talked about the difficulty of knowing exactly the appropriate doctor to call given doctors' protected teaching time, the complex way that medical teams were organised and how on-call rosters are organised.

"I mean sometimes they'll say it's no it's not me and the other girl will say it's not me. And it's like could you guys talk to each other" (FGNursesSurgx4H1)

This was believed to be a factor adversely affecting the care of acutely unwell ward patients, as nursing participants felt they wasted valuable time trying to call the correct doctor. In contrast to the medical participants, nursing participants felt that even when they used communication tools such as SBAR, which were designed to ensure that appropriate and sufficiently detailed information is handed over, doctors failed to respond to their requests for review of acutely unwell patients.

"We do have situations here with some of the house surgeons that if you ring them and we've got the SBAR that that we go down as to what the situation is and everything else, they will just not listen to you" (NursSurg1H2)

Conversely some nursing participants fully understood that in order to get a quick and adequate response from medical staff the handover of information had to be accurate and detailed.

"But that's why you need to be able to articulate what you think is wrong with the patient for the other person to take it seriously or to understand the urgency" (FGNursesSurgx2H2)

However both nursing and medical participants felt that nurses were guilty of 'over paging', and building urgency into requests which was not justified. Consequently, many doctors were unsure of which requests for urgent review were genuine and some had become 'blunted' to requests to see patients.

"The house surgeons don't answer their page because they say nurses page me constantly for silly old things and just know that it's not important so why should I bother answering" (EMTH2) 
When nursing participants could not get a response from the house surgeons, hospital policy in both organisations dictated that they should escalate care to the next level of doctor i.e. the registrar, or even the consultant. However this too was an issue, as nursing participants perceived that junior nurses lacked confidence in approaching senior doctors.

I think some nurses will go so far but you know they'll try and get hold of the registrar but if they can't get hold of the registrar they don't always have the assertion to ring the consultant and don't feel comfortable about doing that" CNESurgH2

Furthermore nursing participants described scenarios when even experienced nurses had encountered hostility when trying to escalate care. Table 10 summarises the factors to emerge from the theme of communication.

Table 10. Factors to emerge from the theme of communication

\section{Theme}

Communication

\section{Factors}

Responding

Breakdown in communication

\subsubsection{Leadership, accountability and roles}

Within this theme four factors were identified: leadership roles; seeing the bigger picture; being accountable; and the roles of doctors and nurses. Leadership roles focussed mainly on nursing leadership roles and encompassed shift coordination, ward leadership, and role clarity. Shift coordination was perceived by many participants as one of the key elements which affected the care of acutely unwell ward patients. During the morning shift the wards were coordinated by the ward manager, whose primary responsibility was to run the ward and not necessarily to participate in direct patient care. However on the afternoon and night shifts the most senior member of staff coordinated the shift. This was seen as extremely problematic by nursing and ward and senior management participants as these senior nurses were also expected to take a patient load. Theoretically the coordinator's patient load was supposed to be 'lighter', but in reality the coordinator invariably took some of the sicker patients as well as providing support for junior members of staff. This was in addition to the responsibilities of coordination, such as liaising with the bed manager, organising staff for subsequent 
shifts, and generally having an overview of the ward. According to participants the coordinator's ability to have 'peripheral vision' was severely impaired.

"The coordinator's too busy with their own workload, they're not aware of deteriorations happening in other rooms with other staff. That's another reason for the management not being optimal" (FGNursesSurgx2H2)

Nursing participants also noted that the quality of coordination was variable. Some participants believed that was due to a lack of clear expectations of and training for the role.

"...how do we build up the skills in these nurses so when they do take, start taking charge of shifts they know what they're doing and what the responsibilities are and we've given them some tools to manage that" (FGSNx2H1)

At the time that this research was conducted neither organisation provided any specific training for shift coordination although all four wards had 'job lists' for the coordinator. Nursing participants stated that coordinating was seen as extremely hard work and very stressful. As such it was an unpopular role, with nurses refusing to coordinate at times, which required specific action from one ward manager.

"People were refusing and well arguing about who was going to coordinate so we just starting allocating it, divvying it out" (CNMSurgH1)

Ward leadership was seen as a factor which may affect the care of acutely unwell ward patients. This was in relation to clarity of expectations within the role, particularly regarding role accountabilities and responsibilities. Senior management participants saw some ward leadership as ineffective, particularly in relation to ensuring safe and effective care.

"But it's the accountability I'm talking about is actually not putting your money where your mouth is but it's like it's actually okay checking on what people say they're doing. It's a little bit of, yeah it's like okay so you're saying that you're delivering really good care but how do I know you're doing that?" (EMTH1)

However this shortfall had been recognised and both organisations had recently reviewed ward management roles and responsibilities to ensure that accountabilities were adequately reflected. 
Being accountable for patient care and seeing the bigger picture associated with patient care were also important factors. Nursing, medical and managerial participants stated that some nursing and medical staff were very quick to hand over accountability of their patients to someone else. This handing over of accountability was perceived as an issue between nurses and doctors, and junior doctors and senior doctors.

"And I sometimes get that impression from the house surgeons that it's almost like rather than me thinking about it is just quicker to house surgeon page and that's all and it's no longer your problem. It's not your responsibility" (SDH1)

Both nursing and medical participants raised concerns regarding clinicians not seeing 'the bigger picture' and simply focusing on the care to be delivered during their shift. These participants saw this as potentially detrimental to the care of acutely unwell patients. No one appeared to take an overview of the patient which could mean that some important patient problems could be missed.

"So I find that you know there are some great house surgeons but I think and I'm not saying the rest of them are bad, I just think that the ownership is not there. The ownership of your patient is not there. They don't have that. Their shift ends, it's someone else's problem" (SenRegSurgH1)

And

"And that's again where you get unstuck is that whole thing you know, people are just managing for that period and they don't go back and where they've been, what's been done, who followed up on what" (FGSNx2H1)

Furthermore, some nursing participants were seen as simply 'going through the motions' of care and not thinking complex issues through sufficiently, for example in relation to taking and recording observations.

"They're not reacting to the observations they don't feel it's their job to react to the observation" (CNEMedH1)

Medical and nursing staff not reacting to the observations or dealing with abnormal findings was seen to be factor in why there were significant delays in getting the appropriate management plans for acutely unwell ward patients.

Both nursing and medical participants talked about the blurring of roles between doctors and nurses as nurses took on additional roles which had 
previously been seen as the role of the doctor, e.g. ordering and performing blood tests. However the extent to which nurses were willing to take on these additional roles varied between specialties and hospitals. This was viewed as a factor which influenced the care of acutely unwell patients as there was an expectation from some medical staff that these additional responsibilities had been universally accepted. When nurses did not perform these additional tasks there was confusion as to who was then responsible.

Conversely some nursing and medical participants stated that nurses had traditional views of the doctor role and deferred all decision making to the doctor, believing them to know best regarding patient care. In addition some nursing participants felt that the nursing role which traditionally involved important components such as attending to personal hygiene such as showering a patient was being replaced by more technical aspects of care. However some senior managers felt that some nursing staff focused too much on this traditional aspect of the role to the detriment of managing the more complex aspects of a patient's care. Table 11 summarises the factors to emerge from the theme of leadership, accountability and roles.

Table 11. Factors to emerge from the theme of leadership, accountability and roles

\begin{tabular}{|l|l|}
\hline Theme & Factors \\
\hline Leadership, accountability and roles & Leadership roles \\
& Being accountable \\
& Seeing the bigger picture \\
& Roles of doctors and nurses \\
\hline
\end{tabular}

\subsection{Organisation}

The second domain elicited from the interview and focus group data was the domain of organisation. Themes within this domain were: organisation of $24 / 7$ care; organisational systems and processes; and culture and environment.

\subsubsection{Organisation of $24 / 7$ care}

Participants from all roles and from both hospitals discussed the reduced number of staff and dearth of senior support after hours. Participants described an environment where there was a lack of both senior medical and nursing support, 
which they believed was a factor which affected the care of acutely unwell ward patients. Charge nurse managers (CNM) worked from $0730-1700$, Monday to Friday, with no 'appointed' ward management staff on in the afternoons and overnight. The most senior doctor available on site after hours was the registrar, which compounded the sense of lack of support, especially since the registrar would often be busy seeing patients in the emergency department (ED) or operating in theatre. The lack of ancillary staff after hours meant that the nursing staff took on additional roles such as answering the telephone, which impacted on the time available to deliver care to patients.

Participants from all professions also discussed the reduced number of staff rostered on after hours. The ratio of doctors to patients overnight was described as very low. When 'on call', doctors described covering a significant number of patients throughout the whole hospital, the majority of whom they had no previous knowledge of. This meant they had to prioritise who was the most in need of review. This relied heavily on the information given to them by nursing and more junior medical staff.

Furthermore the lack of clear treatment plans determined by the patient's admitting team led to confusion in terms of patient management. Some patients had significant time periods between reviews, as junior doctors were responsible for so many patients when on call.

"Prioritising is the key word when you are on call. Who do you need to see urgently, who is going to die and who can I see. Can I see, do I really need to see the 20-year-old lady who's come into hospital five times with abdominal pain or do I need to see the 85-year-old lady who suddenly started having abdominal pain and vomiting? The answer is only God knows you know and you can make your best judgment and get caught out of sort of" (HSMedH2)

And

"On the weekend patients have a longer period of time when they're not seen and so that's the period of time when they more often become unwell and the house surgeons are called to see them and make decisions about what's wrong" (MedReg1H2)

Nursing participants described afternoon and night shifts as 'horrendous' or a 'nightmare'. This was because the overall nursing workload remained the same yet 
there were fewer nurses rostered to care for the patients. This was felt to affect patient safety.

"It makes it very hard when they might have you know on a night shift they may have 12, 13, 14 patients it makes it very difficult for them to get in to see their patients as often as they would or check that things are okay" (CNMMedH1)

Continuity of care was also an issue which participants thought affected the care of acutely unwell patients. Shift work was thought to affect continuity of care by disrupting the flow of information between members of the healthcare team.

"Nowadays because of many, many reasons one of them might be the RDA contract, that there isn't the same continuity of care. And you may be looking after patients that you don't, that you've never actually met. And if you're lucky you've had a formal handover" (EMTH2)

The lack of support after hours was acknowledged by senior management participants as an issue. Senior management participants described after hours as a vulnerable time for patients but discussed support services such as critical care outreach as a means of providing additional support for staff.

"CCON was originally brought in to acknowledge the fact that after hours is the time of greatest liability with patient care because you don't have the senior medical or nursing support around" (SenManMedH1)

However, whilst nursing participants acknowledged this support in caring for acutely unwell ward patients, participants felt that the outreach nurses were often busy themselves and were often unable to provide the support they needed. Table 12 summarises the key factors to emerge from the theme of organisation of $24 / 7$ care

Table 12. Factors to emerge from the theme of organisation of $24 / 7$ care

\begin{tabular}{|l|l|}
\hline Theme & Factors \\
\hline Organisation of 24/7 services & $\begin{array}{l}\text { Support } \\
\text { Continuity of care }\end{array}$ \\
\hline
\end{tabular}

\subsubsection{Organisational systems and processes of care}

Within the theme of organisational systems and processes of care, participants identified service initiatives, organisational policies, and access as 
important factors. Service initiatives were new services or resources introduced by the organisation with the intention of supporting staff in their care of acutely unwell ward patients. Service initiatives identified by participants were critical care outreach services (CCOS), early warning scores (EWS), and high dependency units (HDU).

There were mixed views as to the value of the critical care outreach service. Some nursing participants valued their support and advice when caring for deteriorating patients. The CCOS were viewed as being able to get action from medical staff in a much more timely fashion and were perceived to be able to bypass the traditional referral processes. However some staff felt that CCOS took over their patient care instead of supporting them. Nursing participants felt this then undermined their nursing care abilities. As far as the senior and executive management were concerned, the role of CCOS was to manage clinical risk.

"I guess organisationally and this is what, what I thought about when I became involved in it was (a) the slippage, (b) was the fact that yeah we're getting people coming in who needed some extra stuff and (c) was the fact that we couldn't mitigate the risk by any other way really" (EMTH1)

Senior and executive management participants viewed CCOS as a team of highly educated and experienced nurses who had the responsibility of supporting both nurses and doctors in their care of acutely unwell ward patients. In conjunction with the CCOS, participants discussed the use of EWS.

Participants in all roles also expressed mixed views regarding the use of EWS. In one hospital, management participants described the use of the early warning scores as a part of the quality programme, with regular audits being carried out to check compliance. For some nursing participants EWS was an adjunct to clinical assessment of a patient, but was also a way of getting attention from medical staff where previously they may have been ignored. It provided additional data to assist nurses in articulating that their patient was unwell. However some medical and critical care outreach participants believed that EWS prevented staff from engaging with the observations they were taking.

"And like I've had someone call me and said just letting you know this person's early warning score is whatever it was and then I was 
okay and they're about to hang up and I'm like hold on, hold on. So why are they in hospital, why is their early warning score that and yep have to ask all the questions" (FGHSX9H1)

These medical and CCOS participants believed that EWS should not be implemented without sufficient training and support to ensure that nursing staff fully understood the supporting rationale for using the score.

The final service initiative to be discussed by staff was high dependency care. Nursing participants believed that having an accessible HDU with an increased nurse to patient ratio where acutely unwell patients could be co-located would mean that these patients could be more appropriately cared for. Nursing staff believed that the ward was an unsuitable environment to care for patients when they became acutely unwell and that HDU was the best environment. Some participants expressed frustration when patients were not moved as they believed there was not sufficient time for them to adequately care for acutely unwell patients and look after the rest of their patient load.

Organisational policies and processes which participants believed affected the care of acutely unwell ward patients were: the management of outliers; the model of care used in ward areas; acuity measures; practice restrictions; and documentation. Nursing and medical participants described their concerns regarding the difficulties in managing outliers. Nursing participants believed that the organisation of care was different between surgical and medical wards. Surgical nursing participants described surgeons doing a brief ward round in the morning, writing a treatment plan and then revisiting in the evening after they had finished operating. When medical outliers were placed on surgical wards, they experienced frustration at getting hold of medical doctors, the length, and format of ward rounds, and also the way physicians managed their patients.

"They'll do a morning round and they'll decide on something. They'll do an afternoon round and they decide on something. And yeah it just seems to go on and on forever" (FGNursesSurgx4H1)

Both nursing and medical participants believed being an outlier directly affected how the patient was cared for because of the extra time required to physically go and see outliers. One medical participant stated that up to $30 \%$ of medical patients 
within his organisation were outliers, which had negative ramifications for both the patients and the staff caring for them. Outliers could be scattered throughout the hospital on wards where the nursing staff did not have the knowledge or expertise to care for them.

"But it does mean that management of patients is adversely affected because we've got patients on the medical ward, we have patients on the surgical ward, on the neurosurgery ward, on the respiratory, on the renal ward, on the gynaecology ward. We get patients down there. And then the nurses on those wards tend to be sub specialised nurses and they're not as experienced at dealing with some of the problems which turn up in their ward under general medicine" (MedReg1H1)

Because patients were so widely spread junior doctors had to triage which were the most important tasks to attend to or try to 'package' care, i.e. do all the jobs at the same time to prevent having to spend time revisiting, as this was time consuming and impacted on an already busy workload.

The current model of care, namely individual patient allocation, was also suggested by the management participants as being a factor which affected the care of acutely unwell ward patients. Both hospitals were in the process of introducing team nursing as they felt that this might improve patient care, since team nursing promoted senior staff supporting junior staff and prevented staff members working in isolation.

Nursing participants described the acuity measurement tools in their organisations as a means of demonstrating how sick their ward patient population was. The intention of this data was to calculate the number of staff required to manage the level of patient acuity within the ward. Some nursing participants had successfully used acuity data to secure additional staff. However some nursing participants felt that the measurement tools were inadequate and could not accurately capture all the components which contributed to a patient's acuity level. Furthermore nursing participants displayed some antipathy towards senior management's attitude to them collecting this data.

"Yeah. So there's been a fight with them about that because they tell it's (a) and you say no it's not it's (b) and they go no, no it's not 
it's (a) and it's like I just don't want to fight with anybody any more" (FGNursesSurgx2H2)

Senior management participants described acuity measurement as ineffective and inaccurate and therefore not helpful in terms of ensuring staffing ratios were appropriate.

A further factor believed to affect the care of acutely unwell patients was restrictions in practice regarding specific aspects of care. Medical participants described their frustration at not being able to institute care such as Continuous Positive Airway Pressure (CPAP) or inotropic therapy on particular wards. These specific treatment therapies could only be provided in high dependency units (HDU) which were not always readily accessible. Therefore treatment options for patients were limited by the patient staying on the ward. Finally, nursing participants felt that the requirement for documentation of nursing care had increased over time to the point that it took them away from delivering patient care.

Access issues were associated with referral processes and barriers between specialties. Participants described difficult and time-consuming referral processes which put acutely unwell ward patients at risk of further deterioration.

"You have people who are process driven and irrespective of what's happening the process has to be followed and sometimes unless you have gone through all the steps it's really difficult for them to see the outcome is going to be the same" (AHSNurs1H2)

Participants used expressions such as "being pushy", and "jumping through hoops" in order to get patients seen by other specialties even if the patient was acutely unwell. This was believed to be due to territorialism and was affected by relationships between doctors from specific specialties.

"I guess I actually do wonder sometimes whether it's to do with who knows who. I don't know whether it's like that with all other hospitals around the place but this is very much hospital who knows who and people have worked with other people and know them quite well" (FGNursesSurgx2H2)

Nursing and medical participants also described situations where specialty doctors focused on specific specialty-related patient problems rather than seeing the patient as a whole. 
"sometimes each person is so focused in their own area that it does distract you know the fact that there's another system now getting involved for whatever reason and that sometimes you know we have to say to them yep it may not be a surgical problem but look this patient seems to be having a myocardial infarction here and you know we need to get cardiology involved" (AHSNurs2H2)

Not attending to the patient as a whole led to patients having specific health issues which were either not recognised or not treated. Nursing and medical participants felt that lack of attention directly affected the care of acutely unwell ward patients. Table 13 summarises the key factors to emerge from the theme of organisational systems and processes of care.

Table 13. Factors to emerge from the theme of organisational systems and processes of care

\section{Theme Factors}

Organisational systems and processes Service initiatives of care Organisational policies Access

\subsubsection{Culture and environment}

Ward and organisational culture, nurses' cultural background, and interactions with families were all factors within the theme of culture and environment. Ward culture was associated with what were acceptable attitudes and behaviours within a particular ward environment. Participants talked of supportive ward cultures, where staff felt comfortable asking for help, were encouraged to prioritise deteriorating patients over more routine patient care and where staff engaged with each other socially. In contrast, unfavourable ward cultures were where education and training of nursing staff was not a priority, where nurses were not encouraged to up-skill and where nurses were afraid to challenge particular attitudes and behaviours. Nursing participants described disorganised ward areas where questioning decisions regarding patient care was not encouraged. This disorganised and unquestioning approach was viewed as an acceptable part of the culture of some wards, which nursing and managerial participants believed was detrimental to the care of acutely unwell ward patients. 
Organisational culture encompassed the attitudes and beliefs promoted by each organisation. Executive management participants described a culture which was open and transparent, particularly regarding reporting of serious or sentinel events. The executive management participants believed that their organisational culture promoted teamwork where poor behaviour would not be tolerated.

"But what we want is a commitment I think, I'm pretty sure we need a commitment from the chief medical advisor down that poor behaviour of consultants and of registrars and of house surgeons will all be addressed (EMTH2)

However senior managers described some aspects of the organisational culture as out of synch with the reality of working on the wards. This particularly related to nursing workloads and staffing levels and the level of clinical risk within the organisation. When these managers relayed their concerns to their seniors they perceived the responses to be unhelpful.

"They don't often come up with anything particularly useful other than oh well they'll manage"

Okay. So is there an understanding of the clinical risk that might be out there? (SQ)

I don't know if it's understood and ignored or I think actually they just choose to ignore it and they deal with it as a one on one thing. I think they actually just choose to ignore it. It's too difficult to cope" (SenManMedH2)

According to management participants, a supportive organisational culture which focused on patient safety was determined through the provision of sufficient processes and resources to support the care of acutely unwell ward patients. Not surprisingly, the majority of the executive management participants believed that their organisation was very focused on improving the care of this patient group through the introduction of support services such as CCOS and EWS. Despite executive management's perceptions of a supportive culture, senior managers were less convinced that the organisational culture was entirely supportive. Their reservations stemmed from being subject to laborious processes regarding recruitment which left wards short staffed for what they considered overly long periods, which they believed directly impacted on safe and effective care. In 
addition they perceived that service initiatives were introduced without proper preparation, ongoing support, and evaluation.

The cultural background of the nurses caring for acutely unwell ward patients was believed to be a factor which impaired care. Nursing participants believed that nurses trained in the Philippines were less likely to complain about their workloads, were less likely to question the decisions made by doctors and senior nurses and could be more task focused than New Zealand trained nurses. The nurse's cultural background could also affect their interactions with families. Nursing participants believed that overseas-trained nurses could be intimidated by the presence of some families and shied away from direct contact with patients' relatives due to a perceived lack of knowledge of Maori culture or because of language difficulties. Finally, ward layout was believed to affect the care of acutely unwell ward patients because nurses were unable to visualise patients easily due to the cubicle layout. Wards were seen as cramped and 'very nurse inefficient', which again impacted on the time available to care for this patient group. Table 14 summarises the factors to emerge from the theme of culture and environment.

Table 14. Factors to emerge from the theme of culture and environment

\section{Theme}

Culture and environment

\section{Factors}

Ward culture

Organisational culture

Nurses' cultural background

Interactions' with families

Ward layout

\subsection{Education}

The final domain to emerge from the data was education. Within this domain two themes became apparent. These were skills and knowledge deficit, and opportunities for learning.

\subsubsection{Skills and knowledge deficit}

Within this theme participants perceived inadequate undergraduate training of both doctors and nurses as a key factor which influenced the care of acutely unwell ward patients. Within nursing, executive management participants felt that 
the change in training which shifted the emphasis to a degree-style university education had produced graduate nurses who were not adequately prepared to care for patients, especially acutely unwell patients. Executive and senior management participants expressed the view that this was predominantly due to lack of clinical exposure during their training.

"I think you know if you compare what we have now to the traditional old model of hospital-based training and things like that people did get more exposure to more acutely unwell people. And that's a fact. And so maybe this has been a reflection of the change in which we've undertaken the education for nurses. Yeah I think that might be a factor" (EMTH1)

Lack of clinical exposure during undergraduate training was also raised as an issue in medical training. Executive and senior management participants felt that there had been an increase in academic input at the expense of clinical placements, leaving newly graduated doctors unprepared for the challenging clinical environment. This was especially relevant since these doctors were most likely to encounter acutely unwell ward patients after hours, when there was little senior support to assist them.

"So I think in ten years we're also seeing a slight decline in the level of experience of the graduates that are coming yet these are the people who are there after hours and actually having to deal with things" (SDH1)

These shortfalls in training were recognised by the executive and senior management participants, who described the organisational investment required in terms of training and education to meet these deficits. Skills and knowledge deficits highlighted by nursing and executive and senior management participants were mainly regarding nurses' and doctors' patient assessment skills and the ability to accurately record and interpret vital signs. Participants believed that nurses relied heavily on machinery to take vital signs and because of this some important nursing skills had been lost.

"We're trying to work very strongly that they look at the patient, they touch the patient and look at the skin tone, at the feel, look at the pulse you know with your hand not a machine. Unfortunately we seem to have a generation of nurses that rely on electrical machines 
rather than their own endobedogram. They don't look at what their eyes are telling them" (CNEMedH1)

Participants in all roles suggested that a lack of assessment skills led to delays in recognising when a ward patient was becoming unwell. Participant doctors described occasions where they had been informed at a very late stage that patients had deteriorated. Explanations for this were that nurses were unable to interpret vital signs appropriately, were unable to determine the seriousness of the situation, had poor basic physiological knowledge and that they 'normalised' abnormal recordings, i.e. the patient's blood pressure has been low for two days therefore this must be normal for that patient. Participants also noted that even when nurses recognised vital signs were abnormal they did not escalate care appropriately.

"You've got one elevated parameter and then there's a big gap it hasn't been checked again for two hours or four hours or something where it should have been rechecked an hour later or half an hour later depending on what the parameter was" (CNESurgH2)

By the time nursing staff got around to checking the abnormal parameter the patient had deteriorated further. Some participants suggested that nurses may have intended to recheck the abnormal parameter more frequently but had been delayed through caring for other patients. Table 15 summarises the factors to emerge from the theme of skills and knowledge deficit.

Table 15. Factors to emerge from the theme of skills and knowledge deficit

\begin{tabular}{|l|l|}
\hline Theme & Factors \\
\hline Skills and knowledge deficit & Workforce preparation \\
& Assessment skills \\
& Prioritising \\
& Escalating care \\
& Using machinery \\
\hline
\end{tabular}

\subsubsection{Opportunities for learning}

Within the theme of opportunities for learning, bedside learning and receptiveness to education were important factors. Senior management participants discussed the need for ongoing education which occurred at the 
bedside. These participants suggested the ideal situation for learning about caring for an acutely unwell patient was when a more senior nurse stood by the side of a junior nurse coaching her through the care required. However some medical and executive management participants felt that despite organising education sessions relevant to caring for acutely unwell patients, some staff were not interested or motivated to learn. Table 16 summarises the key factors to emerge from the theme of opportunities for learning.

Table 16. Factors to emerge from the theme of opportunities for learning

\begin{tabular}{|l|l|}
\hline Theme & Factors \\
\hline Opportunities for learning & $\begin{array}{l}\text { Bedside learning } \\
\text { Receptiveness to education and training }\end{array}$ \\
\hline
\end{tabular}

\subsection{Summary}

Using the three domains of healthcare workforce, education, and organisation, individual data sources from nurses, doctors, and managerial participants at all levels were coded. Domains, themes, and factors which emerged are presented as Table 17.

Table 17. Domains, themes, and factors

\begin{tabular}{|l|l|}
\hline \multicolumn{2}{|c|}{ Healthcare workforce } \\
& Having time and coping \\
& Task focus \\
& Allocation \\
& Nurse to patient ratio \\
& Skill mix \\
\hline Teamwork & Relationships \\
& Support \\
& Credibility and Trust \\
& Clinical experience \\
& Being confident \\
\hline Communication & Realthcare workforce \\
\hline Leadership, accountability and roles & Responding \\
& Breakdown in communication \\
\hline & Being accountable \\
& Seeing the bigger picture \\
& Roles of doctors and nurses \\
\hline
\end{tabular}


Table 17. (continued). Domains, themes, and factors

\begin{tabular}{|l|l|}
\hline \multicolumn{2}{|c|}{ Organisation } \\
\hline Organisation of $\mathbf{2 4 / 7}$ services & $\begin{array}{l}\text { Support } \\
\text { Continuity of care }\end{array}$ \\
\hline $\begin{array}{l}\text { Organisational systems and processes } \\
\text { of care }\end{array}$ & $\begin{array}{l}\text { Service initiatives } \\
\text { Organisational policies } \\
\text { Access }\end{array}$ \\
\hline Culture and environment & Ward culture \\
& Organisational culture \\
& Nurses' cultural background \\
& Interactions with families \\
& Ward layout \\
\hline Skills and knowledge deficit & Education \\
\hline Opportunities for learning & Workforce preparation \\
& Assessment skills \\
& Prioritising \\
& Escalating care \\
& Using machinery \\
\hline & Bedside learning \\
& Receptiveness to education and training \\
\hline
\end{tabular}

Domains, themes, and factors in Table 17 are represented in order of weight of data, e.g. factors related to healthcare workforce were most discussed by participants hence it features first in Table 17 and so forth. This table represents the analytical framework used for the individual cases. The analytical framework was used to analyse data from the individual cases and hospitals. This within-case and within-hospital analysis is presented as Chapter Five. 


\section{CHAPTER 5: ANALYSIS OF THE CASES}

\subsection{Introduction}

Chapter 4 provided an initial analysis of data collected in interviews and focus groups with nurses, doctors, and managerial participants. These data were used to formulate the analytical framework which is presented as Table 17. This chapter now presents the within-hospital analysis and the within-case analysis.

In order to provide context for the cases a brief description of the organisation (Hospital 1, Hospital 2) in which the wards are located is provided. To facilitate the ethical requirements of this study some identifying features of the hospitals are either presented as ranges (e.g. number of beds, number of staff) or specific details have been omitted.

Following the description of each hospital, a within-hospital analysis of each hospital is presented. This within-hospital analysis represents the reanalysis of interview and focus group data (using the analytical framework presented as Table 17) of research participants who worked across the organisation. Research participants who worked across the organisation are from three separate groups: senior or executive managers; nursing participants who worked in support roles across the organisation; and the house surgeons who worked in Hospital 1. In addition relevant documentation from each organisation was reviewed and used to augment data from the interviews and focus groups. The analysis of this hospitalwide data provides a context in which to ground the individual case data. Furthermore, using data from participants who worked across the organisation ensured a thorough exploration of each case.

Following the presentation of each within-hospital analyses, the within-case analyses are presented. According to Stake (1995) sufficient detail should be provided to allow the reader a 'vicarious experience' (p. 63) of the case. Therefore there is a description of each ward which includes some detail describing the ward layout and some facts about the ward personnel. Again, identifying features of the wards have been omitted to comply with ethical requirements. Following the description of each ward, the analysis of each case is presented. This is derived 
from re-examining the interview and focus group data using the analytical framework. Only data from participants who worked on each specific ward were included in the within-case analysis. In addition pertinent documentation from each ward was reviewed and used to provide a description of the ward and to augment data from the interviews and focus groups.

Participants discussed many issues during their participation in this study. However, for the purpose of this chapter, only the key themes which emerged from each individual case are presented. Key themes are defined as those themes most discussed by participants. The analytical framework (Table 17) presents the domains, themes, and factors in a particular order. However, in this chapter the domains, themes, and factors are presented according to the weight of the data as identified by participants from a specific case. Those most discussed by participants feature first. As a consequence this may not match the presentation of the order of the domains, themes, and factors presented in Table 17.

\subsection{HOSPITAL 1}

Hospital 1 was located in the North Island of New Zealand. At the time of the study it had between 200 and 300 acute beds and served a population between 100,000 and 200,000 people from the local area. The hospital provided secondary and tertiary, medical and surgical hospital services alongside community-based health care. The hospital employed between 1500 and 2000 staff. The hospital also provided specialist regional services in rheumatology, plastic and maxillofacial surgery, public health, and cardiac magnetic resonance imaging (MRI). In addition the hospital provided general medical, cardiology, orthopaedic, general surgical, paediatric, obstetric and gynaecological, rehabilitation, and mental health services.

\subsection{Hospital 1: Within-hospital analysis}

In order to provide contextual detail in Hospital 1, senior and executive management, junior medical staff, and the manager of the critical care outreach service (CCOS) were interviewed or took part in focus groups. The titles and specific numbers of these participants are illustrated in Table 18. 
Table 18. Organisational participants - Hospital 1

House Surgeons x 9 (Focus group - a mix from surgery and medicine)(FGHSx9H1) Senior Manager Specialist Service Hospital 1 (SenManSPH1)

Senior Nurse Medicine and Senior Nurse Surgery (FGSNx2H1)

Medical Directorate Manager (SenManMedH1)

Consultant Physician (SDH1)(nominated by the Chief Medical Advisor)

Director of Nursing (EMT1)

Chief Operating Officer (EMTH1)

(Letters in parenthesis are code names from participant transcripts)

Within this group of participants there was one large focus group of nine participants. These house surgeons worked on either the medical or surgical ward within Hospital 1, which is why they were eligible to participate in the study. However, during the transcription of the house surgeons' focus group it was impossible to distinguish between participants and therefore to discern whether the comment was about a particular ward. Therefore the decision was made to use the data from this focus group as generic information regarding the organisation and not specifically about one ward.

All organisational participants were asked to discuss organisational factors which affected care of acutely unwell ward patients. In addition to interview and focus group data, documentation pertaining to the care of acutely unwell ward patients was reviewed. Table 19 lists the documents reviewed as part of the organisational analysis for Hospital 1.

Table 19. Documents reviewed for Hospital 1

Patient Safety Leadership Group Terms of Reference

Event Reporting and Management Policy

Using your Consultant - Guidelines for Registrars

Guidelines for reporting concerns if a patient's condition deteriorates

Risk Management policy

Nurses and Midwifery Training Opportunities Training Manual

All three domains of healthcare workforce, organisation, and education emerged from the interview and focus group data from Hospital 1. Of the three, the main domain was organisation. The three themes to emerge from organisation were culture and environment, organisational systems and processes, and organisation of $24 / 7$ services. These will be discussed first, followed by the key 
themes from healthcare workforce (communication; leadership) and education (workforce preparation). All themes will be discussed according to the weight of the data.

\subsubsection{Culture and environment}

Participants believed that part of the organisation's culture was to constantly seek ways in which it could improve care to acutely unwell ward patients. According to executive management participants the organisational culture was based on specific quality principles which included: promoting a strong collective leadership culture; using empirical data to influence care at the bedside; and having an open and transparent system for dealing with serious and sentinel events. Executive management participants suggested that when these quality principles influenced organisational systems and processes they positively affected care of acutely unwell ward patients. Quality initiatives had been introduced to include the implementation of a Quality Report, which was a list of key performance indicators (KPIs) that each ward reported on monthly. The KPIs were divided into five domains: effectiveness; efficiency; patient safety; experience; and, finally, education. Each domain was further divided into specific, measurable KPIs. For example, in the efficiency domain casual and agency nurse usage, sickness and turnover were specific indicators. Indicators in the patient safety domain were Early Warning Score (EWS) uptake and patient falls. For each indicator there was an organisationally set target. The quality reports were displayed on a notice board in each ward to demonstrate progress towards meeting these targets.

Executive management participants described the importance of building in quality reporting systems and using data effectively to support service delivery changes.

"So without the ability to use things like nursing sort of indicators or the KPIs you don't really have the data to support what you're trying to achieve so if you get people to focus on it and it's patchy we haven't achieved it totally. There's a lot of work to do but it's led people into focusing on those sorts of things. So people now do talk about what are the KPIs or what are we looking at, what are we trying to achieve here" (EMTH1) 
Although executive management participants believed in the importance of KPIs, there was an appreciation that using empirical data to influence care at the bedside would take time.

According to a member of the EMT the culture of the organisation placed the best interests of the patient at the centre of any decision-making processes regarding policy or processes. This EMT participant believed that this should be supported by establishing good team relationships facilitated by open and honest communication, especially in relation to the open disclosure policy. However, executive management participants were realistic regarding differing attitudes to open disclosure and that developing that sort of culture took time.

"We've tried to engender that as a culture as well. So an openness really and non punitive. So if an event occurs it's not a punitive process. Yeah so I mean, there's a lot of work to do there. Education wise and just getting people comfortable with saying look we made a mistake here and it was because of such and such we did that. And, then when you really look at it nobody comes to work to make a mistake. So if we can sort of foster that it would be all the better for patients and staff" (EMTH1)

At the time of the study, to further support the organisation's commitment to patient safety Hospital 1 had a patient safety leadership group which the COO, DoN, and CMA all attended, with other representatives from allied health and quality services. The purpose of these meetings was to review serious and sentinel events which occurred in the organisation. The meetings took place weekly and were minuted. The terms of reference for the meetings included the provision of leadership in the promotion of a patient safety culture, ensuring open disclosure. They also included the provision of a forum where staff discussed and resolved issues of clinical quality, ethical decision making, medico-legal and general patient safety issues. The minutes from these meetings were confidential and were circulated only to attendees.

Within Hospital 1 the medical floor had undergone significant changes after the occurrence of a sentinel event. The sentinel event raised several issues, which included communication, teamwork, managing complex patients, and case management. In addition there were issues with communication between the 
clinical nurse managers and the ward nurses. Finally, the standard of care being delivered on the ward had been questioned. When discussing the medical ward, executive management participants described a ward culture where, historically, questioning care decisions was not supported, where staff education had not been optimal and where role modelling had been non-existent. Leadership had not been strong and the ward had appeared chaotic.

"Sometimes you come down to the medical floor and it maybe it's a question of size, but sometimes you have this feeling of chaos and where is the leadership and the charge. And I think that's been changing" (SDH1)

This prompted the organisation to strengthen the ward leadership structure as well as making sure that the Clinical Nurse Managers (CNM) were clear about what was expected from them. Furthermore, a new model of care and a 'back to basics' campaign were implemented. Management participants believed that all these changes were starting to have an effect.

"Medical is doing very well. We're getting lots of very positive feedback, positive comments from patients but it is slow to change the culture and there have been some cultural issues in the medical area. It's not everybody's cup of tea. It's heavy. It's hard work. It's busy. It's also staffing ratio probably hasn't been as good as a lot of areas" (SenManMedH1)

Management participants recognised that changes would take time, but believed that the organisation recognised that further input was required and they were committed to that further input. There was a perception that the medical ward nurses had taken the burden of the changes that had been implemented with minimal effect on the ward medical staff.

"Why does it look like we're the only ones responsible here for what happened to this patient because they certainly weren't at all. But it just sort of felt that all the onus did fall on them and they've sort of been asked to make some major changes and very little changes appear to have happened within the medical staff"(FGSNx2H1)

For example, ward rounds on the medical ward were highlighted by several management participants as being challenging.

"I don't have any outcome on that, any thoughts on what the outcome would be in terms of any particular change, but what I'm 
suggesting is that we should challenge the status quo. That we should be saying is there a better way, we should always be asking ourselves the question is this the best way of working for the patient" (EMTH1)

Participants believed that ward rounds were time-inefficient and an example of an 'old system which may not be sustainable into the future'. However, there were no plans to change how they were currently organised.

Some senior and executive management participants believed that the surgical and medical wards had different ward cultures. There was a view that the surgical ward had a core of senior nurses who 'dominated' and did not accept new initiatives as readily as more junior nurses. Another difference in ward culture highlighted by participants was that the need to measure patient acuity was deeply embedded within the surgical ward culture unlike the medical ward, where there was no commitment to measuring or using acuity data.

\subsubsection{Organisational systems and processes of care}

Hospital 1 had implemented several new service initiatives and education programmes to promote patient safety. According to participants, the rationale for the implementation of these new initiatives was based on serious and sentinel events which had occurred on the wards, the junior doctors' strike of 2008, and a perceived degree of clinical risk after hours.

These new service initiatives and education programmes included the implementation of an Early Warning Score (EWS), a Critical Care Outreach Service (CCOS), the Acute Life Events Recognition and Training programme (ALERT) and a back-to-basics campaign for the medical floor. According to management participants, EWS was a good initiative for 'watching' patients and also for nursing staff to bring patients to the attention of medical staff in a consistent way. Conversely, the medical participants believed that at times nursing staff simply 'totted up the numbers' and did not think beyond the score, which rendered the scoring system ineffective and adversely affected the care of acutely unwell ward patients. However, the organisation was committed to using the score and 
compliance with EWS was a key performance indicator on each ward area's quality report.

The CCOS covered the ward areas on all afternoon shifts, seven days a week. The purpose of the service, according to participants, was primarily to manage risk after hours. This service was described by one senior management participant as a 'band aid' solution to support a rapid turnover of staff, support the junior doctors after hours and to support ward staff to care for patients discharged from ICU or being admitted from the emergency department (ED). There had been a history of mistrust regarding the ability of nursing staff to manage particular patients on the wards.

"The ward area should be able to manage these patients with support and previously there was a lot of resistance from clinicians when ICU consultants, anaesthetic consultant wanted to discharge patients from ICU because they didn't feel that they could manage them in the ward. And to be fair to the clinicians their historical perspective of how the nurses in some areas managed very sick patients didn't give them any feeling of security" (SenManMedH1)

In order to augment care for acutely unwell ward patients the organisation had recently committed to the development of a new High Dependency Unit (HDU) on the medical ward. The purpose of the HDU was to cohort all the acutely unwell patients within one area of the ward. Nurse patient ratios would be altered to ensure that nursing staff had a lighter patient load in order to deliver the level of care these patients required. There were differing views regarding how the new HDU was to be implemented.

"[NAME REMOVED] originally didn't want to do it until she had everything up front. I want the money to do this. I want the staff. In healthcare in the current financial constraint that doesn't happen and my theory has always been, look just do it. If you just start to do it with what you've got, you start with one patient in that area and then you bring in two patients. You up skill your nurses as you go. You will get there faster. In fact you won't get there at all if you want somebody to hand it to you on a plate"(SenManMedH1)

Some funding had been allocated to the development of the new unit but the staffing budget was to remain the same. The ward staff from ward A were to be trained in HDU care and used to staff the new unit. The medical directorate 
manager believed that this was a viable option and that the CNM needed to be resourceful and redesign how staff delivered care.

"But seeing as they're looking after those patients anyway it's about redesigning what they do. So if you're pulling the sicker ones to one area, yes they might need a one to two ratio but consequently the ones that are left out in the other area would be a lesser ratio" (SenManMedH1)

Without the willingness to 'just do it' the services that were required to care for acutely unwell patients on the medical floor would not be implemented.

The main organisational policy discussed by participants was the management of outliers. According to the medical directorate manager there could be up to 25 medical patients being looked after in surgical wards at any given time. Participants believed that medical outliers were at risk. Surgical nurses were not as knowledgeable as medical nurses in all aspects of caring for medical patients. The organisation had recently advised all CNMs that if the patient was admitted under a specific speciality they still remained that speciality's responsibility in terms of care, regardless of which ward they were on. Staff had also been advised to keep sicker medical patients within the medical wards and move the patients who were ready for discharge to the surgical floor.

\subsubsection{Organisation of $24 / 7$ care}

The organisation of $24 / 7$ services and the lack of support out of hours was described by participants as an ongoing concern in relation to the care of acutely unwell ward patients. The house surgeons' group voiced concern regarding the difference in the way patients were managed outside of normal working hours.

"Just speaking generally then I think most of the stories you're going to get when you talk to people will be all after hours and all will be in the weekends, and all will be in the holidays. So obviously there's a clear cut difference between the way people are managed after hours and the way they're managed just during normal working hours" (FGHSX9H1)

Their concerns were focused on continuity of patient care and the difficulties associated with shift work. They believed there were often situations when no one 
'on call' knew the patient. As a consequence they relied heavily on the nursing staff to provide adequate information to help them manage the patients appropriately. The organisation recognised that the level of expertise and numbers of staff diminished after hours. The medical directorate manager believed that having a 'hospital at night' service to further ameliorate risk would be beneficial.

"Having a formalised hospital after hours team which links in the registrar, the ICU registrars with the extra ICU registrar position in there, would make a big difference"(SenManMedH1)

Following the referral of a case to the Health and Disability Commissioner (HDC) a lack of expertise regarding airway management was highlighted as an issue. The Health and Disability Commissioner's role is to ensure that the rights of consumers are upheld, including making sure that complaints about health or disability services providers are taken care of fairly and efficiently. Following this review by the HDC the hospital was committed to developing a more team-based approach to the overnight management of acutely unwell ward patients.

\subsubsection{Communication}

Communication emerged from the domain of healthcare workforce as a key theme. At the time of the study Hospital 1 used a computerised communication system (CCS) which operated from 1600 until 0800 on weekdays and from 1600 on Friday until 0800 Monday. The purpose of the system was to improve communication after hours. Nurses 'put up' or listed jobs on the system which were prioritised in a traffic light system; green for non urgent requests; amber for semi urgent requests and red for urgent requests. Although aimed at the house surgeons, all levels of medical and nursing staff were able to access the CCS and could also view the requests from any computer terminal in the hospital. According to medical participants, the system had reduced the number of times doctors were paged; however, the medical participants were concerned at times about the nurses' ability to appropriately prioritise jobs on the system. They relayed incidents when nurses had listed patients on the system that were coded as 'red' and therefore allegedly very urgent. 
"I've had a couple of urgent situations after hours where I really should have been called by the nurse, not from [ACRONYM REMOVED] and we had even stated, well the medical registrar and me had stated, that if these things happen you should call us but they didn't they put it on [ACRONYM REMOVED] and the person's systolic blood pressure was dropping. Like first it was 70 and then it was 60" (FGHSX9H1)

Guidelines for the system state that if a 'red' request is put onto the system then it should also be accompanied by a phone call to the house surgeons or registrar to relay the urgency of the request. This is because the house surgeons can know about the urgent patient review on the system only if they are accessing and checking CCS regularly. If they were busy seeing patients for prolonged periods their ability to check the system is affected. Furthermore, medical participants believed the system was only as good as the information entered onto it and were frustrated by the lack of detail that nursing staff provided. This directly affected the house surgeons' ability to triage requests, organise their workload and review acutely unwell ward patients in a timely fashion. In addition, requests for urgent reviews which the medical participants considered inappropriate also led to a breakdown in communication.

"Because to be fair if they ring us with stuff that's really inappropriate sometimes you, if you're not stressed you might respond and actually educate them but you're more likely to be like 'that's ridiculous'. In fact I wouldn't quite use those words but you know it comes across, if that's what you're thinking it's going to come across in your tone or shortness of how you answer them and it just creates barriers to proper referrals because they're going to be more nervous about paging" (FGHSx9H1)

The medical participants also became irritated when they had been requested to see a patient urgently and arrived on the ward and could not find either the nurse looking after the patient or the patient's notes and charts. These participants felt that this did not promote good working relationships between medical and nursing staff and they also believed the nursing staff should be able to communicate at the same standard as they communicated.

Another factor which led to poor communication discussed by participants was inadequate handover procedures or a lack of understanding regarding the 
purpose of handover. Medical participants believed that because there were no formal handovers between doctors, information about patients often 'slipped through the gaps'. Nursing and senior management participants stated they believed that staff did not understand that handover was not just about the transfer of information but its purpose was also to hand over responsibility and accountability for the patient.

The SBAR communication framework had been rolled out across the organisation as a way of communicating. Some wards had trialled SBAR as a way of providing a 'framework for what was important' in the handover process. However nurses had raised concerns about the length of time handover was taking.

"They identified initially that they were having problems with the length of time the handover was taking and [what] that does seem to be a primary concern in all areas. Let's make handover as quick as we possibly can" (FGSNx2H1)

In the medical ward where a Dictaphone was used as part of the handover process the only guidelines nurses were given were regarding the time the nurse had to handover each patient. For some participants using the Dictaphone was perceived as inadequate if the purpose of handover was the transfer of responsibility and accountability.

"but I don't think we'll ever shift them away from Dictaphone but I'm sure there's many reasons you could find why you should. It's not necessarily a good idea" (FGSNx2H1)

However there were no imminent plans to change the handover process on the medical ward.

\subsubsection{Leadership, accountability and roles}

Another key theme to emerge from the domain of healthcare workforce was leadership. Strengthening the leadership roles at ward level had been a recent organisational focus. However this had been restricted to the CNMs and Associate Charge Nurse Managers (ACNM), and not the nurses who coordinated shifts after hours. Some participants believed that the role of the coordinator was complicated in relation to the care of acutely unwell ward patients. 
"And that's the expectation here. Oh yeah you have your full workload and probably sickest patients but also you know are the shift coordinator and I mean that's just not possible in you know in a really busy shift because you'll also be fielding queries from after hours managers about bed availability and you know doing all those sorts of things which detracts" (FGSNx2H1).

Some management participants believed that nurses who assumed the role of coordinator should either have a lighter patient load or no patient load at all so that they had sufficient time to have an overview of what was happening on the ward. These participants also believed that the assumption that a senior nurse had good leadership skills and was therefore able to lead a shift as a coordinator was flawed. The organisation did not provide specific training for coordinators, although listed in the hospital 'Nurses and Midwifery Training Opportunities' manual was a training day entitled 'Direction and Delegation'. At the time of this study this one-day study day provided content on leadership and communication, scopes of practice, and misconceptions of direction and delegation.

\subsubsection{Teamwork}

The final theme to emerge from the domain of healthcare workforce was teamwork. Within the theme of teamwork fostering good relationships between team members was considered an important aspect of the culture of the organisation according to senior and executive management participants from Hospital 1.

"I guess the big elements that support that multidisciplinary teamwork, relationships. Having really strong relationships between clinicians and between management and clinicians so that we can focus on systems and process to improve quality of care acutely or electively or whatever but that we are very process orientated" (EMTH1)

Executive and senior management participants believed that relationships between nursing and medical staff were on the whole good which contributed to effective team working and supported junior staff in their care of acutely unwell ward patients. However this was not a viewpoint held by all participants. Medical 
participants described difficulties with teamwork which could be dependent upon other team members' personality and workload.

"You're meant to call the surgeon, the surgical registrar first because it's their patient and then they say 'oh actually this is a medical issue' so then you have to convince the medical registrar to come down and see the surgical patient (FGHSx9H1)

Finally some medical participants felt that the availability of support impacted on teamwork especially afterhours.

"The person coordinating the care will be someone more senior [in the Emergency department] but we don't really have that in the general surgical and medical wards because virtually the person in charge of the care is the house surgeons" (FGHSx9H1)

Medical participants described appreciating the opportunity to discuss perceived patient problems with seniors in a more formal handover setting at night as a means of fostering effective teamwork.

\subsubsection{Skills and knowledge deficit}

The key factor to emerge from the domain of education and the theme of skills and knowledge deficit was workforce preparation. Participants felt that undergraduate training of nurses and doctors was not preparing them adequately for the current healthcare environment. Executive and senior management participants believed this was due either to a lack of clinical exposure or to inappropriate clinical placements as students. Not getting the appropriate exposure led to new graduate nurses and doctors having insufficient skills to care for acutely unwell ward patients.

"I think you know if you compare what we have now to the traditional old model of hospital-based training and things like that people did get more exposure to more acutely unwell people. And that's a fact. And so maybe this has been a reflection of the change in which we've undertaken the education for nurses. Yeah I think that might be a factor" (EMTH1)

This meant that the organisation had to fill this perceived gap by providing additional training in the shape of the ALERT course and the back-to-basics campaign. 


\subsubsection{Summary}

All three domains of healthcare workforce, organisation, and education, emerged from the interview and focus group data for Hospital 1. Table 20 presents what the participants believed were factors most affecting care of acutely unwell ward patients in this hospital.

Table 20. Domains, themes, and factors Hospital 1

\begin{tabular}{|l|l|}
\hline \multicolumn{2}{|c|}{ Organisation } \\
\hline Organisation of 24/7 services & $\begin{array}{l}\text { Support } \\
\text { Continuity of care }\end{array}$ \\
\hline $\begin{array}{l}\text { Organisational systems and processes } \\
\text { of care }\end{array}$ & $\begin{array}{l}\text { Service initiatives } \\
\text { Organisational policies }\end{array}$ \\
\hline Culture and environment & $\begin{array}{l}\text { Ward culture } \\
\text { Organisational culture }\end{array}$ \\
\hline \multicolumn{2}{|c|}{ Healthcare workforce } \\
\hline Teamwork & Relationships \\
\hline Communication & $\begin{array}{l}\text { Responding } \\
\text { Breakdown in communication }\end{array}$ \\
\hline Leadership, accountability and roles & Leadership roles \\
\hline \multicolumn{2}{|c|}{ Education } \\
\hline Skills and knowledge deficit & Workforce preparation \\
\hline
\end{tabular}

\subsection{MEDICAL WARD A}

\subsubsection{Setting the scene}

At the time this study was conducted Ward A was a general medical ward with 27 beds. There were four admitting consultants who were general physicians.

Typical admissions consisted of patients presenting with gastro-intestinal, respiratory, cardiac, and neurological complaints. The ward had a specialty focus in that patients requiring BiPAP (a form of non invasive ventilation) were admitted here. The ward leadership team consisted of a Clinical Nurse Manager (CNM) 1.0 Full Time Equivalent (FTE), Associate Clinical Nurse Manager (ACNM) 1.0 FTE and a Clinical Nurse Educator (CNE) 0.5 FTE.

The ward consisted of a mixture of four-bedded bays and single rooms. One of the single rooms was slightly larger than the others and was used for patients who had large numbers of visitors, especially in end-of-life situations. On entering the ward there was a large open office for health care staff where patient notes were kept. There were several computer terminals located here. There was a desk 
at the front of this area for the ward clerk and this was the first point of contact for people entering the ward. There was a day room at the end of the ward for patients and visitors. Adjacent to this was a large office which was shared by the CNM, the CNE, the patient discharge coordinator, and the clinical nurse specialist for case management.

The staff room was located in the middle of the ward. This was a small room with no natural light. There were dining chairs, a dining table and one two-seater sofa. There were three notice boards dedicated to staff training and professional development and quality improvements. For the most part the notices on these boards were in date. There was a phone in this room but staff did not answer it when it rang as the calls went through to the reception area where the ward clerk answered them. The patient call bells could be heard in the staff room and there was a dot matrix indicator notice board which displayed the patient bed number of the patient who had activated the call bell. There was a Dictaphone where staff recorded their patient details for handover.

\section{Ward Personnel}

At the time of the study the nursing establishment for the ward was 26.9 FTEs which equated to 28 actual staff members indicating that the majority of staff were full time. The current staff numbers were 20.4 FTE showing a vacancy rate of 6.5 FTE. The nursing roster was split into three sections; senior, intermediate and junior. Within these sections the CNM identified 19 members of staff as senior, four as intermediate, and five as junior. Staff were determined as senior by time spent on the ward and not by their position on the Professional Development Recognition Programme (PDRP). The PDRP is a clinically-focused competencybased programme for nurses. It is a national programme, endorsed by the Nursing Council of New Zealand and the New Zealand Nurses Organisation, used to assess continuing competence and level of practice where appropriate.

The ACNM coordinated the ward morning shifts Monday to Friday. When there was no ACNM, available senior staff coordinated shifts. When the ward was fully staffed the coordinators did not carry a patient load. This most commonly occurred on afternoon shifts and at weekends. On every night shift the most senior 
of the rostered nurses coordinated the shift in addition to taking a patient load. The ward did not provide any formal training for coordinators.

Rostering was done via a semi self rostering model. Blank roster forms were put up in the staff room and the staff pencilled in the shifts that they wanted to work. The CNM altered the requested shifts to ensure that the ward was adequately covered as required. If staff worked part time they could put in 'extra shifts' to cover the ward. This was not actively encouraged in full time staff, although staff had done 'double shifts' (usually afternoon and night shifts) due to unforeseen sickness.

There were two ward clerks during the week. They covered the ward during the hours of $0730-2000$. At the weekend they worked from $0730-1630$. Their role was to meet and greet visitors to the ward, collate patient notes, answer the phones and book patient appointments.

\section{Patient Acuity Tool}

The ward had a patient acuity tool but this was rarely used as the ward staff believed it did not necessarily reflect patient acuity accurately. No other form of patient acuity measure was used in its place.

\subsection{Medical ward A: Within-case analysis}

The main issues to emerge from participants working on this ward were from the domain of healthcare workforce' specifically factors associated with the themes of workload, teamwork, and communication. Participants who took part in interviews and focus groups from this ward are listed below in Table 21.

\section{Table 21. Participants from Medical ward A}

Medical Nurse B (NursBMedH1)

Medical Nurses x6 (FGNursesMedx6H1)

Clinical Nurse Educator (CNEMedH1)

Clinical Nurse Manager (CNMMedH1)

Associate Clinical Nurse Manager (ACNMMedH1)

Senior Medical Registrar (SenRegMedH1)

(Letters in parenthesis are code names from participant transcripts)

In addition to interview and focus group data, documentation pertaining to the care of acutely unwell ward patients was reviewed and is presented as Table 22. 
Table 22. Documents reviewed for Medical ward A

Ward meeting minutes January - June 2009 (monthly meetings)

Medical Unit Nursing Quality Report (January to September inclusive)

Collaborative Care Model Medical Floor

Ward Nursing Rosters 12 weeks August/September/October

Critical Care Outreach Service Annual Report 2009

\subsubsection{Workload}

According to nursing participants, a very heavy workload was a key factor which affected the care of acutely unwell ward patients on this ward. Patients with many co morbidities required lots of care. The nursing participants stated that the ward was 'known' for being very busy and the workload was unpredictable and relentless.

"I think when you come onto a ward like this anything can happen.

Expect the unexpected. And you know usually, you know at any time anything can go wrong" (FGnursex6MedH1)

Nursing participants at times appeared overwhelmed with the volume of work to be completed. 'Having time' was a phrase often used when discussing caring for acutely unwell patients. Nursing participants talked about the difficulties of caring for someone who was acutely unwell while seeing to the more routine needs of other patients they had been allocated.

"It's not because nurses don't want to, they can't. They can't be everywhere at once and if you've got somebody however you want to phrase it, going off or got lots and lots of pain requirements or med requirements, it's very hard to stay there knowing you've got five or six patients on the ward and they're not getting seen to" (FGNursesMedx6H1)

Nursing participants described feelings of guilt in these situations and used terms such as 'sacrifice' when explaining how they cared for one patient to the perceived detriment of another. They wanted patients who were acutely unwell to be moved off the ward to more specialist areas so that they could return to those patients who they believed were being neglected if their attention was focused on one acutely unwell patient. 
Nursing participants talked about prioritising their workload as a means of making time to care for acutely unwell ward patients; however, more senior nursing participants stated that the nurses' priorities were the wrong ones at times.

"we've got a big culture mix of nurses we do have certain cultures of nurses that take on board the fact that they feel, their priorities are washing patients and giving them medications rather than, they're not reactive" (CNEH1)

Furthermore, patient allocation practices were an issue, according to the CNM. The CNM felt that at times staff were given a heavy patient load and rather than question their allocation, nursing staff struggled on. The CNM was working with staff to encourage them to be more careful and flexible in their allocation practices and to challenge any inappropriate allocation.

Nursing participants spoke of being constantly short staffed and this, combined with a poor skill mix, was the main factor which affected the care of acutely unwell ward patients on their ward. When asked what single process or resource would help them care for acutely unwell ward patients, participants believed that more staff were required. More staff included more HCAs and medical staff.

The numbers of staff employed in the ward supported the perception the ward was short staffed. At the time of the study there was a 6.5 FTE vacancy rate. However, in an attempt to increase the nursing numbers the CNM had recruited three new graduate nurses, who were to commence in January 2010. On review of the ward meeting minutes (March 2009) it is noted that the ward was overspent on its staffing budget due to casual and agency nurse use, and therefore some budgetary limitations had been placed on the CNM's ability to meet the current staffing short falls.

Several strategies had been put in place by the CNM and ACNM in an attempt to stay within budget. These included meeting regularly with the coordinators to discuss staffing issues; additional staff being 'signed off' by the CNM; managing staff annual and educational leave to ensure internal cover minimised casual and agency use; and keeping sick leave within budget by using the un-appointed FTE to cover sickness. The average number of agency and casual staff used over the nine 
months prior to the study period indicated that 82 (range $37-138$ ) eight-hour shifts per month were filled using agency and casual staff. This equates to $4.1 \mathrm{FTE}$ based on a 40 hour week, and also indicates that casual or agency staff covered $6 \%$ of all shifts. According to the data collected by the hospital's nursing rostering management system (One Staff), the main reasons for casual and agency use were sickness and vacancies.

Nursing participants also believed that experience in caring for the acutely unwell ward patient was relevant. More experienced nurses were able to make quicker, more accurate assessments of patients. Therefore if there was a lack of experienced nurses on a shift rendering the skill mix as poor this could adversely affect the care of acutely unwell ward patients. Even though the CNM aimed to have at least two senior staff members on all shifts, when there was no ACNM available, senior staff coordinated shifts which reduced the number of senior staff available to care for patients. According to nursing participants this most commonly occurred on afternoon shifts, night shifts and at weekends. Additionally according to the documentation reviewed skill mix was adversely affected by sickness rates and casual and agency staff usage.

\subsubsection{Teamwork}

Despite the busy workload, teamwork between nurses on the ward was perceived as good when caring for acutely unwell ward patients. When a nurse was looking after an acutely unwell patient, the other nurses on the shift would care for the rest of his/her patients. This is noteworthy since the organisation had recently (2008) tried to introduce a more collaborative team approach model of care as evidenced by a new policy document entitled 'Collaborative care model - medical floor'. The collaborative care model organised the nurses into two care teams, where each nurse had specific patients allocated to them who remained their responsibility throughout the shift. The intention was that the team worked together at the beginning of the shift to plan and share workloads and breaks. It was also hoped that this model would allow inexperienced nurses to learn from more senior colleagues by working alongside them. 
Within the document there was a checklist of how a shift might look using this care model. This consisted of a list of tasks and responsibilities that must be completed during a shift with a time line for when these tasks should be completed. The original team nursing model had to be abandoned as it was not sustainable due to the skill mix which, according to the document, was high in junior and inexperienced staff within the ward. A version of team nursing with two members of staff had recently been trialled.

"They are trying to get the focus on this ward that you work together more and more as a team to help with the patient load. One system they tried wasn't quite so successful. They one they're trying at the moment seems to be a little bit more successful which is working pairs rather than in teams. That seems to be a little bit more successful probably because it's a little bit closer knit. But again it comes down to the mix that's on and that's what it depends on really, yes" (NursBMedH1)

Participants described a supportive ward culture during emergency situations. In less acute situations asking for help was seen as difficult at times with nurses 'struggling' on. Explanations for this were that nurses had previously asked for help, been given it but had then been constantly reminded that they had asked for help when they didn't need it or weren't forthcoming in helping others themselves. Alternatively, they had asked for help and no one had helped them; therefore they believed it was futile to ask for help again. There was also the more common perception that everyone else had the same heavy workload so it was pointless asking as no one would have time.

Other teamwork difficulties were due to medical staff's lack of recognition of nursing staff's levels of clinical experience. Nursing participants spoke of their frustration with junior medical staff that were on a short rotation through the ward and consequently were unaware of their experience and expertise. The nurses on the ward perceived that because the doctors did not know them they did not trust or value their opinions in relation to the recognition and management of acutely unwell ward patients. 


\subsubsection{Communication}

Two main factors were highlighted regarding communication on Medical ward A. These were, firstly, communication between nurses when handing over patient information between shifts, and secondly, getting a timely response from medical staff for urgent reviews.

Issues that affected communication between shifts were the time available for handover and the effective use of handover tools. Having time to hand over was challenging particularly between the night and morning shifts. The workload on night shifts was perceived as significant as staffing numbers dropped to three nurses plus one HCA, who was shared across the medical floor. The ward manager felt the night staff were tired which often led to them missing things out in their handovers.

"sometimes even on the Dictaphone handover that we have you don't get the impression that that patient's quite sick just by the way you know especially if it's night to a.m. handover they're quite tired and sometimes they do forget to hand things over"(CNMMedH1)

At the time of the study, the Dictaphone was located in the staff room. Towards the end of the shift each nurse would go into the staff room and dictate the details of their patients' condition and the care delivered during the shift. The oncoming staff were also given a handover sheet completed by the coordinator of the previous shift. The oncoming shift then listened to all of the recordings dictated by the previous shift and wrote down what they considered to be pertinent information on the handover sheet they had been given.

The handover sheet was considered an issue in that it was not always up to date or accurate, which participants believed further compounded the communication factors in relation to the care of acutely unwell ward patients. In addition, a journal entry written during the field work phase of this study notes that handover took place whilst other staff were either talking or clearing up lunch dishes. Furthermore, staff came in and out of the staff room and the phone rang unanswered several times. This made it very difficult to hear what was being said on the tape recording. Finally, participants believed that, due to their busy 
workloads, there was no time for any clarification of patient care issues which may have arisen from the Dictaphone handover.

The second issue regarding communication was getting a response from doctors to requests to see patients. Nursing participants felt that medical staff didn't respond quickly enough to their requests to see patients. The nursing participants described situations where they rang to request an urgent review which they felt was not responded to appropriately.

"Or they'll brush you off and say oh no we'll deal with it shortly or they'll brush you off and say oh no just make a note of it and I'll get around to it when I can. Or you know they'll brush you off and say oh can you get somebody else to have a look at it for you kind of thing. You know so they tend to just brush you off and they won't act upon it straightaway when you want it to be acted upon straightaway and you ask them to do that and they just won' $t$ " (NursBMedH1)

However nursing participants felt that getting a response from medical staff was improving due to the introduction of the hospital-wide computerised communication system (CCS).

Both the nursing and medical participants viewed the communication system as streamlining requests and much more efficient. Nurses described the old system of paging doctors after hours and leaving job lists on 'bits of paper' on the ward as precarious and frustrating. The 'bits of paper' often got lost and the house surgeons spent lots of time answering their pager, which constantly interrupted them and prevented them from working efficiently.

"And that got very, very frustrating on the ward and it also could delay assessments of patients because they're so busy answering the pager or they come to the ward and they look at the bit of paper and they go through reams of paper and they have no idea how to prioritise and which one was written first and which one do I see first" (FGNursesMedx6H1)

However, nursing and medical participants expressed the view that a tool was only as good as those who used it. Staff needed to be judicious in their use of the CCS and call doctors when their patients required urgent review.

"That's where red's come from. I think nurses, especially coordinators are getting more aware of what the nurses are putting 
on there and can actually say oh no we need to ring that"

(FGNursesMedx6H1)

This was because the system has no way of alerting medical staff to what has been listed. Unless doctors were seated in front of a computer terminal they had no way of knowing what jobs had been listed. As a consequence participants believed there could be delays in acutely unwell patients being urgently reviewed.

\subsubsection{Summary}

The domain to emerge from the interview and focus group data for Medical ward A was healthcare workforce. Table 23 presents what the participants believed were the factors most affecting the care of acutely unwell ward patients were on Medical ward A.

Table 23. Domains, themes, and factors Medical ward A

\begin{tabular}{|l|l|}
\hline \multicolumn{2}{|c|}{ Healthcare workforce } \\
\hline Workload & Having time and coping \\
& Allocation \\
& Nurse to patient ratio \\
& Skill mix \\
\hline Teamwork & Support \\
& Clinical experience \\
\hline Communication & Responding \\
& Breakdown in communication \\
\hline
\end{tabular}

\subsection{SURGICAL WARD B}

\subsubsection{Setting the scene}

At the time this study was conducted surgical ward B had 27 beds, which were divided between general surgery (20 beds), ENT (2), and gynaecology patients (5). There were six general surgeons, six gynaecological surgeons and three ear, nose and throat (ENT) surgeons who admitted to the ward. Consequently the ward was 'on take' every day. The ward had a combination of four-bedded bays and single rooms. One of the bays was designated as a 'close observation room' (COR). This bay was situated closest to the nursing office and was used for patients who were the most unwell or required the closest observation. 
On entering the ward there was a large open office area where there was seating for health care staff to read notes, access computers or write patient notes. There was a desk for the ward clerk. There were separate offices for the CNM, the $\mathrm{CNE}$, a seminar room, and a doctor's room. There was a large staff room located by the patient day room which had large windows letting in natural light. It had a table and chairs and two sofas where staff could relax. There were several notice boards which were dedicated to staff training and education which contained notices regarding upcoming study days. These were all in date. There was a large whiteboard which had the details of three upcoming social events. The ward also had a pre-assessment room, a treatment room, and a large preparation room where drugs and other supplies were kept.

\section{Ward Personnel}

The ward was budgeted for 26.9 FTE. Included within this number was one FTE for the ACNM and the pre-assessment nurse. However the budgeted FTE did not account for the CNE or the Health Care Assistants (HCA). There was an additional 4.6 FTE allocated to these personnel (HCA 4.0 FTE, CNE 0.6 FTE). There were currently 40 staff employed with a vacancy of 3.0 FTE. The roster was split into Senior, Middle, and Junior sections. Within these sections the CNM identified 11 members of staff as senior, ten as intermediate, and nine as junior. The criteria used to designate whether a nurse was senior or not was dependent upon their years of experience, their ability to think critically, to problem solve, and demonstrate leadership abilities. The CNM believed that the PDRP was not a reliable measure in that some very senior and capable nurses had elected not to participate in the PDRP framework.

The ward employed two ward clerks whose role was to answer the phones, collate patient notes, book appointments, and meet and greet visitors to the ward. The ward clerks worked shifts which cover from $0730-1600$ and 1400 until 2130. This was from Monday to Friday only. There was no ward clerk cover over the weekends.

The CNM delegated the rostering responsibilities to a senior nurse. The model was self rostering with no explicit rules associated with this. Nurses requested their 
shifts and were generally accommodated. Staff had to do three nights per month regardless of FTE. There was one nurse on permanent night duty but the staff generally rotated through all shifts.

Monday to Thursday the ACNM coordinated the morning shift. The ACNM allocated the patients to the afternoon staff and decided on who would coordinate the afternoon shift. There were no regular coordinators for the afternoon shifts and the choice of coordinator depended on seniority. The afternoon coordinator also took a patient load and was responsible for supervising new graduates and student nurses. The CNM noted that often the afternoon coordinator may also have the highest acuity patients because they were usually the most senior. She also noted that because of the above responsibilities staff had been reluctant to volunteer for this role, hence the necessity to allocate this role. There was no formal training for coordinators.

\section{Patient Acuity Tool}

The ward had its own patient acuity system called the Patient Classification Unit with which staff calculated patient acuity on a shift by shift basis.

\subsection{Surgical ward B: Within-case analysis}

The main domain to emerge from the interview and focus group data from Surgical ward B was healthcare workforce. This included the themes of workload, teamwork, communication, and leadership, roles and accountability. The second domain to emerge was organisation. The only theme to emerge from organisation was organisational systems and processes. Participants who took part in interviews and focus groups from this ward are listed below in Table 24.

Table 24. Participants from Surgical ward B

Surgical Nurses $\times 6$ (FGNursesSurgx6H1)

Surgical Nurses $\times 4$ (FGNursesSurgx4H1)

Clinical Nurse Educator (CNESurgH1)

Clinical Nurse Manager (CNMSurgH1)

Associate Clinical Nurse Manager (ACNMSurgH1)

Senior Surgical Registrar (SenRegSurgH1)

(Letters in parenthesis are code names from participant transcripts) 
In addition to interview and focus group data, documentation pertaining to the care of acutely unwell ward patients was reviewed and is presented as Table 25 .

\section{Table 25. Documents reviewed for Surgical ward B}

Surgical Unit Nursing Quality Report (January to June inclusive)

Coordinating on an AM shift - coordinators job list

Ward Nursing Rosters 16 weeks June/July/August/September

Draft Workforce development plan Surgical Unit

Patient Classification Unit - Instructions for scoring patients

\subsubsection{Workload}

The nursing participants from this ward described a very busy environment not only in respect to the complexity of patients who had multiple co morbidities but also the rapid turnover of patients.

"And I just thought you know they're taking on much higher acuity patients, obese patients and they're saying they're doing them as day cases. They're talking about pushing more day cases through you know from the top, they want all these case weights pushed through otherwise you don't get the funding" (FGNursesSurgx4H1)

Nursing participants felt that they were always working at 'full capacity' and talked of the past when there were periods of time that were not so busy, where they had time to talk to patients and get to know them. In addition nursing participants believed that tasks which were not directly related to patient care such as documentation had increased over time, which then removed the nurses from the bedside and also increased their workload. The workload was perceived as getting 'harder and harder', and was relentless. Nursing participants talked of shifts where they could only complete essential aspects of care for their patients. At other times staying on after their shift had officially ended was the only way to ensure that they completed their care for their patients.

"I mean you've got to go home knowing that you've done all that you possibly can and so to do that sometimes you have to work longer than what you would, longer than the normal eight hour shift but just to go home knowing that you've done everything, you have to work over time to get it done" (FGNursesSurgx6H1)

Their distress when they didn't get everything done for their patients was clear. 
"And you can only do one thing at a time and when things get missed, it just ends up being a disastrous day for myself. And you go home feeling awful "(FGNursesSurgx6H1)

More senior nursing participants discussed the ability to prioritise workload effectively as being dependent upon confidence and experience. The CNE believed that some junior staff didn't like handing over tasks to the next shift as they perceived this to be a reflection of their inability to cope with their workload. There were differing views regarding asking for help. Some nursing participants believed that the ward culture did not support requests for help as staff were expected to cope with their patient load. Other nursing participants described a ward culture that was conducive to asking for help, although nurses were unlikely to offer to help because of their own workloads.

"And so it gets harder and harder to you know. No one will turn down a request to help somebody else of course but you can't really get yourself immersed in anything that's going on with other people's patients because you have to keep up with your own" (ACNMSurgH1)

In some situations the senior nursing participants were able to gauge when staff were struggling with their workload and reported their concerns to either the ACNM or the CNM.

Allocation of workload was also discussed as a factor which could adversely affect the care of acutely unwell ward patients. On this ward, patients were preallocated by whoever was coordinating the preceding shift. This could cause problems at times as patients' conditions could change over the course of the day. As a consequence, the intensity of an allocated workload could alter dramatically. According to nursing participants the ward culture supported staff approaching the coordinator to ask for workloads to be reallocated if they were considered to be inappropriate. Nursing participants felt that this was necessary as the coordinator was unaware of the extent of care required for some patients.

"I've gone to the coordinator and said this person's got too many patients I'll take one or I suggest that someone else takes one. Because we are looking after them on a day to day basis even the coordinator doesn't know how much time people take" (FGNursesSurgx4H1) 
In addition to turnover and complexity of patients, nursing participants believed that there were not enough staff. The ward was 3.0 FTE nurses short in addition to having five nurses on long-term sickness leave. Staff would occasionally have to work double shifts to cover the roster. Despite staff being rostered onto three nights per month irrespective of their FTE, night shifts were particularly difficult to cover. The CNM was actively advertising to meet the staffing shortfall but was having difficulty filling the positions; a situation which she believed was due to a national nursing shortage. When shifts were short staffed the ward tried to fill the gaps with casual pool nurses. However, according to the CNM frequently there were no casual pool nurses available and the CNM was encouraged to ring her own staff to see if they could meet the shortfall. However this was not without its disadvantages.

"So I think there's so many times I have rung the casual pool and said look we haven't got anyone. You have to try your staff, just start there first which is a bit unfortunate really because that just means that your staff are getting used more, getting more tired" (CNMSurgH1)

Sickness and vacancies were the main reasons for casual and agency use on this ward. The quality report for the surgical ward reports sickness as number of shifts per calendar month. In addition to five members of staff on long-term sickness, staff were off sick sporadically. According to the quality reports for the months of January to June the average number of sickness shifts per month was 22 (range $12-31$ ), which demonstrated that $1.5 \%$ of all rostered shifts over 28 days had at least one member of staff off sick. This equated to 1.1 FTE. The average casual and agency staff usage over the same reporting period was 42 shifts (range 36 to 49 ) and equated to 2.1 FTE. This also demonstrated that $3 \%$ of shifts had at least one member of casual or agency staff rostered. The CNM described comparing the surgical ward with the medical floor in relation to staffing.

"Well they've got, I don't know how many health care assistants they have on, they have more than we do. I did a whole analysis of it once when I was trying to argue to get more FTE for healthcare assistants. They've got a patient coordinator on the p.m. that doesn't take patient load and just that would be great and they've 
got discharge planner and you know all these other people up there" (CNMSurgH1)

The CNM was unsure why these staffing disparities existed.

\subsubsection{Teamwork}

Within the theme of teamwork, clinical experience and relationships were important factors affecting care of acutely unwell ward patients. There were a core of senior nursing staff who had been employed on the ward for several years. The ACNM described how many of the senior nurses had come to the ward as junior nurses, worked there for a couple of years, gone overseas to work and then had come back and stayed. She described the relationships that had developed over the years.
"so there's a group of about if I counted them off I could get to ten or twelve or fifteen nurses who've been through a whole sort of a life cycle together. You know we've watched our children grow up and we've shared a lot of experiences, life experiences. We've had the divorces and the remarriages, we've had the whole bit and everyone's gone through it together. And, there's just years of experience there and they've been fantastic mentors to a lot of junior staff" (ACNMSurgH1)

Both the CNM and the ACNM thought that it was extremely important that staff developed social relationships as they believed this enhanced team working. This included socialising with the medical staff who worked on the ward. This was supported by the number of ward social events being advertised on the white board in the staff room. Furthermore the CNM described an incident when a junior nurse who was a fairly new member of staff had not recognised when a patient was becoming acutely unwell, had not been able to cope with her workload but had not asked for help.
"And then something else, I know this is very touchy feely but she doesn't, she hasn't begun to socialise with us as a group and so I'm wondering whether she needs to do that you know to develop some relationships, I think that's really important when you're working as a team at work" (CNMSurgH1)

According to the CNM, socialising together enhanced good team relationships, staff became 'close' and therefore were more willing to help each other out. The 
nursing participants also described knowing the registrars very well and were therefore not afraid to bypass the house surgeon and contact them directly should the situation warrant it.

Nursing participants believed that the ward was fortunate to have a core of senior nurses who could pass on their skills and expertise to more junior staff. The ACNM described some of the senior nurses as 'old fashioned nurses' who delivered 'thorough' nursing care, had 'kept up with the times' but were not interested in undertaking post graduate education.

"And I truly believe that that's one of the things that's held this particular ward in good stead over the years" (ACNMSurgH1)

The ACNM believed these nurses had good assessment skills and were able to detect deteriorating patients quickly as they 'knew what to look for'. In contrast, junior nurses were much more task focused, formulating rigid timelines for the completion of their workload.

"I've noticed they all come out these days and they write their plans, their timelines on the back of their patient information sheet. You know 9 o'clock, 10 o'clock, and this is what I'm going to do. Well that is great to have that as a guide but you quickly learn that actually nursing is not a predictable job. Patients are unpredictable and the patients we get are unpredictable" (CNESUrgH1)

Some senior nursing participants felt that junior nurses were less likely to be able to adapt their timelines to meet the needs of an acutely unwell patient, which was factor that could impact on care of this patient group.

Also associated with clinical experience was skill mix per shift. Nursing participants perceived that morning shifts often had more senior staff rostered on. According to the CNM the desired minimum number of seniors on night duty and afternoon shifts was two per shift. On review of the rosters there were 11 nurses classified as senior staff on the ward, which would facilitate the desired number of seniors required per shift. However sickness and annual leave often impacted on these numbers, meaning that according to the ward rosters the most senior person on a shift was from the 'middle' section of the roster. 


\subsubsection{Communication}

Communication factors were associated with the length of time junior doctors took to respond to calls for an urgent review. The CNM believed that the key factor which affected the care of acutely unwell ward patients was when it had been 'difficult to get hold of a doctor'. Nursing participants described situations where they had phoned the house surgeons and given a detailed handover using the SBAR tool but still their request for an urgent review had not been facilitated.

"You are trying to be really clear and concise about what you're trying to say, you know and they still don't get it. And won't come. And it's like you're in a situation where you need help and your resources have just expired and where else do you go and it's the patient that suffers" (FGNursesSurgx4H1)

Senior nursing participants were confident enough to bypass the house surgeons and go straight to the registrars, although these participants felt that this may not be the case for more junior nurses. Nursing participants believed that on many occasions junior doctors were busy elsewhere, which is why they did not respond quickly. A further difficulty with getting doctors to respond was when the patient was an outlier on the ward and therefore the nursing staff were trying to get hold of a different specialty to surgery.

The doctors' level of experience also affected team working. The senior nursing participants regarded themselves as more experienced than the new house surgeons and some felt that part of their role as senior nurses was to guide and nurture the junior doctors.

"I've just become more acutely aware of that in recent years that with probably my own maturity or whatever you just start to realise that part of our role is nursing some of these young doctors through these particular sorts of cases" (ACNMSurgH1)

If this knowledge and experience was not acknowledged by the junior medical staff, that could lead to communication difficulties.

\subsubsection{Leadership, roles and accountability}

The inability of the coordinator to provide shift leadership was the key factor perceived by participants to affect the care of the acutely unwell ward patient 
within this theme. The CNM had overall managerial responsibility for the ward and apart from Fridays, when she coordinated the morning shift, the CNM did not work on the floor. The ACNM worked Monday to Thursday and coordinated the morning shifts but did not take a patient load. On all other shifts the coordinator took a full patient load. This was an issue, according to nursing participants.

"I'll tell you the other thing that comes out of that is, I had that lady plus my other four patients and I was coordinating for three days. I was absolutely exhausted by the end of that. It was too much, so one of the difficulties here on this ward is that you have to coordinate as well as take a full workload" (FGNursesSurgx4H1)

The role of the coordinator was perceived as being very stressful because of the very heavy workload. Coordinators were senior nurses; therefore they often allocated themselves the highest acuity patients. In addition their role involved managing the ward in terms of admissions and discharges, entering acuity data, supervising junior staff, giving intravenous drugs for enrolled nurses and 'keeping an eye' on casual and agency staff.

There was no formal training programme for coordinators. However, there was a 'job sheet' which outlined what was expected of coordinators. The job sheet consisted of a list of 20 tasks that the coordinator was expected to complete before the end of the shift. Some examples of the tasks include attending the doctors' handover, accompanying the doctors on wards rounds and communicating ward round information to the appropriate nurse, ordering and collecting drugs, entering acuity data and updating and printing off handover sheets.

Both nursing and ward managerial participants stated that with so many responsibilities it was extremely difficult to have an overview of what was happening on the ward and therefore this could adversely affect the care that acutely unwell ward patients received. In addition, recently staff had been 'arguing and refusing to coordinate', leading to the ACNM having to allocate who was going to coordinate as no one would volunteer. When asked which resource or process she believed would most support the care of acutely unwell ward patients, the CNM stated that a coordinator that didn't take a patient load would be what she would request. 


\subsubsection{Organisational systems and processes of care}

Within this theme the main factors described by participants as affecting the care of acutely unwell ward patients were service initiatives and organisational policies. Service initiatives which had supported participants in their care of acutely unwell ward patents were CCOS and EWS. Participants described the support and reassurance that CCOS provided.

Nurs1 "It's been a lot better because we can call them straightaway.....

Nurs2 "And they've got the time to sit and read the notes and get the whole story

Nurs3 "And they, and they can get people to do things quicker" (FGNursesSurgx4H1)

Nursing participants viewed the CCOS as being able to get a quicker response from the medical staff which saved the ward nurse's time. Nursing participants believed that the CCOS were more experienced in the care of acutely unwell patients and had more authority with regard to referring patients for review.

Having an early warning score was also viewed as a welcome service initiative in the care of acutely unwell ward patients. Most participants considered it as a supportive tool which assisted nurses in getting attention from medical staff by providing a way of packaging deterioration. Managerial nursing participants described the tool as an adjunct to care and as a 'safety net'. Recording EWS was part of the ward's KPIs. The ward's quality report demonstrated that EWS uptake was variable. The average compliance over seven months was $80 \%$. The CNM believed that, initially, some staff had been sceptical regarding the efficacy of the score, but over time there was more of an acceptance of the value of the score.

An organisational process which both nursing and medical participants viewed as detrimental to the care of acutely unwell ward patients was the care and management of outliers. Nursing participants described the difficulties associated with caring for these patients.

"And I just think sometimes when you've got outliers in the surgical ward you're best to really not give us the most complex ones 
Complex syndromes that we've never ever heard of or dealt with or you know" (FGNursesSurgx4H1)

Nursing participants felt that they had limited knowledge regarding caring for these patients. To compound matters they also experienced difficulties in getting hold of the doctors caring for these patients. When the doctors did review their patients they wrote in the patients' notes, but did not verbally hand over their requests regarding patient care, leading to delays in patients receiving the prescribed care.

\subsubsection{Summary}

The domains to emerge from the interview and focus group data for Surgical ward B were healthcare workforce and organisation. Table 26 presents what the participants believed were the factors most affecting the care of acutely unwell ward patients on Surgical ward B.

Table 26. Domains, themes, and factors Surgical ward B

\begin{tabular}{|l|l|}
\hline \multicolumn{2}{|c|}{ Healthcare workforce } \\
\hline & Having time and coping \\
& Task focus \\
& Allocation \\
& Nurse to patient ratio \\
& Skill mix \\
\hline Teamwork & Relationships \\
& Support \\
& Clinical experience \\
\hline Communication & Responding \\
& Breakdown in communication \\
\hline Leadership, accountability and roles & Leadership roles \\
\hline \multicolumn{2}{|c|}{ Organisation } \\
\hline Organisational systems and processes \\
of care & $\begin{array}{l}\text { Service initiatives } \\
\text { Organisational policies }\end{array}$ \\
\hline
\end{tabular}

\subsection{HOSPITAL 2}

Hospital 2 was located in the North Island of New Zealand. At the time of the study it had between 600 and 800 beds and served a population of between 300,000 and 500,000 people. It was a tertiary referral centre with teaching hospital status. The hospital employed between 2,000 and 3,000 staff and treated between 40,000 , and 60,000 in patients annually. Many specialist services were available, 
including cardiac surgery, adult and neonatal intensive care, neurology and neurosurgery, oral and maxillofacial surgery, paediatric surgery and plastic and vascular surgery.

\subsection{Hospital 2: Within-hospital analysis}

In order to provide contextual detail for Hospital 2 senior and executive management, medical staff, two After Hours Support Nurses (AHSN), and a senior nurse who was acting as a project manager were interviewed. The titles and specific numbers of these participants are illustrated in Table 27.

\section{Table 27. Organisational participants Hospital 2}

Chief Medical Advisor (EMTH2)

Chief Operating Officer (EMTH2)

Director of Nursing (EMTH2)

After Hours Support Nurse x 2 (AHSNurs1H2),(AHSNurs2H2)

Senior Manager Medicine (SenManMedH2)

Senior Manager Surgery (SenManSurgH2)

Senior Nurse (SenNursH2)

(Letters in parenthesis are code names from participant transcripts)

These participants were asked to discuss what they believed the factors were which affected care of acutely unwell ward patients from an organisational perspective. In addition to interview data, documentation pertaining to the care of acutely unwell ward patients was reviewed. Table 28 lists the documents reviewed as part of the organisational analysis for Hospital 2.

\section{Table 28. Documents reviewed for Hospital 2}

Patient Safety Pamphlet for staff

Nursing and Midwifery project document

Early warning score project update

Health Care Assistants - Direction and delegation of tasks - Hospital memorandum from DoN to all staff

Email from Group Manager to all staff regarding budgetary restraints and staffing levels

Critical Nursing Sick leave levels - Hospital Memorandum to all staff from Group Manager

All three domains of healthcare workforce, organisation, and education emerged from Hospital 2. Of the three domains to emerge, the main domain was organisation. The main themes to emerge from organisation were culture and 
environment and organisational systems and processes of care. The main themes from organisation will be discussed first, followed by the key themes from healthcare workforce (workload, teamwork and communication) and education (skills and knowledge deficit).

\subsubsection{Culture and environment, and organisational systems and processes of} care

Within Hospital 2 the themes of culture and environment and organisational systems and processes of care were closely linked and are therefore discussed together. The organisation was striving for a culture of 'no avoidable deaths and no avoidable harm'. The organisation had identified a number of incidents and serious adverse events (SAE) which had

'identified examples of unsatisfactory in-patient monitoring practices, delayed recognition in patient condition leading to delays in treatment, and situations of poor communication between health professionals'. (Taken from staff 'patient safety pamphlet')

According to some executive management participants the hospital relied on outdated organisational processes and policies which did not work.

"So to go back to your question we haven't got good systems in place. We rely on the nurse's ability to escalate processes that have been in place forever" (EMTH2)

Furthermore, there was a perception that the organisation relied on 'personalities and perseverance' rather than robust systems and policies to address patient safety. As a result the hospital was in the process of introducing several new patient safety service initiatives at the time the study was conducted. The hospital had employed a senior nurse to project manage the development of an early warning score and a medical emergency team (MET). The hospital had also rolled out SBAR as a communication framework for healthcare staff and was formulating a colour coded observation chart which would alert nurses to patient observations which were outside normal parameters. Several wards were also implementing 'The Productive Ward; Releasing Time to Care' programme, which is based upon a series of 11 modules which focus on improving ward processes and environments to help nurses and therapists spend more time on patient care, 
thereby improving safety and efficiency. Finally, quality coordinator positions had been established for each service to help develop and implement the work required to meet the organisation's patient safety goals.

In addition to all of the changes associated with the patient safety agenda, the organisation was also in the throes of a nursing and managerial restructure. The purpose of the restructure was to clarify nursing and midwifery leadership accountabilities. A restructure document had been formulated and proposed a new nurse management structure across the hospital focussing on clinical leadership, with the aim of putting the hospital management into the hands of clinicians. At the time this study was conducted the restructure change proposal document was out for consultation.

One EMT participant believed that accountabilities and responsibilities associated with leadership positions needed to be clarified and strengthened. The organisation had held discussions with staff and these had demonstrated that currently there was confusion within the organisation regarding levels of authority within leadership positions and that this led to organisational dysfunction. This EMT participant believed this confusion had led to frustration amongst staff and ultimately had affected patient care.

The number and pace of the changes associated with both patient safety and the nursing structure was of concern to some nursing and management participants. For some, making too many changes too quickly meant that the changes were not thought through sufficiently nor implemented properly. For others, the implementation of the patient safety agenda had taken a back seat until the results of the restructure were announced, as they were unsure whether they would still be employed and did not want to start something they might not finish.

\subsubsection{Workload}

Participants' views regarding nursing workload differed, depending upon their position within the organisation. Those staff who worked on the wards or had direct contact with patients and ward staff believed that the nurses' workload was very heavy and was an important factor affecting how nursing and medical staff cared for acutely unwell ward patients. 
"But yes most of their [surgical ward] nights are very busy. Their workload doesn't halve by any means. I mean they're not having patients, well they do have patients that come and go from theatre but not as often as on the morning shift. But yeah, on the [medical ward] as well. They can have some very busy very sick patients. It's all to do with the acuity of the patients they're not better at night than they are in the day" (AHSNurs1H2)

These nursing participants believed that the situation was further compounded by the wards being short staffed and having an inadequate skill mix. However, some executive management participants believed that patient acuity had not necessarily increased and that the hospital was adequately staffed, with sufficient numbers of senior staff to provide a good skill mix.

"We've got 83\% of our staff are senior that says they're on step four or five of the nurses MECA which means they've been working for at least four or five years. Agreed that doesn't mean, I absolutely acknowledge that doesn't mean it correlates to seniority or ability or vigilance. So I don't think we can actually blame that any more" (EMTH2)

Again views differed regarding the number of senior staff in the organisation, depending on the participant and which data source the participant was using to support their views. The EMT used pay roll data as their way of estimating the number of senior staff. Other senior managers used rostering information to provide their data. These senior managers believed that at least $33 \%$ of staff should be senior in the wards, but that was not the case. Furthermore, if the numbers of senior staff were unsatisfactory, the absence of just one senior nurse could have a serious impact on care delivered on the ward.

"And all you need is a sick leave or a bereavement leave or something like that to take out a senior nurse and then you get intermediate junior nurses who have even less skill and less ability to manage or to think more laterally around what it is I need to be doing" (SenManSurgH2)

However, members of the EMT believed that nurses were so focused on how busy they were that this prevented them from looking at alternative ways of working.

"I think we need to stop using busyness or almost relying on the tyranny of busyness as an excuse. This is our job. We haven't developed or hung onto the ability to delegate appropriately so the 
$R N$ quite often takes on all of the roles that they could delegate to healthcare assistants, to ward clerks, gosh to other members of the team, nursing team I mean. It's about good allocation of processes at handover. Not just doing the historical we've all got four or five patients. Some people could take six patients alone, that one to take three or four because they're really sick you know. It's about colocating patients that are unwell. It's about streamlining your work processes" (EMTH2).

One member of the EMT also believed that rostering practices were 'historically embedded'. Currently, shifts were staffed with the most nurses on a morning shift, with decreasing numbers in the afternoon and night. According to this participant this was outdated, given that she believed the hospital's busiest time was from 1400 until 2030. This EMT participant believed that shifting the resources to where they were needed the most would make a difference. However, some senior management and nursing participants questioned whether the realities of the ward environment were completely understood by the executive management.

"I think there is an understanding of how sick the patient may be but I don't think they understand what working on the floor is like or the level of knowledge of nurses on the floor at this point in time" (SenManMedH2)

There was also the perception from these participants that dealing with the issue of workload and inadequate staffing was either ignored or perceived as too hard to deal with.

\subsubsection{Teamwork}

Within Hospital 2 relationships were critical to effective teamwork and featured as an important factor that participants believed affected the care of acutely unwell ward patients. One EMT participant maintained that any barriers regarding relationships to the care of acutely unwell ward patients were not at organisational level.

"I would hope nothing from an organisational perspective, I think what may stand in the way is the hierarchies within wards and units really" (EMTH2) 
Some nursing and executive management participants believed the relationship between the junior doctors and nursing staff was the crucible in relation to the care of acutely unwell ward patients. The difficulties in relationships which arose between doctors and nurses were attributed to poor attitudes of individuals or difficult personalities. This affected communication within the team. Participants also believed that relationship difficulties occurred between doctors as a group and nurses as a group. According to some executive management participants, fear was an issue which might prevent junior doctors from asking for help.

Nursing participants described avoiding some individuals because of the attitude shown towards them. Despite having an escalation policy which advised junior doctors on how to get help, there was a perceived reluctance to use it. This was viewed as a factor which could lead to delays in acutely unwell ward patients being reviewed. Some of these unhealthy behaviours were believed to be caused by frustration at some of the organisational systems and processes. Executive management participants appeared to be aware of this and were trying to effect change.

"But what we want is a commitment I think, I'm pretty sure we need a commitment from the chief medical advisor down that poor behaviour of consultants and of registrars and of house surgeons will all be addressed. Because sometimes that poor behaviour is the result of dreadful frustrations that we could sort out. I will undertake to address poor behaviour in the same way for nursing" (EMTH2)

At times poor relationships affected communication between specialties and therefore affected access to specialty care according to some senior management participants. This was particularly relevant to the ICU environment, which was perceived as an 'ivory tower' with very strict gatekeepers.

"I don't think anything will change until we can start getting consultants on board especially in ICU and HDU to realise that it's all well and good them having their nice little ivory tower but it does mean that out on the wards you've got patients who you may not deem them ICU appropriate but they're not getting the level of care they require on the ward because you've got nurses who don't have that multi system level of knowledge"(SenManMedH2) 
These participants believed that delays in getting specialty services to review acutely unwell ward patients adversely affected their care. One way of accessing support quickly after hours was by using the After Hours Support Nurses (AHSN).

The AHSNs were an existing service whose role was to provide support to the ward nurses and junior doctors after hours. These nurses worked across the organisation helping ward nurses care for acutely unwell ward patients as well as providing specific skills such as cannulation. However the after hours support nurses believed that they were underutilised and were concerned that their reporting structure was flawed.

The AHSN's managerial reporting line was to the manager of the casual pool and agency. The AHSNs felt frustrated by this reporting line as they felt it downplayed the value of their role.

"Yes and we know we do a good job, we can, we can do a good job because we go to a problem and if nothing else we're just another pair of hands but we're usually another pair of hands who know quite a bit more and know how to respond in a situation you know so I mean we do a very valuable job but we just feel we've been undervalued" (AHSNurs1H2)

Furthermore, when they saw factors which they believed affected the care of acutely unwell patients they felt they had no one to report their concerns to that had sufficient influence to effect change

"We see it, we report it, we write incident forms, we go to see the charge nurse, we see educators, we like are banging our heads against a brick wall. If we reported to a director of nursing we all feel we'd have more clout and we could get these things changed"(AHSNurs1H2)

The AHSNs felt that ward managers needed to remind the nursing staff that they were there to support them after hours. According to the AHSN participants, on wards where the CNMs had reinforced the use of the AHSNs the nursing staff felt more supported. Conversely, where there was no direction from the CNMs to use the AHSNs, the AHSNs often came across acutely unwell patients by chance and often at a very late stage. 


\subsubsection{Communication}

Within the theme of communication, breakdown in communication and responding were described as factors which adversely impacted on care of acutely unwell ward patients. The SBAR communication framework had been implemented, but there had been no evaluation of its effectiveness at the time of the study. Doctors were supposed to hand over to each other at the end of each shift, but the level of detail in this handover was variable. There was no formal electronic or handwritten documentation of this handover, although some participants felt that this was required.

"It's got to be formally communicated and that's maybe both people sitting down in front of a computer or sitting face to face and saying this is what's going on and here are the patients I'm concerned about" (EMTH2)

This was perceived as being necessary because of the shift and on call systems, where doctors could be asked to review patients they had not previously encountered.

Communication between nursing and medical staff was difficult at times due to a perceived lack of information given when a request for an urgent review was made. Furthermore, some senior and executive management participants believed that nurses were guilty of making requests that were not urgent, sound as though they were urgent. This led to the possibility that junior doctors might ignore genuine requests for urgent reviews.

There was also the perception that there was a block in communication between middle management and ward management.

"Who's stopping you do these things. And it's been quite interesting because I can see why people get frustrated. You find that there's that middle layer of manager that sort of you know knowledge is power. That's often where the blockers are we haven't got the money so you can't have it" (EMTH2)

Conversely, some senior management participants felt that they were powerless to do anything without executive team sign off. This was particularly relevant to recruiting staff. The process for recruitment within Hospital 2 stated that the COO 
had to sign off all requests for new staff, even replacement staff. This meant that it often took three months to replace staff.

"You leave and you're a full timer and I'm a part timer and I want a position and someone else is part time and makes it full time. Now I have to put your name on the piece of paper, I then have to get [Operations manager] to sign it, it goes to her desk and [Group manager] has to sign it and then the COO has to sign it and the general manager to HR has to sign it and then god knows where it goes after that" (SenManSurgH2)

Given that the wards were often short staffed, senior management participants stated this process was lengthy and exacerbated an already difficult staffing situation.

\subsubsection{Skills and knowledge deficit}

Within the theme of skills and knowledge deficit the main factors discussed by executive management participants at Hospital 2 were workforce preparation and assessment skills. Executive management participants felt that undergraduate nurses and doctors were not adequately prepared for the environment they were entering. This was mainly due to having less clinical exposure during their training.

"And so previously nurses were in the wards and involved in clinical care from a really early stage in their training. It happens far less now and I think that's all part of the same picture. That there's a lot more focus on the academic and a lot less on the actual hands on experience and I think both young doctors and young nurses when they do their intern year are on a very steep learning curve" (EMTH2)

The lack of clinical exposure impacted on their ability to care for acutely unwell ward patients because new graduate nurses and junior doctors had minimal experience of looking after this patient group and often were not confident in terms of their assessment skills. Senior management participants believed that nursing staff did not see the significance or importance of assessment skills.

"Like even the respiratory ward if you ask them why they need to do respiratory rate most of them don't know actually or its significance. You know somebody starts breathing heavier and faster or slower what might that mean. They just don't have that level of thinking" (SenManMedH2) 
Other executive and senior participants believed that on wards where role modeling of good assessment practices was not prevalent, junior staff had good assessment practices 'socialised' out of them.

\subsubsection{Summary}

All three domains emerged from the interview and focus group data for Hospital 2. Table 29 presents what the participants believed were the factors most affecting care of acutely unwell ward patients in Hospital 2.

Table 29. Domains, themes, and factors Hospital 2

\begin{tabular}{|l|l|}
\hline \multicolumn{2}{|c|}{ Organisation } \\
\hline Culture and environment & Organisational culture \\
\hline $\begin{array}{l}\text { Organisational systems and processes of } \\
\text { care }\end{array}$ & $\begin{array}{l}\text { Service initiatives } \\
\text { Organisational policies }\end{array}$ \\
\hline \multicolumn{2}{|c|}{ Healthcare workforce } \\
\hline Workload & $\begin{array}{l}\text { Having time and coping } \\
\text { Nurse to patient ratio } \\
\text { Skill mix }\end{array}$ \\
\hline Teamwork & $\begin{array}{l}\text { Relationships } \\
\text { Support }\end{array}$ \\
\hline Communication & $\begin{array}{l}\text { Responding } \\
\text { Breakdown in communication }\end{array}$ \\
\hline \multicolumn{2}{|c|}{ Education } \\
\hline Skills and knowledge deficit & $\begin{array}{l}\text { Workforce preparation } \\
\text { Assessment skills }\end{array}$ \\
\hline
\end{tabular}

\subsection{MEDICAL WARD C}

\subsubsection{Setting the scene}

At the time this study was conducted Medical ward C was a 27-bedded ward consisting of a combination of single rooms and four-bedded bays. There was a medications dispensary, treatment room and sluice and several storage cupboards for dressings, supplies, and equipment. Office space consisted of a doctor's office where the patient notes were kept in two trolleys and a nursing office where nurses wrote their patient progress notes and where handover took place. There was a small separate office for the CNM and the CNE. There was a small staff room in the middle of the ward. This had a table and chairs and some notice boards for education and practice development. 
There were six different medical teams comprising five specialties who admitted to the ward. These were cardiology, renal, endocrinology, general medical, and gastroenterology. Teams had been allocated a colour so that notes could be identified more easily. The ward had recently introduced team nursing. The CNM had organised the teams but each day the nominated team leader allocated within the team, or the nurses within the team self-allocated their individual patient load. The introduction of team nursing had also led to a change in the way handover was given. Nursing staff gave a short sharp global handover in the nursing office and then walked round their patients and gave a bedside handover.

\section{Ward Personnel}

At the time of the study there were 26.5 FTE nursing staff and 5.6 FTE Health care assistants (HCAs) on the ward, totalling 36 members of staff. This demonstrates that most staff were full time. There were no members of staff on long-term sick leave. There were 1.3 FTE vacancies. Turnover of staff had been high in the last 18 months, with six full time staff leaving to go overseas to work. Staff were continually encouraged to take their annual leave and to make annual leave requests at least two months in advance. This was a regular agenda item on the ward meeting minutes. There were a number of overseas trained nurses employed on this ward who tended to book long periods of holiday to return home, although only two members of staff were allowed on annual leave at any one time. The ward had a ward clerk who worked Monday to Friday $0900-1730$ and 1000 1300 on Saturday and Sunday. There was a patient communication device to alert staff to phone calls and queries.

The CNM had recently resigned and the CNE had taken on the CNM role temporarily, as well as continuing with the education role. The CNM position had been advertised and interviews were pending at the time of the study. The coordinator on all shifts took a patient load. The role of the CNM was as an overall manager and, unless circumstances dictated it, did not coordinate on the morning shift. The most senior person on the shift coordinated. There was no formal training for coordinators. 
The roster was divided into three domains of senior, intermediate and junior. Where a nurse was positioned on the roster depended on the number of years employed on the ward. Rostering was done by the CNM. Staff were allowed two requests per month.

\section{Patient Acuity Tool}

There was no patient acuity tool for this ward.

\subsection{Medical ward C: Within-case analysis}

The main domain to emerge from the data for this ward was healthcare workforce to include all themes: workload; teamwork; communication; and leadership, roles and accountability. Within this ward workload and teamwork were very closely linked, so these two themes are discussed together. Ward management, nursing and medical staff were interviewed or took part in focus groups. The titles and specific numbers of participants are illustrated in Table 30.

\section{Table 30. Participants from Medical ward C}

Medical Nurses x4 (FGNursesMedx4H2)

Medical Nurses x2 (FGNursesMedx2H2)

Clinical Nurse Manager (CNMMedH2)

Senior Medical Registrar (SenRegMedH1)

House Surgeon Medical (HSMedH2)

(Letters in parenthesis are code names from participant transcripts)

In addition to interview and focus group data, documentation pertaining to care of acutely unwell ward patients was reviewed and is presented as Table 31.

\section{Table 31. Documents reviewed for Medical ward C}

Ward meeting minutes March, July, August, September, November 2009 Ward nursing Rosters 8 weeks November/December 2009

\subsubsection{Workload and teamwork}

Nursing participants described their workload as 'heavy', due to the number and complexity of the patients they were expected to care for on a shift. This was often compounded by being short staffed.

"So they're looking at picking up six patients [when] you would normally give them, they would normally get between four and five. 
So they, $30 \%$ of the time they'd be looking at picking up a heavy load." (CNMMedH2)

Typically the ward aimed to have six nurses and two HCAs on duty for a morning and afternoon shift and three nurses for a night shift. On review of eight weeks nursing rosters, the ward did not achieve these numbers $46 \%$ of the time.

Furthermore, shifts were down by more than one member of staff (either nurse or HCA) $25 \%$ of the time. This supports the CNM estimation that the ward was short staffed $30 \%$ of the time.

There was an acceptance by participants on this ward, however, that nurses were a scarce resource. Nursing participants stated that if the ward had only four nurses (and two HCAs) on a shift they would be supplied with a casual nurse. However, if the ward had their full quota of nurses and HCAs on a shift but they were busy because of increased patient acuity, they were unlikely to get any additional help.

The ward did not use a patient acuity measure to determine their nursing workload. There had been an acuity measure in place up until 2008, but the ward had stopped using it. The CNM believed that it was no longer used because, firstly the tool was not perceived as accurately capturing the patients' level of acuity. Secondly he believed the 'powers that be' didn't feel that it was being closely monitored enough and that even if the acuity tool demonstrated that the acuity within the ward was high there were insufficient nursing resources to meet the acuity: therefore having the tool was a waste of time.

Nursing participants stated that patients were 'heavy' not necessarily because they were acutely unwell but because they had multiple co morbidities, which meant that they required a lot of care. When a patient within their allocated load became acutely unwell this impacted on their ability to meet the needs of their other patients.

"I think it is about their workload. Sometimes you know as a patient becomes unwell, they've got other patients who are even worse, so they prioritise and dedicate their time or have to ask another nurse to give them a hand" (FGNursesMedx4H2) 
The CNM believed that some of the nursing staff tried to get everything done within an eight-hour period and had difficulties prioritising what was important in terms of care. As a result the needs of acutely unwell patients were not always met. The CNM described scenarios where staff had been encouraged to determine what the main priorities of their workload were.

"But a nurse, they'll always try to get as much as they can get done. Now we're not saying be slack and don't worry about getting all your cares done but when it's acutely busy and they're really flat out [NAME REMOVED] and I have both said look just focus on your acute cares, prioritise and delegate" (CNMMedH2)

Another impact of the heavy workload was that staff became task focused. As staff became more task focused, they were more likely to miss more subtle signs of deterioration, according to the CNM.

"And often they're so sort of focused on trying to get their jobs done and their tasks done that they tend to sort of think more about tasks and they're more focused on getting that done and that can definitely affect here because you know I've come along and I'll be worried about a patient and I'll go and investigate that patient and I'll pick up things that haven't been caught up with" (CNMMedH2)

In an effort to provide support for nursing staff and ameliorate some of the aforementioned issues, the ward had introduced team nursing. The ward was divided into a red and green end.

"We pretty much split the ward into two and there's a team down this end and a team down this end. So when you do find yourself in a difficult spot you can firstly approach your team whereas if they're all individuals it's kind of like well who do I go to" (CNMMedH2)

When the shift was fully staffed, each end had a team leader, two other nurses and an HCA. By introducing team nursing it was hoped that staff would feel less isolated and more supported. The CNM was also trying to foster a good team spirit in relation to patient care.

"We try to actually build the fact that we are a team, we're a 24 hour team. We're there to actually help each other out and there are going to be many days there where you aren't going to get all your work done and there are going to be days there where it's so damned busy that you can actually pass some of it on"(CNMMedH2) 
The CNM was aware of the difficulties regarding workload. When it appeared that nurses were struggling with their load, they were 'shoulder tapped' and given support and guidance about managing their patients. Time was spent reassuring nurses. The CNM believed that, when orienting new nurses to the ward, it was important to promote guidelines regarding acceptable practice on the ward. However, realistically, he felt that staff learned some behaviour from other nurses they were working with which was 'historical' to the ward and not necessarily the culture they were trying to foster.

Nursing participants described supporting each other on the ward where they could, although they realised that this support was given depending upon how busy other nurses were within the ward.

"Sometimes if the other nurses aren't busy then you can call them to help. But on the general medical ward you do have a lot of heavy patients that you know, quite heavy with full cares. You know they need virtually everything done for them so you know a lot of the other staff have patients like that and they are limited to what they can do for you." (FGNursesMedx2H2)

Nursing participants believed that this left some junior nurses without support, which may adversely affect the care of acutely unwell ward patients.

\subsubsection{Communication}

Communication issues described by nursing participants were difficulties in getting hold of doctors and delays in the time between when they called the doctor and when the doctor arrived on the ward.

"You know they keep saying they'll come but they don't show upyeah so that happens sometimes" (FGNursesMedx4H2)

However, some nursing participants felt that this was dependent upon the information that the doctor was given by the nurse requesting a review. If the nurse provided a convincing case they felt that doctors would come straight away. This was a supported by medical participants who described the variability of information in terms of detail that they were given, which affected their ability to prioritise calls. 
This was especially relevant after hours, when medical staff numbers were reduced and they had large numbers of patients to care for, many of whom they did not know.

"And normally when we get a call I personally would like more history as to what's going wrong with the patient rather than the patient's in pain or the patient's etc and I would like a set of obs. And most of the time you get it but it depends sort of on how quickly you get it" (HSMedH2)

Furthermore, the medical participants felt that they were often not on the ward because of the number of patient outliers they were looking after, which also affected their workload and time management.

The CNM was aware of the difficulties regarding communication between nursing and medical staff, and therefore there had recently been a focus on improving communication skills and using the SBAR framework on the ward. Overall, nursing participants believed that once you had the attention of the medical staff, team working between medical and nursing staff was generally good; albeit dependent upon the experience of both the nursing and medical staff.

Nursing participants expressed the view that experienced nurses were more skilful in assessing patients in order to pick up signs of deterioration and their communication skills were more refined therefore they could get the attention of the medical staff more effectively.

"The junior nurses sometimes they do get fobbed off by the house surgeons. You know it will take a senior nurse to go in there and just sort of you know change focus and maybe sort of ask for something in a different manner" (CNMMedH2)

Nursing participants expressed concern regarding the number of junior nursing staff employed on the ward. This was especially relevant when there was a large concentration of junior staff on any particular shift. At the time of the study the nursing roster showed that the largest proportion of staff were classified as junior (14 nurses and 3 new graduates). On examination of the rosters, there was at least one senior nurse on all morning and afternoon shifts. However at least one of the senior nurses coordinated the shift. On night duty there were some shifts where the most senior person on the shift was from the intermediate domain of 
the roster. Given the numbers of junior staff on the ward and the fact that the senior nurses have to coordinate the shift, it is not surprising that the majority of nurses on a shift are described by staff as junior.

\subsubsection{Leadership, roles and accountability}

The multiple responsibilities of the coordinator were felt to affect care of acutely unwell ward patients on this ward. Participants spoke of the difficulties associated with taking a patient load and coordinating a shift.

"[You] should take less workload but it hardly happens you have to take a full workload and you have to help a new grad nurse and student nurse and you've got a sick patient and other people ask for a hand it's pretty hard you know" (FGNursesMedx4H2)

The nursing participants stated that because the coordinators' workload was so heavy, they were unlikely to be able to help other members of staff despite being the most senior person on the shift, unless there was an emergency. Participants described the stress of being a coordinator.

"You know they're often inundated and they've got their patient load as well so they get to a point where they get quite strung out. And it gets incredibly tough for them" (CNMMedH2)

Nursing participants described raising the issue of the coordinators' workload at ward meetings 'all the time' and filling out clinical incident forms when they felt patient safety had been compromised as a consequence of the coordinator's workload. However they felt that minimal attention had been paid to this issue. On review of five sets of ward meeting minutes (dated March, July, August, September, November, 2009) the issue of coordinators was raised twice ( March and November). In March there was a brief one line entry regarding coordination which states that coordinators should take only three patients. In November there was a longer entry which describes that coordinators take a complex load, answer the phone, and manage the ward. The entry states that coordinators should be allocated a fair patient load or not have a load at all. There is also mention of the possibility of a budgeted full time coordinator for a trial period, although there are no details recorded regarding when this might start. 


\subsubsection{Summary}

The domains to emerge from the interview and focus group data for Medical ward $\mathrm{C}$ were healthcare workforce and organisation. Table 32 presents the factors participants believed most affected care of acutely unwell ward patients on Medical ward C.

Table 32. Domains, themes, and factors Medical ward C

\begin{tabular}{|l|l|}
\hline Workload & Healthcare workforce \\
& Having time and coping \\
& Task focus \\
& Nurse to patient ratio \\
& Skill mix \\
\hline Teamwork & Support \\
& Clinical experience \\
\hline Communication & Responding \\
& Breakdown in communication \\
\hline \multicolumn{2}{|c|}{ Organisation } \\
\hline Leadership, accountability and roles & Leadership roles \\
\hline
\end{tabular}

\subsection{SURGICAL WARD D}

\subsubsection{Setting the scene}

At the time of the study the surgical ward was a 27-bedded area. The ward was divided into four-bedded bays and single rooms. There was a designated 'step down' area where patients who had higher acuity levels were nursed. Four different teams admitted patients to the ward. These were general/colorectal; specialty breast and endocrine; general surgery/gastro/liver and general surgery trauma/breast. The ward took very few outliers as their occupancy was high. The teams of surgeons who admitted patients to the ward were colour coded. Patient notes were kept in colour coded trolleys in the doctors' room and it was the medical staff's responsibility to ensure they were stored in the correct location.

On the ward there was also a large staff room with two sofas, surgical meeting room, interview room, CNM office and a CNE office. The ward was undergoing refurbishment to some of the communal areas at the time of the study. This included some of the bathrooms and the patients' day room, and a separate meeting room. 


\section{Ward personnel}

The ward was budgeted for 27.2 FTE. This equated to 44 members of staff which demonstrates a high number of part time staff. The CNM was accommodating regarding flexible employment contracts for staff with children or other care commitments, although the minimum contract was for 0.4 FTE. At the time of the study there were no nursing vacancies, but there were vacancies for 1.4 FTE HCA. The CNE was full time and worked between two surgical wards ( $0.5 \mathrm{FTE}$ per ward). The CNM encouraged staff to socialise together. There were several staff functions a year and medical staff were invited and usually attended.

The roster was split in to three sections: senior, intermediate, and junior. The rostering was done by the $\mathrm{CNM}$. The $\mathrm{CNM}$ tried to ensure that there were at least two seniors on per shift. To be senior, staff needed to have completed their intravenous drug administration (IV) certification, epidural, patient controlled analgesia (PCA) certification and have coordination experience. The ward was coordinated by the CNM Monday to Thursday on the morning shift and she did not take patient load. When the CNM did not coordinate the morning shift, the ward was budgeted for a coordinator who also did not take a patient load. On the afternoon and night shifts the coordinator was the most senior person on the shift and took a patient load which, theoretically, was lighter. In reality this did not always manifest, as the coordinator often took the sickest patients on the ward if they had concerns regarding the skill levels of the staff on the shift. There was no formal training for the staff who took on the coordination role, although there was a resource file available on the ward which contained useful information.

A ward clerk worked from 0730 - 1600 Monday to Friday and $0730-1000$ Saturday and Sunday. There was no patient communication system. The CNM had been requesting a system for the last two years, but this request had been denied by the operations manager due to budgetary restraints.

Staff on long-term sickness was minimal (0.3 FTE). Staff were encouraged to take annual leave and 2.8 FTE were allowed on annual leave at any one time. There were no large outstanding leave balances. Staff turnover was minimal. 
Nursing handover was given by the coordinator. There was a computerised handover sheet which staff updated prior to handover. There was a global snapshot handover in the office and then a bedside handover. This was a fairly recent change, which all staff were not compliant with. Staff were allocated their patient load by the coordinator.

The ward had recently been following the principles of the Productive Ward, which had been championed by the CNM and CNE. Staff were actively encouraged to contribute ideas which would make the ward a better place to work in. This had seen the storage and sluice areas reorganised and some better signage for the ward.

\section{Patient Acuity Tool}

The ward has an acuity tool which staff used to document patient acuity data on every shift.

\subsection{Surgical ward D: Within-case analysis}

The domain which emerged from the interview and focus group data for Surgical ward D was healthcare workforce. The main themes to emerge from healthcare workforce were workload, teamwork, communication, and leadership, roles and responsibilities. The title and number of participants who took part in interviews or focus groups are listed in Table 33.

\section{Table 33. Participants from Surgical ward D}

Surgical Nurses $\times 2$ (FGNursesSurgx2H2)

Clinical Nurse Educator (CNESurgH2)

Clinical Nurse Manager (CNMSurgH2)

House Surgeon Surgical (HSSurgH2)

Surgical Nurse 1 (SurgNurs1H2)

Surgical Nurse 2 (SurgNurs2 2 2)

(Letters in parenthesis are code names from participant transcripts)

In addition to interview and focus group data, documentation pertaining to the care of acutely unwell ward patients was reviewed and is presented as Table 34.

\section{Table 34. Documents reviewed for Surgical ward D}

Ward meeting minutes March - November 2009

Ward Nursing Rosters 8 weeks October/November

Acuity Tool Surgical Floor 


\subsubsection{Teamwork}

Factors affecting teamwork were discussed by both nursing and medical participants from this ward. According to the participants, relationships were very important to successful team working. Nursing participants had mixed views regarding the success of relationships within the team. For some nursing participants relationships between doctors and nurses were viewed as hierarchical at times.

"Other specialties are more collegial, general surgery in particular I think is really hierarchical and that's not helpful"

(FGNursesSurgx2H2)

Nursing participants viewed this hierarchy as having a negative impact on patient care as they felt that their opinion was regarded as either not valid or required. The CNM recounted a scenario where a patient had deteriorated and she had contacted one of the surgical consultants. On review of the patient the consultant had dismissed her concerns. However, the CNM was sufficiently worried to bypass the consultant and insist that the house surgeon refer the patient to ICU. The patient was seen by the ICU consultant and was transferred to ICU immediately.

Was there any follow up to that, was there any discussion with him after? SQ

"Yep I did discuss with him and I got, well you're wrong. She must have had a rapid deterioration after the time that I saw her and basically it was like at the end of the day my little voice as a senior nurse was just wiped, it's not going to happen yeah. Which is really frustrating" (CNMSurgH2)

The CNM believed that when the registrars and consultants consulted the nursing staff regarding patient care and asked their opinion this made a huge difference to the effectiveness of team working. Although the CNM found some situations frustrating, she was not daunted by having to voice her concerns regarding patient care or about medical staff behaviour. However there was no formal forum for the senior medical or nursing staff to get together and discuss any issues affecting the team.

"We used to be, both charge nurses and the booking clerks and the educator used to prior to this new director used to be invited to the monthly meetings with the operations manager of the service as 
well. But that for the last year that hasn't happened. It's just like for instance now that we've got a different person organising the Thursday meetings that the nurses are no longer invited which is something that we're working to change but at the moment it's been so difficult probably in the last six months that we've decided as a team to wait until the next lot of registrars start" (CNMSurgH2)

When the director of surgery had concerns he approached the CNM.

"I must admit that the director does, like if he senses issues himself he'll come and, like he'll say can I have ten minutes [NAME REMOVED] or half an hour and he'll just ask me what my thoughts are, feelings are about certain things especially if he's picked up anything but we don't have that formal voice" (CNMSurgH2)

Nursing participants believed some of the difficulties associated with team working were associated with credibility and trust. Nursing participants believed that if the doctor they were dealing with felt the nurses were sufficiently experienced the doctor trusted their judgement much more and would respond in a more appropriate way. For example if the CNE or the CNM phoned and asked a doctor for an urgent patient review then the doctor would come immediately. The CNM found this frustrating, as she believed it devalued her staff. The nursing participants shared a similar view.

"I think it's more the fact that they know, they know she knows what she's talking about. I mean she's been probably in surgical nursing for 20 years or probably even longer but they will know that she knows her job whereas they're saying to us well who are you, you're just a nurse I'm a house surgeon I know what I'm talking about" (NursSurg1H2)

However, trust between medical and nursing staff was a two-way issue, as nursing staff also took time to trust the junior doctors.

"And if you have got something serious they might not believe you. I'm fairly experienced, I'm not kind of green. I mean we see teams and teams of house surgeons coming through. We often take a while to trust them because you do get the odd house surgeon that doesn't take things seriously" (NursSurg2H2)

Nursing participants believed that some of the house surgeons' hesitance in dealing with acutely unwell ward patients was also due to their inexperience and a lack of 
confidence. Furthermore it was also due to a lack of support as more senior doctors were busy all day in theatre.

"And I just put it down to the house surgeon being uncomfortable with sick patients. And that's, that's probably they just feel unsupported themselves. So they tend to kind of steer away and hope the thing fixes itself and when they come back things will all be better. It doesn't work that way" (CNESurgH2)

Senior nursing participants described supporting the junior doctors when patients became acutely unwell. In return, the nursing participants expected to be acknowledged and respected for that support and to be listened to when they raised concerns. The CNM believed that the effectiveness of the relationships was variable; nevertheless she encouraged the staff to persevere.

The ward was a supportive area to work in from the nursing participants' perspective. The CNM stated that in staff meetings she encouraged staff to work as a team, to look out for each other and offer to help if they saw someone struggling. Nursing participants believed that there was a positive team culture within the ward.

"And make sure that they're feeling supported so that they can come and ask you for your help. I always ask them, I always ask you know how you're going. How's so and so or I think it's, it's quite good here where you know it's quite open for people to talk about things so it's quite a nice, well you don't hear any real bitching and moaning it's all pretty positive stuff" (NursSurg2 $\mathrm{H} 2$ )

One nursing participant described the ward team as having a 'nice sort of camaraderie', which was important because issues with workload often meant they needed to rely on one another.

\subsubsection{Workload}

Nursing participants described the ward as very busy and 'fast paced'. This was due to patient acuity and rapid turnover of patients. The ward received most of the surgical discharges from the High Dependency Unit (HDU) and had developed a 'step down' area in which to care for them. These patients were perceived by nursing participants as being very high acuity patients. The CNM encouraged staff 
to enter acuity data regarding their patients and the ward's high patient acuity levels had recently been acknowledged by the organisation.

"it's been quite good because it's been recognised with putting all our numbers into One Staff that our acuity is high and we've actually got two further FTE from it which means we are able to now have a senior staff nurse from nine o'clock to two" (CNMSurgH2)

However, having time for all their patients was viewed as challenging by participants, particularly if their patient load included an acutely unwell patient.

"If you've got a really acutely unwell patient with an incredibly high workload then you won't do the housekeeping stuff that you would have liked to have done and your well stable patients are not getting the hours and the attention that they should have because it's all going into a couple of other patients" (FGNursesSurgx2H2)

Nursing participants felt conflicted regarding which patient should be their priority. Nursing participants described some days as 'rubbish' or as having done 'a dreadful job today' because of having to prioritise. Staff did not get meal breaks and they often left work late because they needed to stay on to complete their patient care. Some nursing participants believed that the heavy workload also impacted on participants' health and wellbeing.

"But I struggled for two more days after that and then I couldn't, I couldn't put one more foot in front of the other so I had to ring in sick. And that was a consequence of that workload and that's what happens, that's literally what happens" (FGNursesSurgx2H2)

However, nursing participants described allocation of patients as fair and flexible. If staff were allocated a high acuity patient then they would be given a smaller patient load, providing the skill mix and staffing numbers allowed for that.

Both nursing and medical participants discussed the heavy workload of medical staff. Surgical registrars were in the operating theatre all day, which left the house surgeons to cover the wards. This meant that they also needed to prioritise their workload. The ability to prioritise their workload was very dependent upon the information they received from the nursing staff and the communication that went on between them. 


\subsubsection{Communication}

Participants stated that a breakdown in communication between medical and nursing staff was often due to the amount of information the house surgeons received when being asked to urgently review a patient. The medical participant believed that sometimes they were not given sufficient information to be able to prioritise the nursing requests for patient review.

"And it's a bit annoying sometimes when they say oh I think you need to review this patient and you go why and they say oh they've become unwell. What are the obs.? Oh I don't know, the person looking after her just asked me to call you and then you don't know what to expect. There's not enough information so it might be a sick patient but because they will probably be put as a lower priority because it doesn't sound too bad, yeah. So I guess if there's adequate information it will help us to decide who to see first and, and because we are usually quite busy and time is quite pushed so if we can have enough information to help us decide who to see first, which one to go to first then that would help" (HSSurgH2)

Conversely, the nursing participants described not being listened to even when they used SBAR to request an urgent review. Nursing participants expressed the view that they needed to be articulate and thorough in order to get the required response, but felt frequently frustrated when their requests for review were ignored. Furthermore, nursing participants felt that junior doctors were often reluctant to refer patients to their registrars or other specialties such as ICU because of the response they would receive.

"That happens probably 50\% of the time if I think back on all the incidents I've had, $50 \%$ of the time you're struggling with junior medical staff who can't take the appropriate action or don't know how to escalate or try to escalate and get beaten down by their registrars" (FGNursesSurgx2H2)

When this occurred senior nursing participants felt confident enough to contact the registrar or consultant themselves; however, these participants believed that this was not the case with junior or intermediate nurses and this was therefore a factor which affected care of acutely unwell ward patients. 


\subsubsection{Leadership, accountability and roles}

The main issue for participants within this theme was leadership roles, specifically the inability of the coordinator to provide effective shift leadership due to an excessive workload. On an afternoon and night shift the coordinator was the most senior nurse on the shift. If the staffing levels were optimal the coordinator was supposed to allocate herself a lighter load. However participants stated that in reality this did not happen. Nursing participants believed that this was a dangerous scenario.

"In the worst case scenario here you could have a really heavy load and coordinate and that is dangerous for the other patients because if you have a junior nurse who's got too heavy a load, or anybody who's got too heavy a load they don't pick up on the signs of deterioration and therefore they don't report them to the coordinator. The coordinator's too busy with their own workload they're not aware of deteriorations happening in other rooms with other staff. That's another reason for the management not being optimal" (FGNursesSurgx2H2)

Nursing participants believed that the coordinator's ability to have an overview of the ward was severely compromised by them carrying such a heavy patient load. However, the CNM believed that the senior nurses who coordinated often gave themselves an unreasonable workload because they liked looking after sick patients, and not only because there was no one else sufficiently experienced on the shift. She also believed that the senior nurses needed to encourage junior staff to take high acuity patients with the support of more senior nurses, so that they could develop the appropriate skills. When coordinators took high acuity patients and were busy, ward management tasks were not done and junior staff were not supported.

\subsubsection{Summary}

The only domain to emerge from the interview and focus group data for Surgical Ward D was healthcare workforce. Table 35 presents what the participants believed were the factors most affecting the care of acutely unwell ward patients on the Surgical ward D. 
Table 35. Domains, themes, and factors Surgical ward D

\begin{tabular}{|l|l|}
\hline \multicolumn{2}{|c|}{ Healthcare workforce } \\
\hline & Relationships \\
& Support \\
& Credibility and Trust \\
& Clinical experience \\
& Being confident \\
\hline Workload & Having time and coping \\
& Task focus \\
& Allocation \\
\hline Communication & Responding \\
& Breakdown in communication \\
\hline Leadership, accountability and roles & Leadership roles \\
\hline
\end{tabular}

\subsection{Summary}

This chapter has provided a detailed description of each case and the hospital context within which the case sits. In addition the findings of the within-hospital and within-case analyses are presented which have demonstrated key themes and factors. In order to determine whether factors affecting the care of acutely unwell ward patients are specific to particular wards or common across organisations it is necessary to carry out a cross-case analysis. This is presented as Chapter 6. 


\section{CHAPTER 6: CROSS-CASE AND CROSS-HOSPITAL ANALYSIS}

\subsection{Introduction}

Chapter 5 presented both a description and within-case analysis of all four cases and a description and within-hospital analysis of Hospital 1 and Hospital 2. This stage of the analysis identified key themes and factors specific to each case and each hospital. Whilst it is important to determine factors specific to each case and hospital, the findings of this study will be strengthened if common factors are identified across all cases and both hospitals. Similarly, if differences emerge, explanations can be sought as to why these have occurred. Conclusions can then be drawn as to the effect of the context in relation to these similarities and differences. In order to determine whether there were any common factors across cases and across hospitals, a cross-case and a cross-hospital analysis were performed.

This chapter will present the results from the cross-case analysis and the cross-hospital analysis. Using four additional questions (see section 3.11.3) to guide the cross-case and cross-hospital analysis, this chapter is divided into two sections.

The first section presents a cross-case analysis. The purpose of the analyses was to determine if similarities and differences in factors that affect the care of acutely unwell ward patients existed between individual cases.

The second section of this chapter presents a cross-hospital analysis. As is recognised by the case study approach, the context is often difficult to separate from the phenomenon. This comparison of the two contexts allowed the identification of patterns and themes across both hospitals. These patterns and themes were then compared with the themes that emerged from the cross-case analysis. By separating the analysis into case and hospital sections the similarities and differences between case and organisational viewpoint regarding factors affecting care of acutely unwell ward patients could also be illustrated.

\subsection{The cross-case analysis}

Cross-case analysis allows the researcher to derive patterns or differences between cases. When relationships and explanations across the data have been 
determined conclusions can be drawn (Miles \& Huberman, 1994). This increases both the strength and analytic generalisability of the findings (Yin, 2003). Common themes that emerge can then become the basis of recommendations for change.

One way of analysing the data to determine these patterns or differences is through the construction of matrices. Matrices provide a systematic comparison which allows the researcher to see "sharply whether and where a pattern dominates" (Richards, 2009, p. 183). Key themes and factors were extracted from the within-case analyses of all four cases. These key themes and factors were then mapped against the analytical framework (Table 17) to form the cross-case analysis matrix. Table 36, the cross-case matrix, demonstrates the findings of the cross-case analysis. In the process of the cross-case and cross-hospital analysis both key and minor themes emerged. The term' key theme' is used to describe themes which were discussed the most by participants. Minor themes reflect those themes least discussed.

The symbols $(+,-)$ used in the matrix are a way of demonstrating the orientation of the discussion related to the key theme i.e. discussed in either a positive or negative way. Having a double plus or double negative symbol indicates sufficient discussion by participants to describe the theme as a key theme. A single plus or negative sign denotes a minor theme.

\section{Table 36. Cross-case matrix}

\begin{tabular}{|c|c|c|c|c|}
\hline THEME/FACTOR & Wa & Wa & Wa & \\
\hline Workload & & & & \\
\hline Having time and coping & -- & -- & -- & -- \\
\hline Task focus & - & - & -- & - \\
\hline Allocation & - & - & $x$ & ++ \\
\hline Nurse to patient ratio & -- & -- & -- & - \\
\hline Skill mix & -- & - & -- & - \\
\hline Teamwork & & & & \\
\hline Relationships & - & ++ & - & +1 \\
\hline Support & $+/-$ & ++ & ++ & ++ \\
\hline Credibility and Trust & - & $x$ & - & -- \\
\hline Clinical experience & - & -- & -- & -- \\
\hline Being confident & - & $x$ & - & - \\
\hline
\end{tabular}


Table 36 (continued). Cross case matrix

\begin{tabular}{|c|c|c|c|c|}
\hline \multirow{2}{*}{ THEME/FACTOR } & \multirow{2}{*}{\multicolumn{4}{|c|}{ Ward A Ward B }} \\
\hline & & & & \\
\hline Responding & - & -- & -- & - \\
\hline Breakdown in communication & -- & -- & -- & - \\
\hline \multicolumn{5}{|l|}{ Leadership, Accountability and Roles } \\
\hline Leadership roles & - & -- & -- & - \\
\hline Being accountable & - & - & - & - \\
\hline Seeing the bigger picture & - & $x$ & - & $x$ \\
\hline Roles of doctors and nurses & - & $x$ & - & $\mathrm{x}$ \\
\hline \multicolumn{5}{|l|}{ Organisation of $24 / 7$ services } \\
\hline Support & - & - & - & - \\
\hline Continuity of care & - & - & - & $\mathrm{x}$ \\
\hline \multicolumn{5}{|c|}{ Organisational systems and processes of care } \\
\hline Service initiatives & + & ++ & - & + \\
\hline Organisational policies & - & -- & - & - \\
\hline Access & - & - & - & - \\
\hline \multicolumn{5}{|l|}{ Culture and Environment } \\
\hline Ward culture & - & - & + & $\mathrm{x}$ \\
\hline Organisational culture & - & - & - & $x$ \\
\hline Nurses' cultural background & - & - & - & - \\
\hline Interactions with families & - & - & $x$ & $x$ \\
\hline Ward layout & - & $x$ & $x$ & - \\
\hline \multicolumn{5}{|l|}{ Skills and Knowledge deficit } \\
\hline Workforce preparation & - & $x$ & $x$ & - \\
\hline Assessment skills & - & - & - & - \\
\hline Prioritising & - & - & - & $x$ \\
\hline Escalating care & - & - & - & - \\
\hline Using machinery & - & $x$ & $x$ & $x$ \\
\hline \multicolumn{5}{|l|}{ Opportunities for learning } \\
\hline Bedside learning & - & $x$ & $x$ & - \\
\hline Receptiveness to training and education & - & $x$ & $x$ & $\mathrm{x}$ \\
\hline
\end{tabular}

Key to cross-case analysis matrix table

$\begin{array}{ll}-- & \text { Discussed as a key theme from a negative perspective } \\ - & \text { Discussed as a minor theme from a negative perspective } \\ ++ & \text { Discussed as a key theme from a positive perspective } \\ + & \text { Discussed as a minor theme from a positive perspective } \\ +/- & \text { Discussed as a key theme from both a positive and negative perspective } \\ \mathrm{X} & \text { Did not emerge as a theme in this case }\end{array}$

The cross-case matrix (Table 36) was then used to answer the following questions: 
1. Do the factors that affect the care of acutely unwell ward patients vary within medical and surgical wards within Hospital 1 and Hospital 2?

2. Are there any similarities and differences in factors between Medical ward A and Medical ward C?

3. Are there any similarities and differences in factors between Surgical ward B and Surgical ward D?

4. Are there any similarities and differences in factors between medical and surgical wards across both organisations?

5. Are there any similarities and differences in factors between Hospital 1 and Hospital 2 from a wider organisational perspective?

The similarities and differences which became apparent from the cross-case analysis are presented as Tables 37 to 42 . The key themes are presented in the tables and then discussed in order of the weight of the data associated with that theme.

\subsection{Analysis of similarities and differences between Medical ward $A$ and Surgical ward B in Hospital 1}

Using the findings from the cross-case analysis presented as Table 36, the key themes and factors were extracted and used to determine the similarities and differences between Medical ward A and Surgical ward B. These findings are illustrated in Table 37.

Table 37. Similarities and differences in factors between Wards A and B within Hospital 1

\begin{tabular}{|l|l|l|}
\hline \multicolumn{3}{|c|}{ SIMILARITIES } \\
\hline THEME/FACTOR & Ward A & Ward B \\
\hline Workload & \multicolumn{2}{|l|}{} \\
\hline Having time and coping & -- & -- \\
\hline Nurse to patient ratio & -- & -- \\
\hline Teamwork & \multicolumn{2}{|}{} \\
\hline Support & + -- & ++ \\
\hline Clinical experience & -- & -- \\
\hline
\end{tabular}


Table 37 (continued). Similarities and differences in factors between Wards A and B within Hospital 1

\begin{tabular}{|c|c|c|}
\hline \multicolumn{3}{|c|}{ SIMILARITIES } \\
\hline THEME/FACTOR & Ward A & Ward B \\
\hline \multicolumn{3}{|l|}{ Communication } \\
\hline Responding & -- & -- \\
\hline Breakdown in communication & -- & -- \\
\hline \multicolumn{3}{|c|}{ DIFFERENCES } \\
\hline THEME/FACTOR & Ward A & Ward B \\
\hline Workload & & \\
\hline Allocation & - & -- \\
\hline Teamwork & & \\
\hline Relationships & - & ++ \\
\hline Skill mix & -- & - \\
\hline $\begin{array}{l}\text { Leadership, Accountability and } \\
\text { Roles }\end{array}$ & & \\
\hline Leadership roles & - & -- \\
\hline $\begin{array}{l}\text { Organisational systems and } \\
\text { processes of care }\end{array}$ & & \\
\hline Service initiatives & + & ++ \\
\hline Organisational policies & - & -- \\
\hline \multicolumn{3}{|l|}{ Key } \\
\hline \multicolumn{3}{|c|}{ Discussed as a key theme from a negative perspective } \\
\hline Discussed as a minor $\mathrm{t}$ & me from a & ve perspective \\
\hline Discussed as a key the & e from a pc & perspective \\
\hline Discussed as a minor $\mathrm{t}$ & me from a & e perspective \\
\hline Discussed as a key the & e from bot & itive and negative perspective \\
\hline Did not emerge as a th & me in this & \\
\hline
\end{tabular}

Table 37 demonstrates that the key themes to emerge in this comparison are workload, teamwork and communication, leadership, accountability and roles, and organisational systems and processes of care.

\subsubsection{Workload}

Participants from both wards described busy ward environments with heavy workloads. The difficulties participants faced with these heavy workloads led to feelings of dissatisfaction and distress. Nursing participants believed that they did not have time to care for acutely unwell ward patients and adequately take care of 
the rest of their patient load. Nursing participants from both wards suggested that asking for help from other staff was difficult since everyone was equally busy. In both wards, being short staffed affected the nurse to patient ratio which in turn increased their workload, ultimately affecting their ability to care for acutely unwell ward patients.

There were several differences within the theme of workload between Medical ward A and Surgical ward B. Participants from Medical ward A highlighted skill mix as a challenging factor. According to the roster on Medical ward A there were 19 staff who had been designated as senior within the ward; however, participants described poor skill mix as one of the key factors affecting care of acutely unwell ward staff. Additional nursing roles such as the ACNM and 0.5 CNE sat within the senior section of the roster. These roles would not be included in the numbers of nurses rostered on per shift. The impact of the lack of senior staff was demonstrated by the fact that the initial collaborative model of care had to be abandoned due to lack of experienced staff. Therefore there is disparity between the roster designation of senior and what the nursing participants perceived as senior staff available to participate in patient care /presence of senior staff on the ward.

In contrast, in Surgical ward B nursing participants described a core of very senior nurses who had worked on the ward for many years. This may explain why skill mix did not feature as such an issue for this ward. Within Surgical ward B there was an emphasis placed on getting to know work colleagues socially in order to facilitate better teamwork, which was not apparent within Medical ward A. Nursing participants believed that this had led to the retention of staff and the presence of a core of senior, experienced staff. This may also explain why nursing participants from Surgical ward B believed developing relationships between staff was so important.

Allocation of patients was discussed on Surgical ward B but did not emerge as a key issue on the medical ward. On Surgical ward B patients were allocated to the nurses of the oncoming shift by the preceding shift coordinator. Nursing participants thought this was an issue, as over the course of a shift patients' 
conditions could change, altering workload significantly. Consequently nursing participants stated that the preceding shift coordinator may not be sufficiently up to date with the patients' condition and therefore workload allocations may be unrealistic. Nursing participants believed that this impacted on their ability to safely provide all the care that their patients (acutely unwell or stable) required.

More senior nursing participants described approaching the coordinator to have their patient allocation changed; however, they believed junior staff and staff new to the ward may not have the confidence to do this. Consequently, these less experienced staff would struggle on, barely coping, leading some nursing participants to believe there was the possibility that patients who were acutely unwell would not receive the attention that was required.

\subsubsection{Teamwork}

Having a team which supported each other and who were able to ask each other for help was important to nursing participants from both wards. On the whole nursing participants believed that their wards were supportive environments. Nursing participants felt that the culture of their ward allowed them to ask for help, but this rarely occurred because they believed that everyone was equally busy and they didn't want to add to their colleagues' workload.

Having sufficient numbers of clinically experienced nursing staff within the team was important to ensure that acutely unwell ward patients were cared for appropriately. In addition, having that clinical experience recognised and validated was also seen as important by nursing participants. When doctors did not recognise nurses' clinical experience, this had a detrimental effect on teamwork as nurses felt resentful at having to work with medical staff who, they felt, did not value their expertise.

Furthermore nursing participants talked of being frustrated by having to justify their assessments of acutely unwell ward patients. This was not only wearisome but took up valuable time. Conversely senior, more experienced nurses were happy to support more junior medical staff when this was required, with some senior nursing participants believing this to be a valid aspect of their role. 


\subsubsection{Communication}

According to nursing and medical participants from both wards breakdown in communication was an important factor which affected the care of acutely unwell ward patients. Nursing participants described difficulties obtaining a response from medical staff and medical participants were frustrated at times by the limited amount of information they were given when nursing staff referred patients. This was despite the introduction of a computerised communication system (CCS) and the SBAR framework.

Within Medical ward A communication at handover between nursing staff was highlighted as a negative factor. This was due to the way in which handover was conducted using a Dictaphone. Using the Dictaphone meant there was no opportunity to ask questions or clarify issues related to patients' conditions. Nursing participants on Medical ward A believed that important details could be missed due to tiredness. Having a disembodied voice rather than an actual person delivering the handover limited opportunities for clarification. However, despite these limitations being highlighted as an issue from both a ward and senior management perspective, there were no plans to change the handover process.

In contrast, on Surgical ward B handover was given by the coordinator of each shift. Having the coordinator in the room delivering the handover facilitated discussion regarding some patients' conditions and allowed any queries to be answered there and then.

\subsubsection{Leadership, accountability and roles}

Leadership roles, specifically the failure of the coordinator to provide shift leadership due to a perceived excessive workload, was a key theme to emerge from Surgical ward B. On Surgical ward B the coordinator had a full patient load as well as responsibility for coordinating the ward. This was not the case in Medical ward A. Organisational attention had been focused on Medical ward A after the occurrence of a serious and sentinel event. Surgical ward B had not received the same level of attention. The organisation had recognised on Medical ward $A$ that it was necessary to provide a coordinator who did not carry a patient load. As a 
consequence, the difficulties associated with taking a patient load and coordinating the ward were not as significant for Medical ward A nursing participants.

The disparity in staffing levels between the medical and surgical ward which allowed a coordinator without a patient load was raised as an equity issue by the CNM of the surgical ward. Given that the organisation believed that having a coordinator without a patient load was beneficial to one area, it is unclear why this was not hospital-wide policy.

\subsubsection{Organisational systems and processes of care}

Within the theme of organisational systems and processes of care, service initiatives, specifically the critical care outreach service (CCOS) and the Early Warning Score (EWS), were described as positive initiatives which assisted and supported nurses in caring for acutely unwell ward patients. Despite the CCOS and EWS being hospital-wide service initiatives, these initiatives did not come across as major discussion points within the interviews and focus groups on Medical ward A. This is interesting, given that according to the 2009 CCOS annual report Medical ward $\mathrm{A}$ was the most frequent user of the service.

Nursing participants from Surgical ward B also described organisational policies, specifically the management of medical outliers, as a factor which affected the care of acutely unwell ward patients. According to nursing participants caring for outliers was difficult as some aspects of care were unfamiliar to surgical nurses. Furthermore nursing participants described difficulties in knowing the correct medical staff to contact about plans of care. According to nursing participants finding the correct doctor and determining how to care for these outliers was time consuming and impacted on the time available to care for acutely unwell patients.

Nursing participants from Medical ward A did not perceive the management of outliers as a factor which affected the care of acutely unwell ward patients. An explanation for this may be that surgical wards are more likely to care for medical outliers due to medical wards having less patient turnover than surgical wards. Surgical patients are also more likely to have a more predictable length of hospital stay than medical patients, as more patients are admitted for elective procedures. Conversely, there are very few elective admissions to medical wards and most 
patients are admitted as emergencies. When a medical ward is full, patients admitted via the emergency department are admitted to any free bed within the hospital and this is invariably a surgical bed. Therefore it is highly unlikely that there would be surgical outliers on medical wards. Hence, medical nurses would have very limited experience of caring for surgical outliers.

\subsection{Analysis of similarities and differences between Medical ward C and Surgical ward D in Hospital 2}

Using the findings from the cross-case analysis presented as Table 36, the key themes and factors were extracted and used to determine the similarities and differences between Medical ward C and Surgical ward D. These findings are illustrated in Table 38.

Table 38. Similarities and differences in factors between Wards $C$ and $D$ within Hospital 2

\begin{tabular}{|c|c|c|}
\hline \multicolumn{3}{|c|}{ SIMILARITIES } \\
\hline THEME/FACTOR & Ward C & Ward D \\
\hline \multicolumn{3}{|l|}{ Workload } \\
\hline Having time and coping & -- & -- \\
\hline \multicolumn{3}{|l|}{ Teamwork } \\
\hline Support & ++ & ++ \\
\hline Clinical experience & -- & -- \\
\hline \multicolumn{3}{|l|}{ Communication } \\
\hline Responding & -- & -- \\
\hline Breakdown in communication & -- & -- \\
\hline \multicolumn{3}{|l|}{$\begin{array}{l}\text { Leadership, Accountability and } \\
\text { Roles }\end{array}$} \\
\hline Leadership roles & -- & -- \\
\hline \multicolumn{3}{|c|}{ DIFFERENCES } \\
\hline THEME/FACTOR & Ward C & Ward D \\
\hline \multicolumn{3}{|l|}{ Workload } \\
\hline Allocation & $\mathrm{x}$ & ++ \\
\hline Task Focus & -- & - \\
\hline Nurse to patient ratio & -- & - \\
\hline Skill mix & -- & - \\
\hline \multicolumn{3}{|l|}{ Teamwork } \\
\hline Relationships & - & $+/-$ \\
\hline
\end{tabular}




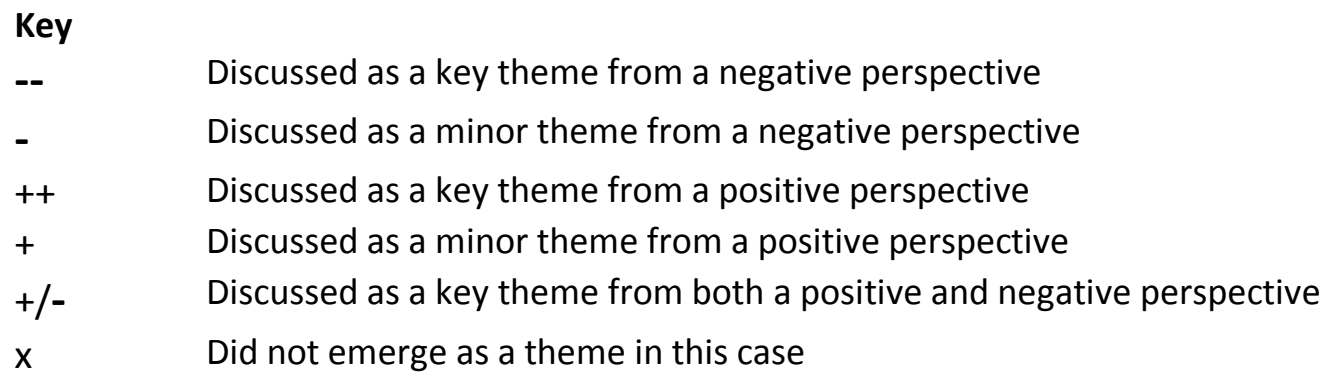

Table 38 demonstrates there were more similarities than differences between the medical and surgical wards studied in Hospital 2 in terms of factors affecting care of acutely unwell ward patients. The consistent themes which emerged included workload, teamwork, communication, and leadership, accountability, and roles.

\subsubsection{Workload}

Within the theme of workload there were similarities and differences in factors. Nursing and medical participants from both wards identified that having time and coping with the ward workload was difficult. This was due to both the complexity and number of patients that participants cared for. Nursing participants stated they did not have enough time to adequately care for patients and had to prioritise between patients, which left them feeling distressed. Medical participants described feeling pressurised by the number of patients they were required to manage when on call and after hours.

Within Medical ward $\mathrm{C}$ nurse to patient ratio was identified as an issue that affected workload, with staff shortages increasing the number of patients each nurse had to care for. Nursing participants described staff becoming task focused, concerned with completing routine 'jobs' as opposed to providing individualised nursing care to patients as a way of coping with heavy workloads and staff shortages. However this was not identified as a key factor in Surgical ward D. Medical ward C appeared to have problems ensuring adequate staffing numbers; this issue was not as apparent on Surgical ward D. Surgical ward D had utilised patient acuity data to secure additional staffing resources. Medical ward C did not collect acuity data.

Skill mix was also identified as a factor on Medical ward C. Nursing participants described shifts where the majority of staff were very inexperienced, 
which left nursing staff feeling unsupported. This may be because of the staffing issues previously alluded to. Surgical ward D had a core of senior staff who had worked on the ward for many years, which again was not the case with Medical ward C.

\subsubsection{Teamwork}

Within the theme of teamwork, participants from both wards in Hospital 2 described support as important when caring for acutely unwell ward patients. Nursing participants from both wards described ward environments where nurses were supported by each other but usually only when they asked for help. The level of clinical experience of both nursing and medical staff also impacted on the care of acutely unwell ward patients. Nursing participants from both wards described scenarios where inexperienced staff (nursing and medical) struggled on with their workload and did not ask for help. Conversely nursing participants also described scenarios where senior, experienced nursing and medical staff made faster and more accurate assessments, prioritised their workloads more effectively and got action more quickly for their acutely unwell ward patients.

In terms of differences between the wards in Hospital 2, nursing participants from Surgical ward D placed a great deal of importance on relationships to enhance teamwork. Within Surgical ward D the perception of (at times) challenging hierarchical relationships between doctors and nurses were more apparent than in Medical ward C. However on Surgical ward D there appeared to be an investment from the Clinical Nurse Manager (CNM) in developing relationships between nursing staff in social activities outside work which was not described by participants within Medical ward C. The investment in relationships and the core of senior staff on Surgical ward D may also have accounted for the positive attitudes regarding patient allocation on Surgical ward D. Nursing participants on Surgical ward $D$ were confident that, if they were allocated a heavy load that they felt was inappropriate, they could either ask to change or, if they were junior, someone senior would intervene on their behalf. 


\subsubsection{Communication}

Breakdown in communication was a key factor which nursing and medical participants on Medical ward C and Surgical ward D stated affected the care of acutely unwell ward patients. Responding to requests for patient review and poor communication between nursing and medical staff were key factors from both wards. Nursing participants described situations where they would make requests for urgent review that were not responded to in a timely or appropriate manner. Medical participants were frustrated at the amount and accuracy of patient information that they were given. This was despite the implementation of the SBAR framework at Hospital 2.

\subsubsection{Leadership, accountability and roles}

Leadership roles, specifically the multiple responsibilities and perceived excessive workload of the coordinator, emerged as an important factor for nursing participants from both wards. The fact that the coordinator was required to take a full patient load and carry the additional responsibilities associated with coordinating the ward was identified as a factor that detrimentally affected the care of acutely unwell ward patients. Nursing participants from both wards described the difficulties of the coordination role, especially when patients were acutely unwell. Coordinators were usually the most senior nurse on the shift and therefore would allocate the sickest patients to themselves, and were therefore not available to support other staff members or have 'peripheral vision' for the rest of the ward because of their own workload.

\subsection{Analysis of similarities and differences between Medical ward A and Medical ward C}

Using the findings from the cross-case analysis presented as Table 36, the key themes and factors were extracted and used to determine the similarities and differences between Medical ward A and Medical ward C. These findings are illustrated in Table 39. 
Table 39. Similarities and Differences between Medical ward A and Medical ward C

\begin{tabular}{|c|c|c|}
\hline \multicolumn{3}{|c|}{ SIMILARITIES } \\
\hline THEME/FACTOR & Ward A & Ward C \\
\hline \multicolumn{3}{|l|}{ Workload } \\
\hline Having time and coping & -- & -- \\
\hline Nurse to patient ratio & -- & -- \\
\hline Skill mix & -- & -- \\
\hline \multicolumn{3}{|c|}{ SIMILARITIES } \\
\hline THEME/FACTOR & Ward A & Ward C \\
\hline Teamwork & & \\
\hline Support & $+/-$ & ++ \\
\hline Clinical experience & -- & -- \\
\hline Communication & & \\
\hline Responding & -- & -- \\
\hline Breakdown in communication & -- & -- \\
\hline \multicolumn{3}{|c|}{$\begin{array}{ll}\text { DIFFERENCES } \\
\end{array}$} \\
\hline THEME/FACTOR & Ward A & Ward C \\
\hline \multicolumn{3}{|l|}{ Workload } \\
\hline Task focus & - & -- \\
\hline \multicolumn{3}{|l|}{$\begin{array}{l}\text { Leadership, Accountability and } \\
\text { Roles }\end{array}$} \\
\hline Leadership roles & - & -- \\
\hline \multicolumn{3}{|l|}{ Key } \\
\hline \\
\hline \multicolumn{3}{|c|}{$\begin{array}{l}\text { Discussed as a key theme from a negative perspective } \\
\text { Discussed as a minor theme from a negative perspective }\end{array}$} \\
\hline Discussed as a key the & e from a p & perspective \\
\hline Discussed as a minor $\mathrm{t}$ & me from a & ive perspective \\
\hline Discussed as a key the & e from bot & sitive and negative perspective \\
\hline Did not emerge as a th & me in this & \\
\hline
\end{tabular}

Table 39 demonstrates that there were several similarities between Medical ward A and Medical ward B in factors which participants believed affected care of acutely unwell ward patients on their wards. All similarities and differences in factors between the wards related to problems with workload, teamwork, communication and leadership, accountability and roles. 


\subsubsection{Workload}

Within the theme of workload having time and coping, nurse to patient ratio and skill mix were all identified by participants as important factors. Both wards were described by nursing participants as very busy due to the complexity of the patients who presented with multiple co-morbidities. Nursing participants from both medical wards described staff shortages and a poor skill mix, which contributed to their perception of an extremely heavy workload. Within Medical ward C this led to staff becoming task focused; however, this was not discussed as a key factor in Medical ward A. Both Medical ward A and Medical ward C had nursing vacancies. Neither ward collected data to demonstrate the acuity of their patient population. The rationale given by participants from both medical wards for not using an acuity tool was that the tool did not adequately capture the acuity of their patients.

\subsubsection{Teamwork}

Nursing participants from both medical wards believed that their wards were supportive environments in relation to caring for acutely unwell ward patients. Both wards had recently implemented a form of team nursing which was intended to increase the amount of support for staff and prevent staff from working in isolation. In both wards, nursing participants believed staff were willing to help each other; however they were often reticent to ask for help as they perceived that everyone was equally busy.

The level of clinical experience of both the nursing and medical staff was an important factor in both medical wards in terms of assessment skills and the ability to manage acutely unwell patients in a timely and appropriate manner. However, nursing participants described situations where their clinical experience was not acknowledged by medical staff, which led to frustration. Nursing participants stated that senior, more experienced, nurses had better assessment and communication skills, which meant that they captured the medical staff's attention more readily. 


\subsubsection{Communication}

Breakdown in communication was a common factor described by nursing and medical participants from both medical wards as affecting the care of acutely unwell ward patients. Nursing participants described scenarios where they struggled to get medical staff to review sick patients. Medical participants described their frustration at referrals from nursing staff which contained limited information, which impacted on their ability to determine the urgency of the request for review.

\subsubsection{Leadership, accountability and roles}

The main difference between the two medical wards concerned leadership roles, specifically the failure of the coordinator to provide shift leadership due to a perceived excessive workload. On Medical ward A the coordinator did not take a patient load (except for overnight), whereas the coordinator on Medical ward C always took a patient load as well as coordinating the ward, which nursing participants viewed as dangerous and as having an impact on how acutely unwell ward patients were cared for on that ward.

\subsection{Analysis of similarities and differences between Surgical ward B and Surgical ward D}

Using the findings from the cross-case analysis presented as Table 36, the key themes and factors were extracted and used to determine the similarities and differences between Surgical ward B and Surgical ward D. These findings are illustrated in Table 40.

Table 40. Similarities and Differences and between Surgical wards B and Surgical ward D

\begin{tabular}{|l|l|l|}
\hline \multicolumn{3}{|c|}{ SIMILARITIES } \\
\hline THEME/FACTOR & Ward B & \multicolumn{1}{l|}{ Ward D } \\
\hline Workload & \multicolumn{2}{|l|}{} \\
\hline Having time and coping & -- & -- \\
\hline Teamwork & & \\
\hline Relationships & ++ & + -- \\
\hline Support & ++ & ++ \\
\hline Clinical experience & -- & -- \\
\hline
\end{tabular}


Table $\mathbf{4 0}$ (continued). Similarities and Differences and between Surgical wards B and Surgical ward D

\begin{tabular}{|c|c|c|c|}
\hline \multicolumn{2}{|c|}{ THEME/FACTOR } & \multicolumn{2}{|l|}{ Ward B } \\
\hline \multicolumn{4}{|c|}{ Communication } \\
\hline \multicolumn{2}{|c|}{ Responding } & -- & -- \\
\hline \multicolumn{2}{|c|}{ Breakdown in communication } & -- & -- \\
\hline \multicolumn{4}{|c|}{$\begin{array}{l}\text { Leadership, Accountability and } \\
\text { Roles }\end{array}$} \\
\hline \multicolumn{2}{|c|}{ Leadership roles } & -- & -- \\
\hline \multicolumn{4}{|c|}{ DIFFERENCES } \\
\hline \multicolumn{2}{|c|}{ THEME/FACTOR } & Ward B & Ward D \\
\hline \multicolumn{2}{|c|}{ Workload } & & \\
\hline \multicolumn{2}{|c|}{ Nurse to patient ratio } & -- & - \\
\hline \multicolumn{2}{|c|}{ Allocation } & -- & ++ \\
\hline \multicolumn{4}{|c|}{ Teamwork } \\
\hline \multicolumn{2}{|c|}{ Credibility and Trust } & $\mathrm{x}$ & -- \\
\hline \multicolumn{2}{|c|}{$\begin{array}{l}\text { Organisational systems and } \\
\text { processes of care }\end{array}$} & & \\
\hline \multicolumn{2}{|c|}{ Service initiatives } & ++ & + \\
\hline \multicolumn{2}{|c|}{ Organisational policies } & -- & - \\
\hline \multicolumn{4}{|l|}{ Key } \\
\hline-- & \multicolumn{3}{|c|}{ Discussed as a key theme from a negative perspective } \\
\hline - & \multicolumn{3}{|c|}{ Discussed as a minor theme from a negative perspective } \\
\hline++ & \multicolumn{3}{|c|}{ Discussed as a key theme from a positive perspective } \\
\hline+ & \multicolumn{3}{|c|}{ Discussed as a minor theme from a positive perspective } \\
\hline \multirow{2}{*}{$\begin{array}{l}+/- \\
x\end{array}$} & \multicolumn{3}{|c|}{ Discussed as a key theme from both a positive and negative perspective } \\
\hline & \multicolumn{3}{|c|}{ Did not emerge as a theme in this case } \\
\hline
\end{tabular}

Table 40 demonstrates that participants from Surgical ward B and Surgical ward D believed that issues related to workload, teamwork, communication, and leadership, accountability, and roles, and organisational systems and processes of care affected care of acutely unwell patients.

\subsubsection{Workload}

Having time to care for all their allocated patients and coping with their workload was identified by nursing participants as stressful and at times distressing. Nursing participants described a ward environment where patient complexity was 
compounded by a rapid turnover of patient admissions and discharges. Having time to adequately care for all their patients was perceived by nursing participants as difficult most of the time. This difficulty in meeting all their patients' needs was exacerbated if a patient in their care became acutely unwell. In such circumstances nursing participants from both surgical wards believed that spending time with an acutely unwell ward patient was detrimental to the other patients in their care.

Nursing participants from both wards discussed allocation of patient loads as another issue affecting workloads, but from differing perspectives. On Surgical ward B patients were pre-allocated to nurses on the oncoming shift by the preceding coordinator. However nursing participants noted that changes in patient condition may occur over the course of the day, which could adversely affect the intensity of an allocated workload. On Surgical ward D patients were allocated at handover by the coordinator for that shift. This coordinator was more aware of the up to date information about each patient's condition. Furthermore, participants on Surgical ward D described allocation practices very positively and were comfortable requesting an allocation change.

On Surgical ward B nursing participants described staff shortages and an inadequate nurse patient ratio as an issue which at times impacted on their workload and the time they had to adequately care for acutely unwell ward patients. Being short staffed did not appear to be such an issue for Surgical ward D. Both surgical wards had an acuity measurement tool and all staff were expected to input acuity data each shift. However, Surgical ward D had strategically used acuity data to secure additional FTEs, which may be why nurse patient ratios was not such an issue for this ward.

\subsubsection{Teamwork}

Within the theme of Teamwork the focus on relationships on both Surgical ward B and Surgical ward D was very apparent. Both wards had staff who had worked on the wards for a long time. Both surgical wards had managed to retain senior staff to provide a backbone of experienced nurses. This may have been due to the fact that both CNMs believed in the importance of socialising as a group (both medical and nursing staff) outside of working hours as a way of strengthening 
working relationships. This investment in staff as people rather than just colleagues appeared to be beneficial in the area of staff retention.

Nursing and medical participants from both surgical wards commented on the strength of the relationships which developed between nursing staff and surgical registrars, which facilitated good teamwork. Nursing participants commented that the registrars' rotations on the ward were longer, allowing them to get to know each other better, which led to a mutual appreciation of each other's skills and experience.

In contrast, nursing participants from Surgical ward D described difficulties associated with hierarchical relationships between nursing and some consultant medical staff which was not apparent in Surgical ward B. A change in surgical leadership had occurred at Hospital 2 which may account for this difference. Under the previous leadership, nursing staff had been invited to attend particular surgical meetings; a practice which had not continued. As such the CNM felt that nursing staff within the ward had no formal voice.

Again within the theme of teamwork, both wards were described by participants as supportive areas to work in. Overall, nursing participants described both wards as having a culture where asking for help was acceptable, although some nursing participants believed junior nursing staff were often hesitant to ask because of the perceived workload pressures of their colleagues. Not asking for help was viewed by some senior nursing and medical participants as an issue which affected the care of acutely unwell ward patients. They believed that junior nursing and medical staff tried to cope at times when they clearly needed additional support from more experienced staff.

Levels of clinical experience were also important in relation to teamwork. Having a core of senior nurses who could provide advice and expertise was important for supporting junior staff caring for acutely unwell ward patients. In addition, both nursing and medical participants believed senior nurses and doctors appeared to have better assessment skills and were able to act more quickly when managing acutely unwell patients. 


\subsubsection{Communication}

Breakdown in communication was an issue in both surgical wards. Despite both surgical areas using the SBAR communication framework, medical participants were still concerned that they did not receive adequate information when nurses were referring patients for urgent review. Nursing participants also believed that at times their requests for review were not responded to appropriately.

A possible explanation for this is due to credibility and trust. Nursing participants on Surgical ward D believed that more senior nurses got action quicker because medical staff believed senior staff to be more clinically credible and trusted their judgement more. However, this attitude frustrated the senior nursing participants on Surgical ward D as they believed that these credibility judgements left some more junior staff feeling undervalued. This was not discussed as a key factor on Surgical ward B and would support the concept of some dysfunctional hierarchical relationships described by nursing participants in Surgical ward D.

\subsubsection{Organisational systems and processes of care}

The main difference between the two surgical wards was associated with the organisational systems and processes of care. Participants from Surgical ward B were very positive about service initiatives such as CCOS and EWS and the support and direction these initiatives provided in the care of acutely unwell ward patients. At the time of the study in Hospital 2 no such service initiatives existed, although After Hours Support Nurses (AHSN) did provide additional clinical support after hours. However the support these nurses provided was not discussed as a key factor in the care of acutely unwell ward patients in Surgical ward D.

A second difference between the two surgical wards was the issue of caring for medical outliers. Surgical ward B received many medical outliers whereas Surgical ward D did not. Surgical ward D covered multiple surgical specialties and was the main receiving ward for patients from the surgical high dependency unit (HDU). As such Surgical ward D rarely had sufficient beds to take any admissions that were not surgical. Nursing participants on Surgical ward D would not have encountered the same difficulties that nursing participants on Surgical ward B did in 
the care and management of this group of patients therefore this may account for why this was not discussed as an issue in this ward.

\subsection{Analysis of similarities and differences in factors between medical and surgical wards in Hospital 1 and Hospital 2}

Using the findings from the cross-case analysis presented as Table 36, the key themes and factors were extracted and used to determine the similarities and differences between the medical and surgical wards across both organisations. These findings are illustrated in Table 41.

Table 41. Similarities and differences in factors between medical and surgical wards in Hospital 1 and Hospital 2

\begin{tabular}{|c|c|c|c|c|}
\hline \multicolumn{5}{|c|}{ SIMILARITIES } \\
\hline THEME/FACTOR & $\begin{array}{l}\text { Ward } \\
\text { A }\end{array}$ & $\begin{array}{l}\text { Ward } \\
\text { B }\end{array}$ & $\begin{array}{l}\text { Ward } \\
\text { C }\end{array}$ & $\begin{array}{l}\text { Ward } \\
\text { D }\end{array}$ \\
\hline \multicolumn{5}{|l|}{ Workload } \\
\hline Having time and coping & -- & -- & -- & -- \\
\hline \multicolumn{5}{|l|}{ Teamwork } \\
\hline Support & $+/-$ & ++ & ++ & ++ \\
\hline Clinical experience & -- & -- & -- & -- \\
\hline \multicolumn{5}{|l|}{ Communication } \\
\hline Responding & -- & -- & -- & -- \\
\hline Breakdown in communication & -- & -- & -- & -- \\
\hline \multicolumn{5}{|c|}{ DIFFERENCES } \\
\hline THEME/FACTOR & $\begin{array}{l}\text { Ward } \\
\text { A }\end{array}$ & $\begin{array}{l}\text { Ward } \\
\text { B }\end{array}$ & $\begin{array}{l}\text { Ward } \\
\text { C }\end{array}$ & $\begin{array}{l}\text { Ward } \\
\text { D }\end{array}$ \\
\hline \multicolumn{5}{|l|}{ Workload } \\
\hline Task Focus & - & - & -- & - \\
\hline Allocation & - & - & $x$ & ++ \\
\hline Nurse to patient ratio & -- & -- & -- & - \\
\hline Skill mix & -- & - & -- & - \\
\hline \multicolumn{5}{|l|}{ Teamwork } \\
\hline Relationships & - & ++ & - & $+/-$ \\
\hline Credibility and Trust & - & $x$ & - & -- \\
\hline \multicolumn{5}{|c|}{ Leadership, Accountability and Roles } \\
\hline Leadership roles & - & -- & -- & -- \\
\hline \multicolumn{5}{|c|}{$\begin{array}{l}\text { Organisational systems and processes of } \\
\text { care }\end{array}$} \\
\hline Service initiatives & + & ++ & - & + \\
\hline Organisational policies & - & -- & - & - \\
\hline
\end{tabular}




$\begin{array}{ll}-- & \text { Discussed as a key theme from a negative perspective } \\ - & \text { Discussed as a minor theme from a negative perspective } \\ ++ & \text { Discussed as a key theme from a positive perspective } \\ + & \text { Discussed as a minor theme from a positive perspective } \\ +/- & \text { Discussed as a key theme from both a positive and negative perspective } \\ X & \text { Did not emerge as a theme in this case }\end{array}$

Table 41 demonstrates that the main themes to emerge from the cross-case analysis were workload, teamwork, communication, leadership, accountability, and roles, and organisational systems and processes of care.

\subsubsection{Workload}

Irrespective of which ward and hospital participants worked in, workload was an issue. Nurses felt swamped by the number and type of patients they were expected to care for on any given shift and this became worse when shifts were short staffed or after hours. Medical participants described large volumes of patients to care for when on call with little senior support. Nursing participants from all four wards described being short staffed, leading to a poor nurse patient ratio and insufficient time to care for patients.

Skill mix was an issue in both medical wards but not in either of the surgical wards. In both surgical wards there was a core of senior nurses who had worked on the ward for a long time. This was not the case on the medical wards. This may have been due to the emphasis that the surgical CNMs placed on developing relationships within their wards and the effort that was put into staff socialising, which helped retain skilled staff.

The only ward where nursing participants did not mention nurse patient ratio as a factor affecting care of acutely unwell ward patients was Surgical ward D. This ward had used patient acuity data to make a case for additional staff. Furthermore, the CNM on this ward was very flexible about employment contracts, which allowed staff to continue to work on the ward when their personal circumstances changed e.g. after having children. This also may have contributed to the retention of staff. 


\subsubsection{Teamwork}

Teamwork was a key theme across all four wards. All nursing participants described a supportive ward culture where staff were willing to help each other. However not having enough staff with sufficient clinical experience negatively affected the amount of support available. Participants believed that experienced healthcare staff could assess and manage acutely unwell patients more effectively. When nurses' clinical experience was not recognised or appreciated by medical staff, team working was affected.

Nursing participants from the two surgical wards described relationships as a key factor which affected teamwork. Investment in relationships was more apparent within the surgical wards, with both nursing and medical staff being encouraged to get to know each other socially to augment working relationships. However, hierarchical attitudes between doctors and nurses were perceived to be detrimental to working as a team within Surgical ward D.

\subsubsection{Communication}

Breakdown in communication and responding to requests for patient review were seen to be factors irrespective of the ward or hospital and also irrespective of any initiatives which had been introduced to try and facilitate better communication. From a nursing perspective, the ability to elicit a response from the medical team was the main factor. From the doctors' perspective, the main issue was the quality and timeliness of the information given by the nursing staff. The purpose of all communication initiatives that both organisations had implemented was to meet these communication shortfalls; so both organisations had some understanding of where the communication shortfalls lay. However, participants across both hospitals still identified poor communication and getting a response from medical staff as key factors. This suggests that implementation of the communication initiatives was not completely successful. Further, lack of evaluation of these initiatives meant that any problems had not been highlighted. 


\subsubsection{Leadership, accountability and roles}

In three out of the four wards nursing participants believed that leadership roles were a factor, specifically the excessive responsibilities and workload of the coordinator. Nursing participants believed that the coordinator's responsibilities were excessive because they were required to take a full patient load. This, participants believed, affected the coordinator's ability to have oversight for the ward. Not having this oversight meant that acutely unwell ward patients may not be recognised, particularly if being cared for by junior staff. The only ward not to highlight coordination as an issue did not have a requirement for its coordinator to take a patient load as well as coordinate the ward.

Coordination of a shift is clearly a pivotal role, yet there was no specific training, no clear expectations (other than a job list and a resource folder) for the role on any of the wards. Furthermore, on each of the wards the selection of coordinator was made on the basis of seniority and experience, which was dependent upon whether there were sufficient numbers of senior staff to fulfil that role, and how each ward defined seniority. Under ideal circumstances, when wards were fully staffed, the selection of the coordinator was not an issue. However, when there were no senior staff available, the role of the coordinator could be assigned to someone designated as intermediate in terms of skills and experience, which nursing participants described as potentially dangerous.

\subsubsection{Organisational systems and processes of care}

Nursing participants from Surgical ward B highlighted the support from new service initiatives such as CCOS and EWS as a factor which affected the care of acutely unwell ward patients. Despite being the main user of this service within Hospital 1, participants from Medical ward A did not identify the support these initiatives provided as a key issue. No such service initiatives existed in Hospital 2, which is why these initiatives do not feature as supportive factors in Hospital 2. However, Hospital 2 had implemented other service initiatives such as 'The Productive ward: Releasing time to care' which was being rolled out on Surgical ward D. As the name states, this initiative is designed to empower nursing staff to 
organise their ward more efficiently in order to make more time available to care for patients. However this was not identified by nursing participants as a means of providing support in caring for acutely unwell patients.

\subsection{Cross-hospital analysis}

In order to demonstrate the similarities and differences between organisations, a cross-hospital analysis was carried out. Both hospitals were analysed individually. Transcripts of participants who were not specifically attached to any of the wards (cases) but worked across the organisation (e.g. EMTH2, SenManSPH1) were coded separately as either Hospital 1 or Hospital 2 using the analytical framework (see Chapter 5). Key themes and factors were then extracted. These key themes and factors were mapped against the analytical framework to form a cross-hospital matrix similar to that used for the cross-case analysis. The similarities and differences were then extracted to formulate Table 42 . Table 42 demonstrates the similarities and difference between Hospital 1 and Hospital 2.

Table 42. Cross-hospital analysis - similarities and differences

\begin{tabular}{|l|l|l|}
\hline \multicolumn{3}{|c|}{ SIMILARITIES } \\
\hline THEME/FACTOR & Hospital 1 & Hospital 2 \\
\hline Teamwork & + -- & -- \\
\hline Relationships & & \\
\hline Communication & -- & -- \\
\hline Responding & -- & -- \\
\hline Breakdown in communication & & \\
\hline $\begin{array}{l}\text { Organisational systems and } \\
\text { processes of care }\end{array}$ & ++ & $+/-$ \\
\hline Service initiatives & -- & -- \\
\hline Organisational policies & \multicolumn{2}{|l|}{} \\
\hline Culture and Environment & $+/-$ & + - \\
\hline Organisational culture & \multicolumn{2}{|l|}{} \\
\hline Skills and Knowledge deficit & -- & -- \\
\hline Workforce preparation & DIFFERENCES \\
\hline \multicolumn{2}{|l|}{} \\
\hline THEME/FACTOR & \multicolumn{2}{|l|}{} \\
\hline Workload & - & + Hospital 2 \\
\hline Having time and coping &
\end{tabular}


Table 42 (continued). Cross-hospital analysis - similarities and differences

\begin{tabular}{|c|c|c|}
\hline \multicolumn{3}{|c|}{ DIFFERENCES } \\
\hline THEME/FACTOR & Hospital 1 & Hospital 2 \\
\hline \multicolumn{3}{|l|}{ Teamwork } \\
\hline Skill mix & $x$ & $+/-$ \\
\hline Support & - & -- \\
\hline \multicolumn{3}{|c|}{$\begin{array}{l}\text { Leadership, Accountability and } \\
\text { Roles }\end{array}$} \\
\hline Leadership roles & -- & - \\
\hline \multicolumn{3}{|c|}{ Organisation of $24 / 7$ services } \\
\hline Support & -- & - \\
\hline Continuity of care & -- & - \\
\hline \multicolumn{3}{|c|}{ Culture and Environment } \\
\hline Ward culture & $+/-$ & - \\
\hline \multicolumn{3}{|c|}{ Skills and Knowledge deficit } \\
\hline Assessment skills & - & -- \\
\hline
\end{tabular}

\section{Key}

$\begin{array}{ll}-- & \text { Discussed as a key theme from a negative perspective } \\ - & \text { Discussed as a minor theme from a negative perspective } \\ ++ & \text { Discussed as a key theme from a positive perspective } \\ + & \text { Discussed as a minor theme from a positive perspective } \\ +/- & \text { Discussed as a key theme from both a positive and negative perspective } \\ X & \text { Did not emerge as a theme in this case }\end{array}$

Table 42 shows the following key themes that emerged from the cross-hospital analysis: workload; teamwork; communication; leadership, accountability, and roles; organisational systems and processes of care; organisation of 24/7 services; culture and environment; and skills and knowledge deficit. As with all previous analyses, the key themes are discussed in order of the weight of the data.

\subsubsection{Organisational systems and processes of care}

Executive management participants from both hospitals recognised that the care of acutely unwell ward patients had been challenging in the past, which had led to serious and sentinel events. These participants believed they were aware of the clinical risks patients faced and had developed an organisational culture which made patient safety a priority. Both hospitals had implemented service initiatives which they believed were supportive to healthcare professionals when caring for 
acutely unwell ward patients. However, according to some senior management and nursing participants their organisation did not appear to have clear strategies for implementation and evaluation of the initiatives. Both hospitals had made service initiative changes in reaction to the occurrence of serious or sentinel events. Hospital 2 appeared to have a more developed strategy for improving patient safety, as demonstrated by the documentation reviewed (see Table 28); however, several changes were being implemented at one time and this, coupled with a complete nursing and midwifery leadership restructure, appeared to stall some aspects of the patient safety strategy.

Hospital 2 also had services which could assist healthcare staff care for acutely unwell ward patients that participants perceived were underutilised. For example, the AHSNs appeared frustrated at not being used sufficiently by the ward staff and not being valued by the organisation. The organisation was making plans to implement some form of CCOS, when this team of nurses were already part of the organisation and were in a role which they believed offered a similar type of service. The underuse of these nurses may have been due to a lack of understanding of what the service offered or was capable of. Again this may be due to implementation and evaluation of service initiatives within the hospitals.

\subsubsection{Teamwork}

Teamwork was seen as key to the provision of good care for acutely unwell ward patients in both hospitals. Executive and senior management participants from both hospitals believed that focussing on developing good relationships between team members would enhance good team working. Where the two hospitals differed was that participants from Hospital 1 believed that relationships were on the whole good; participants from Hospital 2 highlighted issues related to poor behaviours and attitudes which participants believed affected care of acutely unwell ward patients.

\subsubsection{Communication}

Breakdown in communication was recognised as an issue across both hospitals. Participants in all roles described problems in both handovers between 
nurses, and handovers between doctors, as well as problems with communication between doctors and nurses. Both hospitals had implemented new forms of communication frameworks such as SBAR in an attempt to alleviate communication problems between healthcare professionals.

\subsubsection{Skills and knowledge deficit}

The theme of skills and knowledge deficit emerged as a consistent theme across both hospitals. Executive and senior management participants from both hospitals queried whether undergraduate education prepared the medical and nursing workforce adequately. There appeared to be a desire to return to the old way of training and a view that the changes in undergraduate education which had occurred were not necessarily for the better. In Hospital 2, inadequate assessment skills were of particular concern to executive and senior management participants, whereas in Hospital 1 this was not a key factor. This may be due to either the backto-basics campaign or EWS implemented at Hospital 1.

\subsubsection{Culture and environment}

The main similarity within this theme is that executive management participants from both hospitals believed that their organisation promoted and was committed to a strong patient safety culture. Management participants from Hospital 1 believed the culture of the ward affected how acutely unwell ward patients were cared for. These participants commented on the differences between the medical and surgical wards in Hospital 1 in terms of culture. Historically the culture on Medical ward A had been highlighted as an issue. The relatively recent sentinel event on the medical ward and subsequent raft of care delivery changes may account for the medical ward being uppermost in participants' minds and why this was not a theme to emerge from both hospitals.

\subsubsection{Leadership, accountability and roles}

In Hospital 1 there had been a focus on leadership roles specifically related to accountabilities and responsibilities at ward leadership level. However this focus had not been directed at the level of the coordinator, a role which was highlighted 
by some senior management participants as requiring review. In their view, this role was difficult, as the coordinator had to take a full patient load and did not have sufficient time for oversight of the rest of the ward. Furthermore these participants felt there was insufficient investment in preparing nursing staff to take on this role in terms of training and providing support and direction.

In Hospital 2 leadership roles did not emerge as a key theme despite the Hospital being in the throes of a nursing leadership restructure at the time of this study. Again, this restructure aimed to clarify responsibilities and accountabilities of nursing management.

\subsubsection{Organisation of $24 / 7$ services}

Executive and senior management participants from Hospital 1 recognised that there were some serious concerns in the provision of care for patients and support for staff after hours, and at the time of the study the organisation was working towards a solution. From an organisational perspective the provision of after hours care had been highlighted due to a HDC case demonstrating the reactionary nature of some of this organisation's decision-making regarding the care of acutely unwell ward patients.

\subsubsection{Workload}

Executive participants from Hospital 2 felt strongly that there were sufficient numbers of experienced staff to provide care for acutely unwell ward patients. This was not a view supported by other senior managers or senior nurses who worked across the hospital.

\subsection{Similarities and differences between wards and organisational viewpoint regarding factors affecting care of acutely unwell ward patients}

There are similarities and differences between the key themes which emerged from the data during the cross-case and cross-hospital analyses. The main findings from the cross-case analysis demonstrate that according to ward-based participants, factors affecting care of acutely unwell ward patients are related to workload, teamwork, communication, and leadership, accountability, and roles. 
In contrast, findings from the cross-hospital analysis demonstrate that participants who worked across the organisation (i.e. not on one of the wards designated as cases in this study) viewed issues related to organisational systems and processes of care, culture and environment, teamwork, communication, and skills and knowledge deficit, as key factors.

The cross-case and cross-hospital analyses reveal that participants at ward and organisational level agreed that teamwork and communication were factors affecting care of acutely unwell ward patients. However, there was also a difference in perspective regarding these factors between participants within organisations, dependent upon their role in the organisation.

Executive management, senior management, and ward-based participants viewed factors (workload, teamwork) from different perspectives. Within both organisations there appeared to be a 'them and us' divide - a disconnection not only in perceptions of factors affecting care of acutely unwell ward patients but also what was required to make caring for acutely unwell patients more successful. For example, on Medical ward A several roles had been developed to assist nursing staff in specific aspects of care for patients such as the complex discharge coordinator. This role was cited by senior management as a support role for nursing staff which was intended to alleviate the workload of ward nursing staff. However, this sort of support did not appear to register as tangible additional support by ward nursing staff. What ward nurses wanted were extra nurses on the shifts to increase the nurse patient ratio. These specific roles were not perceived as part of the nursing numbers and therefore were not available to alleviate the workload burden.

In addition, some executive and senior management participants at both organisations appeared to have the viewpoint that nursing staff were not doing enough to help themselves to make their workload better. This is particularly apparent in Hospital 2, where executive management participants described the 'tyranny of busyness' that nurses had talked themselves into. This is in contrast to the view of senior management in Hospital 2, who believed that executive management were out of touch with the realities of working on the ward. 
In Hospital 1 some senior management participants demonstrated a similar attitude to executive management participants at Hospital 2. These participants suggested that nursing staff wanted everything 'handed to them on a plate' and had little understanding of the fiscal restraints that senior management worked under. The reality for senior management participants was that the only way to make any changes designed to improve care of acutely unwell ward patients was to divert existing resources, and therefore they encouraged nursing staff to 'just get on with it'.

\subsection{Summary}

As a result of the synthesis of the within-case, cross-case, and cross-hospital analyses, four key findings have emerged from this study to answer the research question.

1. Issues related to the themes of workload, teamwork and communication were key factors affecting care of acutely unwell ward patients in the four wards studied in this research. The ability of the coordinator to provide shift leadership was a significant factor for three of the four wards.

2. Teamwork and communication are the only two themes in common between ward-based participants and participants who worked across the organisations. Participants from both surgical wards believed that focusing on relationships was important to good team working. This may be associated with their ability to retain staff and therefore to have a core of senior staff.

3. Participants who worked across the organisations believed that issues related to organisational systems and processes of care, culture and environment, and skills and knowledge deficit were key factors affecting the care of acutely unwell ward patients.

4. There is a difference in perspective regarding factors affecting care of acutely unwell ward patients depending upon the participant's role within the organisation. 
These findings have significant implications for health care staff. In Chapter 7 the key findings from this study will be discussed with reference to the theoretical framework presented in Chapter 2. This will demonstrate how these findings not only support, but add to, current theory regarding the suboptimal care of the acutely unwell ward patient. 


\section{CHAPTER 7: DISCUSSION}

\subsection{Introduction}

The multilevel analysis has produced four key findings which have significant implications for wards and organisations. This chapter will demonstrate how these key findings support previous research and add new insights to the body of knowledge about factors affecting care of acutely unwell ward patients. This chapter begins with a summary of the research, reviewing the aims, objectives, and methods used to conduct this multiple case study. A discussion of the findings follows which positions this study in the context of what is currently known about the factors affecting care of acutely unwell ward patients, determined through the concept analysis which has formed the theoretical framework underpinning this research. Through this the contribution of this study to understanding on factors affecting acutely unwell ward patients is made explicit.

\subsection{Summary of research design}

Drawing on the experiences of ward nurses, doctors, and hospital executive and senior managers, the aim of the study was to make clear the main factors which impede or assist care delivery of acutely unwell ward patients on a general surgical or medical ward. Two research objectives also guided this research. Firstly to determine if factors affecting care of acutely unwell ward patients were unique to the ward or the hospital the ward was in, or whether common factors existed. Secondly, to determine whether multiple perspectives existed within an organisation regarding factors affecting acutely unwell ward patients. A multiple case study methodology was used to study four wards (two surgical and two medical) in two North Island hospitals in New Zealand. Data were collected via interviews, focus groups, and documentation review. Data were analysed using NVivo 8 software using the techniques of categorical aggregation and pattern matching. 


\subsection{Key findings of this study}

This study has demonstrated from the perspective of nurses, doctors, and managers that factors affecting care of acutely unwell ward patients are multifaceted and interrelated. This study has also identified that the majority of factors affecting care of acutely unwell ward patients are not unique to the individual cases but common factors are present across all four cases and both organisations. However, how these factors are perceived differ across organisational roles. A key finding from this study was how ward-based staff perceived workload as affecting care of acutely unwell ward patients. Doctors and nurses identified that excessive workloads caused by increased patient acuity and staff shortages impacted on time to care for acutely unwell ward patients. A further finding from this study was that participants across all four cases, and both hospitals, and across all organisational roles, identified that teamwork and communication were important factors affecting care of acutely unwell patients. Finally, organisational systems and processes, culture and environment, and skills and knowledge deficit were also influential in impacting on care of acutely unwell ward patients. What was evident in some of the factors was that ward based and executive management staff held different, and at times polarised, perspectives.

\subsubsection{Workload}

This study demonstrates that nurses and doctors perceived that an increased workload affects their ability to adequately care for acutely unwell ward patients. In this study, ward-based participants reported that workload was affected by inadequate nurse patient ratios, poor skill mix, and unsafe patient allocation. Nursing and medical participants from all four cases described their working environment as very busy, leaving them with little time to adequately care for patients. This supports findings from UK-based research which demonstrates that workload does affect nurses' and doctors' ability to care for acutely unwell ward patients (Chellel, Higgs, \& Scholes, 2006; Cox, James, \& Hunt, 2006; Cutler, 2002; NPSA, 2007; Wood, Douglas \& Priest 2004). This study adds to the body of New Zealand-based health knowledge in concurring with Lawless, Wan, \& Zeng (2010), 
whose survey of NZ nurses indicated that nurses believed that increased workload led to an unsatisfactory standard of care being delivered.

Increased workload resulted in nurses having to make difficult choices between attending to acutely unwell ward patients or to the needs of the other patients. These difficult choices provoked strong emotions in nursing participants who used words such as "guilt" to describe their feelings about some of the choices they felt compelled to make due to heavy workloads. As a consequence of choosing to care for acutely unwell patients, nurses felt they were neglecting other patients in their care. This dilemma concurs with findings from Cutler, (2002) Cox, James, and Hunt (2006) and Lawless et al., (2010). Nursing participants in this study described two strategies to ameliorate these feelings of neglect.

Firstly, nursing participants described missing meal breaks and staying on beyond the end of their shift to complete their work. This study supports Lawless et al.'s (2010) survey that demonstrated that $79.5 \%$ of nurses frequently worked past the end of their shift to complete work or cover staffing shortfalls. In this study and Lawless et al.'s survey, working beyond end of shift was an organisational norm. This 'overtime' did not universally result in an overtime claim and was perceived by nurses to be a professional obligation to meet patients' needs.

Secondly, nurses adopted an approach that has been described by Lawless et al. (2010) as care rationing, "where any aspect of patient care is either unduly delayed, unable to be completed to a satisfactory level or omitted due to workload pressures" (p. 17). In this study, in order to manage the choices to be made in managing workload, nurses became task focused and were reluctant to increase the frequency of observations on patients. This is of concern, as lack of such surveillance may result in suboptimal care of acutely unwell patients (Clarke \& Aiken, 2003).

In this study a key factor that influenced the need to ration care was the presence of staff shortages, as demonstrated by the data from the within case analysis (Surgical ward B and Medical ward C). Reduced workforce in conjunction with heavy workloads was considered by nursing and ward management participants as important factors affecting care of acutely unwell ward patients. 
The resultant reduction in nurse patient ratios is of concern, as there is a well established international body of evidence demonstrating the substantial impact of reduced nurse staffing ratios on patient mortality (Aiken et al., 2002; Tourangeau et al., 2006; Rafferty et al., 2007).

Despite this convincing body of research supporting the need for adequate nurse patient ratios there is no evidence to suggest that organisations are moving towards a fundamental review and increase of ward establishments. Instead, healthcare organisations are attempting to ameliorate this clinical risk through implementation of service delivery change to support and educate nurses and junior medical staff in the care of acutely unwell patients. Across the case studies reported here this has included such service delivery changes as critical care outreach services (CCOS), early warning scores (EWS), The Productive Ward, SBAR, colour coded observation charts and ALERT training courses. Additional nursing roles such as complex care coordinators, patient flow coordinators, complex discharge nurses, and pre-assessment nurses have also been developed with the intention of alleviating some of the ward nurse's workload.

Whilst the literature and participants in this study agree that these service changes have been well received by nursing and medical staff, this study highlights that such initiatives are not recognised by nursing staff as alleviating the workload burden. The introduction of new nursing roles and organisational systems and processes is not perceived by nursing staff as attending to the "latent conditions which predispose individuals to make mistakes" (Clarke \& Aiken, 2006, p. 2). This view is in contrast to some executive managers in Hospital 2 who described nurses talking themselves into a 'tyranny of busyness'. This resulted in executive managers appearing to demonstrate little empathy or understanding for staff members' perceptions of their workload. In addition some senior management participants (from Hospital 2) perceived that executive management lacked an understanding of the contemporary clinical pressures faced by ward based staff. This supports the view that organisational thinking is "too ethereal and distant for the day to day concerns of clinicians and patients to be of much use" (Clarke \& Aiken, 2006, p. 2). 
Attending to the root cause of suboptimal care requires organisational commitment to a shared governance model where all staff from ward to executive level are able to inform organisational decision making. The NPSA (2007) suggests that this could be achieved through a Deteriorating Patient Group. They suggest a model where the group has multi disciplinary membership and links "with local structures and processes, including links with clinical governance and risk management, workforce planning, education programmes, critical care staff and strategic health authorities/regional offices" (NPSA, 2007, p. 27).

It is clear that workload is a complex contemporary issue that must be understood in the context in which it occurs. In this study, all participants identified workload to be a key factor affecting care of the acutely unwell ward patient. However, whilst executive management teams supported new organisational processes and service delivery changes to address suboptimal care, ward-based staff identified a more fundamental workforce concern. It is suggested that until a model of engagement that enables agreement about workload factors occurs, then suboptimal care will continue to be present in surgical and medical wards.

\subsubsection{Teamwork}

This study demonstrates that participants across all levels of the organisation perceive effective teamwork to be an important factor affecting the care of acutely unwell ward patients. In this study teamwork was shown to be affected by the availability of senior, experienced staff to provide support for team members and relationships between team members. This finding supports and contributes to findings from UK- and Australian-based studies, where the importance of teamwork when caring for acutely unwell ward patients has been clearly demonstrated (Endacott, et al., 2007; NCEPOD, 2005; NPSA, 2007). These studies highlight factors such as shift patterns, staff shortages, and reliance on locum and agency staff as negatively impacting on teamwork. Whilst supporting the findings of this earlier research, this study adds to what is already known about how teamwork affects the care of acutely unwell ward patients by providing some additional insights. This includes the importance that participants placed on support as a key aspect of teamwork when caring for an acutely unwell ward patient. In this study, ward- 
based participants described two different sources of support; support from within the ward-based team and, support from outside the ward-based team.

\subsubsection{Support from within the ward-based team}

Support from within the ward-based team was reliant upon the availability of experienced team members. In this study nursing participants described how having sufficient numbers of experienced nurses rostered per shift provided a supportive culture in caring for acutely unwell patients. Nursing participants described experienced nurses as providing guidance and expertise in caring for acutely unwell ward patients as well as being able to communicate with medical staff more effectively. This finding concurs with research which has demonstrated that senior nurses with high levels of clinical experience are more assertive, and have greater confidence when communicating with medical staff (Andrews \& Waterman, 2005; Cox et al., 2006; NPSA, 2007).

Junior doctors who participated in this study also raised concerns about the lack of senior medical support they received, particularly after hours. Findings from this study support research which demonstrates that the most junior doctors are reviewing the sickest patients with minimal support (Hodgetts et al., 2002; NCEPOD, 2005; NPSA, 2007). To support junior doctors in their care of acutely unwell ward patients a raft of strategies has been suggested. These include calling in senior doctors when patients or work volumes are difficult, increased hands on involvement of consultants in acute care, and closer supervision of junior doctors (McQuillan et al., 1998; NCEPOD, 2005).

However, whilst appreciating the value of senior support from within the ward-based team, nurses, and doctors in this study expressed a reluctance to ask for support, fearing either they were adding to their colleagues' workloads or fearing admonishment. This reluctance to seek help is understandable when considering previous comments from nursing staff about workload issues, but it may also be due to difficulties establishing and maintaining relationships between ward-based team members.

In this study participants identified that team working was affected by insufficient time to develop relationships; lack of acknowledgement by medical staff 
of nurses' clinical abilities; and hierarchical relationships between doctors and between nurses and doctors. Nursing participants suggested that the short length (three months) of junior doctors' rotations through medical and surgical wards made it difficult to develop relationships, whilst one year rotations (as with registrars) allowed for much better relationship development. This supports research identifying time as critical to the development of interprofessional trust (NPSA, 2007).

In exploring these issues further, nurses did not feel their clinical judgements were trusted by medical staff. This lack of acknowledgement by doctors of nurses clinical abilities was detrimental to effective team working. Nursing staff avoided contacting medical staff they believed did not value or trust their clinical experience. In the view of nursing participants, time taken to seek a more approachable member of the medical team caused delays in patients receiving appropriate management.

Due to the excessive workloads described earlier and the shorter medical rotations, it is possible that there was limited time available to develop trusting work relationships. It could be suggested also that the lack of time to develop these relationships may perpetuate the existence of traditional hierarchical relationships based on role and professional discipline. In this study the negative impact of hierarchical relationships between nurses and doctors and, junior and senior doctors described by participants (particularly in Hospital 2), supports research which has demonstrated that communication is detrimentally affected where professional hierarchies exist (NPSA, 2007; Endacott et al., 2007).

To add to this important finding, nursing participants in this study identified developing social relationships (through outside work events) as key to effective teamwork and as possibly affecting retention of senior nurses as evidenced by staffing data from Surgical wards B and D. Whilst the suboptimal care literature acknowledges the importance of teamwork when caring for acutely unwell ward patients, this finding adds new insights for future strategy development aimed to enhance team working. 
Finally, in this study there was organisational recognition at executive and senior management level that strategies to improve teamwork were required. This was demonstrated by a change in the model of care from individual patient allocation to team nursing in both hospitals. However, in both organisations staff shortages and skill mix issues had impacted on the effectiveness of this new model of care. This supports the contention that inadequate staffing levels will negatively impact on care and hinder new ways of working such as team nursing (Derham, 2007). Ironically in this study, in one ward team nursing was specifically introduced to improve support for junior nursing staff. However the initiative was hampered by the lack of senior nursing staff to provide that support.

\subsubsection{Outside of the ward-based team}

This study shows that when support was not available from within the wardbased team or when specialty expertise was required, participants sought support and advice from outside of the ward. This was done through accessing support services such as CCOS and through patient referral to another specialty. This finding is corroborated by studies describing the value of critical care outreach services (CCOS) in supporting ward nurses in the care of acutely unwell ward patients (Chellel, Higgs \& Scholes, 2006; Pittard, 2003). However findings from this study also highlight the importance of availability and accessibility of such expertise. In this study Hospital 1 provided CCOS, but this was available during afternoon shifts only. Furthermore, participants from both hospitals outlined the need for clearer, more rapid referral policies to ensure timely assistance in caring for acutely unwell ward patients.

This study has shown that the provision of support both within and outside of the ward-based team is a key factor in the care of acutely unwell ward patients. Developing organisational strategies, including increasing the duration of junior doctors' rotations and encouraging social relationships between ward-based staff to promote the development of teamwork, are recommended. Reviewing policies which determine the availability and accessibility of resources is essential to the safe care of acutely unwell ward patients. 


\subsubsection{Communication}

All nurses, doctors, and managers in this study perceived communication issues to be a major factor affecting the care of acutely unwell ward patients. Both nursing and medical participants described occasions where poor communication affected care of unwell patients. Nursing participants reported difficulties getting medical staff to respond to requests for urgent review. Senior nursing participants recognised that communication style was critical to getting the appropriate response from medical staff. This was evidenced by how senior and outreach nurses were able to get the attention of medical staff more quickly, due to their confidence and expertise. Medical participants were frustrated by nurses' failure to provide sufficient information when referring a patient for urgent review. Clear and detailed information was required by medical participants for them to triage patients in regards to urgency.

These findings concur with communication difficulties described in the suboptimal care literature. The importance of packaging communication effectively using convincing referral language, medical terminology, and quantifiable vital signs data has been well described (Andrews \& Waterman, 2005; Chellel, Higgs \& Scholes, 2006).

Both organisations in this study cited how communication problems had been addressed by implementing the SBAR communication framework. Communication frameworks such as SBAR have been implemented worldwide in an attempt to provide a better structure for communication between team members. However the SBAR framework only provides a template for the type of information required The content of the information (detail, quantifiable data, language used) in each section is not specified and the manner in which this information is communicated (assertive, confident) is not necessarily obvious to those who use it.

The findings from this study add to the current body of knowledge by demonstrating that despite having communication frameworks in place, communication difficulties still exist. It is suggested that ongoing education and training is required to ensure that nursing staff are clear about what information is required and how to communicate it. 


\subsubsection{The role of the coordinator}

In this study coordinators were unable to provide effective shift leadership when taking a patient load. This was seen by nurses as a major factor affecting the care of acutely unwell ward patients. Two key issues were emphasised by participants: lack of preparation for the role and excessive responsibilities.

Nurses who undertook the role of coordinator did not receive any formal training or preparation to carry out the role. This finding supports research which suggests that inexperienced nurses who have not received any leadership training frequently lead staff on acute wards (NPSA, 2007). Although participant hospitals in this study were focusing on strengthening the leadership culture within the organisation, neither organisation had included the role of the coordinator as part of this.

A major finding from this study suggests that the current role of the coordinator is overburdened with excessive responsibilities, prohibiting the most senior nurse on the shift from providing clinical leadership. According to nursing participants this inability to have oversight of the ward may be putting acutely unwell ward patients at risk. This finding has not been previously reported and therefore adds important new insights to what is known about the role of shift leadership as a factor affecting care of acutely unwell ward patients.

The findings from this research suggest that organisations may be unaware of the importance of the coordinator in providing shift leadership as a key to the safe care of acutely unwell ward patients. As a new contribution to the body of knowledge regarding factors affecting care of acutely unwell ward patients this finding identifies a need to examine the role of the coordinator in the provision of shift leadership and to explore training and support required by nurses who undertake this role.

\subsubsection{Organisational systems and processes of care, and culture and environment}

Three key issues emerged from the themes of organisational systems and processes of care, and culture and environments, all of which are interrelated and 
occur simultaneously. Firstly, participants perceived that the hospitals did not have clear implementation and evaluation strategies for new service initiatives implemented to support staff in their care of acutely unwell ward patients. Secondly, organisations implemented new service initiatives as a reaction to sentinel and serious events, as opposed to developing a proactive patient safety culture. Thirdly, there was a perception that too many changes were introduced at once. The following section, whilst acknowledging that these issues are inextricably linked, will discuss each of these issues independently.

In this study, executive and senior management participants from both organisations believed they fostered a strong patient safety culture through their awareness and management of clinical risk. Findings from this study demonstrate that executive management participants perceived the introduction of new service initiatives such as CCOS and EWS to ameliorate clinical risk as a key factor affecting the care of acutely unwell ward patients. The use of services such as CCOS and EWS is well reported in the suboptimal care literature as a means of supporting ward staff in their management of acutely unwell ward patients (Andrews \& Waterman, 2005; Ball, Kirby, \& Williams, 2003; Chellel Higgs, \& Scholes, 2006; Cuthbertson, Boroujerdi, McKie, Aucott, \& Prescott, 2007; Pittard, 2003; Priestley et al., 2004).

However this study suggests clear implementation and evaluation strategies are required to ensure that new service delivery changes are understood and are working effectively. For example due to lack of evaluation of the SBAR communication framework, nurses and doctors still perceived continuing problems when communicating about patient problems. As recommended by UK and Australian reports (ACSQHC, 2010; NCEPOD, 2005; NPSA, 2007) long-term organisational commitment is required to ensure the effectiveness of any patient safety service delivery change.

Findings from this study also suggest that both hospitals had reactive rather than proactive patient safety cultures. In this, new service initiatives were put into place as a response to, as opposed to prevention of, serious and sentinel events. Whilst the international literature suggests that the use of patient safety data, including incident reports and root cause analyses, should inform patient safety 
changes, findings from this study agree with reports which emphasise that organisations should consider trends and patterns rather than focusing on individual incidents (ACSQHC, 2010; NPSA, 2007).

Participants in this study described existing policies and services which were either underutilised or ineffective which may have impacted on care of acutely unwell ward patients. These included the organisation of 24/7 care (to include staffing levels after hours), management of medical outliers, the use of acuity measurement tools and the underuse of the afterhours support nurses (AHSN) in Hospital 2. This suggests that an alternative organisational approach to patient safety that includes evaluation of the efficacy of existent policies, processes and resources prior to the introduction of new service delivery changes would have a more positive impact on acutely unwell patient management. This is particularly relevant in the current healthcare climate where fiscal efficiency is high on the political agenda.

It is suggested that a key organisational process and policy area which requires review is guidance on how wards are staffed and how acutely unwell ward patients are cared for. The findings from this study and from the literature demonstrate that ward-based staff are caring for an increasingly complex inpatient population with increasing co-morbidities (Resnick \& Marcantonio, 1997; UN, 2009; van Weel \& Michels , 1997; WHO, 2003). However findings from this research suggest that ward nursing establishments have not been updated sufficiently to reflect the increasing level of patient acuity. As suggested by Ball and McElligot (2003), a review of ward nursing establishments (number, knowledge, experience) calculated in light of the level of patient dependency, the degree of clinical risk and the context in which the care is to be delivered, is required. Given the concept of Pareto optimality (where resources cannot be reallocated from one person without harming another) described in Chapter Two (section 2.4.1) any changes in resource allocation should be carefully considered to ensure that the benefits outweigh the costs. A key point determined by the findings of this research is that better use could be made of current resources rather than reallocating or requesting additional resources. 
In this study one policy for managing acuity was the availability across all cases of a patient acuity measurement tool. Use of this tool allowed daily capture of the number of sick ward patients and the level of care required. It was interesting to note the different approaches to collection and uses of this acuity data. Whilst one ward proactively used this data and was successful in gaining additional staffing resource, it was noticeable that other wards neither collected nor used the data, citing lack of specificity as the reason. These wards did not appear to use acuity data to argue their case with management for increased workforce.

The conclusion drawn from this finding was that use of accurate quantifiable patient activity and acuity data led to improved management awareness and understanding of workload and acuity issues, and resulted in the provision of additional resources. This appeared to be one way to bring perceptions of management and ward-based staff together. If similar approaches were adopted, for example to highlight to executive managers the logistic and clinical challenge for nurses and doctors in managing outliers, a more conducive environment for the safe care of sick ward patients may be created.

Participants in this study described the difficulties associated with introducing a number of new service delivery initiatives in a short period of time and in an unstable environment where staff restructuring was occurring. These findings suggest that current models of service change do not take into account the complex contemporary healthcare environment. There is a need for different approaches to change models which acknowledge the systems, processes, and human factors and account for the different perspectives of all levels of healthcare staff.

There is clear evidence from this study that participants perceived that hospital wide consultation and involvement in the introduction of patient safety initiatives did not occur. These findings also indicate a need for culture change where consultation is embedded into all stages of the development of service initiatives in order for these to be successful. Seeking the views of ward-based staff regarding the relevance and effectiveness of existing services and proposed new service delivery changes via a shared governance model would be a valid and 
beneficial strategy to achieve a hospital wide commitment to the patient safety agenda.

\subsubsection{Skills and knowledge deficit}

Findings from this study demonstrate that executive and senior management participants identify lack of skills and knowledge as a key factor affecting the care of acutely unwell ward patients; they expressed strong views on undergraduate healthcare education together with some specific concerns regarding the assessment skills required by acutely unwell ward patients. These participants believed that undergraduate nurses and doctors do not have sufficient exposure in acute care areas; this is detrimental to nurses and doctors gaining experience in caring for acutely unwell ward patients. Interestingly, this finding from a New Zealand setting adds to previous findings from UK and Australian research which recommends the review of both nursing and medical undergraduate curricula to incorporate placements in an area recognised as caring for acutely unwell patients (Cutler, 2002; Endacott et al., 2007; Wood et al., 2004).

In addition, executive management and medical participants from both hospitals identified issues with the assessment and management of acutely unwell ward patients. This was perceived as being due to a lack of specific knowledge and skills concerning nurses' ability to accurately record and interpret vital signs. These important findings concur with studies which cite the need for further education and training on the assessment and management of acutely unwell ward patients as either a key finding (Andrews \&Waterman, 2005; Buist et al., 1999; Chellel et al., 2006; Cox et al., 2006; McQuillan et al., 1998; NPSA, 2007) or recommendation (NICE, 2007).

In this study, executive management participants believed that in meeting shortfalls in knowledge and skill (evidenced by the provision of education programmes such as ALERT in Hospital 1) care for acutely unwell ward patients could be improved. This finding supports the discourse which assumes that a better educated workforce will lead to better cared for patients (Bright et al., 2004; Franklin \& Mathew, 1994; McGloin et al., 1999; McQuillan et al., 1998; NCEPOD, 2005; Wood et al., 2004). 
The fact that nursing and ward management participants in this study did not highlight skills and knowledge deficits as a factor affecting care of acutely unwell ward patients represents a further difference in viewpoint between ward-based, executive, and senior management participants. This important finding has two possible explanations: either nursing participants felt adequately educated or it may be that nursing and ward management perceived contextual issues as more important than education.

\subsection{Conclusion}

This chapter has increased understanding of factors that affect the care of acutely unwell ward patients through development and description of key organisational and environmental concepts. This multiple case study has given rich description to the understanding of impact of healthcare workforce and organisational factors that influence suboptimal care. The unique findings of this study, together with how other research has resonated with this work, have been outlined. The new contributions to knowledge will now be explored in the concluding chapter. 


\section{CHAPTER 8: CONCLUSION}

\subsection{Introduction}

There is a paucity of research that elicits factors affecting care of acutely unwell ward patients. This thesis offers important new insights into the complex factors associated with care for this group of patients. In order to profile this new learning and provide critique of the study undertaken, this chapter will systematically explore implications, strengths, and limitations of this study. In order to provide guidance on how this new knowledge can be further developed, the chapter will conclude with recommendations for further research.

\subsection{Implications of the study}

This study has potentially far reaching implications for acute care hospitals, healthcare policy, theoretical development of the concept of suboptimal care of acutely unwell ward patients and recommendations for further research. It is suggested that in order to enable improved care for acutely unwell ward patients, action is required at both ward-based and executive management level.

\subsubsection{Implications for acute care hospitals}

1. The role of the coordinator must be recognised by managerial staff as pivotal to the safe care of acutely unwell ward patients especially after hours. The results of this study show that staff perceive that in order to provide the necessary leadership and oversight of a ward during a shift, the coordinator cannot take a patient load. Executive management within both organisations need to ensure sufficient staffing to ensure that those charged with the responsibilities of this role are adequately prepared and supported as a matter of urgency. This includes providing training for the role and the development of clear expectations and accountabilities.

2. This study also demonstrates that there are cultural characteristics found on surgical wards which may be associated with the retention of staff. One such characteristic is the focus on developing relationships outside of working hours to foster effective teamwork. It would be beneficial for the organisations which 
participated in this study to look closely at those wards where a core of senior staff have been retained and determine what strategies are employed so that these can be utilised elsewhere. Having sufficient numbers of senior staff with high levels of clinical experience positively impacted on teamwork and communication within this study.

3. A review of acuity measurement tools should take place to provide all ward specialties with the opportunity to gather quantifiable data about the complexity and dependency of their patient load. A one size fits all approach is only feasible if all differences in patient populations, e.g. between surgical and medical wards, are captured. Collecting and reporting this data should become part of each organisation's quality assurance programme and should be part of the local data set for organisational review. In making this data useful to every ward specialty it could be used to inform business plans supporting acute care ward initiatives.

4. Implementing a Deteriorating Patient Group in each hospital as described in 7.3.1 may be a way of bringing together the polarised views held across hospital organisations on factors affecting the care of acutely unwell ward patients. The model postulated by the NPSA suggests that multidisciplinary membership is necessary. This multi disciplinary membership should include healthcare professionals, managers (executive and senior), educators, and a patient representative. This group could review acuity data and make recommendations to executive managers on action required, evaluate acuity service initiatives, and advise on possible future solutions to improve care in this area.

\subsubsection{Implications for healthcare policy}

1. It is important that any future health policy initiatives be informed by multiple, representative, user views. It is suggested that this include ward-based through to executive and DHB participants. Adoption of this approach will ensure that future policy is fit for purpose, fit for practice and has potential for maximum buy in. As raised in 8.4 , any further initiatives should be introduced only after full service evaluation of current suboptimal care strategies has occurred. 
2. Any future health policy directed towards improving care of acutely unwell ward patients must be cognisant of the complex contemporary healthcare environment within which it will be embedded. Suggested implementation strategies must acknowledge multiple users, multiple contexts, and multiple organisational levels in which any service change must occur.

\subsubsection{Implications for theoretical development}

1. This study has brought new understanding of suboptimal care in the context of the acutely unwell ward patient through knowledge generation of new antecedents. This multiple case study has specifically informed the concept analysis of suboptimal care through identifying a lack of shift leadership as an emergent antecedent in the New Zealand context. This is novel as the majority of theoretical work in this area to date has focussed on the defining attributes and consequences of suboptimal care.

\subsection{Strengths and limitations of the study}

Conducting a concept analysis on the term 'suboptimal care' was a strength of this study. Through systematically identifying definitions, uses, attributes, antecedents and consequences of suboptimal care the significance of the concept and its close association with caring for acutely unwell ward patients has become apparent. The concept analysis process also highlighted that previous research has focussed mainly on quantifying the occurrence of suboptimal care of acutely unwell ward patients as opposed to determining factors which may cause it. Furthermore clarifying the components which constitute the concept of suboptimal care was an effective strategy to ensure a thorough and robust research design.

A further strength of this study was inherent in the choice of multiple case study as the appropriate research approach to determine factors affecting care of acutely unwell ward patients. In this study, using three sources of data (interviews, focus groups and documentation) from multiple cases has allowed rich detail to emerge which is required to explicate such a multifaceted research problem. Furthermore using a case study approach has facilitated a thorough exploration of the context in which suboptimal care occurs demonstrating that potential solutions 
and future research should be directed at both ward and organisational level. However this is a small study, consisting of the in-depth analysis of four wards. As such, generalizing the findings beyond the four cases and two hospitals is difficult. As described in Chapter 3, proponents of case study state that analytical generalisations are one of the strengths of case study (Yin, 2003). In this case study patterns emerged which supported the theoretical framework and no rival theory emerged as a better fit during the analysis.

A strength and a limitation of this study arises from undertaking a research project in a clinical environment. The strength is that frontline staff are the most appropriate participants to describe factors affecting their daily practice. The limitation is the busy wards they work in and their availability to participate. In some of the interviews and focus groups the ward nurses and junior doctors were very busy when on duty and therefore had only a limited amount of time. Having longer with some of these participants may have allowed them to develop their stories further. This was also the case with some executive management participants who, with full schedules, restricted appointment times, which may have resulted in limited data.

Another limitation in this study was the imbalance between the numbers of house surgeons who participated between the hospitals. The large focus group of nine house surgeons in Hospital 1 provided an organisation-wide perspective that was not replicated in Hospital 2. Conversely there were no house surgeon participants at individual case level in Hospital 1, although two senior doctors did participate. Access to the large group of house surgeons in Hospital 1 was facilitated through being invited to attend a house surgeons' lunchtime meeting to describe the study and recruit potential participants. After listening to the presentation about the study the house surgeons suggested opportunistically holding the focus group there and then. Since the house surgeons had taken part in the focus group, they declined further participation in individual interviews. Despite efforts to hold a focus group for house surgeons in Hospital 2, that did not materialise. Only two house surgeons agreed to participate and were interviewed individually. Whilst this difference in participants across the cases is acknowledged, 
rigour was maintained in both sites in how participants were recruited and how data was obtained.

Finally the absence of observation as a technique for gathering data could be postulated as a limitation of this study. Both Yin (2003) and Stake (1995) encourage the use of observation (both participant and non participant) as a useful way of providing greater understanding of the case. However using observation as a method of data collection in this study was perceived as problematic specifically in relation to the issue of informed consent (for example being able to control who entered the research environment and whether they had consented to being part of the research project or not). Furthermore observation traditionally requires the researcher to spend "considerable time in the field" (Baker, 2006, p. 171) suggesting that a prolonged time period should be spent in the research environment. With the researchers choice of study sites and work commitments this was not possible therefore a pragmatic decision was taken to limit the research design to interviews, focus groups and documentation review.

\subsection{Recommendations for further research}

The factors affecting care of acutely unwell ward patients in this study are complex and multi faceted. This complexity has generated several issues and questions that warrant further exploration:

1. The role and responsibilities of the coordinator warrants further investigation. Research which investigates current strategies which prepare senior nursing staff for shift leadership is required. A mixed methods approach could be used to determine what training and support senior nurses who regularly coordinate shifts receive, and how effective this is. This could highlight areas for strategy development to help strengthen this key role.

2. Retention of senior nursing staff is more apparent in surgical areas than in medical wards. This needs to be further explored in a larger study using an ethnographic approach. Specific cultural characteristics could be identified leading to the retention of senior staff in surgical ward areas. 
3. The current study found gaps in perception of workload issues such as nurse to patient ratio and skill mix between executive management and frontline staff. The way in which organisations engage with frontline staff regarding the realities of working in medical and surgical wards is worthy of further investigation.

4. There is currently no national overview of strategies employed to reduce the incidence of suboptimal care in New Zealand. A national study which provides both an indication of service delivery changes implemented (if any) across the 20 DHBs would be useful. This study should also explore the effectiveness of strategies from a clinician's perspective. This could identify success stories and provide a resource for NZ hospitals who are still formulating their patient safety strategies.

\subsection{Conclusion}

The aim of this thesis was to explore factors affecting care of acutely unwell patients in the context of medical and surgical wards. This multiple case study of two general surgical and two general medical wards in two New Zealand hospitals has demonstrated that factors affecting care of acutely unwell ward patients are complex and multifaceted. Furthermore in this study these factors were generally not unique to individual ward environments. Perceived heavy workloads, inadequate staffing, poor communication, together with unprepared and overburdened coordinators, are all significant factors which directly affect the care of acutely unwell ward patients. Strong working relationships within and across professional groups are seen to positively impact on the clinical management of acutely unwell patients, whereas poor relationships between professional groups generates barriers to care.

This thesis confirms that the medical and surgical ward environment is challenging, due to increased patient complexity. Such patient complexity requires a skilled healthcare workforce with sufficient numbers to deliver the care required, together with organisational processes which support the healthcare workforce in providing timely and appropriate care.

This research has illustrated that polarised views exist between those who run organisations (executive managers) and those who deliver care at ward level (nurses and doctors). These differences are between perceptions of workload, 
staffing levels, perceived benefits of new service initiatives and the need for further education. This study demonstrates that organisations need to recognise that service initiatives alone are not enough to bring improvements to care of acutely unwell ward patients. Attention must be given to the inherently harder challenge of reviewing organisational policies and reorganising hospital processes to meet the demands of increasing patient acuity. In an ever more complex hospital environment, this challenge cannot be overemphasised. 


\section{APPENDICES}


APPENDIX 1: Research Protocol

\section{AN EXAMINATION OF THE FACTORS INFLUENCING CARE IN ACUTELY UNWELL WARD PATIENTS}

\section{Background}

The proposed study will examine factors affecting care of acutely unwell ward patients. Reviewed literature has highlighted that patients in general surgical and medical wards can become acutely unwell, with deranged physiological markers such as respiratory rate, blood pressure and heart rate. These signs are often missed or, if they are observed, then inappropriate or ineffective action is taken. This phenomenon has been labelled 'suboptimal care'. Suboptimal care can have catastrophic effects with patients noted to die unexpectedly or have an unexpected admission to Intensive Care. This research will explore health care staff's experiences of the issues they encounter when caring for the acutely unwell ward patient. A particular focus of this study will be exploration of organisational structures and processes. By examining what these factors are, recommendations can be made to improve care of these patients.

\section{Design}

A case study methodology will be used to compare two hospitals in NZ. One metropolitan hospital and one regional hospital have been chosen so that similarities and differences can be examined. The study has three data sources.

1. Within each hospital two wards (one general medical and one general surgical) will be identifed where all the nurses, doctors and managers who agree to participate will be interviewed either individually or in focus groups. The focus groups will be organised within professions (e.g. nurses together, doctors together).

2. The Chief Operating Officer, Chief Medical Advisor and Director of Nursing will be approached to participate in one to one semi structured interviews in order to provide a broader organisational perspective.

3. Documentation relevant to the study such as nursing rosters, patient acuity and skill mix data will be reviewed. 


\section{Gaining access}

- Contact will be made with the Directors of Nursing from each hospital via email. A synopsis of the study will be sent as an attachment. A follow up meeting/telephone conversation will be arranged. The DoN will be asked to recommend specific wards to be studied. For wards to be eligible for the study they must have a history of caring for acutely unwell patients. Wards which would not be eligible would be those areas which are considered to be highly specialized such as orthopaedic, cardiothoracic, renal and neurological wards. The rationale for excluding specialty areas is based on prior research in this field using general wards as the research setting and the underlying assumption that this phenomenon is predominantly an issue in these areas.

- Complete locality assessment procedures as per individual hospital

- Make contact with clinical nurse managers (CNMs) from surgical and medical wards. Send synopsis of research

- Arrange dates for research to take place

\section{Fieldwork}

When access is granted participant recruitment will commence. The participants will be a purposive sample taken from the cases under investigation. The aim is to conduct focus groups and interviews with registered nursing, medical and managerial staff from the case. Posters advertising the study will be displayed in the selected ward areas inviting all registered nurses, doctors and ward managers to participate. It is envisaged that the sample will include nursing and medical staff of all levels of experience to provide a true reflection of the health practitioner workforce within the ward.

\section{Participant recruitment strategies}

- Sit in coffee room to discuss study with ward staff during coffee and meal breaks.

- Write entry in ward communication book about study

- Sit in handover and discuss study after handover finished

- Present at Registrars meeting

- Present at post take meeting

- Present at grand round

- Present at ward meeting

\section{Data Collection}

- Demographic data of the study sample will be collected to include occupation, age, gender, years of experience and post graduate educational qualifications. 
- The researcher will conduct focus groups with nurses, doctors and managers.

- One to one interviews will be offered to staff that do not wish to participate in focus groups but would like participate in the study. Questions within the interview will be open ended and structured around two key areas;

"Can you tell me about caring for an acutely unwell patient which was a positive experience for you? What factors made that experience a positive one?" and

"Can you tell me about another experience when you cared for an acutely unwell patient that did not go as well. What factors made that experience go less well? What do you think could be done to improve that?"

- Each focus group/interview will last for approximately one hour. All focus groups/interviews will be tape recorded and then transcribed by the researcher.

- The transcriptions will be read and reread thereby using an iterative and inductive process to formulate sub categories for further analysis.

- Key stakeholders such as the Director of Nursing, the Chief Operating Officer and the Hospital Chief Medical Officer will be approached to give 1:1 interviews in order to provide a broader organizational perspective.

\section{Document Review}

In order to provide organisational context to the study information pertaining to the hospital activity and services provided will be collected. Ward level documentation to be reviewed will include ward staffing data to include nursing rosters, staff turnover, sickness and annual leave data, casual and agency nurse use (when kept). Additional documentation regarding patient acuity data, ward meeting minutes, model of care and ward educational activities. Additional data to be reviewed will include the presence of a Critical Care Outreach Service, Medical Emergency Team, Early Warning Score or other patient safety initiatives.

\section{Ethical Considerations}

Participation in the study will be voluntary. Once staff have demonstrated willingness to participate an information sheet and consent form will be given to them for signing. Participants will be assured that all information gathered though the focus groups/interviews will be confidential. Participants will be assured that 
they are able to withdraw from the study at any time and will be assured that any data they may have contributed will not be utilized as part of the study and will be destroyed. Any reviewed documentation will be anonymised.

\section{Ethical Approval}

Ethical approval has been granted by the Multi Region Ethics committee reference number MEC/O9/26/EXP

\section{Study timeline}

October 2009- Fieldwork Regional Hospital (6 weeks)

Mid November -2009 Fieldwork Metropolitan Hospital (6 weeks)

\section{Research Outcomes}

Through understanding staff experiences when caring for the acutely unwell patient in ward areas it is intended to inform organisational structure and processes with the aim of improving patient outcome.

\section{Dissemination}

1. As a report to participating hospitals

2. Publication of sections within peer reviewed journals 
APPENDIX 2: Ethical Approval

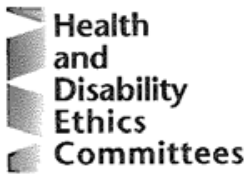

\author{
Multi-region Ethics Committee \\ Minisity of Health \\ Level 2, 1-3 The Terrace \\ PO Box 5013 \\ Wellington \\ Phone (04) 4700655 \\ (04) 4700646 \\ Fax (04) 4962191 \\ Email: multiregion_ethicscommittee@moh.govt.nz
}

1 May 2009

Sarah Quirke

Graduate School of Nursing, Midwifery and Health

81 Fairlie Tce

Kelburn

Wellington

Dear Sarah

An examination of factors infiuencing care of acutely unwell ward patients Lead Investigator: Sarah Quirke

\section{MEC/09/26/EXP}

The above study has been given ethical approval by the Multi-region Ethics Committee.

Approved Documents

- Research Proposal

- Letter of invitation

- Information sheet and consent form Version 1 for DHB stakeholder interviews

- Information sheet and consent form Version 1 for DHB Focus Group Participants

- Advertisement entitled "Research Participants Required"

\section{Accreditation}

The Committee involved in the approval of this study is accredited by the Health Research Council and is constituted and operates in accordance with the Operational Standard for Ethics Committees, April 2006.

\section{Progress Reports}

The study is approved until September 2010. The Committee will review the approved application annually and notify the Principal Investigator if it withdraws approval. It is the Principal Investigator's responsibility to forward a progress report covering all sites prior to ethical review of the project in April 2010. The report form is available at $h$ ttp: $/ / m w w$.ethicscommittees. health.govt.nz. Please note that failure to provide a progress report may result in the withdrawal of ethical approval. A final report is also required at the conclusion of the study.

Amendments

It is also a condition of approval that the Committee is advised of any adverse events, if the study does not commence, or the study is altered in any way, including all documentation eg advertisements, letters to prospective participants.

Please quote the above ethics committee reference number in all correspondence.

It should be noted that Ethics Committee approval does not imply any resource commitment or administrative facilitation by any healthcare provider within whose facility the research is to

Administered by the Ministry of Health $\quad$ Approved by the Health Research Council $\quad$ http:I/Www.ethicscommittees.health.govt.nz


carried out. Where applicable, authority for this must be obtained separately from the appropriate manager within the organisation.

Yours sincerely

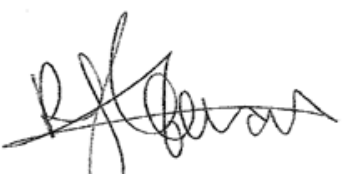

Rebecea Stewart

Multi-region Ethics Committee Administrator

Email: rebecca_stewart@moh.govt.nz 
APPENDIX 3: Participant Information Sheet for Stakeholder Interviews

TE WHARE WĀNANGA O TE ŪPOKO O TE IKA A MĀUI

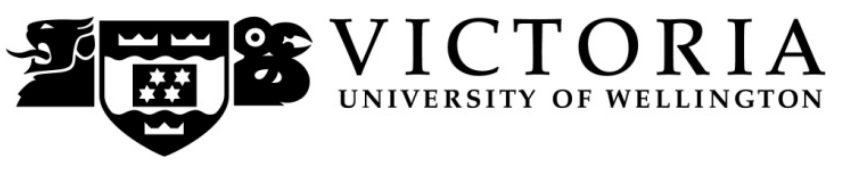

Participant Information Sheet for DHB Stakeholder Interviews

RESEARCH PROJECT: AN EXAMINATION OF THE FACTORS INFLUENCING CARE IN ACUTELY UNWELL WARD PATIENTS

The purpose of this study is to improve patient outcomes and the nursing experience of caring for acutely unwell ward patients by studying the factors which influence the care of acutely unwell patients. Research has highlighted that patients in general surgical and medical wards can become acutely unwell, with deranged physiological markers such as respiratory rate, blood pressure and heart rate. These signs can be missed or if they are observed, then inappropriate or ineffective action may be taken. There is little research which has attempted to determine why this occurs. This study is in three parts and each of the three parts will be conducted in two different hospitals.

Part 1 - Nurses, Doctors and Managers who work within general surgical and medical wards will be invited to participate in focus groups.

Part 2 - The Directors of Nursing, Chief Medical Officer and Chief Operating Officer from each hospital will be invited to participate in one to one interviews.

Part 3 - Documentation such as nursing rosters, patient acuity tools, nurse patient ratio and skill mix and nursing establishment data will be examined.

The study design aims to facilitate a multi dimensional view of the factors which influence the care of acutely unwell ward patients.

Ethics approval has been granted by the Multi Region Ethics Committee of New Zealand via the expedited review process. (MEC/09/26/EXP).

I am contacting key stakeholders such as yourself to invite you to participate in an interview of no more than 60 minutes duration, about the care of acutely unwell 
patients within your organisation. The focus of the interview will include your perspective on how acutely unwell patients are cared for within your organisation. Topics for discussion are likely to include the factors which you believe enhance the process, factors which make it difficult, resourcing and role and responsibilities of the organisation and the individual practitioners in caring for this patient group. The interview will take place at a negotiated time and venue. Your participation is voluntary.

With your permission the interview will be audio taped and the tapes transcribed using transcription software. The audiotapes will be stored in a lockable filing cabinet in the Graduate School of Nursing, Midwifery and Health at Victoria University of Wellington. All research findings will be non-identifiable and will form part of the final PhD submission. All data will be held for five years following completion of the research and then destroyed.

If you do agree to take part you are free to withdraw from the study at any time, without having to give a reason.

If you have any questions or would like to receive further information about the project please contact:

\section{Principal Investigator}

Sara Quirke PhD candidate Lecturer in Clinical Nursing Graduate School of Nursing Midwifery and Health Victoria University of Wellington 044636151

\section{Supervisor}

Dr Rose McEldowney

Associate Professor in Nursing

Graduate School of Nursing Midwifery and Health

Victoria University of Wellington

044636155 
APPENDIX 4: Participant Information Sheet for Stakeholder Focus Groups

TE WHARE WĀNANGA O TE ŪPOKO O TE IKA A MĀUI

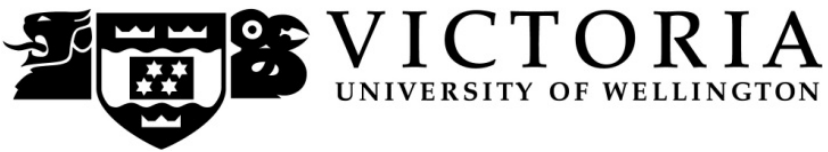

Participant Information Sheet for DHB Focus Group Participants

RESEARCH PROJECT: AN EXAMINATION OF THE FACTORS INFLUENCING

CARE IN ACUTELY UNWELL WARD PATIENTS

The purpose of this study is to improve patient outcomes and the nursing experience of caring for acutely unwell ward patients by studying the factors, which influence the care of acutely unwell patients. Research has highlighted that patients in general surgical and medical wards can become acutely unwell, with deranged physiological markers such as respiratory rate, blood pressure and heart rate. These signs can be missed or if they are observed, then inappropriate or ineffective action may be taken. There is little research which has attempted to determine why this occurs. This study is in three parts and all three parts will be conducted in two different hospitals.

Part 1 - Nurses, Doctors and Managers who work within general surgical and medical wards will be invited to participate in focus groups.

Part 2 - The Directors of Nursing, Chief Medical Officer and Chief Operating Officer from each hospital will be invited to participate in one to one interviews.

Part 3 -Documentation such as nursing rosters, patient acuity tools, nurse patient ratio, skill mix and nursing establishment data will be examined.

The study design aims to facilitate a multi dimensional view of the factors which influence the care of acutely unwell ward patients.

Ethics approval has been granted by the Multi Region Ethics Committee of New Zealand via the expedited review process (MEC/09/26/EXP).

I am contacting key stakeholders such as yourself to invite you to participate in a focus group of no more than 60 minutes duration, about the care of acutely unwell patients within your ward. Ground rules including those of confidentiality will be 
agreed with each group. The topics for discussion are likely to include recalling and discussing occasions when you have cared for acutely unwell patients. A specific focus will be sharing your experiences of what went well on those occasions and what influenced how that patient was cared for. In order to enhance patient care it is helpful to explore care episodes which went well to extract what particular factors were present that made the experience successful. Likewise it is also helpful to explore those episodes that did not go as well to determine why. The focus group session will take place at a negotiated time and venue. Your participation is voluntary.

With your permission the focus group will be audio taped and the tapes transcribed using transcription software. The audiotapes will be stored in a lockable filing cabinet in the Graduate School of Nursing, Midwifery and Health at Victoria University of Wellington. All research findings will be non-identifiable and will form part of the final PhD submission. All data will be held for five years following completion of the research and then destroyed.

If you do agree to take part you are free to withdraw from the study at any time, without having to give a reason.

If you have any questions or would like to receive further information about the project please contact:

\section{Principal Investigator}

Sara Quirke PhD candidate

Lecturer in Clinical Nursing

Graduate School of Nursing Midwifery and Health

Victoria University of Wellington

044636151

\section{Supervisor}

Dr Rose McEldowney

Associate Professor in Nursing

Graduate School of Nursing Midwifery and Health

Victoria University of Wellington

044636651 
TE WHARE WĀNANGA O TE ŪPOKO O TE IKA A MĀUI

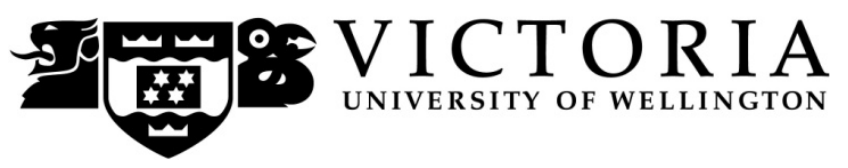

Participant Consent Form: DHB Stakeholder Interviews

RESEARCH PROJECT: AN EXAMINATION OF THE FACTORS INFLUENCING

CARE IN ACUTELY UNWELL WARD PATIENTS

I have read and understood the information sheet asking for participants to take part in an interview. I understand the interview will be about sharing my perspectives in terms of how acutely unwell patients are cared for within this organisation and that the topics for discussion are likely to include the factors which enhance the process, factors which make it difficult, resourcing and role and responsibilities of the organisation and the individual practitioners in caring for this patient group.

I have had an opportunity to ask questions and have them answered to my satisfaction. I have had time to consider whether to take part. I understand that I may withdraw myself (or any information I have provided) from this project (before data collection and analysis is complete) without having to give reasons.

I consent to the interview being audio taped.

I understand that any information I provide will be kept confidential to the researcher and the researcher's supervisor.

I understand that my participation in this study is confidential and that no material which could identify me will be used in this study.

I understand that the tape recording of interviews will be electronically wiped at the end of the project. I understand that data will be stored safely in the 
researchers locked filing cabinet in her office in the Graduate School of Nursing Midwifery and Health at Victoria University of Wellington.

I would like to receive a summary of the result of this research when it is completed

Yes No (Please circle)

I (full name) consent to take part in

this research

Signed:

Date:

\section{Principal Investigator}

Sara Quirke

Lecturer in Clinical Nursing

Graduate School of Nursing,

Midwifery and Health

Victoria University of Wellington

e-mail sara.quirke@vuw.ac.nz

Telephone 044636151

\section{Supervisor}

Dr Rose McEldowney

Associate Professor in Nursing

Graduate School of Nursing

Midwifery and Health

Victoria University of Wellington

e-mail rose.mceldowney@vuw.ac.nz

Telephone 044636651 
TE WHARE WĀNANGA O TE ŪPOKO O TE IKA A MĀUI

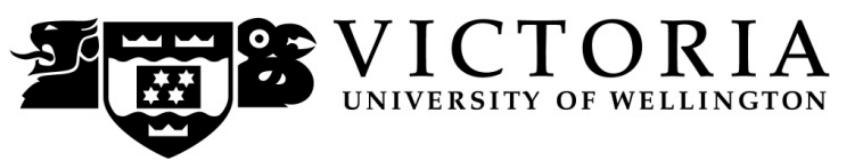

Participant Consent Form: DHB Focus Group

RESEARCH PROJECT: AN EXAMINATION OF THE FACTORS INFLUENCING

CARE IN ACUTELY UNWELL WARD PATIENTS

I have read and understood the information sheet asking for participants to take part in focus groups. I understand the focus group session will be about my experiences of caring for acutely unwell patients within the ward area and that topics for discussion are likely to include recalling and discussing factors which have influenced how I have cared for this patient group.

I have had an opportunity to ask questions and have them answered to my satisfaction. I have had time to consider whether to take part. I understand that I may withdraw myself from this project (before data collection and analysis is complete) without having to give reasons.

I understand that I will be part of a focus group where my opinions will be equally important as everyone else's. I consent to the focus group session being audio taped.

I understand that any information I provide will be kept confidential to the researcher and the researcher's supervisor.

I understand that my participation in this study is confidential and that no material which could identify me will be used in this study.

I understand that the tape recording of focus groups will be electronically wiped at the end of the project. I understand that data will be stored safely in the 
researchers locked filing cabinet in her office in the Graduate School of Nursing Midwifery and Health at Victoria University of Wellington.

I would like to receive a summary of the results of this research when it is completed

Yes No (Please circle)

। (full name) consent to take part in this research

Signed:

Date:

\section{Principal Investigator}

Sara Quirke Lecturer in Clinical Nursing Graduate School of Nursing, Midwifery and Health Victoria University of Wellington e-mail sara.quirke@vuw.ac.nz Telephone 044636151

\section{Supervisor}

Dr Rose McEldowney Associate Professor in Nursing Graduate School of Nursing Midwifery and Health Victoria University of Wellington e-mail rose.mceldowney@vuw.ac.nz Telephone 044636651 


\section{REFERENCES}

Aiken, L. H., Clarke, S.P., Sloane, D.M., Sochalski, J., \& Silber, J.H. (2002). Hospital nurse staffing and patient mortality, nurse burnout, and job dissatisfaction. Journal of the American Medical Association, 288(16), 1987-1993.

Allen, D., \& Cloyes, K. (2005). The language of 'experience' in nursing research. Nursing Inquiry, 12(2), 98-105.

Andrews, T., \& Waterman, H. (2005). Packaging: a grounded theory of how to report physiological deterioration effectively. Journal of Advanced Nursing, 52(5), 473-481.

Askoxford.com. (2009). Retrieved from http://www.askoxford.com/concise oed/suboptimal?view=uk

Australian Commission on Safety and Quality in Health Care. (2010). National consensus statement: Essential elements for recognising and responding to clinical deterioration. Sydney: ACSQHC. Retrieved from http://www.safetyandquality.gov.au/internet/safety/publishing.nsf/Content LEB5349066738C24CCA2575E70026C32A/\$SFile/national consensus statem $\underline{\text { ent.pdf }}$

Baker, L. (2006) Observation: A complex research method. Library Trends, 55 (1),171-189

Bagshaw, S. M., Mondor, E. E., Scouten, C., Montgomery, C., Slater-MacLean, L., Jones, D. A., Bellomo, R., Gibney, R.T.N. (2010). A Survey of Nurses' Beliefs About the Medical Emergency Team System in a Canadian Tertiary Hospital. American Journal of Critical Care, 19(1), 74-83. doi: 10.4037/ajcc2009532

Ball, C., Kirby, M., \& Williams, S. (2003). Effect of the critical care outreach team on patient survival to discharge from hospital admission to critical care: non randomised population based study. British Medical Journal, 327, 10141016.

Ball, C., McElligot, M. (2003). Realising the potential of critical care nurses: an exploratory study of the factors that affect and comprise the nursing contribution to the recovery of critically ill patients. Intensive and Critical Care Nursing, 19, 226-238. doi: 10.1016/S0964-3397(03)00054-5

Baldwin, M. A. (2008). Concept analysis as a method of inquiry. Nurse Researcher, 15(2), 49-58. 
Baxter, P., \& Jack, S. (2008). Qualitative case study methodology: Study design and implementation for novice researchers. The Qualitative Report, 13(4), 544559.

Bell, M. B., Konrad, D., Granath, F., Ekbom, A., \& Martling, C.R. (2005). Prevalence and sensitivity of MET-criteria in a Scandinavian University Hospital. Resuscitation, 70, 66-73. doi: 101.1016/j.resuscitation.2205.11.011

Bion, J. F. H., \& Heffner J.E. (2004). Challenges in the care of the acutely ill. The Lancet, $363970-977$.

Brennan, M. (1997). A concept analysis of consent. Journal of Advanced Nursing, 25(3), 477-484.

Bright, D., Walker, W., \& Bion, J. (2004). Clinical Review: Outreach - a strategy for improving the care of the acutely ill hospitalised patient Critical Care 8, 3340.

Brownstein, B. (1980) Pareto Optimality, External Benefits and public goods: A subjectivist approach. The Journal of Libertarian Studies 4(1), 93 - 106

Bryar, R. M. (2000). An examination of case study research. Nurse Researcher, 7(2), 61-78.

Buchanan, D., Boddy, D., \& McCalman, J. (1988). Getting in, getting on, getting out and getting back. In A. Bryman (Ed.), Doing research in Organizations. London: Routledge.

Buist, M. D., Jarmalowski, E., Burton, P.R., Bernard, S.A., Waxman, B.P., \& Anderson, J. (1999). Recognising clinical instability in hospital patients before cardiac arrest or unplanned admission to intensive care. Medical Journal of Australia, 171, 22-25.

Buist, M., Bernard, S., Ngyuen, T.V., Moore, G., \& Anderson, J. (2004). Association between clinically abnormal observations and subsequent in-hospital mortality: a prospective study. Resuscitation, 62, 137-141. doi: 10.10106.j/resuscitation.2004.03.005

Burnard, P. (2005). Interviewing. Nurse Researcher, 13(1), 4-6.

Byers, T. H., \& Waterman, M.S. (1984). Determining all optimal and near optimal solutions when solving all shortest path problems by dynamic programming. Operations Research, 32(6), 1381-1384.

Cambridge Dictionaries online. Retrieved from http://dictionary.cambridge.org/results.asp?searchword=suboptimal\&x=0\& $\mathrm{y}=0>$ 
Charmaz, K. (2006). Constructing grounded theory: A practical guide through qualitative research. Los Angeles: Sage.

Chellel, A., Fraser, J., Fender, V., Higgs, D., Buras-Rees, S., Hook, L., et al. (2002). Nursing observations on ward patients at risk of critical illness. Nursing Times, 98(46), 36-39.

Chellel, A., Higgs, D., \& Scholes, J. (2006). An evaluation of the contribution of critical care outreach to the clinical management of the critically ill ward patient in two acute NHS trusts. Nursing in Critical Care, 11(1), 42-51.

Cioffi, J. (2000). Recognition of patients who require emergency assistance: a descriptive study. Heart Lung: Journal of Acute Critical Care, 29(4), 108-114.

Clarke, S. P., \& Aiken, L.H. (2003). Failure to rescue. American Journal of Nursing, 103(1), 42-47.

Clarke, S. P., \& Aiken, L.H. (2006). More nursing, fewer deaths. Quality and Safety in Healthcare, 15, 2-3. doi: 10.1136/qshc.2005.017343

Considine, J., \& Botti, M. (2004). Who, when and where? Identification of patients at risk of an in-hospital adverse event: Implications or nursing practice. International Journal of Nursing Practice, 10, 21-31.

Coolican, H. (1999). Research methods and statistics in psychology $\left(2^{\text {nd }}\right.$ ed.). London: Hodder \& Stoughton.

Costanza, V. (2007). Parametric uncertainty and disturbance attenuation in the suboptimal control of a non-linear electrochemical process. Optimal Control Applications and Methods, 28, 209-228. doi: 10.1002/oca.798

Cox, H., James, J., \& Hunt, J. (2006). The experiences of trained nurses caring for critically ill patients within a general ward setting. Intensive and Critical Care Nursing, 22, 283-293.

Curtis, E., \& Redmond, R. (2007). Focus groups in nursing research. Nurse Researcher, 14(2), 25-37.

Cuthbertson, B. H., Boroujerdi, M., McKie. L., Aucott, L., \& Prescott, G. (2007). Can physiological variables and early warning scoring systems allow early recognition of the deteriorating surgical patient? Critical Care Medicine, 35(2), 402-409. doi:10.1097/01.CCM.0000254826.19520.87

Cutler, L. R. (2002). From ward based critical care to educational curriculum 2: a focused ethnographic case study. Intensive and Critical Care Nursing, 18, 280-291. 
Department of Health. (2000). Comprehensive Critical Care: A review of adult critical care services. London: Department of Health.

Department of Health. (2009). What is the European working time directive? Retrieved $1^{\text {st }}$ May 2009, from http://www.dh.gov.uk/en/Managingyourorganisation/Workforce/Workforc eplanninganddevelopment/Europeanworkingtimedirective/DH 077304

Derham, C. (2007). Achieving comprehensive critical care. Nursing in Critical Care, 12(3), 124-131.

Denzin, N. K., Lincoln, Y.S (Ed.). (1994). Handbook of Qualitative Research. Thousand Oaks, CA: Sage.

Donabedian, A. (2005). Evaluating the Quality of Medical Care. The Millbank Quarterly, 83(4) 691-729.

Duncan, C., Cloutier, J. D., \& Bailey, P. H. (2007). Concept analysis: the importance of differentiating the ontological focus. Journal of Advanced Nursing, 58(3), 293-300.

Endacott, R., Kidd., T., Chaboyer, W., \& Edington, J. (2007). Recognition and communication of patient deterioration in a regional hospital: A multi methods study. Australian Critical Care, 20, 100-105. doi:

10.1016/j.aucc.2007.05.002

Ferlie, E. B., \& Shortell, S. M. (2001). Improving the Quality of Health Care in the United Kingdom and the United States: A Framework for Change. Milbank Quarterly, 79(2), 281-315. doi: 10.1111/1468-0009.00206

Franklin, C., \& Mathew, J. (1994). Developing strategies to prevent in-hospital cardiac arrest: Analysing responses of physicians and nurses in the hours before the event Critical Care Medicine 22(2), 244-247.

Gangeness, J. E., \& Yurkovich, E. (2006). Revisiting case study as a nursing research design. Nurse Researcher, 13(4), 7-17.

Gao, H., McDonnell, A., Harrison, D., Moore, T., Adam, S., Daly, K., ... \& Harvey ,S.I. (2007). Systematic review and evaluation of physiological track and trigger warning systems for identifying at-risk patients on the ward. Intensive Care Medicine, 33(4), 667-679. doi: 10.1007/s00134-007-0532-3

Gearing, R., Mian I., Barber, J., \& Ickowicz, A. (2006). A Methodology for Conducting Retrospective Chart Review Research in Child and Adolescent Psychiatry. Journal of the Canadian Academy of Child and Adolescent Psychiatry 15(3), 126-134. 
Gershenson, D. (2001). Why American women are not receiving state of the art gynaecologic cancer care. The Cancer Journal, 7(6), 450-457.

Gibbard, A. (1984). Health Care and the Prospective Pareto Principle. Ethics, 94(2), 261-282.

Goldhill, D. R., White, S.A., \& Sumner, A. (1999). Physiological values and procedures in the $24 \mathrm{~h}$ before ICU admission from the ward. Anaesthesia 54 529-534.

Graneheim, U. H., \& Lundman, B. (2004). Qualitative content analysis in nursing research: concepts, procedures and measures to achieve trustworthiness. Nurse Education Today, 24(2), 105-112.

Hancock, D., \& Algozzine, B. (2006). Doing case study research. New York: Teachers College Press.

Hess, D. (2004). Retrospective Studies and Chart Reviews. Respiratory Care, 49(10), 1171-1174.

Hillman, K., Chen, J., Cretikos, M., Bellomo, R., Brown, D., Doig, G., Finnfer, S., Flabouris, A. (2005). Introduction of the Medical Emergency team (MET) system: a cluster randomised controlled trial. Lancet, 365, 2091-2097.

Hodgetts, T. J., Kenward, G., Vlackonikolis, I., Payne, S., Castle, N., Crouch, R.,... \& Shaikh, L. (2002). Incidence, location and reasons for avoidable in-hospital arrest in a district general hospital. Resuscitation 54 115-123.

Hupcey, J. E., \& Penrod, J. (2005). Concept analysis: Examining the state of the science. Research and Theory for Nursing Practice: An International Journal, 19(2), 197-208.

Jones, D., Baldwin, I., Mclnyre, T., Story, D., Mercer., I., Miglic, A., Goldsmith, D., Bellomo, R. (2006). Nurses attitude to a medical emergency team service in a teaching hospital. Quality and Safety in Health Care, 15 427-432. doi 10.1136/qshc.2005.016956

Jones, L., King, L., \& Wilson, C. (2009). A literature review: factors that impact on nurses' effective use of the Medical Emergency Team (MET). Journal of Clinical Nursing, 18(24), 3379-3390. doi: 10.1111/j.1365-2702.2009.02944.x

Jones, C., \& Lyons, C. (2004). Case study: Design? Method? Or comprehensive strategy. Nurse Researcher, 11(3), 70-76.

Joo, K., Lee, J., Kim, I., Jong Lee, S., \& Lee, J. (2008). Multiple sequence alignment by conformational space annealing. Biophysical Journal, 95(10), 4813-4819. doi: 10.1529/biophysj.108.129684 
Kause, J., Smith, G., Prytherch, D., Parr, M., Flabouris, A., \& Hillman, K. (2004). A comparison of antecedents to cardiac arrests, deaths and emergency intensive care admissions in Australia and New Zealand, and the United Kingdom - the ACADEMIA study Resuscitation, 62, 275-282.

Koch, T., \& Harrington, A. (1998). Reconceptualising rigour: the case for reflexivity. Journal of Advanced Nursing, 28(4), 882-890.

Konijnenberg, A., De Graeff-Meeder, E., Kimpen, J., van Der Hoeven, J., Buitelaar, J., \& Uiterwaal, C. (2004). Children with unexplained chronic pain: Do paediatricians agree regarding diagnostic approach and presumed primary cause. Pediatrics, 114(5), 12201226.

Lawless, J., Wan, L., \& Zeng, I. (2010). Patient care rationed as nurses struggle under heavy workloads - survey. Kai Tiaki, 16(7), 16-18.

Lee, A., Bishop, G., Hillman, K., \& Daffurn K. (1995). The Medical Emergency Team. Anaesthetic Intensive Care, 23, 183-186.

Long, T., \& Johnson, M. (2000). Rigour, reliability and validity in qualitative research. Clinical Effectiveness in Nursing, 4(1), 30-37.

Massey, D., Aitken, L.M., \& Chaboyer, W. (2008). What factors influence suboptimal care in the acutely ill ward patient. Australian Critical Care, 21, 127-140.

McArthur-Rouse, F. (2001). Critical care outreach services and early warning scoring systems: a review of the literature. Journal of Advanced Nursing, 36(5), 696704.

McDonnell, A., Lloyd Jones, M., \& Read, S. (2000). Practical considerations in case study research: the relation between methodology and process. Journal of Advanced Nursing, 32(2), 383-390.

McGaughey, J., Allerdice, F., Fowler, R., Kapila, A., Mayhew, A., \& Moutray, M. (2007). Outreach and Early Warning Systems (EWS) for the prevention of intensive care admission and death of critically ill adult patients on general wards. Cochrane Database of Systematic Reviews (3), 1-20. doi: $10.1002 / 14651858$

McGloin, H., Adam, S., \& Singer, M. (1999). Unexpected deaths and referrals to intensive care of patients on general wards. Are some cases potentially avoidable? Journal of the Royal College of Physicians London, 33, 255-259.

McQuillan, P., Pilkington, S., Allan, A., Taylor, B., Short, A., Morgan, G.,...\& Smith, G. (1998). Confidential inquiry into quality of care before admission to intensive care. British Medical Journal, 316, 1853-1858. 
MedicineNet.com. (2009) Retrieved from

http://search.medicinenet.com/search/search_results/default.aspx?Search what $=1 \&$ query=SUBOPTIMAL+CARE\& 1 . $x=0 \&|1 . y=0 \&| 1=$ Search

MedlinePlus. (2009) Retrieved from

http://www.merriam-webster.com/medlineplus/suboptimal

Miles, M., \& Huberman, A. (1994). Qualitative data analysis (2nd ed.). Thousand Oaks, CA: Sage.

Morrice, A., \& Simpson, H.J. (2007). Identifying level one patients: A cross sectional survey on an inpatient hospital population. Intensive and Critical Care Nursing, 23, 23-32.

Morse, J. M., \& Field, P.A. (1995). Qualitative Research Methods for Health Professionals (2nd ed.). Thousand Oaks, CA: Sage.

Morse, J., Barrett, M., Mayan, M., Olson, K., \& Spiers, J. (2008). Verification Strategies for Establishing Reliability and Validity in Qualitative Research. International Journal of Qualitative Methods, 1(2). Retrieved January 5, 2011, from http://ejournals.library.ualberta.ca/index.php/IJQM/article/view/4603/375 $\underline{6}$

Murch, P., \& Warren, K. (2001). Developing the role of critical care liaison nurse. Nursing in Critical Care, 6(5), 221-225.

National Confidential Enquiry into Patient Outcome and Death. (2005). An acute problem? NCEPOD. London.

National Confidential Enquiry Into Patient Outcome And Death. (2009). Caring to the end? NCEPOD. London.

National Institute for Health and Clinical Excellence. (2007). Acutely ill patients in hospital: Recognition and response to acute illness in hospital. National Institute for Health and Clinical Excellence. London.

National Patient Safety Agency. (2007). Recognising and responding appropriately to early signs of deterioration in hospitalised patients. Retrieved from http://www.npsa.nhs.uk/nrls/alerts-and-directives/directivesguidance/acutely-ill-patient/deterioration-in-hospitalised-patients/

New Zealand Residents Doctors Association (2011) Collective Agreement. 28 March 2011 - 31 March 2012. Retrieved from http://www.aucklanddoctors.co.nz/media/51376/nzrda20\%20dhb\%20rmo\%20meca\%202011-2012.pdf 
Okumus, F., Altinay, L., \& Roper, A. (2006). Gaining access for research; Reflections from experience. Annals of Tourism Research, 34(1), 7-26. doi:

10.1016/j.annals.2006.07.006

Paley, J. (1996). How not to clarify concepts in nursing. Journal of Advanced Nursing, 24, 572-578.

Palmer, N., Appleton, B., \& Rodrigues, E. (2003). Specialist nurse-led intervention in outpatients with congestive heart failure. Disease Management and Health Outcomes, 11(11), 693-698.

Patton, M. Q. (1999). Enhancing the quality and credibility of qualitative analysis. Health Services Research, 34(5), 1189-1208.

Pittard, A. J. (2003). Out of our reach? Assessing the impact of introducing a critical care outreach service. Anaesthesia, 58(9), 882-887.

Polifroni, E.C., Welch, M. (1999) Perspectives on philosophy of science in nursing. Philadelphia: Lippincott.

Platt, J. (1992). 'Case study' in American Methodological thought. Current Sociology, 40(17), 17-48.

Plowright, C., O'Riordan, B., Scott, G. (2005). The perception of ward-based nurses seconded into an Outreach service. Nursing in Critical Care, 10(3), 143-149.

Priestley, G., Watson, W., Rashidan, A., Mozley, C., Russell, D., Wilson, J., Cope, J., Hart, D., Kay, D., Cowley, K., \& Pateraki, J. (2004). Introducing Critical Care Outreach: a ward randomised trial of phased introduction in a general hospital. Intensive Care Medicine, 30, 1398-1404. doi: 10.1007/s00134-004-2268-7.

Quality Improvement Committee (2009). Serious and Sentinel Events in New Zealand Hospitals 2007 - 2008. Wellington: The Quality Improvement Committee.

Rafferty, A. M., Clarke, S.P., Coles, J., Ball, J., James, P., McKee, M., \& Aiken., L.H. (2007). Outcomes of variation in hospital nurse staffing in English hospitals; Cross sectional analysis of survey data and discharge records. International Journal of Nursing Studies, 44, 175-182.

Resnick, N. M., \& Marcantonio, E. R. (1997). How should clinical care of the aged differ? Lancet, 350 (9085), 1157.

Reynolds, S., Cardinal, P., \& Baxter, A. (2005). Reaching out beyond the ICU. Critical Care Rounds, 6(3), 1-6. 
Richards, L. (2009). Handling qualitative data: A practical guide. Thousand Oaks, California: Sage.

Risjord, M. (2009). Rethinking concept analysis. Journal of Advanced Nursing, 65(3), 684-691. doi: 10.1111/j.1365-2648.2008.04903.x

Robson, W. (2002). An evaluation of the evidence base related to critical care outreach teams - 2 years on from Comprehensive Critical Care. Intensive and Critical Care Nursing, 18, 211-218.

Rodgers, B. L. (1989). Concepts, analysis and the development of nursing knowledge: The evolutionary cycle. Journal of Advanced Nursing, 14, 330335.

Ryan, H., Cadman, C., \& Hann, L. (2004). Setting standards for assessment of ward patients at risk of deterioration. British Journal of Nursing (BJN), 13(20), 1186-1190.

Sandelowski, M. (2002). Re-embodying qualitative enquiry. Qualitative Health Research, 12(1), 104-115.

Schein, R. M. H., Hazday, N., Pena, M,. Ruben, B.H., \& Sprung, C.L. (1990). Clinical antecedents to in-hospital Cardiopulmonary arrest. Chest 98 (6), 1388-1392.

Seward, E., Greig, E., Preston, S., Harris, R.A., Borrill, Z., Wardle, T.D., Burnham, R., Driscoll, P., Harrison, B., Lowe, D., \& Pearson, M. (2003). A confidential study of deaths after emergency medical admission; issues relating to quality of care. Clinical Medicine, 3(5), 425-434.

Sharp, K. (1998). The case for case studies in nursing research: the problem for generalisation. Journal of Advanced Nursing, 27, 785-789.

Shields, A., Comstock, C., \& Weiss, K. (2004). Variations in asthma care by race/ethnicity among children enrolled in a state Medicaid program. Pediatrics, 113(3), 496-504.

Simons, H. (2009). Case study research in practice. Thousand Oaks: Sage Publications.

Sinclair, A., Prazenica, R., \& Jeffcoat, D. (2008). Optimal and feedback path planning for cooperative attack. Journal of Guidance, Control and Dynamics, 31(6), 1708-1715.

Smith, G. B., \& Poplett, N. (2002). Knowledge of aspects of acute care in trainee doctors. Postgraduate Medical Journal, 78, 335-338. 
Stake, R. (1994). Case Studies. In N. K. Denzin, Lincoln, Y.S (Ed.), Handbook of qualitative research (pp. 236-247). Thousand Oaks: Sage

Stake, R. (1995). The art of case study research. Thousand Oaks: Sage Publications.

Stoeker, R. (1991). Evaluating and rethinking case study. The Sociological Review, $39,88-112$.

Thomson, R. T., Leuttel, D., Healey, F., \& Scobie, S. (2007). Safer care for the acutely ill patient: learning from serious incidents. London: National Patient Safety Agency. Retrieved from http://www.npsa.nhs.uk/nrls/alerts-anddirectives/directives-guidance/acutely-ill-patient/.

Tourangeau, A. E., Doran, D., McGillis Hall, L., O'Brien Pallis, L., Pringle, D., Tu, J., \& Cranley, L.A. (2006). Impact of hospital nursing care on 30 day mortality for acute medical patients. Journal of Advanced Nursing, 57(1), 32-44. doi: 10.1111/j.1365-2648.2006.04084.x

Tuckett, A. G., \& Stewart, D.E. (2004). Collecting qualitative data: Part 11 Journal as a method: experience, rationale and limitations. Contemporary Nurse, 16, 240-251.

United Nations (2009). World population ageing 2009 (Department of Economic and Social Affairs/Population Division). New York. Retrieved from http://www.un.org/esa/population/publications/WPA2009/WPA2009report

Van Weel, C., \& Michels, J. (1997). Dying, not old age, to blame for costs of health care. Lancet, 350 (9085), 1159.

Walker, L. O., \& Avant, K. C. (2005). Strategies for theory construction in nursing (4th ed.). New Jersey: Pearson Prentice Hall.

Weaver, K., Mitcham, C. (2008). Nursing concept analysis in North America: state of the art. Nursing Philosophy, 9, 180-194.

Wheatley, I. (2006). The nursing practice of taking level 1 patient observations. Intensive \& Critical Care Nursing, 22(2), 115-121.

Wilson, J. (1963). Thinking with concepts. Cambridge: Cambridge University Press.

Wood, I., Douglas, J., \& Priest, H. (2004). Education and training for acute care delivery: a needs analysis. Nursing in Critical Care, 9(4), 159-166.

Woods, L., \& Roberts, P. (2003). Requesting access for research purposes: issues and guidelines. Nurse Researcher, 10(3), 38-47. 
World Health Organisation (2003). The health report 2003 - shaping the future.

Geneva. Retrieved from http://www.who.int/whr/2003/en/whr03_en.pdf

Yin, R. (1993). Applications of case study research. California: Sage Publications.

Yin, R. (2003). Case Study Research: Design and methods (3rd ed.). Thousand Oaks: Sage Publications. 